Supporting Information

\title{
4-Methyltrityl-Protected Pyrrole and Imidazole Building Blocks for Solid Phase Synthesis of DNA-Binding Polyamides
}

Benedikt Heinrich and Olalla Vázquez* 


\section{TABLE OF CONTENT}

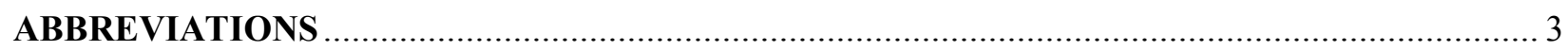

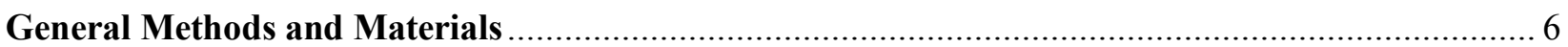

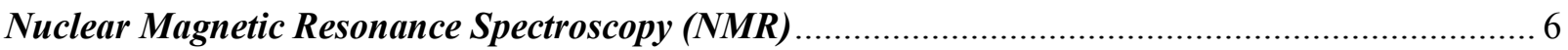

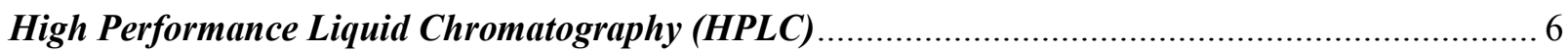

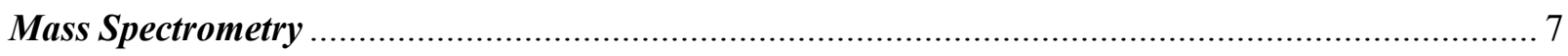

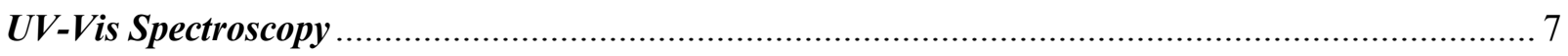

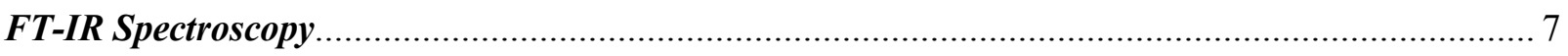

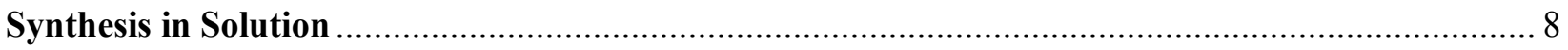

Synthesis of Pyrrole Monomers Nitro-Py-OMe (10), Fmoc-Py-OH (6) and Fmoc-PyIm-OH (8)....... 8

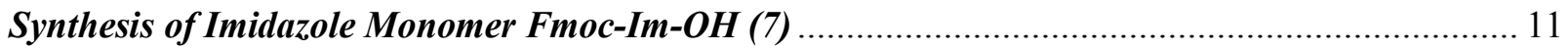

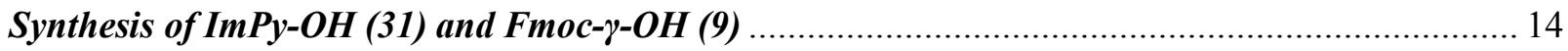

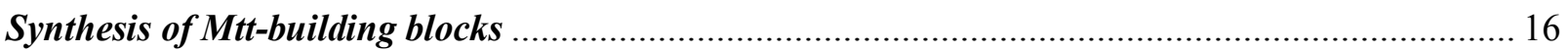

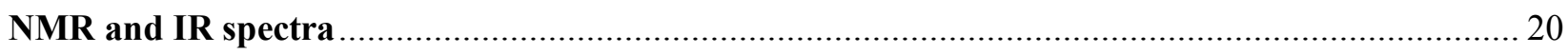

NMR spectra of previously reported compounds .......................................................................... 20

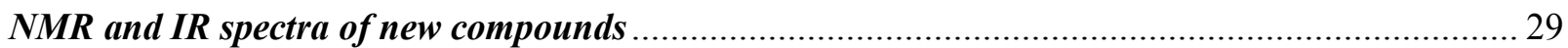

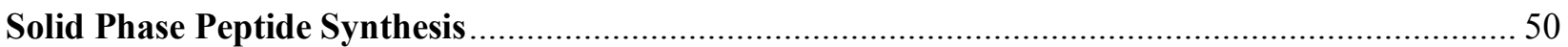

Manual Solid Phase Protocol for Peptide Synthesis ..................................................................... 50

Synthesis and Characterization Data of the Polyamides................................................................ 51

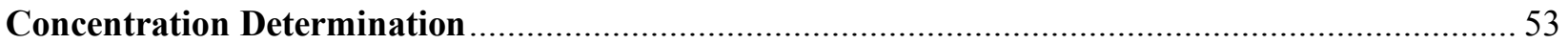

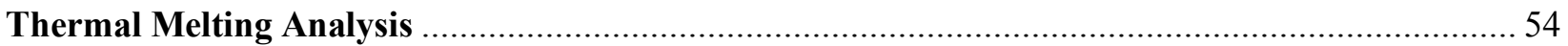

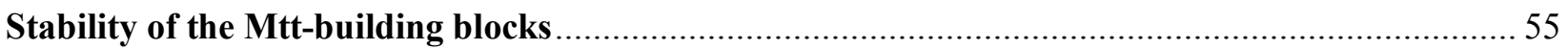

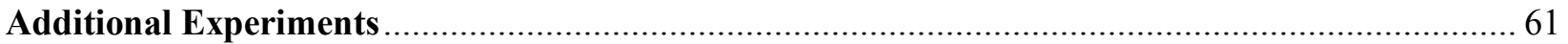

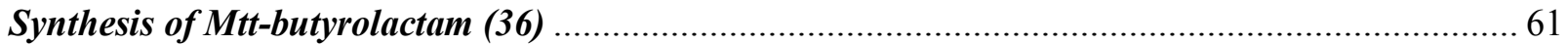

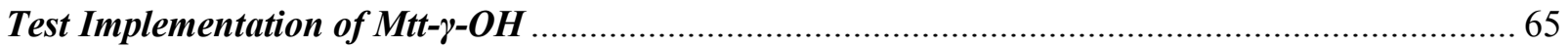

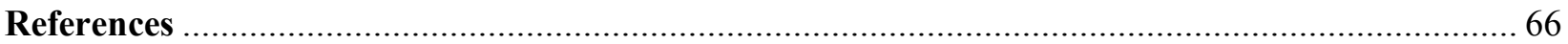




\section{ABBREVIATIONS}

\begin{tabular}{|c|c|}
\hline A & adenine, absorbance \\
\hline $\mathrm{aq}$ & aqueous \\
\hline arom & aromatic \\
\hline $\mathrm{AU}$ & arbitrary units \\
\hline Boc & tert-butyloxycarbonyl \\
\hline br & broad \\
\hline$c$ & concentration \\
\hline $\mathrm{C}$ & cytosine \\
\hline calcd & calculated \\
\hline CI & chemical ionization \\
\hline COSY & correlation spectroscopy \\
\hline $\mathrm{d}$ & doublet \\
\hline DCC & $N, N$ '-dicyclohexylcarbodiimide \\
\hline DIC & $N, N^{\prime}$-diisopropylcarbodiimide \\
\hline DIPEA & $N, N$-diisopropylethylamine \\
\hline DMAP & 4-(dimethylamino)pyridine \\
\hline $\mathrm{Dp}$ & $N, N$-dimethyl-1,3-diaminopropane \\
\hline dsDNA & double-stranded DNA \\
\hline EI & electron ionization \\
\hline ESI & electrospray ionization \\
\hline Fmoc & fluorenylmethyloxycarbonyl \\
\hline FT-IR & fourier-transform infrared spectroscopy \\
\hline G & guanine \\
\hline HATU & hexafluorophosphate azobenzotriazole tetramethyl uronium \\
\hline HMBC & heteronuclear multiple bond correlation spectroscopy \\
\hline $\mathrm{HOBt}$ & 1-hydroxybenzotriazole \\
\hline HPLC & high performance liquid chromatography \\
\hline HRMS & high resolution mass spectrometry \\
\hline
\end{tabular}




\begin{tabular}{|c|c|}
\hline HSQC & heteronuclear single quantum correlation spectroscopy \\
\hline $\operatorname{Im}$ & imidazole \\
\hline$J$ & coupling constant \\
\hline 1 & length \\
\hline $\mathrm{m}$ & multiplet, medium \\
\hline Mtt & 4-methyltrityl \\
\hline NMM & 4-methylmorpholine \\
\hline NMR & nuclear magnetic resonance \\
\hline OSU & $N$-hydroxysuccinimide \\
\hline PHB & 4-hydroxybenzyl \\
\hline $\mathrm{PhSH}$ & thiophenol \\
\hline ppm & parts per million \\
\hline Py & pyrrole \\
\hline PyBOP & (benzotriazol-1-yloxy)tripyrrolidinophosphonium hexafluorophosphate \\
\hline q & quartet \\
\hline $\mathrm{R}_{f}$ & retention factor \\
\hline $\mathrm{RP}$ & reverse-phase \\
\hline $\mathrm{s}$ & singlet, strong \\
\hline SPPS & solid phase peptide synthesis \\
\hline ss & single stranded \\
\hline $\mathrm{t}$ & triplet \\
\hline $\mathrm{T}$ & thymine \\
\hline TG & tentagel \\
\hline$T_{m}$ & melting temperature \\
\hline TMS & trimethylsilyl \\
\hline$t_{R}$ & retention time \\
\hline UV & ultraviolet \\
\hline Vis & visible \\
\hline $\mathrm{w}$ & weak \\
\hline$\beta$ & $\beta$-alanine \\
\hline
\end{tabular}


$\gamma$-aminobutyric acid

chemical shift

$\varepsilon$

extinction coefficient 


\section{General Methods and Materials}

All commercially purchased reagents were used from the following companies without further purification: trichloroacetyl chloride, $N$-methylimidazole, fuming nitric acid, thiophenol, 1-hydroxybenzotriazole hydrate, PyBOP, DMSO for molecular biology and ssDNAs from Sigma Aldrich (USA); sodium hydroxide, potassium hydroxide and concentrated nitric acid from Merck (Germany); DMF peptide grade, HATU, piperidine and NMP from Iris Biotech (Germany); DIPEA, acetic anhydride and Fmoc-Cl from Carl Roth (Germany); $\mathrm{Pd} / \mathrm{C}$, 4-methylmorpholine, 4-aminobutanoic acid and $N, N$-dimethyl-1,3-diaminopropane from Alfa Aesar (USA); pyridine, triethylamine, cesium carbonate, acetonitrile and sodium chloride from VWR (USA); 4-methyltrityl chloride, FmocOSu and (Boc) $)_{2} \mathrm{O}$ from Fluorochem (UK); OxymaPure and DIC from Carbolution (Germany); $N$-methyl pyrrole and benzyl bromide from Acros Organics (Belgium); trifluoroacetic acid from abcr (Germany); dimethylamino pyridine and $N, N$ '-dicyclohexylcarbodiimide from Fluka (USA); sodium bicarbonate from PanReac AppliChem (Germany), reverse phase filter cartridges Oasis HLB 20cc from Waters (Germany) and TG R PHB resin from Rapp Polymere (Germany). Water was purified with a milli-Q Ultra Pure Water Purification System (TKA, Germany). For aqueous solutions for reaction deionized water was used. The buffer for thermal melting analysis was prepared with milli-Q water. For those reactions that require heating, an oil bath was used as heat source.

\section{Nuclear Magnetic Resonance Spectroscopy (NMR)}

All NMR spectra were automatically measured at $300 \mathrm{~K}$ either in a Bruker AV III HD $300 \mathrm{MHz}$ at a frequency of $300 \mathrm{MHz}\left({ }^{1} \mathrm{H}\right)$ or $75 \mathrm{MHz}\left({ }^{13} \mathrm{C}\right)$ or in a Bruker AV III HD $500 \mathrm{MHz}$ at a frequency of $500 \mathrm{MHz}$ $\left({ }^{1} \mathrm{H}\right)$ or $125 \mathrm{MHz}\left({ }^{13} \mathrm{C}\right)$. All chemical shifts $(\delta)$ are given in ppm and are relative to tetramethylsilane (TMS), i.e. $\delta(\mathrm{TMS})=0 \mathrm{ppm}$. As internal standards, deuterated chloroform $\left(\mathrm{CDCl}_{3}\right)$, deuterated methanol $\left(\mathrm{CD}_{3} \mathrm{OD}\right)$, dimethyl sulfoxide (DMSO) with TMS were used. Solvent shifts $(\mathrm{ppm}): \delta\left(\mathrm{CHCl}_{3}\right)=7.26 \mathrm{ppm}\left({ }^{1} \mathrm{H}\right)$, $\delta\left(\mathrm{CHCl}_{3}\right)=77.16 \mathrm{ppm}\left({ }^{13} \mathrm{C}\right), \delta\left(\mathrm{CH}_{3} \mathrm{OH}\right)=3.31 \mathrm{ppm}\left({ }^{1} \mathrm{H}\right), \delta\left(\mathrm{CH}_{3} \mathrm{OH}\right)=49.00 \mathrm{ppm}\left({ }^{13} \mathrm{C}\right), \delta(\mathrm{DMSO})=$ $2.50 \mathrm{ppm}\left({ }^{1} \mathrm{H}\right), \delta(\mathrm{DMSO})=39.52 \mathrm{ppm}\left({ }^{13} \mathrm{C}\right) \cdot{ }^{[\mathrm{i}]}$ The assignment of each signal was based on two-dimensional nuclear magnetic resonance spectroscopy (2D NMR), i.e. heteronuclear single quantum coherence (HSQC) and heteronuclear multiple bond correlation spectroscopy (HMBC). TopSpin was used to analyze the spectra.

\section{High Performance Liquid Chromatography (HPLC)}

For preparative purposes, a PLC 2020 Personal Purification System (Gilson) with a preparative Nucleodur C18 HTec-column ( $5 \mu \mathrm{m}, 250 \times 16 \mathrm{~mm}$; Macherey \& Nagel) using an isocratic regime during the first five minutes, for column equilibration, followed by a linear gradient $5 \%$ to $50 \%$ B in 30 min with a flow rate of $10 \mathrm{~mL} / \mathrm{min}$ at $25^{\circ} \mathrm{C}$ was used. The detection was carried out by measuring the absorption at wavelengths: $220 \mathrm{~nm}$ and $304 \mathrm{~nm}$. Milli-Q water (A) and MeCN (B) were employed as eluents with an addition of $0.1 \%$ of TFA in both solvents.

For analytical purposes, an Agilent 1260 Infinity II HPLC-System (Agilent Technologies) was used with an eclipse XDB-C18 column $(5 \mu \mathrm{m}, 4.6 \times 150 \mathrm{~mm}$, Agilent). The flow rate was $1.0 \mathrm{~mL} / \mathrm{min}$ using an isocratic regime of $5 \% \mathrm{~B}$ during the first five minutes, for column equilibration, followed by the linear in $30 \mathrm{~min}$ at $55^{\circ} \mathrm{C}$. The isocratic regime and the linear gradient are:

1) isocratic: $5 \% \mathrm{~B}$, gradient $5-75 \% \mathrm{~B}$.

2) isocratic: $5 \% \mathrm{~B}$, gradient $5-95 \% \mathrm{~B}$. 
For purity determination, the integrals of the corresponding peaks were set into comparison. Only peaks with a signal intensity $>10 \%$ to the highest peak were taken into consideration.

\section{Mass Spectrometry}

High resolution electrospray ionization mass spectrometry (HR-ESI-MS) was performed on a Finnigan LTQ-FT (Thermo Fischer Scientific). The resolution was set to 100.000. HR-EI-MS and HR-CI-MS was performed on an AccuTOF GCv 4G (JEOL) Time of Flight (TOF) mass spectrometer. An internal or external standard was used for drift time correction.

\section{UV-Vis Spectroscopy}

UV-Vis measurements for concentration determinations were performed on a Tecan $20 \mathrm{M}$ at room temperature using a quarz cuvette (Hellma Analytics; 104-QS) with a pathlength of $1 \mathrm{~cm}$ in a volume of $1 \mathrm{~mL}$.

Thermal melting analysis was performed on a Jasco V-650 UV-Vis spectrophotometer equipped with a Jasco PAC-743 temperature controlled cell holder and a Julabo F250 cooling system. The measurements were performed in a $1 \mathrm{~mL}$ quarz cuvette (Hellma Analytics; 104-QS) with a pathlength of $1 \mathrm{~cm}$, a concentration of $1.0 \mu \mathrm{M}$ of duplex DNA with and without $1.2 \mu \mathrm{M}$ of polyamide in $10 \mathrm{mM} \mathrm{NaH}_{2} \mathrm{PO}_{4} \mathrm{pH}$ 7.4. It was measured at $260 \mathrm{~nm}$, with a medium response, a band width of $20 \mathrm{~nm}$, from $20-95{ }^{\circ} \mathrm{C}$ and a slope of $0.5^{\circ} \mathrm{C} / \mathrm{min}$. Three independent measurements were recorded and the intensity normalized.

\section{FT-IR Spectroscopy}

IR measurements were performed on a Bruker Alpha II Platinum ATR FTIR spectrometer. The absorption bands are given in $\mathrm{cm}^{-1}$, intensities are reported as $\mathrm{s}=$ strong, $\mathrm{m}=$ medium, $\mathrm{w}=$ weak. 


\section{Synthesis in Solution}

\section{Synthesis of Pyrrole Monomers Nitro-Py-OMe (10), Fmoc-Py-OH (6) and Fmoc-PyIm-OH (8)}

This synthesis was reported before by Dervan et al., ${ }^{[\mathrm{ii}]}$ Sugiyama et al. ${ }^{[\mathrm{iii}]}$ Lown et al. ${ }^{[\mathrm{iv}]}$ Pindur et al., ${ }^{[\mathrm{v}]}$ and König et al. ${ }^{[\mathrm{vi}]}$ Such procedure is based in the preparation of the nitro pyrrole methylester 10. ${ }^{[i]}$ We followed the same approach with minor modifications.

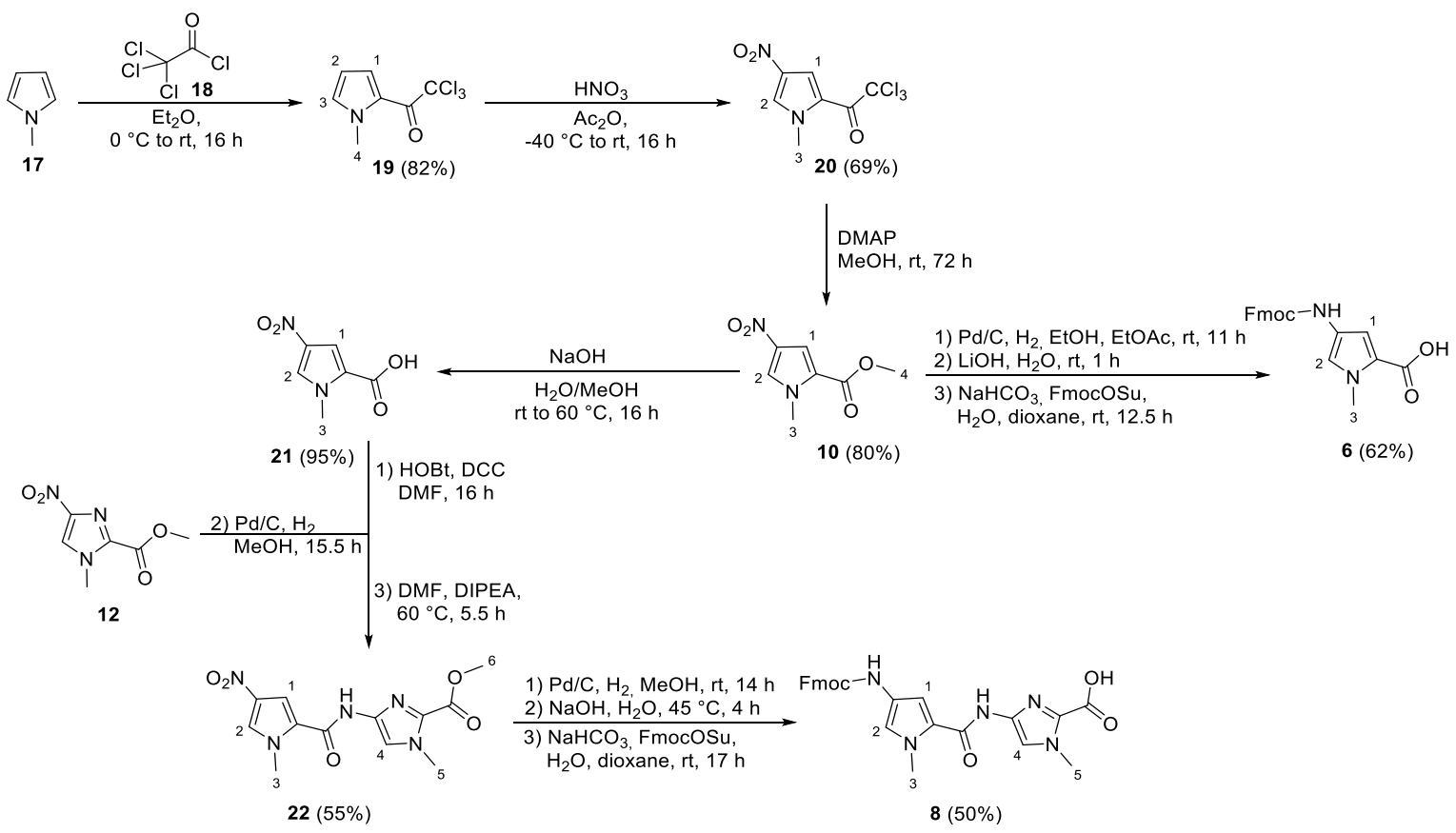

Scheme S1: Synthesis route of nitro-Py-OMe (10) and Fmoc-Py-OH (6) and Fmoc-PyIm-OH (8) starting from $N$-methylpyrrole (17).

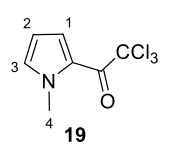

2,2,2-trichloro-1-(1-methyl-1H-pyrrol-2-yl)ethan-1-one (19): Under inert atmosphere, a solution of $\mathrm{N}$ - methylpyrrole $(2.20 \mathrm{~mL}, 25.0 \mathrm{mmol})$ in $\mathrm{Et}_{2} \mathrm{O}(5.0 \mathrm{~mL})$ was added to a solution of trichloroacetyl chloride $(2.80 \mathrm{~mL}, 25.0 \mathrm{mmol})$ in $\mathrm{Et}_{2} \mathrm{O}(5.0 \mathrm{~mL})$ at $0{ }^{\circ} \mathrm{C}$ over $1 \mathrm{~h}$. The reaction mixture was stirred for $16 \mathrm{~h}$ at $\mathrm{rt}$ and neutralized with $\mathrm{K}_{2} \mathrm{CO}_{3}$ solution (aq). It was extracted with $\mathrm{Et}_{2} \mathrm{O}(4 \times 200 \mathrm{~mL})$, dried over $\mathrm{MgSO}_{4}$ and the solvent removed under reduced pressure to yield the desired product $(\mathbf{1 9}, 4.67 \mathrm{~g}, 20.6 \mathrm{mmol}, 82 \%)$ as brown solid. The characterization is in agreement with the literature. ${ }^{\text {[vii] }} \mathbf{R}_{f}=0.60$ (n-pentane/EtOAc 1:1). ${ }^{1} \mathbf{H}-\mathbf{N M R}\left(300 \mathrm{MHz}, \mathrm{DMSO}-d_{6}, \delta\right): 7.46-7.42(\mathrm{~m}$, 2H, CH-1 and $\mathrm{CH}-3), 6.30(\mathrm{dd}, 1 \mathrm{H}, J=4.3 \mathrm{~Hz}, J=2.5 \mathrm{~Hz}, \mathrm{CH}-2), 3.91(\mathrm{~s}, 3 \mathrm{H}, \mathrm{CH}-4) .{ }^{13} \mathbf{C}-\mathbf{N M R}(75 \mathrm{MHz}$, DMSO- $\left.d_{6}, \delta\right): 171.8(\mathrm{C}=\mathrm{O}), 135.3(\mathrm{CH}), 123.6(\mathrm{CH}), 120.7\left(\mathrm{C}_{\mathrm{q}}\right), 109.1(\mathrm{CH}), 96.0\left(\mathrm{CCl}_{3}\right), 36.2\left(\mathrm{CH}_{3}\right)$. HRMS-CI ${ }^{+}(\mathrm{m} / z):[\mathrm{M}+\mathrm{H}]^{+}$calcd for $\mathrm{C}_{7} \mathrm{H}_{6} \mathrm{Cl}_{3} \mathrm{NOH}, 225.95932$; found, 225.95978 .

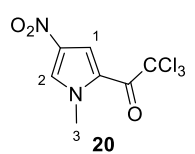

2,2,2-trichloro-1-(1-methyl-4-nitro-1H-pyrrol-2-yl)ethan-1-one (20): Under inert atmosphere, to a solution of 2,2,2-trichloro-1-(1-methyl-1H-pyrrol-2-yl)ethan-1-one (1.50 g, $6.62 \mathrm{mmol})$ in $\mathrm{Ac}_{2} \mathrm{O}(8.00 \mathrm{~mL}, 84.6 \mathrm{mmol})$, conc. $\mathrm{HNO}_{3}(0.60 \mathrm{~mL}, 14.4 \mathrm{mmol})$ was added at $-40{ }^{\circ} \mathrm{C}$ over $30 \mathrm{~min}$. The reaction mixture was slowly warmed up to $\mathrm{rt}$ and stirred for $16 \mathrm{~h}$. 
It was neutralized with $\mathrm{Na}_{2} \mathrm{CO}_{3}$ solution (aq), extracted with EtOAc $(4 \mathrm{x} 150 \mathrm{~mL})$, washed with brine $(3 \mathrm{x}$ $100 \mathrm{~mL}$ ), the organic phases dried over $\mathrm{MgSO}_{4}$ and the solvent removed under reduced pressure to yield the desired product (20, $1.24 \mathrm{~g}, 4.56 \mathrm{mmol}, 69 \%)$ as brown solid. The characterization is in agreement with the literature. ${ }^{[i]]} \mathbf{R}_{\boldsymbol{f}}=0.16$ (n-pentane/EtOAc 10:1). ${ }^{1} \mathbf{H}-\mathbf{N M R}\left(300 \mathrm{MHz}, \mathrm{DMSO}-d_{6}, \delta\right): 8.56(\mathrm{~d}, 1 \mathrm{H}, J=1.6 \mathrm{~Hz}$, $\mathrm{CH}-2), 7.80(\mathrm{~d}, 1 \mathrm{H}, J=1.6 \mathrm{~Hz}, \mathrm{CH}-1), 4.00$ (s, 3H, CH-3). ${ }^{13} \mathbf{C}-\mathbf{N M R}\left(75 \mathrm{MHz}, \mathrm{DMSO}-d_{6}, \delta\right): 172.8(\mathrm{C}=\mathrm{O})$, 134.2 $\left(\mathrm{C}_{\mathrm{q}}\right), 132.5(\mathrm{CH}), 120.6\left(\mathrm{C}_{\mathrm{q}}\right), 116.3(\mathrm{CH}), 94.5\left(\mathrm{CCl}_{3}\right), 50.0\left(\mathrm{CH}_{3}\right)$. HRMS-CI ${ }^{+}(\mathrm{m} / \mathrm{z}):[\mathrm{M}+\mathrm{H}]^{+}$calcd for $\mathrm{C}_{7} \mathrm{H}_{5} \mathrm{Cl}_{3} \mathrm{~N}_{2} \mathrm{O}_{3} \mathrm{H}, 270.94440$; found, 270.94291.

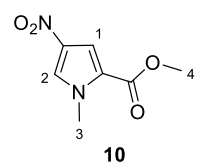

methyl 1-methyl-4-nitro-1H-pyrrole-2-carboxylate (10): To a solution of 2,2,2-trichloro1-(1-methyl-4-nitro-1H-pyrrol-2-yl)ethan-1-one (20, $4.72 \mathrm{~g}, 17.4 \mathrm{mmol})$ in $\mathrm{MeOH}(58 \mathrm{~mL})$, DMAP (212 mg, $1.74 \mathrm{mmol}$ ) was added and the reaction mixture stirred at $\mathrm{rt}$ for $72 \mathrm{~h}$. The solvent was removed and the crude product purified by column chromatography (silica, $n$ pentane/EtOAc $4: 1 \rightarrow 1: 1)$ to yield the desired product $(\mathbf{1 0}, 2.57 \mathrm{~g}, 14.0 \mathrm{mmol}, 80 \%)$ as yellow solid. The characterization is in agreement with the literature. ${ }^{[i]} \mathbf{R}_{f}=0.16$ (n-pentane/EtOAc 10:1). ${ }^{1} \mathbf{H}-\mathbf{N M R}$ $\left(300 \mathrm{MHz}, \mathrm{DMSO}-d_{6}, \delta\right): 8.27(\mathrm{~d}, 1 \mathrm{H}, J=2.0 \mathrm{~Hz}, \mathrm{CH}-2), 7.30$ (d, $\left.1 \mathrm{H}, J=2.0 \mathrm{~Hz}, \mathrm{CH}-1\right), 3.92$ (s, 3H, CH3), $3.80(\mathrm{~s}, 3 \mathrm{H}, \mathrm{CH}-4) .{ }^{13} \mathrm{C}-\mathrm{NMR}\left(75 \mathrm{MHz}, \mathrm{DMSO}-d_{6}, \delta\right): 159.8(\mathrm{C}=\mathrm{O}), 134.2\left(\mathrm{C}_{\mathrm{q}}\right), 129.4(\mathrm{CH}), 122.6\left(\mathrm{C}_{\mathrm{q}}\right)$, $111.5(\mathrm{CH}), 51.7\left(\mathrm{CH}_{3}\right), 37.4\left(\mathrm{CH}_{3}\right)$. HRMS-ESI ${ }^{+}(\mathrm{m} / \mathrm{z})$ : $[\mathrm{M}+\mathrm{H}]^{+}$calcd for $\mathrm{C}_{7} \mathrm{H}_{8} \mathrm{~N}_{2} \mathrm{O}_{4} \mathrm{H}, 185.05623$; found, 185.05665 .

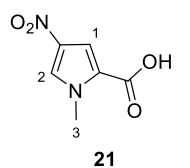

1-methyl-4-nitro-1H-pyrrole-2-carboxylic acid (21): To a solution of methyl 1-methyl-4nitro-1H-pyrrole-2-carboxylate (10, $2.00 \mathrm{~g}, 10.9 \mathrm{mmol})$ in $\mathrm{MeOH}(25.0 \mathrm{~mL}) 1 \mathrm{M} \mathrm{NaOH}(\mathrm{aq}$, $15 \mathrm{~mL}$ ) was added and the reaction mixture stirred at $\mathrm{rt}$ for $16 \mathrm{~h}$ and heated at $60{ }^{\circ} \mathrm{C}$ for $1 \mathrm{~h}$. Afterwards, it was extracted with EtOAc $(3 \times 100 \mathrm{~mL})$ and washed with saturated $\mathrm{NaHCO}_{3}$ (aq, $3 \times 100 \mathrm{~mL})$. The aqueous phases were acidified with $2 \mathrm{M} \mathrm{HCl}(\mathrm{aq})$, extracted with EtOAc $(4 \mathrm{x}$ $200 \mathrm{~mL}$ ), the organic phases dried over $\mathrm{MgSO}_{4}$, filtered and the solvent removed under reduced pressure to yield the desired product $(\mathbf{2 2}, 1.77 \mathrm{~g}, 10.4 \mathrm{mmol}, 95 \%)$ as pale yellow solid. The characterization is in agreement with the literature. ${ }^{\text {viii] }} \mathbf{R}_{f}=0.33$ (EtOAc/formic acid 1:0.02). ${ }^{1} \mathbf{H}-\mathbf{N M R}\left(300 \mathrm{MHz}, \mathrm{DMSO}-d_{6}, \delta\right.$ ): $13.11(\mathrm{~s}, 1 \mathrm{H}, \mathrm{COOH}), 8.21(\mathrm{~d}, 1 \mathrm{H}, J=2.1 \mathrm{~Hz}, \mathrm{CH}-2), 7.25$ (d, $1 \mathrm{H}, J=2.1 \mathrm{~Hz}, \mathrm{CH}-1), 3.91$ (s, 3H, CH-3). ${ }^{13}$ C-NMR (75 MHz, DMSO- $\left.d_{6}, \delta\right): 160.9(\mathrm{C}=\mathrm{O}), 134.0\left(\mathrm{C}_{\mathrm{q}}\right), 129.1(\mathrm{CH}), 123.8\left(\mathrm{C}_{\mathrm{q}}\right), 111.4(\mathrm{CH}), 37.4$ $\left(\mathrm{CH}_{3}\right)$. HRMS-ESI' $(\mathrm{m} / \mathrm{z})$ : [M] ${ }^{-}$calcd for $\mathrm{C}_{6} \mathrm{H}_{5} \mathrm{~N}_{2} \mathrm{O}_{4}, 169.0255$; found, 169.0257 .

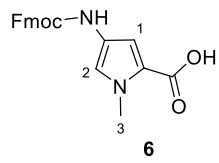

4-((((9H-fluoren-9-yl)methoxy)carbonyl)amino)-1-methyl-1H-pyrrole-2-carboxylic acid (6): Nitro-Py-OMe (10, $2.56 \mathrm{~g}, 13.9 \mathrm{mmol})$ was dissolved in EtOH $(15 \mathrm{~mL})$ and EtOAc $(15 \mathrm{~mL}) . \mathrm{Pd} / \mathrm{C}(10 \%$ dry, $500 \mathrm{mg}, 20 \% \mathrm{w} / \mathrm{w})$ was added and the reaction mixture stirred under hydrogen atmosphere. After $3 \mathrm{~h}$ and $4 \mathrm{~h} \mathrm{Pd} / \mathrm{C}(10 \%$ dry, $500 \mathrm{mg}, 20 \% \mathrm{w} / \mathrm{w})$ was added and the reaction mixture stirred under hydrogen atmosphere. After another $4 \mathrm{~h}$ it was filtered over celite and the solvent removed. The crude was suspended in $2 \mathrm{M} \mathrm{LiOH} \mathrm{(aq,} 35 \mathrm{~mL})$ and stirred at rt for $1 \mathrm{~h}$. It was neutralized with $4 \mathrm{M} \mathrm{HCl}(\mathrm{aq})$, the solvent reduced, the crude dissolved in $\mathrm{H}_{2} \mathrm{O}(15 \mathrm{~mL})$ and 1,4dioxane $(15 \mathrm{~mL}), \mathrm{NaHCO}_{3}(5.86 \mathrm{~g}, 69.6 \mathrm{mmol})$ and $\mathrm{FmocOSu}(5.63 \mathrm{~g}, 16.7 \mathrm{mmol})$ added. The reaction mixture was stirred under nitrogen atmosphere for $12.5 \mathrm{~h}$ at $\mathrm{rt}$. It was diluted with water and acidified with $2 \mathrm{M} \mathrm{HCl}$ (aq). The product was extracted with EtOAc $(4 \times 200 \mathrm{~mL})$, the organic phases dried over $\mathrm{MgSO}_{4}$, filtered and the solvent removed under reduced pressure. It was purified by column chromatography (silica, $\mathrm{CH}_{2} \mathrm{Cl}_{2} / \mathrm{MeOH}$ 100:1 $\rightarrow$ 20:1 $\rightarrow$ 10:1 $\rightarrow$ 10:1 including 1\% formic acid) to yield the desired product (6, $3.12 \mathrm{~g}, 8.61 \mathrm{mmol}, 62 \%)$ as brown solid. The characterization is in agreement with the literature. ${ }^{\text {[vi] }} \mathbf{R}_{f}=$ 
$0.25\left(\mathrm{CH}_{2} \mathrm{Cl}_{2} / \mathrm{MeOH} 20: 1\right) .{ }^{1} \mathrm{H}-\mathrm{NMR}\left(300 \mathrm{MHz}, \mathrm{DMSO}-d_{6}, \delta\right): 12.11$ (s, $\left.1 \mathrm{H}, \mathrm{COOH}\right), 9.41(\mathrm{~s}, 1 \mathrm{H}, \mathrm{N} H)$, 7.90 (d, 2H, $\left.J=7.4 \mathrm{~Hz}, \mathrm{C} H_{\text {arom. }}\right), 7.72$ (d, 2H, $\left.J=7.1 \mathrm{~Hz}, \mathrm{CH}_{\text {arom. }}\right), 7.42$ (d, 2H, $\left.J=7.1 \mathrm{~Hz}, \mathrm{C} H_{\text {arom. }}\right), 7.34$ (d, $2 \mathrm{H}, J=7.4 \mathrm{~Hz}, \mathrm{CH}_{\text {arom }}$ ), 7.03 (s, $\left.1 \mathrm{H}, \mathrm{CH}-2\right), 6.62$ (s, 1H, CH-1), 4.44 (d, 2H, J=6.5 Hz, $\mathrm{CH}_{2}$-Fmoc), 4.28 (t, $1 \mathrm{H}, J=6.5 \mathrm{~Hz}, \mathrm{CH}$-Fmoc), 3.78 (s, 3H, CH$\left.H_{3}-3\right) .{ }^{13} \mathrm{C}-\mathrm{NMR}\left(75 \mathrm{MHz}, \mathrm{DMSO}-d_{6}, \delta\right): 162.0(\mathrm{C}=\mathrm{O})$, $153.3(\mathrm{C}=\mathrm{O}), 143.8\left(2 \mathrm{C}, \mathrm{C}_{\mathrm{q}}\right), 140.8\left(2 \mathrm{C}, \mathrm{C}_{\mathrm{q}}\right), 127.6(2 \mathrm{C}, \mathrm{CH}), 127.1(2 \mathrm{C}, \mathrm{CH}), 125.0(2 \mathrm{C}, \mathrm{CH}), 122.3\left(\mathrm{C}_{\mathrm{q}}\right)$, $120.2\left(\mathrm{C}_{\mathrm{q}}\right), 120.1(2 \mathrm{C}, \mathrm{CH}), 118.6(\mathrm{CH}), 107.5(\mathrm{CH}), 65.4\left(\mathrm{CH}_{2}\right), 46.7(\mathrm{CH}), 36.0\left(\mathrm{CH}_{3}\right)$. HRMS-ESI ${ }^{+}$ $(\mathrm{m} / \mathrm{z}):[\mathrm{M}+\mathrm{Na}]^{+}$calcd for $\mathrm{C}_{21} \mathrm{H}_{18} \mathrm{~N}_{2} \mathrm{O}_{4} \mathrm{Na}, 385.1159$; found, 385.1154 .

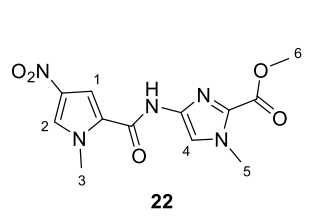

methyl 1-methyl-4-(1-methyl-4-nitro-1H-pyrrole-2-carboxamido)-1Himidazole-2-carboxylate (22): To a solution of nitro-Py-OH (1.04 g, $6.14 \mathrm{mmol})$ in DMF $(10.0 \mathrm{~mL})$ it was added $\mathrm{HOBt} \times \mathrm{H}_{2} \mathrm{O}(1.13 \mathrm{~g}, 7.41 \mathrm{mmol})$ followed by DCC $(1.53 \mathrm{~g}, 7.41 \mathrm{mmol})$. The solution was stirred for $16 \mathrm{~h}$ and the urea side product removed by filtration. Separately, to a solution of nitro-Im-OMe $(1.20 \mathrm{~g}, 6.48 \mathrm{mmol})$ in $\mathrm{MeOH}(11.0 \mathrm{~mL}) \mathrm{Pd} / \mathrm{C}(10 \%$ dry, $240 \mathrm{mg}, 20 \% \mathrm{w} / \mathrm{w})$ was added and the reaction mixture stirred under hydrogen atmosphere for $15.5 \mathrm{~h}$. The catalyst was removed by filtration through celite, the solvent removed and the OBt ester solution directly added. DIPEA $(1.90 \mathrm{~mL}, 10.9 \mathrm{mmol})$ was added and the solution stirred at $60{ }^{\circ} \mathrm{C}$ for $5.5 \mathrm{~h}$. The DMF was removed under reduced pressure and deionized water added to the crude. The formed precipitate was filtered and washed with $1 \mathrm{M} \mathrm{HCl}(\mathrm{aq}, 5 \times 50 \mathrm{~mL})$ and water $(7 \times 50 \mathrm{~mL})$. After freeze drying the precipitate, it was purified by column chromatography (silica, EtOAc/NEt $100: 1$ ) to yield the desired product $(\mathbf{2 2}, 1.04 \mathrm{~g}, 3.38 \mathrm{mmol}, 55 \%)$ as pale brown solid. The characterization is in agreement with the literature. ${ }^{[i x]} \mathbf{R}_{f}=0.21$ ( $n$-pentane/EtOAc 1:1). ${ }^{1} \mathbf{H}$-NMR $\left(300 \mathrm{MHz}, \mathrm{DMSO}-d_{6}, \delta\right): 11.15(\mathrm{~s}, 1 \mathrm{H}$, $\mathrm{NH}), 8.20(\mathrm{~d}, 1 \mathrm{H}, J=2.0 \mathrm{~Hz}, \mathrm{CH}-2), 7.81(\mathrm{~d}, 1 \mathrm{H}, J=2.0 \mathrm{~Hz}, \mathrm{CH}-1), 7.69$ (s, $1 \mathrm{H}, \mathrm{CH}-4), 3.96$ (s, 3H, $\mathrm{CH}_{3}-$ 3), 3.94 (s, 3H, $\left.\mathrm{CH}_{3}-5\right), 3.82$ (s, 3H, $\left.\mathrm{CH}_{3}-6\right) .{ }^{13} \mathbf{C}-\mathbf{N M R}$ (75 MHz, DMSO-d 6 , $\delta$ ): $158.9(\mathrm{C}=\mathrm{O}), 157.3(\mathrm{C}=\mathrm{O})$, 137.1 $\left(\mathrm{C}_{\mathrm{q}}\right), 133.9\left(\mathrm{C}_{\mathrm{q}}\right), 131.0\left(\mathrm{C}_{\mathrm{q}}\right), 128.8(\mathrm{CH}), 125.2\left(\mathrm{C}_{\mathrm{q}}\right), 115.7(\mathrm{CH}), 108.8(\mathrm{CH}), 51.9\left(\mathrm{CH}_{3}\right), 37.8\left(\mathrm{CH}_{3}\right)$, $35.5\left(\mathrm{CH}_{3}\right)$. HRMS-ESI ${ }^{+}(\mathrm{m} / \mathrm{z})$ : $[\mathrm{M}+\mathrm{Na}]^{+}$calcd for $\mathrm{C}_{12} \mathrm{H}_{13} \mathrm{~N}_{5} \mathrm{O}_{5} \mathrm{Na}, 330.0809$; found, 330.0810 .

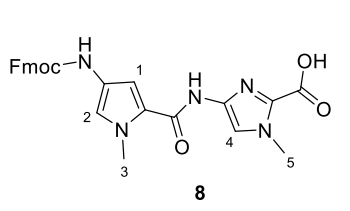

4-(4-(((9H-fluoren-9-yl)methoxy)carbonyl)amino)-1-methyl-1H-pyrrole-2carboxamido)-1-methyl-1H-imidazole-2-carboxylic acid (8): To a solution of nitro-PyIm-OMe (929 mg, $3.02 \mathrm{mmol})$ in $\mathrm{MeOH}(10.0 \mathrm{~mL}), \mathrm{Pd} / \mathrm{C}(10 \%$ dry, $549 \mathrm{mg}, 60 \% \mathrm{w} / \mathrm{w}$ ) was added and the mixture stirred under hydrogen atmosphere for $14 \mathrm{~h}$. The catalyst was removed by filtration through celite, the solvent removed and $1 \mathrm{M} \mathrm{NaOH}$ (aq, $10.0 \mathrm{~mL}$ ) added. After $3 \mathrm{~h}$ at $45^{\circ} \mathrm{C}, 1 \mathrm{M} \mathrm{NaOH}$ (aq, $5.00 \mathrm{~mL}$ ) was added and the solution stirred for another $1 \mathrm{~h} .1 \mathrm{M} \mathrm{HCl}$ (aq) was added until $\mathrm{pH} 7$ was reached. The water was removed under reduced pressure, the residue dissolved in $\mathrm{H}_{2} \mathrm{O}(8.00 \mathrm{~mL})$ and 1,4-dioxane $(5.00 \mathrm{~mL})$ and $\mathrm{NaHCO}_{3}$ $(1.27 \mathrm{~g}, 15.1 \mathrm{mmol})$ and FmocOSu $(1.02 \mathrm{~g}, 3.02 \mathrm{mmol})$ added. The solution was stirred under nitrogen atmosphere at $\mathrm{rt}$ for $16 \mathrm{~h} . \mathrm{H}_{2} \mathrm{O}(5.00 \mathrm{~mL})$ and 1,4-dioxane $(5.00 \mathrm{~mL})$ were added and the solution stirred for another $40 \mathrm{~min}$. The dioxane was removed under reduced pressure and the formed precipitate filtered and washed with water $(6 \times 50 \mathrm{~mL})$. The residue was freeze dried and purified by column chromatography

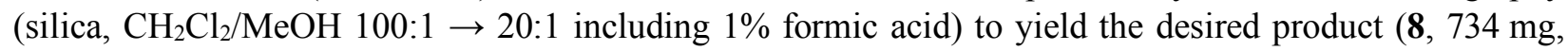
$1.51 \mathrm{mmol}, 50 \%$ ) as pale brown solid. The characterization is in agreement with the literature. ${ }^{[i i i]} \mathbf{R}_{f}=0.33$ (EtOAc). ${ }^{1} \mathrm{H}-\mathrm{NMR}\left(300 \mathrm{MHz}, \mathrm{DMSO}-d_{6}, \delta\right): 10.57(\mathrm{~s}, 1 \mathrm{H}, \mathrm{COOH}), 9.46(\mathrm{~s}, 1 \mathrm{H}, \mathrm{N} H), 8.15(\mathrm{~s}, 1 \mathrm{H}, \mathrm{N} H)$, 7.91 (d, 2H, J=7.3 Hz, CH $\left.H_{\text {arom. }}\right), 7.74$ (d, 2H, J=6.5 Hz, CH $\left.H_{\text {arom. }}\right), 7.57$ (s, 1H, CH-4), $7.47-7.31$ (m, 4H, $\mathrm{CH}_{\text {arom. }}$ ), 7.01 (s, 1H, CH-2), 6.95 (s, 1H, CH-1), 4.47 - 4.37 (m, 2H, $\mathrm{CH}_{2}$-Fmoc.), 4.33 - 4.24 (m, 1H, $\mathrm{CH}-$ Fmoc), 3.91 (s, 3H, $\mathrm{CH}_{3}-3$ ), 3.82 (s, 3H, $\left.\mathrm{CH}_{3}-5\right) .{ }^{13} \mathbf{C}-\mathrm{NMR}\left(125 \mathrm{MHz}, \mathrm{DMSO}-d_{6}, \delta\right): 172.8(\mathrm{C}=\mathrm{O}), 163.2$ $(\mathrm{C}=\mathrm{O}), 153.5(\mathrm{C}=\mathrm{O}), 143.9\left(2 \mathrm{C}, \mathrm{C}_{\mathrm{q}}\right), 140.8\left(2 \mathrm{C}, \mathrm{C}_{\mathrm{q}}\right), 137.2\left(\mathrm{C}_{\mathrm{q}}\right), 132.6\left(\mathrm{C}_{\mathrm{q}}\right), 127.7(2 \mathrm{C}, \mathrm{CH}), 127.1(2 \mathrm{C}$, $\mathrm{CH}), 125.2(2 \mathrm{C}, \mathrm{CH}), 122.6\left(\mathrm{C}_{\mathrm{q}}\right), 122.1\left(\mathrm{C}_{\mathrm{q}}\right), 120.2(2 \mathrm{C}, \mathrm{CH}), 118.1(\mathrm{CH}), 114.6(\mathrm{CH}), 105.0(\mathrm{CH}), 65.5$ 
$\left(\mathrm{CH}_{2}\right), 46.7(\mathrm{CH}), 36.3\left(\mathrm{CH}_{3}\right), 35.4\left(\mathrm{CH}_{3}\right)$. HRMS-ESI ${ }^{+}(\mathrm{m} / \mathrm{z})$ : $[\mathrm{M}+\mathrm{H}]^{+}$calcd for $\mathrm{C}_{26} \mathrm{H}_{23} \mathrm{~N}_{5} \mathrm{O} 5 \mathrm{H}, 486.1777$; found, 486.1772 .

\section{Synthesis of Imidazole Monomer Fmoc-Im-OH (7)}

This synthesis was reported before by Dervan et al., ${ }^{[i i x]}$ and Shibuya et al., ${ }^{[\mathrm{xi}]}$ Such procedure is based in the preparation of the nitro imidazole methylester $\mathbf{1 2}$. We followed the same approach with minor modifications.

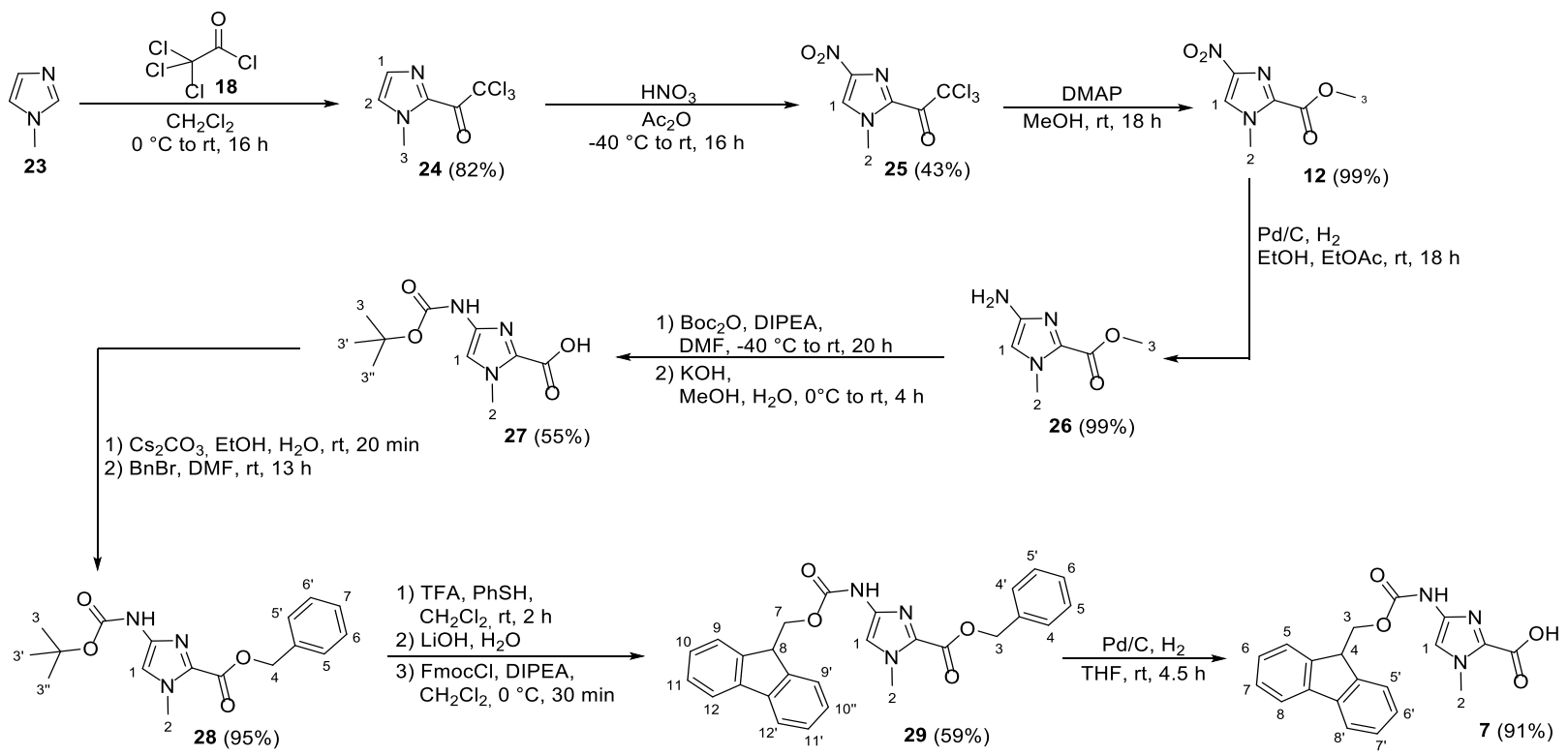

Scheme S2: Synthesis route of nitro-Im-OMe (12) and Fmoc-Im-OH (7) starting from $N$-methylimidazole (23).

2,2,2-trichloro-1-(1-methyl-1H-imidazol-2-yl)ethan-1-one (24): Under inert atmosphere, a

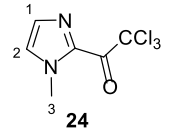
solution of $\mathrm{N}$-methylimidazole $(5.58 \mathrm{~mL}, 70.0 \mathrm{mmol})$ in $\mathrm{CH}_{2} \mathrm{Cl}_{2}(30 \mathrm{~mL})$ was added to a solution of trichloroacetyl chlorid $(7.88 \mathrm{~mL}, 70.2 \mathrm{mmol})$ in $\mathrm{CH}_{2} \mathrm{Cl}_{2}(50 \mathrm{~mL})$ at $0{ }^{\circ} \mathrm{C}$ over $1.5 \mathrm{~h}$. The reaction mixture was stirred for $16 \mathrm{~h}$ at $\mathrm{rt}, \mathrm{CH}_{2} \mathrm{Cl}_{2}(40 \mathrm{~mL})$ and triethylamine $(9.75 \mathrm{~mL}$, $70.2 \mathrm{mmol}$ ) were added at $0{ }^{\circ} \mathrm{C}$ over $30 \mathrm{~min}$. The crude was purified by flash column chromatography (silica, $n$-pentane/EtOAc 1:1) to yield the desired product $(\mathbf{2 4}, 13.0 \mathrm{~g}, 57.2 \mathrm{mmol}, 82 \%)$ as yellow solid. The characterization is in agreement with the literature. ${ }^{[x i i]} \mathbf{R}_{f}=0.72$ (n-pentane/EtOAc 1:1). ${ }^{1} \mathbf{H}-\mathbf{N M R}$ (300 MHz, $\left.\mathrm{CD}_{3} \mathrm{OD}, \delta\right): 7.31$ (s, $\left.1 \mathrm{H}, \mathrm{CH}-2\right), 7.08$ (s, $\left.1 \mathrm{H}, \mathrm{CH}-1\right), 4.01$ (s, 3H, $\left.\mathrm{CH}_{3}-3\right) .{ }^{13} \mathbf{C}-\mathbf{N M R}(75 \mathrm{MHz}$, $\left.\mathrm{CD}_{3} \mathrm{OD}, \delta\right): 160.3(\mathrm{C}=\mathrm{O}), 137.8\left(\mathrm{C}_{\mathrm{q}}\right), 129.4(\mathrm{CH}), 128.1(\mathrm{CH}), 119.5\left(\mathrm{CCl}_{3}\right), 36.2\left(\mathrm{CH}_{3}\right) . \mathbf{H R M S}-\mathbf{C I}^{+}(\mathrm{m} / \mathrm{z})$ : $[\mathrm{M}+\mathrm{H}]^{+}$calcd for $\mathrm{C}_{6} \mathrm{H}_{5} \mathrm{Cl}_{3} \mathrm{~N}_{2} \mathrm{O}_{1} \mathrm{H}, 226.95457$; found, 226.95069 .

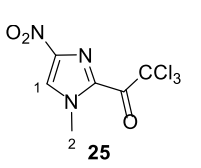

2,2,2-trichloro-1-(1-methyl-4-nitro-1H-imidazol-2-yl)ethan-1-one (25): Under inert atmosphere, $\mathrm{Im}_{-\mathrm{CCl}}(\mathbf{2 4}, 12.7 \mathrm{~g}, 56.0 \mathrm{mmol})$ was dissolved in $\mathrm{Ac}_{2} \mathrm{O}(72.6 \mathrm{~mL}, 768 \mathrm{mmol})$ and cooled to $-40{ }^{\circ} \mathrm{C}$. Fuming $\mathrm{HNO}_{3}(15.2 \mathrm{~mL}, 364 \mathrm{mmol})$ was added carefully dropwise over $1.5 \mathrm{~h}$. The reaction mixture was slowly warmed it up to $\mathrm{rt}$ and stirred with the flask placed in a water bath for $16 \mathrm{~h}$. Afterwards the reaction was neutralized with saturated $\mathrm{Na}_{2} \mathrm{CO}_{3}(\mathrm{aq})$, at $0{ }^{\circ} \mathrm{C}$, extracted with $\mathrm{CH}_{2} \mathrm{Cl}_{2}(4 \times 200 \mathrm{~mL})$ and dried over $\mathrm{MgSO}_{4}$. The solvent was removed to yield the desired product $(\mathbf{2 5}, 6.52 \mathrm{~g}, 23.9 \mathrm{mmol}, 43 \%)$ as pale brown solid. The characterization is in agreement with the literature. ${ }^{[\mathrm{xii}]} \mathbf{R}_{f}=0.55$ (n-pentane/EtOAc 1:1). ${ }^{1} \mathbf{H}-\mathbf{N M R}\left(300 \mathrm{MHz}, \mathrm{CDCl}_{3}, \delta\right.$ ): 7.95 (s, $1 \mathrm{H}, \mathrm{CH}-1$ ), 4.16 (s, 
3H, $\left.\mathrm{CH}_{3}-2\right) .{ }^{13} \mathbf{C}-\mathbf{N M R}\left(75 \mathrm{MHz}, \mathrm{CDCl}_{3}, \delta\right): 173.0(\mathrm{C}=\mathrm{O}), 146.0\left(\mathrm{C}_{\mathrm{q}}\right), 133.8\left(\mathrm{C}_{\mathrm{q}}\right), 126.1(\mathrm{CH}), 93.7\left(\mathrm{CCl}_{3}\right)$, $38.4\left(\mathrm{CH}_{3}\right)$. HRMS-CI ${ }^{+}(\mathrm{m} / \mathrm{z})$ : $[\mathrm{M}+\mathrm{H}]^{+}$calcd for $\mathrm{C}_{6} \mathrm{H}_{4} \mathrm{Cl}_{3} \mathrm{~N}_{3} \mathrm{O}_{3} \mathrm{H}, 271.93965$; found, 271.94021 .

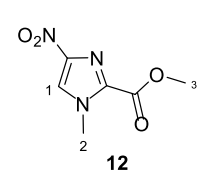

methyl 1-methyl-4-nitro-1H-imidazole-2-carboxylate (12): $\quad \mathrm{Nitro}-\mathrm{Im}_{-} \mathrm{CCl}_{3} \quad(7.17 \mathrm{~g}$, $26.3 \mathrm{mmol}$ ) was suspended in MeOH $(145 \mathrm{~mL})$, followed by DMAP (437 mg, $3.58 \mathrm{mmol})$ addition. The resulting reaction mixture stirred for $18 \mathrm{~h}$ at $\mathrm{rt}$. The crude was purified by flash column chromatography (silica, $n$-pentane/EtOAc: $4: 1 \rightarrow 1: 1 \rightarrow 1: 2$ ) to yield the desired product $(\mathbf{1 2}, 4.87 \mathrm{~g}, 26.3 \mathrm{mmol},>99 \%)$ as pale yellow solid. The characterization is in agreement with the literature. ${ }^{[x i i]} \mathbf{R}_{f}=0.33$ (n-pentane/EtOAc 1:1). ${ }^{1} \mathbf{H}-\mathbf{N M R}\left(300 \mathrm{MHz}, \mathrm{CD}_{3} \mathrm{OD}, \delta\right.$ ): 8.29 (s, 1H, CH-1), 4.08 (s, 3H, $\left.\mathrm{CH}_{3}-2\right), 3.96\left(\mathrm{~s}, 3 \mathrm{H}, \mathrm{CH}_{3}-3\right) .{ }^{13} \mathbf{C}-\mathbf{N M R}\left(75 \mathrm{MHz}, \mathrm{CD}_{3} \mathrm{OD}, \delta\right): 159.6(\mathrm{C}=\mathrm{O}), 142.9\left(\mathrm{C}_{\mathrm{q}}\right), 136.6\left(\mathrm{C}_{\mathrm{q}}\right)$, $126.9(\mathrm{CH}), 53.2\left(\mathrm{CH}_{3}\right), 37.5\left(\mathrm{CH}_{3}\right)$. HRMS-ESI ${ }^{+}(\mathrm{m} / \mathrm{z})$ : $[\mathrm{M}+\mathrm{Na}]^{+}$calcd for $\mathrm{C}_{6} \mathrm{H}_{7} \mathrm{~N}_{3} \mathrm{O}_{4} \mathrm{Na}, 208.0329$; found, 208.0328.

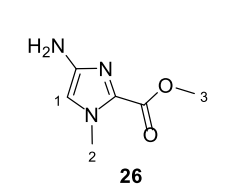

methyl 4-amino-1-methyl-1H-imidazole-2-carboxylate (26): Nitro-Im-OMe (4.83 g, $26.1 \mathrm{mmol})$ was suspended in $\mathrm{EtOH}(75 \mathrm{~mL})$. EtOAc $(75 \mathrm{~mL})$ and $\mathrm{Pd} / \mathrm{C} 10 \%, 50 \%$ wet $(0.1 \mathrm{w} / \mathrm{w} \%)$ in a slurry of EtOAc $(15 \mathrm{~mL})$ was added and the mixture stirred in the dark under hydrogen atmosphere for $18 \mathrm{~h}$. The crude was filtered over celite and the solvent removed under reduced pressure to yield the desired product $(\mathbf{2 6}, 4.01 \mathrm{~g}, 25.8 \mathrm{mmol}, 99 \%)$ as pale yellow solid. The characterization is in agreement with the literature. ${ }^{[\mathrm{x}]} \mathbf{R}_{f}=0.07$ (EtOAc). ${ }^{1} \mathbf{H}-\mathbf{N M R}$ (300 MHz, $\left.\mathrm{CD}_{3} \mathrm{OD}, \delta\right): 6.55$ (s, $\left.1 \mathrm{H}, \mathrm{CH}-1\right), 4.83$ (s, 2H, NH ), 3.90 (s, 3H, $\left.\mathrm{CH}_{3}-2\right), 3.86$ (s, 3H, $\left.\mathrm{CH}_{3}-3\right)$. ${ }^{13}$ C-NMR $\left(75 \mathrm{MHz}, \mathrm{CD}_{3} \mathrm{OD}, \delta\right): 160.1(\mathrm{C}=\mathrm{O}), 147.3\left(\mathrm{C}_{\mathrm{q}}\right), 132.2\left(\mathrm{C}_{\mathrm{q}}\right), 111.0(\mathrm{CH}), 52.2\left(\mathrm{CH}_{3}\right), 36.0\left(\mathrm{CH}_{3}\right)$. HRMS-ESI ${ }^{+}(\mathrm{m} / \mathrm{z}):[\mathrm{M}+\mathrm{Na}]^{+}$calcd for $\mathrm{C}_{6} \mathrm{H}_{9} \mathrm{~N}_{3} \mathrm{O}_{2} \mathrm{Na}, 178.0587$; found, 178.0588.

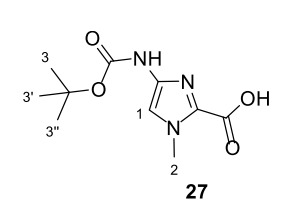

4-((tert-butoxycarbonyl)amino)-1-methyl-1H-imidazole-2-carboxylic acid (27): $\mathrm{NH}_{2}$-Im-OMe (3.97 g, $\left.25.6 \mathrm{mmol}\right)$ was dissolved in DMF $(30 \mathrm{~mL})$. Afterwards DIPEA $(7.52 \mathrm{~mL}, 44.3 \mathrm{mmol})$ and $\mathrm{Boc}_{2} \mathrm{O}(10.9 \mathrm{~g}, 50.0 \mathrm{mmol})$ were added. The mixture was stirred in the dark for $18 \mathrm{~h}$ at rt and heated at $40{ }^{\circ} \mathrm{C}$ for other $2 \mathrm{~h}$. The reaction mixture was extracted with $\mathrm{Et}_{2} \mathrm{O}(4 \times 200 \mathrm{~mL})$ and washed with brine (aq, $\left.3 \times 100 \mathrm{~mL}\right), 10 \%$ citric acid (aq, $3 \times 100 \mathrm{~mL}$ ), brine (aq, $3 \times 100 \mathrm{~mL}$ ), saturated $\mathrm{NaHCO}_{3}$ (aq, $3 \times 100 \mathrm{~mL}$ ) and brine (aq, $3 \times$ $100 \mathrm{~mL}$ ). The organic phases were dried over $\mathrm{MgSO}_{4}$ and the solvent removed under reduced pressure. The crude was dissolved in $\mathrm{MeOH}(80 \mathrm{~mL})$ and $1 \mathrm{M} \mathrm{KOH}(\mathrm{aq}, 50 \mathrm{~mL})$ was added at $0{ }^{\circ} \mathrm{C}$ and stirred in the dark for $30 \mathrm{~min}$ and another $3.5 \mathrm{~h}$ at rt. The reaction mixture was extracted with $\mathrm{Et}_{2} \mathrm{O}(3 \times 150 \mathrm{~mL})$, the aqueous phase cooled to $0{ }^{\circ} \mathrm{C}$ and $10 \% \mathrm{NaHSO}_{4}$ (aq) added until $\mathrm{pH}$ 3. The mixture was extracted with EtOAc $(4 \mathrm{x}$ $200 \mathrm{~mL}$ ), the organic phases dried over $\mathrm{MgSO}_{4}$ and the solvent removed under reduced pressure to yield the desired product $(27,3.38 \mathrm{~g}, 14.0 \mathrm{mmol}, \sim 55 \%)$ as pale brown solid. The characterization is in agreement with the literature. ${ }^{[\mathrm{xii}]} \mathbf{R}_{\boldsymbol{f}}=0.02$ (n-pentane/EtOAc 1:1). ${ }^{1} \mathbf{H}-\mathrm{NMR}\left(300 \mathrm{MHz}, \mathrm{CD}_{3} \mathrm{OD}, \delta\right): 7.20$ (s, $1 \mathrm{H}, \mathrm{CH}$ 1), 4.00 (s, 3H, $\left.\mathrm{CH}_{3}-2\right), 1.51$ (s, 9H, $\left.\mathrm{CH}_{3}-3\right) .{ }^{13} \mathbf{C}-\mathbf{N M R}\left(75 \mathrm{MHz}, \mathrm{CD}_{3} \mathrm{OD}, \delta\right): 160.9(\mathrm{C}=\mathrm{O}), 152.9(\mathrm{C}=\mathrm{O})$, $137.5\left(\mathrm{C}_{\mathrm{q}}\right), 134.5\left(\mathrm{C}_{\mathrm{q}}\right), 112.4(\mathrm{CH}), 79.5\left(\mathrm{C}_{\mathrm{q}}\right), 35.7\left(\mathrm{CH}_{3}\right), 28.6\left(3 \mathrm{C}, \mathrm{CH}_{3}\right)$. HRMS-ESI $^{+}(\mathrm{m} / \mathrm{z}):[\mathrm{M}+\mathrm{Na}]^{+}$ calcd for $\mathrm{C}_{10} \mathrm{H}_{15} \mathrm{~N}_{3} \mathrm{O}_{4} \mathrm{Na}$, 264.0955; found, 264.0955. 


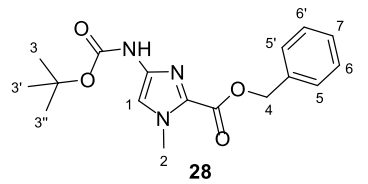

benzyl 4-((tert-butoxycarbonyl)amino)-1-methyl-1H-imidazole-2-carboxylate (28): Boc-Im-OH (3.34 g, $10.1 \mathrm{mmol})$ was suspended in 66\% EtOH $(67 \mathrm{~mL})$ and $\mathrm{Cs}_{2} \mathrm{CO}_{3}$ (3.34 g, $10.3 \mathrm{mmol}$ ) added. After stirring for $20 \mathrm{~min}$ at $\mathrm{rt}$, the suspension was filtered over glass wool and the solvent removed under reduced pressure. The crude was dissolved in DMF (180 mL), BnBr $(1.75 \mathrm{~mL}, 14.6 \mathrm{mmol})$ added and the mixture stirred for $13 \mathrm{~h}$. Deionized water was added. It was extracted with EtOAc $(4 \times 200 \mathrm{~mL})$, washed with water $(3 \times 100 \mathrm{~mL})$ and the organic phases dried over $\mathrm{MgSO}_{4}$. The crude was purified by flash column chromatography (silica, $n$-pentane/EtOAc: $10: 1 \rightarrow 5: 1 \rightarrow 1: 1)$ to yield the desired product $(\mathbf{2 8}, 3.17 \mathrm{~g}, 9.57 \mathrm{mmol}, 95 \%)$ as pale brown solid. The characterization is in agreement with the literature ${ }^{[x i i]} \mathbf{R}_{f}=0.65$ ( $n$-pentane/EtOAc 1:1). ${ }^{1}$ H-NMR $\left(300 \mathrm{MHz}, \mathrm{CD}_{3} \mathrm{OD}, \delta\right): 7.49-7.42$ (m, 2H, CH arom. $\left._{\text {. }}\right)$ 7.40-7.32 (m, 3H, CHarom.), 7.25 (s, $1 \mathrm{H}, \mathrm{CH}-$ 1), 5.35 (s, 2H, $\left.\mathrm{CH}_{2}-4\right), 3.96$ (s, 3H, $\left.\mathrm{CH}_{3}-2\right), 1.50$ (s, 9H, CH$\left.H_{3}-3\right) .{ }^{13} \mathbf{C}-\mathbf{N M R}\left(75 \mathrm{MHz}, \mathrm{CD}_{3} \mathrm{OD}, \delta\right): 159.5$ $(\mathrm{C}=\mathrm{O}), 154.8(\mathrm{C}=\mathrm{O}), 139.3\left(\mathrm{C}_{\mathrm{q}}\right), 137.2\left(\mathrm{C}_{\mathrm{q}}\right), 135.5\left(\mathrm{C}_{\mathrm{q}}\right), 129.6(2 \mathrm{C}, \mathrm{CH}), 129.5(2 \mathrm{C}, \mathrm{CH}), 129.4(\mathrm{CH}), 115.6$ $(\mathrm{CH}), 81.3\left(\mathrm{C}_{\mathrm{q}}\right), 67.9\left(\mathrm{CH}_{2}\right), 36.5\left(\mathrm{CH}_{3}\right), 28.6\left(3 \mathrm{C}, \mathrm{CH}_{3}\right)$. HRMS-ESI ${ }^{+}(\mathrm{m} / \mathrm{z})$ : $[\mathrm{M}+\mathrm{Na}]^{+}$calcd for $\mathrm{C}_{17} \mathrm{H}_{21} \mathrm{~N}_{3} \mathrm{O}_{4} \mathrm{Na}$, 354.1424; found, 354.1425.

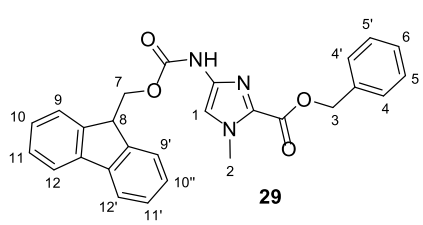

benzyl 4-(((9H-fluoren-9-yl)methoxy)carbonyl)amino)-1-methyl-1Himidazole-2-carboxylate (29): Boc-Im-OBn (3.13 g, $9.45 \mathrm{mmol})$ was dissolved in $\mathrm{CH}_{2} \mathrm{Cl}_{2}(12.5 \mathrm{~mL})$ and a mixture of TFA $(8.2 \mathrm{~mL}), \mathrm{CH}_{2} \mathrm{Cl}_{2}$ $(4.4 \mathrm{~mL})$ and $\mathrm{PhSH}(960 \mu \mathrm{L})$ was added. After stirring for $2 \mathrm{~h}$ in the dark, the reaction mixture was added dropwise at $0{ }^{\circ} \mathrm{C}$ to $\left.2 \mathrm{M} \mathrm{LiOH} \mathrm{(aq,} 100 \mathrm{~mL}\right)$. It was extracted with $\mathrm{Et}_{2} \mathrm{O}(4 \times 100 \mathrm{~mL})$, washed with $\left.2 \mathrm{M} \mathrm{LiOH} \mathrm{(aq,} 3 \times 80 \mathrm{~mL}\right)$ and the organic phases dried over $\mathrm{MgSO}_{4}$. The solvent was removed under reduced pressure. The crude, under inert atmosphere, was dissolved in $\mathrm{CH}_{2} \mathrm{Cl}_{2}(20 \mathrm{~mL})$ and DIPEA $(6.4 \mathrm{~mL}, 36.8 \mathrm{mmol})$ and Fmoc-Cl $(2.46 \mathrm{~g}, 9.52 \mathrm{mmol})$ were added at $0{ }^{\circ} \mathrm{C}$. It was stirred in the dark for $30 \mathrm{~min}$ at $0{ }^{\circ} \mathrm{C}$. It was acidified with $1 \mathrm{M} \mathrm{HCl}$ (aq), extracted with $\mathrm{CH}_{2} \mathrm{Cl}_{2}(4 \times 150 \mathrm{~mL})$, the organic phases dried over $\mathrm{MgSO}_{4}$ and the solvent removed under reduced pressure. The crude was purified by flash column chromatography (silica, $n$-pentane/EtOAc: 10:1 $\rightarrow 4: 1 \rightarrow$ $0: 1)$ to yield the desired product $(\mathbf{2 9}, 2.52 \mathrm{~g}, 5.56 \mathrm{mmol}, 59 \%)$ as a colorless solid. The characterization is in agreement with the literature. ${ }^{[\mathrm{x}]} \mathbf{R}_{\boldsymbol{f}}=0.73$ ( $n$-pentane/EtOAc 1:1). ${ }^{1} \mathbf{H}-\mathbf{N M R}\left(300 \mathrm{MHz}, \mathrm{DMSO}-d_{6}, \delta\right)$ : 10.36 (s, $1 \mathrm{H}, \mathrm{NH}), 7.90$ (d, 2H, $\left.J=7.5 \mathrm{~Hz}, \mathrm{C} H_{\text {arom. }}\right), 7.76$ (d, 2H, $\left.J=7.0 \mathrm{~Hz}, \mathrm{CH}_{\text {arom. }}\right), 7.47-7.30$ (m, $10 \mathrm{H}$, $\mathrm{CH}_{\text {arom- }}$ and $\left.\mathrm{CH}-1\right), 5.30\left(\mathrm{~s}, 2 \mathrm{H}, \mathrm{CH}_{2}-3\right), 4.36-4.23$ (m, $3 \mathrm{H}, \mathrm{CH}_{2}-7$ and $\left.\mathrm{CH}-8\right), 3.92\left(\mathrm{~s}, 3 \mathrm{H}, \mathrm{CH}_{3}-2\right) .{ }^{13} \mathbf{C}-$ NMR (75 MHz, DMSO- $\left.d_{6}, \delta\right): 158.1(\mathrm{C}=\mathrm{O}), 153.2(\mathrm{C}=\mathrm{O}), 143.7\left(2 \mathrm{C}, \mathrm{C}_{\mathrm{q}}\right), 140.7\left(2 \mathrm{C}, \mathrm{C}_{\mathrm{q}}\right), 137.9\left(\mathrm{C}_{\mathrm{q}}\right), 135.7$ $\left(\mathrm{C}_{\mathrm{q}}\right), 130.8\left(\mathrm{C}_{\mathrm{q}}\right), 128.4(2 \mathrm{C}, \mathrm{CH}), 128.3(2 \mathrm{C}, \mathrm{CH}), 128.2(\mathrm{CH}), 127.6(2 \mathrm{C}, \mathrm{CH}), 127.0(2 \mathrm{C}, \mathrm{CH}), 125.3(2 \mathrm{C}$, $\mathrm{CH}), 120.0(2 \mathrm{C}, \mathrm{CH}), 113.9(\mathrm{CH}), 66.1\left(\mathrm{CH}_{2}\right), 66.0\left(\mathrm{CH}_{2}\right), 46.4(\mathrm{CH}), 35.4\left(\mathrm{CH}_{3}\right)$. HRMS-ESI $^{+}(\mathrm{m} / \mathrm{z})$ : $[\mathrm{M}+\mathrm{Na}]^{+}$calcd for $\mathrm{C}_{27} \mathrm{H}_{23} \mathrm{~N}_{3} \mathrm{O}_{4} \mathrm{Na}, 476.1581$; found, 476.1582 .

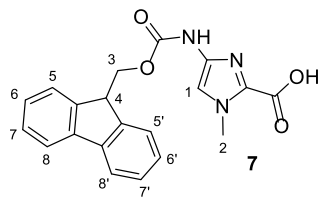

4-(((9H-fluoren-9-yl)methoxy)carbonyl)amino)-1-methyl-1H-imidazole-2carboxylic acid (7): Fmoc-Im-OBn (100 mg, $0.22 \mathrm{mmol})$ was dissolved in THF $(2.0 \mathrm{~mL})$ and $\mathrm{Pd} / \mathrm{C} 10 \%$ dry $(0.25 w / w \%)$ was added. The reaction mixture was stirred under hydrogen atmosphere for $4.5 \mathrm{~h}$ at $\mathrm{rt}$. The reaction mixture was filtered over celite and the solvent removed under reduced pressure to yield the desired product $(7,72.7 \mathrm{mg}, 0.20 \mathrm{mmol}, 91 \%)$ as a pale grey solid. The characterization is in agreement with the literature. ${ }^{[\mathrm{xiv}]} \mathbf{R}_{\boldsymbol{f}}=0.32\left(\mathrm{CH}_{2} \mathrm{Cl}_{2} / \mathrm{MeOH} /\right.$ formic acid 100:10:1). ${ }^{1} \mathbf{H}-\mathbf{N M R}\left(500 \mathrm{MHz}, \mathrm{DMSO}-d_{6}, \delta\right): 11.71$ (s, $1 \mathrm{H}, \mathrm{COOH}), 10.30(\mathrm{~s}, 1 \mathrm{H}, \mathrm{NH}), 7.91$ (d, 2H, $\left.J=7.4 \mathrm{~Hz}, \mathrm{CH}_{\text {arom. }}\right), 7.77$ (d, $\left.2 \mathrm{H}, J=7.4 \mathrm{~Hz}, \mathrm{C} H_{\text {arom. }}\right), 7.43$ (dd, $\left.2 \mathrm{H}, J=7.4 \mathrm{~Hz}, J=7.4 \mathrm{~Hz}, \mathrm{CH}_{\text {arom. }}\right), 7.34$ (dd, $\left.2 \mathrm{H}, J=7.4 \mathrm{~Hz}, J=7.4 \mathrm{~Hz}, \mathrm{CH}_{\text {arom. }}\right), 7.28$ (s, $\left.1 \mathrm{H}, \mathrm{CH}-1\right)$, 4.36-4.24 (m, 3H, $\mathrm{CH}_{2}-3$ and $\left.\mathrm{CH}-4\right), 3.88\left(\mathrm{~s}, 3 \mathrm{H}, \mathrm{CH}_{3}-2\right) .{ }^{13} \mathrm{C}-\mathrm{NMR}\left(125 \mathrm{MHz}\right.$, DMSO- $\left.d_{6}, \delta\right): 160.1(\mathrm{C}=\mathrm{O})$, $153.4(\mathrm{C}=\mathrm{O}), 143.7\left(2 \mathrm{C}, \mathrm{C}_{\mathrm{q}}\right), 140.8\left(2 \mathrm{C}, \mathrm{C}_{\mathrm{q}}\right), 137.4\left(\mathrm{C}_{\mathrm{q}}\right), 132.3\left(\mathrm{C}_{\mathrm{q}}\right), 127.7(2 \mathrm{C}, \mathrm{CH}), 127.2(2 \mathrm{C}, \mathrm{CH}), 125.5$ 
(2C, CH), $120.2(2 \mathrm{C}, \mathrm{CH}), 113.3(\mathrm{CH}), 66.2\left(\mathrm{CH}_{2}\right), 46.5(\mathrm{CH}), 35.5\left(\mathrm{CH}_{3}\right)$. HRMS$-\mathbf{E S I}^{+}(\mathrm{m} / \mathrm{z}):[\mathrm{M}+\mathrm{H}]^{+}$ calcd for $\mathrm{C}_{20} \mathrm{H}_{17} \mathrm{~N}_{3} \mathrm{O}_{4} \mathrm{H}, 364.1292$; found, 364.1284.

\section{Synthesis of ImPy-OH (31) and Fmoc- $\gamma-O H$ (9)}

This synthesis was reported before by Sekine et al. ${ }^{[\mathrm{xv}]}$ and Herdewijn et al. ${ }^{[\mathrm{xvi}]}$. We followed the same approach with minor modifications.

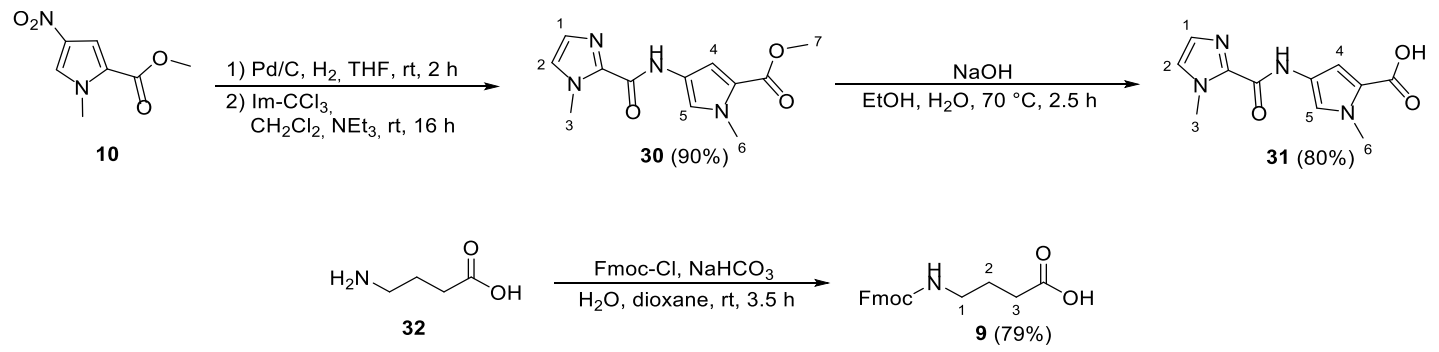

Scheme S3: Synthesis route of ImPy-OH (31) and Fmoc- $\gamma-\mathrm{OH}(\mathbf{9})$.

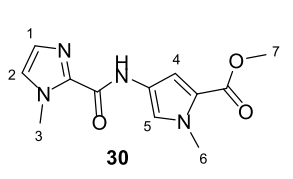

methyl 1-methyl-4-(1-methyl-1H-imidazole-2-carboxamido)-1H-pyrrole-2carboxylate (30): $\mathrm{Pd} / \mathrm{C}(10 \%$ dry, $400 \mathrm{mg}, 40 \% \mathrm{w} / \mathrm{w})$ was added to a solution of nitroPy-OMe $(1.00 \mathrm{~g}, 5.43 \mathrm{mmol})$ in THF $(10.0 \mathrm{~mL})$. The mixture was stirred under hydrogen atmosphere for $2 \mathrm{~h}$. The catalyst was removed by filtration through celite and the solvent removed under reduced pressure. The residue was dissolved in $\mathrm{CH}_{2} \mathrm{Cl}_{2}(15 \mathrm{~mL})$ and Im$\mathrm{CCl}_{3}(24,1.24 \mathrm{~g}, 5.43 \mathrm{mmol})$ and $\mathrm{NEt}_{3}(2.27 \mathrm{~mL}, 16.3 \mathrm{mmol})$ added. The reaction was stirred for $16 \mathrm{~h}$, the solvent removed and the crude purified by column chromatography (silica, $\mathrm{CH}_{2} \mathrm{Cl}_{2} / \mathrm{MeOH}$ 100:0 $\rightarrow$ 100:1 $\rightarrow 50: 1)$ to yield the desired product $(\mathbf{3 0}, 1.29 \mathrm{~g}, 4.91 \mathrm{mmol}, 90 \%)$ as pale brown solid. The characterization is in agreement with the literature. ${ }^{[x v i i]} \mathbf{R}_{f}=0.27$ (n-pentane/EtOAc 1:1). ${ }^{1} \mathbf{H}-\mathbf{N M R}\left(300 \mathrm{MHz}, \mathrm{CDCl}_{3}, \delta\right)$ : $9.12(\mathrm{~s}, 1 \mathrm{H}, \mathrm{N} H), 7.40$ (d, 1H, $J=2.0 \mathrm{~Hz}, \mathrm{CH}-2), 7.03$ (d, 1H, J=0.9 Hz, CH-5), 6.98 (d, 1H, J=0.9 Hz, $\mathrm{CH}-4), 6.80(\mathrm{~d}, 1 \mathrm{H}, \mathrm{J}=2.0 \mathrm{~Hz}, \mathrm{CH}-1), 4.09$ (s, 3H, $\left.\mathrm{CH}_{3}-6\right), 3.90\left(\mathrm{~s}, 3 \mathrm{H}, \mathrm{CH}_{3}-3\right), 3.80$ (s, 3H, $\left.\mathrm{CH}_{3}-7\right) .{ }^{13} \mathrm{C}-$ NMR $\left(75 \mathrm{MHz}, \mathrm{CDCl}_{3}, \delta\right): 161.6(\mathrm{C}=\mathrm{O}), 156.4(\mathrm{C}=\mathrm{O}), 139.0\left(\mathrm{C}_{\mathrm{q}}\right), 127.9(\mathrm{CH}), 125.9(\mathrm{CH}), 121.4\left(\mathrm{C}_{\mathrm{q}}\right)$, $120.7(\mathrm{CH}), 120.2\left(\mathrm{C}_{\mathrm{q}}\right), 108.4(\mathrm{CH}), 51.2\left(\mathrm{CH}_{3}\right), 36.9\left(\mathrm{CH}_{3}\right), 35.8\left(\mathrm{CH}_{3}\right)$. HRMS-ESI ${ }^{+}(\mathrm{m} / \mathrm{z}):[\mathrm{M}+\mathrm{H}]^{+}$calcd for $\mathrm{C}_{12} \mathrm{H}_{14} \mathrm{~N}_{4} \mathrm{O}_{3} \mathrm{H}, 263.1144$; found, 263.1139.

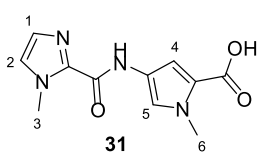

1-methyl-4-(1-methyl-1H-imidazole-2-carboxamido)-1H-pyrrole-2-carboxylic acid (31): ImPy-OMe $(723 \mathrm{mg}, 2.75 \mathrm{mmol})$ was dissolved in EtOH $(3.0 \mathrm{~mL})$ and $0.6 \mathrm{M}$ $\mathrm{NaOH}\left(\mathrm{aq}, 5.0 \mathrm{~mL}\right.$ ) and stirred at $70{ }^{\circ} \mathrm{C}$ for $2.5 \mathrm{~h}$. The $\mathrm{EtOH}$ was removed under reduced pressure and the aqueous phase extracted with $\mathrm{CH}_{2} \mathrm{Cl}_{2}$. The aqueous layers were acidified with $1 \mathrm{M} \mathrm{HCl} \mathrm{(aq)} \mathrm{to} \mathrm{pH} 3$, filtered, washed with deionized $\mathrm{H}_{2} \mathrm{O}$ and freeze dried to yield the desired product $(\mathbf{3 1}, 544 \mathrm{mg}, 2.19 \mathrm{mmol}, 80 \%)$ as grey solid. The characterization is in agreement with the literature. ${ }^{[\mathrm{xv}]} \mathbf{R}_{f}=0.42$ (EtOAc). ${ }^{1} \mathbf{H}-\mathbf{N M R}\left(300 \mathrm{MHz}, \mathrm{DMSO}-d_{6}, \delta\right): 12.20$ (br s, $\left.1 \mathrm{H}, \mathrm{COOH}\right), 10.47(\mathrm{~s}, 1 \mathrm{H}$, $\mathrm{NH}), 7.47$ (s, 1H, CH-2), 7.39 (s, 1H, CH-5), 7.04 (s, 1H, CH-4), 6.98 (s, 1H, CH-1), 3.98 (s, 3H, CH $\left.H_{3}-6\right)$, $3.82\left(\mathrm{~s}, 3 \mathrm{H}, \mathrm{CH}_{3}-3\right) .{ }^{13} \mathrm{C}-\mathrm{NMR}\left(75 \mathrm{MHz}, \mathrm{DMSO}-d_{6}, \delta\right): 161.9(\mathrm{C}=\mathrm{O}), 156.0(\mathrm{C}=\mathrm{O}), 138.6\left(\mathrm{C}_{\mathrm{q}}\right), 126.9(\mathrm{CH})$, $126.3(\mathrm{CH}), 121.9\left(\mathrm{C}_{\mathrm{q}}\right), 120.4(\mathrm{CH}), 119.7\left(\mathrm{C}_{\mathrm{q}}\right), 108.9(\mathrm{CH}), 36.1\left(\mathrm{CH}_{3}\right), 35.1\left(\mathrm{CH}_{3}\right)$. HRMS$-\mathbf{E S I}^{+}(\mathrm{m} / \mathrm{z})$ : $[\mathrm{M}+\mathrm{H}]^{+}$calcd for $\mathrm{C}_{11} \mathrm{H}_{12} \mathrm{~N}_{4} \mathrm{O}_{3} \mathrm{H}, 249.0982$; found, 249.0978 . 


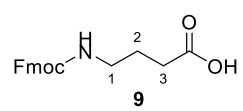

4-(((9H-fluoren-9-yl)methoxy)carbonyl)amino)butanoic acid (9): 4-Aminobutanoic acid $(502 \mathrm{mg}, 4.87 \mathrm{mmol})$ was dissolved in deionized water $(24 \mathrm{~mL})$ and 1,4-dioxane $(12 \mathrm{~mL})$. Fmoc-Cl (1.38 g, $5.33 \mathrm{mmol})$ and $\mathrm{NaHCO}_{3}(1.92 \mathrm{~g} 22.9 \mathrm{mmol})$ were added and the mixture stirred at $\mathrm{rt}$ for $3.5 \mathrm{~h}$. The mixture was poured into deionized water $(50 \mathrm{~mL})$ and extracted with $\mathrm{Et}_{2} \mathrm{O}(3 \times 50 \mathrm{~mL})$. The aqueous layer was acidified with $2 \mathrm{M} \mathrm{HCl}(\mathrm{aq})$ to $\mathrm{pH} 3$ and the remaining precipitate filtered and dried in vacuo to yield the desired product $(9,1.25 \mathrm{~g}, 3.85 \mathrm{mmol}, 79 \%)$ as white solid. The characterization is in agreement with the literature. ${ }^{[\mathrm{xviii}]} \mathbf{R}_{\boldsymbol{f}}=0.83\left(\mathrm{CH}_{2} \mathrm{Cl}_{2} / \mathrm{MeOH} 1: 1\right) .{ }^{1} \mathbf{H}-\mathbf{N M R}(300 \mathrm{MHz}$, DMSO- $d_{6}, \delta$ ): 11.62 (br s, $\left.1 \mathrm{H}, \mathrm{COOH}\right), 7.88$ (d, $\left.2 \mathrm{H}, J=7.3 \mathrm{~Hz}, \mathrm{CH}_{\text {arom. }}\right), 7.69$ (d, 2H, $J=7.3 \mathrm{~Hz}, \mathrm{C} H_{\text {arom. }}$ ), $7.45-7.37$ (m, 2H, CHarom.), $7.36-7.29$ (m, 3H, CH $\left.H_{\text {arom. }}, \mathrm{NH}\right), 4.29$ (d, 2H, J=6.7 Hz, CH $\mathrm{CH}_{2}$-Fmoc), $4.25-$ 4.16 (m, 1H, CH-Fmoc), $3.04-2.95$ (m, 2H, CH$\left.H_{2}-1\right), 2.20$ (t, 2H, J=7.4 Hz, CH2-3), $1.69-1.55$ (m, 2H, $\left.\mathrm{CH}_{2}-2\right) .{ }^{13} \mathrm{C}-\mathrm{NMR}\left(75 \mathrm{MHz}, \mathrm{DMSO}-d_{6}, \delta\right): 174.2(\mathrm{C}=\mathrm{O}), 156.1(\mathrm{C}=\mathrm{O}), 143.9\left(2 \mathrm{C}, \mathrm{C}_{\mathrm{q}}\right), 140.7\left(2 \mathrm{C}, \mathrm{C}_{\mathrm{q}}\right)$, $127.5(2 \mathrm{C}, \mathrm{CH}), 127.0(2 \mathrm{C}, \mathrm{CH}), 125.1(2 \mathrm{C}, \mathrm{CH}), 120.1(2 \mathrm{C}, \mathrm{CH}), 65.2\left(\mathrm{CH}_{2}\right), 46.7(\mathrm{CH}), 39.7\left(\mathrm{CH}_{2}\right), 31.1$ $\left(\mathrm{CH}_{2}\right), 29.9\left(\mathrm{CH}_{2}\right)$. HRMS-ESI ${ }^{+}(\mathrm{m} / \mathrm{z})$ : $[\mathrm{M}+\mathrm{Na}]^{+}$calcd for $\mathrm{C}_{19} \mathrm{H}_{19} \mathrm{~N}_{1} \mathrm{O}_{4} \mathrm{Na}, 348.1206$; found, 348.1201 . 


\section{Synthesis of Mtt-building blocks}

This synthesis is based in the preparation of the Mtt-protected methyl esters to yield the final Mtt-building blocks 2, 3, 4, and 5, which are used in the solid phase synthesis.
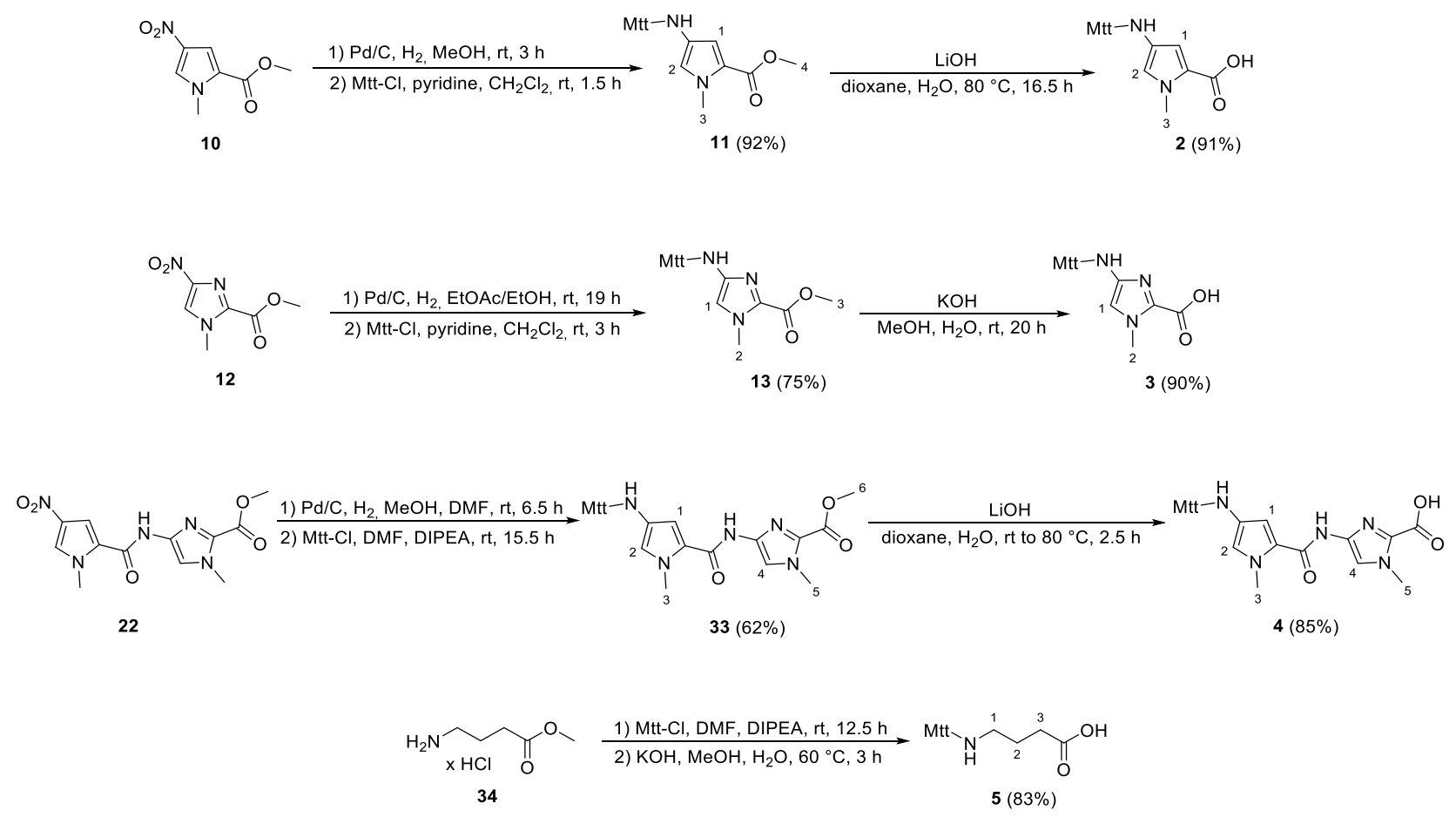

Scheme S4: Synthesis route of Mtt-Py-OH (2), Mtt-Im-OH (3) Mtt-PyIm-OH (4) and Mtt- $-\mathrm{OH}$ (5).

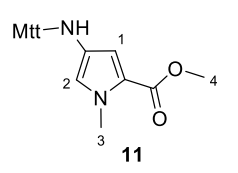

methyl 4-((diphenyl(p-tolyl)methyl)amino)-1-methyl-1H-pyrrole-2-carboxylate (11): Nitro-Py-OMe (10, $300 \mathrm{mg}, 1.63 \mathrm{mmol})$ was dissolved in $\mathrm{MeOH}(7.0 \mathrm{~mL}) . \mathrm{Pd} / \mathrm{C}(10 \%$ dry, $75 \mathrm{mg}, 25 \% \mathrm{w} / \mathrm{w}$ ) was added and the reaction mixture stirred under hydrogen atmosphere. After $3 \mathrm{~h}$, it was filtered over celite and the solvent removed. Under inert atmosphere, the residue was dissolved in $\mathrm{CH}_{2} \mathrm{Cl}_{2}(2.4 \mathrm{~mL})$ and pyridine $(2.0 \mathrm{~mL})$. At $0{ }^{\circ} \mathrm{C}, \mathrm{MttCl}(477 \mathrm{mg}, 1.63 \mathrm{mmol})$ dissolved in $\mathrm{CH}_{2} \mathrm{Cl}_{2}(1.2 \mathrm{~mL})$ and pyridine $(1.0 \mathrm{~mL})$ was added dropwise for $30 \mathrm{~min}$. After $1 \mathrm{~h}$ at $\mathrm{rt}$, the solvent was removed under reduced pressure, the crude co-evaporated with EtOAc $(3 \times 10 \mathrm{~mL})$ and purified via flash column chromatography (silica, $\mathrm{CH}_{2} \mathrm{Cl}_{2} / \mathrm{NEt}_{3}$ 100:2). Mtt-Py-OMe (11, $616 \mathrm{mg}, 1.50 \mathrm{mmol}, 92 \%$ ) was obtained as pale yellow solid. $\mathbf{R}_{f}=0.52\left(\mathrm{CH}_{2} \mathrm{Cl}_{2} / \mathrm{NEt}_{3}\right.$ 100:1). ${ }^{1} \mathbf{H}-\mathbf{N M R}\left(300 \mathrm{MHz}, \mathrm{CD}_{3} \mathrm{OD}, \delta\right): 7.41-$ $7.36\left(\mathrm{~m}, 4 \mathrm{H}, \mathrm{C} H_{\text {arom. }}\right), 7.28-7.14\left(\mathrm{~m}, 8 \mathrm{H}, \mathrm{C} H_{\text {arom. }}\right), 7.08-7.02\left(\mathrm{~m}, 2 \mathrm{H}, \mathrm{C} H_{\text {arom. }}\right), 6.12(\mathrm{~d}, 1 \mathrm{H}, J=2.1 \mathrm{~Hz}$, $\mathrm{CH}-2), 5.83$ (d, $1 \mathrm{H}, J=2.1 \mathrm{~Hz}, \mathrm{CH}-1), 3.65$ (s, 3H, $\left.\mathrm{CH}_{3}-4\right), 3.55$ (s, 3H, CH3 -3$), 2.28$ (s, 3H, CH3-Mtt). ${ }^{13}$ C-NMR $\left(75 \mathrm{MHz}, \mathrm{CD}_{3} \mathrm{OD}, \delta\right): 163.2(\mathrm{C}=\mathrm{O}), 147.6\left(2 \mathrm{C}, \mathrm{C}_{\mathrm{q}}\right), 144.4\left(\mathrm{C}_{\mathrm{q}}\right), 137.1\left(\mathrm{C}_{\mathrm{q}}\right), 133.3\left(\mathrm{C}_{\mathrm{q}}\right), 130.3$ $(4 \mathrm{C}, \mathrm{CH}), 130.3(2 \mathrm{C}, \mathrm{CH}), 129.2(2 \mathrm{C}, \mathrm{CH}), 128.5(4 \mathrm{C}, \mathrm{CH}), 127.4(2 \mathrm{C}, \mathrm{CH}), 120.9(\mathrm{CH}), 119.9\left(\mathrm{C}_{\mathrm{q}}\right), 111.1$ $(\mathrm{CH}), 73.1\left(\mathrm{C}_{\mathrm{q}}\right), 51.1\left(\mathrm{CH}_{3}\right), 36.4\left(\mathrm{CH}_{3}\right), 21.0\left(\mathrm{CH}_{3}\right)$. HRMS-ESI ${ }^{+}(\mathrm{m} / \mathrm{z}):[\mathrm{M}+\mathrm{H}]^{+}$calcd for $\mathrm{C}_{27} \mathrm{H}_{26} \mathrm{~N}_{2} \mathrm{O}_{2} \mathrm{H}$, 411.2067; found, 411.2062. FT-IR (neat) $\tilde{v}=3055(\mathrm{w}), 3022(\mathrm{w}), 2945(\mathrm{w}), 1698(\mathrm{~m}), 1571(\mathrm{w}), 1507(\mathrm{w})$, 1490 (w), 1443 (s), 1404 (w), 1368 (w), 1255 (m), 1209 (w), 1185 (w), 1151 (w), 1099 (s), 1054 (w), 1028 (w), 962 (w), 901 (w), 814 (m), 762 (m), 701 (s), 629 (w), 590 (w), 574 (w), $511($ w), 472 (w), 439 (w). 


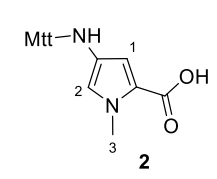

4-((diphenyl(p-tolyl)methyl)amino)-1-methyl-1H-pyrrole-2-carboxylic acid (2): MttPy-OMe (11, $275 \mathrm{mg}, 0.67 \mathrm{mmol})$ was dissolved in 1,4-dioxane $(18 \mathrm{~mL}), 1 \mathrm{M} \mathrm{LiOH}$ (aq, $5 \mathrm{~mL}$ ) added and the reaction mixture stirred for $16.5 \mathrm{~h}$ at $80{ }^{\circ} \mathrm{C}$. The solvent was removed and the crude purified over reverse phase silica column chromatography (Oasis HLB cc Vac Cartridge, $30 \mu \mathrm{m}$ particle size, Waters, USA; $\mathrm{H}_{2} \mathrm{O} / \mathrm{MeCN} / \mathrm{NEt}_{3}$ 100:0:0.1\% $\rightarrow$ 90:10:0.1\% $\rightarrow$ 75:25:0.1\% $\rightarrow$ 50:50:0.1\% $\rightarrow$ 0:100:0.1\%) to yield Mtt-Py-OH (2, $241 \mathrm{mg}, 0.61 \mathrm{mmol}, 91 \%)$ as pale yellow solid. $\mathbf{R}_{f}=$ $0.54\left(\mathrm{CH}_{2} \mathrm{Cl}_{2} / \mathrm{MeOH} / \mathrm{NEt}_{3}\right.$ 100:10:1). ${ }^{1} \mathbf{H}-\mathrm{NMR}$ (500 MHz, DMSO- $\left.d_{6}, \delta\right): 7.37-7.34$ (m, 4H, $\mathrm{CH}_{\text {arom. }}$ ), 7.25 - 7.21 (m, 6H, CH$\left.H_{\text {arom. }}\right), 7.16-7.12\left(\mathrm{~m}, 2 \mathrm{H}, \mathrm{C} H_{\text {arom. }}\right), 7.05-7.02\left(\mathrm{~m}, 2 \mathrm{H}, \mathrm{C} H_{\text {arom. }}\right), 5.69(\mathrm{~d}, 1 \mathrm{H}, J=2.1 \mathrm{~Hz}$, $\mathrm{CH}-2), 5.39$ (d, $1 \mathrm{H}, J=2.1 \mathrm{~Hz}, \mathrm{CH}-1), 5.13$ (s, $1 \mathrm{H}, \mathrm{NH}), 3.49$ (s, 3H, $\left.\mathrm{CH}_{3}-3\right), 2.24$ (s, 3H, $\left.\mathrm{CH}_{3}-\mathrm{Mtt}\right) .{ }^{13} \mathbf{C}-$ NMR $\left(125 \mathrm{MHz}, \mathrm{DMSO}-d_{6}, \delta\right): 166.1(\mathrm{C}=\mathrm{O}), 146.6\left(2 \mathrm{C}, \mathrm{C}_{\mathrm{q}}\right), 143.5\left(\mathrm{C}_{\mathrm{q}}\right), 134.8\left(\mathrm{C}_{\mathrm{q}}\right), 129.6\left(\mathrm{C}_{\mathrm{q}}\right), 129.0\left(\mathrm{C}_{\mathrm{q}}\right)$, $128.8(6 \mathrm{C}, \mathrm{CH}), 127.9(2 \mathrm{C}, \mathrm{CH}), 127.2(4 \mathrm{C}, \mathrm{CH}), 125.9(2 \mathrm{C}, \mathrm{CH}), 112.4(\mathrm{CH}), 106.0(\mathrm{CH}), 71.0\left(\mathrm{C}_{\mathrm{q}}\right), 35.2$ $\left(\mathrm{CH}_{3}\right), 20.5\left(\mathrm{CH}_{3}\right)$. HRMS-ESI ${ }^{+}(\mathrm{m} / \mathrm{z})$ : $[\mathrm{M}+\mathrm{Na}]^{+}$calcd for $\mathrm{C}_{26} \mathrm{H}_{24} \mathrm{~N}_{2} \mathrm{O}_{2} \mathrm{Na}, 419.1730$; found, 419.1725 . FTIR (neat) $\tilde{v}=3345(\mathrm{w}), 3081(\mathrm{w}), 3056(\mathrm{w}), 3022(\mathrm{w}), 2975(\mathrm{w}), 2921(\mathrm{w}), 2172(\mathrm{w}), 1562(\mathrm{~m}), 1489(\mathrm{w})$, 1438 (s), 1402 (w), 1364 (w), $1326(\mathrm{w}), 1284(\mathrm{w}), 1207(\mathrm{w}), 1185(\mathrm{w}), 1152(\mathrm{w}), 1119(\mathrm{w}), 1030(\mathrm{w}), 901$ $(\mathrm{w}), 814(\mathrm{w}), 771(\mathrm{~m}), 700(\mathrm{~s}), 626(\mathrm{w}), 576(\mathrm{w}), 511(\mathrm{w}), 492(\mathrm{w}), 472(\mathrm{w}), 450(\mathrm{w})$.

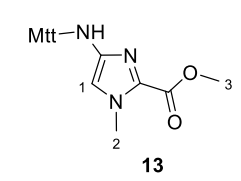

methyl 4-((diphenyl(p-tolyl)methyl)amino)-1-methyl-1H-imidazole-2-carboxylate (13): Nitro-Im-OMe (12, $506 \mathrm{mg}, 2.96 \mathrm{mmol})$ was dissolved in EtOH/EtOAc 1:1 (10 mL). $\mathrm{Pd} / \mathrm{C}(10 \%$ dry, $38 \mathrm{mg}, 8 \% \mathrm{w} / \mathrm{w})$ was added and the reaction mixture stirred under hydrogen atmosphere. After $19 \mathrm{~h}$, it was filtered over celite and the solvent removed. Under inert atmosphere, the residue was dissolved in $\mathrm{CH}_{2} \mathrm{Cl}_{2}(3.6 \mathrm{~mL})$ and pyridine $(2.4 \mathrm{~mL})$ and $\mathrm{MttCl}(867 \mathrm{mg}$, $2.96 \mathrm{mmol}$ ) was added. After $3 \mathrm{~h}$ at $\mathrm{rt}$, the solvent was removed under reduced pressure, the crude coevaporated with EtOAc (3 x $10 \mathrm{~mL})$ and purified via flash column chromatography (silica, $\mathrm{CH}_{2} \mathrm{Cl}_{2} / \mathrm{MeOH} / \mathrm{NEt}_{3}$ 100:0:1 $\rightarrow$ 100:1:1 $\rightarrow$ 10:1:1). Mtt-Im-OMe (13, $908 \mathrm{mg}, 2.21 \mathrm{mmol}$, 75\%) was preserved as pale yellow solid. $\mathbf{R}_{\boldsymbol{f}}=0.24\left(\mathrm{CH}_{2} \mathrm{Cl}_{2} / \mathrm{NEt}_{3}\right.$ 100:1). ${ }^{1} \mathbf{H}-\mathbf{N M R}\left(300 \mathrm{MHz}, \mathrm{CD}_{3} \mathrm{OD}, \delta\right): 7.40-7.33$ (m, 4H, CH $\left.H_{\text {arom. }}\right), 7.29-7.15$ (m, 8H, $\left.\mathrm{CH}_{\text {arom. }}\right), 7.11-7.04$ (m, 2H, $\left.\mathrm{C} H_{\text {arom. }}\right), 5.46$ (s, $\left.1 \mathrm{H}, \mathrm{CH}-1\right), 3.81$ (s, 3H, $\mathrm{CH}_{3}-3$ ), 3.59 (s, 3H, CH-2 ), 2.28 (s, 3H, CH -Mtt). ${ }^{13} \mathbf{C}-\mathbf{N M R}\left(75 \mathrm{MHz}, \mathrm{CD}_{3} \mathrm{OD}, \delta\right): 160.0$ (C=O), 147.1 $\left(\mathrm{C}_{\mathrm{q}}\right), 146.7\left(2 \mathrm{C}, \mathrm{C}_{\mathrm{q}}\right), 143.5\left(\mathrm{C}_{\mathrm{q}}\right), 137.7\left(\mathrm{C}_{\mathrm{q}}\right), 131.6\left(\mathrm{C}_{\mathrm{q}}\right), 130.0(6 \mathrm{C}, \mathrm{CH}), 129.5(2 \mathrm{C}, \mathrm{CH}), 128.9(4 \mathrm{C}, \mathrm{CH})$, $127.9(2 \mathrm{C}, \mathrm{CH}), 111.7(\mathrm{CH}), 72.1\left(\mathrm{C}_{\mathrm{q}}\right), 52.3\left(\mathrm{CH}_{3}\right), 35.8\left(\mathrm{CH}_{3}\right), 20.9\left(\mathrm{CH}_{3}\right)$. HRMS-ESI ${ }^{+}(\mathrm{m} / \mathrm{z}):[\mathrm{M}+\mathrm{H}]^{+}$ calcd for $\mathrm{C}_{26} \mathrm{H}_{25} \mathrm{~N}_{3} \mathrm{O}_{2} \mathrm{H}, 412.2031$; found, 412.2023. FT-IR (neat) $\tilde{v}=3252$ (w), 3055 (w), 3022 (w), 2947 (w), 1704 (s), 1560 (m), 1485 (w), 1443 (s), 1412 (w), 1271 (m), 1199 (m), 1121 (s), 1067 (w), 1052 (w), $1014(\mathrm{w}), 966(\mathrm{w}), 928(\mathrm{w}), 900(\mathrm{w}), 843(\mathrm{w}), 816(\mathrm{~m}), 788(\mathrm{w}), 749(\mathrm{w}), 701(\mathrm{~s}), 629(\mathrm{~m}), 575(\mathrm{w}), 538$ (w), $513(\mathrm{w}), 474(\mathrm{w}), 438(\mathrm{w})$.

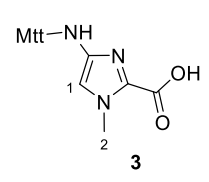

4-((diphenyl(p-tolyl)methyl)amino)-1-methyl-1H-imidazole-2-carboxylic acid (3): MttIm-OMe (13, $423 \mathrm{mg}, 1.03 \mathrm{mmol})$ was dissolved in $\mathrm{MeOH}(3.4 \mathrm{~mL}), 1 \mathrm{M} \mathrm{KOH}$ (aq, $1.7 \mathrm{~mL}$ ) added and the reaction mixture stirred for $20 \mathrm{~h}$ at $\mathrm{rt}$. At $0{ }^{\circ} \mathrm{C}$, it was acidified until $\mathrm{pH} 4$ with $10 \% \mathrm{AcOH}(\mathrm{aq})$. The product was extracted with $\mathrm{CHCl}_{3}(4 \times 50 \mathrm{~mL})$, washed with $10 \% \mathrm{AcOH}(3 \times 50 \mathrm{~mL})$ and the combined organic layers dried over $\mathrm{MgSO}_{4}$. The solvent was removed to yield Mtt-Im-OH (3, $368 \mathrm{mg}, 0.93 \mathrm{mmol}, 90 \%)$ as pale yellow solid. $\mathbf{R}_{\boldsymbol{f}}=0.51\left(\mathrm{CH}_{2} \mathrm{Cl}_{2} / \mathrm{MeOH} / \mathrm{NEt}_{3}\right.$ 100:10:1). ${ }^{1} \mathbf{H}-\mathbf{N M R}\left(300 \mathrm{MHz}, \mathrm{CDCl}_{3}, \delta\right): 8.43$ (br s, $\left.1 \mathrm{H}, \mathrm{NH}\right), 7.41-7.34$ (m, 4H, $\left.\mathrm{CH}_{\text {arom. }}\right), 7.27$ - 7.16 (m, 8H, CH $\left.H_{\text {arom. }}\right), 7.07-7.00\left(\mathrm{~m}, 2 \mathrm{H}, \mathrm{C} H_{\text {arom. }}\right), 4.87(\mathrm{~s}, 1 \mathrm{H}, \mathrm{CH}-1), 3.65\left(\mathrm{~s}, 3 \mathrm{H}, \mathrm{CH}_{3}-2\right), 2.28\left(\mathrm{~s}, 3 \mathrm{H}, \mathrm{CH}_{3}-\right.$ Mtt). ${ }^{13} \mathbf{C}-N M R\left(75 \mathrm{MHz}, \mathrm{CDCl}_{3}, \delta\right): 158.9(\mathrm{C}=\mathrm{O}), 144.7\left(2 \mathrm{C}, \mathrm{C}_{\mathrm{q}}\right), 141.4\left(\mathrm{C}_{\mathrm{q}}\right), 139.6\left(\mathrm{C}_{\mathrm{q}}\right), 136.5\left(\mathrm{C}_{\mathrm{q}}\right), 133.7$ $\left(\mathrm{C}_{\mathrm{q}}\right), 129.3(6 \mathrm{C}, \mathrm{CH}), 128.6(2 \mathrm{C}, \mathrm{CH}), 127.8(4 \mathrm{C}, \mathrm{CH}), 126.9(2 \mathrm{C}, \mathrm{CH}), 104.0(\mathrm{CH}), 71.6\left(\mathrm{C}_{\mathrm{q}}\right), 34.9\left(\mathrm{CH}_{3}\right)$, $21.1\left(\mathrm{CH}_{3}\right)$. HRMS-ESI ${ }^{+}(\mathrm{m} / \mathrm{z})$ : $[\mathrm{M}+\mathrm{H}]^{+}$calcd for $\mathrm{C}_{25} \mathrm{H}_{23} \mathrm{~N}_{3} \mathrm{O}_{2} \mathrm{H}, 398.1863$; found, 398.1865. FT-IR (neat) $\tilde{v}=3167(\mathrm{w}), 2921(\mathrm{w}), 1628(\mathrm{~m}), 1534(\mathrm{w}), 1511(\mathrm{w}), 1492(\mathrm{w}), 1444(\mathrm{w}), 1322(\mathrm{~m}), 1186(\mathrm{w}), 1159(\mathrm{w})$, 
$1123(w), 1094(w), 1070(w), 1031(w), 1001(w), 941(w), 900(w), 815(m), 798(w), 758(w), 701(s)$, $629(\mathrm{~m}), 576(\mathrm{w}), 541(\mathrm{w}), 502(\mathrm{w}), 438(\mathrm{w})$.

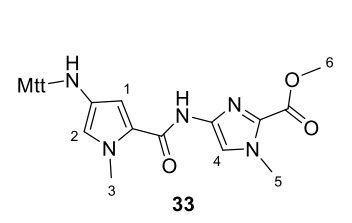

methyl 4-(4-((diphenyl(p-tolyl)methyl)amino)-1-methyl-1H-pyrrole-2carboxamido)-1-methyl-1H-imidazole-2-carboxylate (33): To a solution of nitro-PyIm-OMe (300 mg, $0.98 \mathrm{mmol})$ in $\mathrm{MeOH}(7.0 \mathrm{~mL})$ and DMF $(0.5 \mathrm{~mL})$, $\mathrm{Pd} / \mathrm{C}(10 \%$ dry, $100 \mathrm{mg}, 33 \% \mathrm{w} / \mathrm{w})$ was added and the mixture stirred under hydrogen atmosphere for $6.5 \mathrm{~h}$. The catalyst was removed by filtration through celite and the solvent removed under reduced pressure. The residue was dissolved in DMF (1.8 mL), and DIPEA $(268 \mu \mathrm{L}, 1.54 \mathrm{mmol})$ was added. At $0{ }^{\circ} \mathrm{C}$, Mtt-Cl $(220 \mathrm{mg}, 0.75 \mathrm{mmol})$ dissolved in DMF (1.2 mL) and DIPEA $(137 \mu \mathrm{L}, 0.79 \mathrm{mmol})$ was added dropwise over $10 \mathrm{~min}$. The reaction was stirred at $\mathrm{rt}$ for $15.5 \mathrm{~h}$ and the solvent removed under reduced pressure. The crude was purified by column chromatography (silica, $\left.\mathrm{CH}_{2} \mathrm{Cl}_{2} / \mathrm{NEt}_{3} 100: 1\right)$ to yield the desired product $(\mathbf{3 3}, 323 \mathrm{mg}, 0.61 \mathrm{mmol}, 62 \%)$ as pale yellow solid. $\mathbf{R}_{f}=$ 0.33 (n-pentane/EtOAc 1:1). ${ }^{1} \mathbf{H}-\mathbf{N M R}\left(300 \mathrm{MHz}, \mathrm{CDCl}_{3}, \delta\right): 7.44$ (s, 1H, CH-4), 7.43 -7.39 (m, 4H, $\left.\mathrm{C} H_{\text {arom. }}\right), 7.31-7.27$ (m, 2H, $\left.\mathrm{CH}_{\text {arom. }}\right), 7.25-7.20\left(\mathrm{~m}, 4 \mathrm{H}, \mathrm{C} H_{\text {arom. }}\right), 7.17-7.13\left(\mathrm{~m}, 2 \mathrm{H}, \mathrm{C} H_{\text {arom. }}\right), 7.07-$ 7.03 (m, 2H, CHarom.), 6.21 (d, $1 \mathrm{H}, J=2.0 \mathrm{~Hz}, \mathrm{CH}-2), 5.72$ (d, $1 \mathrm{H}, J=2.0 \mathrm{~Hz}, \mathrm{CH}-1), 3.95$ (s, 3H, $\left.\mathrm{CH}_{3}-5\right)$, 3.88 (s, 3H, CH $\left.H_{3}-6\right), 3.56$ (s, 3H, CH $\left.H_{3}-3\right), 2.28$ (s, 3H, $\left.\mathrm{CH}_{3}-\mathrm{Mtt}\right) .{ }^{13} \mathbf{C}-\mathbf{N M R}\left(75 \mathrm{MHz}, \mathrm{CDCl}_{3}, \delta\right.$ ): 161.0 $(\mathrm{C}=\mathrm{O}), 160.2(\mathrm{C}=\mathrm{O}), 147.6\left(2 \mathrm{C}, \mathrm{C}_{\mathrm{q}}\right), 144.4\left(\mathrm{C}_{\mathrm{q}}\right), 138.6\left(\mathrm{C}_{\mathrm{q}}\right), 137.1\left(\mathrm{C}_{\mathrm{q}}\right), 133.2\left(\mathrm{C}_{\mathrm{q}}\right), 132.8\left(\mathrm{C}_{\mathrm{q}}\right), 130.3(6 \mathrm{C}$, $\mathrm{CH}), 129.2(2 \mathrm{C}, \mathrm{CH}), 128.5(4 \mathrm{C}, \mathrm{CH}), 127.4(2 \mathrm{C}, \mathrm{CH}), 122.4\left(\mathrm{C}_{\mathrm{q}}\right), 119.9(\mathrm{CH}), 116.7(\mathrm{CH}), 107.4(\mathrm{CH})$, $73.2\left(\mathrm{C}_{\mathrm{q}}\right), 52.6\left(\mathrm{CH}_{3}\right), 36.4\left(2 \mathrm{C}, \mathrm{CH}_{3}\right), 21.0\left(\mathrm{CH}_{3}\right)$. HRMS-ESI ${ }^{+}(\mathrm{m} / \mathrm{z}):[\mathrm{M}+\mathrm{Na}]^{+}$calcd for $\mathrm{C}_{32} \mathrm{H}_{31} \mathrm{~N}_{5} \mathrm{O}_{3} \mathrm{Na}$, 556.2319; found, 556.2317. FT-IR (neat) $\tilde{v}=3322(\mathrm{w}), 3054(\mathrm{w}), 3022(\mathrm{w}), 2927(\mathrm{w}), 2851(\mathrm{w}), 1711(\mathrm{~m})$, $1650(\mathrm{~m}), 1559(\mathrm{w}), 1528(\mathrm{~m}), 1456(\mathrm{w}), 1431(\mathrm{~s}), 1401(\mathrm{w}), 1368(\mathrm{w}), 1301(\mathrm{w}), 1274(\mathrm{~m}), 1247(\mathrm{w}), 1215$ (w), $1187(\mathrm{w}), 1124(\mathrm{~s}), 1104(\mathrm{w}), 1055(\mathrm{w}), 1017(\mathrm{w}), 895(\mathrm{w}), 839(\mathrm{w}), 817(\mathrm{w}), 753(\mathrm{~m}), 701(\mathrm{~s}), 658$ (w), $629(\mathrm{w}), 589(\mathrm{w}), 511(\mathrm{w}), 442(\mathrm{w})$.

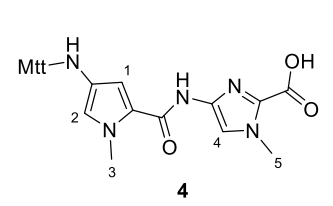

4-(4-((diphenyl(p-tolyl)methyl)amino)-1-methyl-1H-pyrrole-2-carboxamido)1-methyl-1H-imidazole-2-carboxylic acid (4): Mtt-PyIm-OMe (287 mg, $0.55 \mathrm{mmol})$ was dissolved in 1,4-dioxane $(14 \mathrm{~mL})$ and $1 \mathrm{M} \mathrm{LiOH}(\mathrm{aq}, 7 \mathrm{~mL})$ and stirred for $1 \mathrm{~h}$ at $80^{\circ} \mathrm{C}$ and $1.5 \mathrm{~h}$ at $\mathrm{rt}$. The dioxane was removed under reduced pressure and the crude purified over reverse phase silica column chromatography (Oasis HLB cc Vac Cartridge, $30 \mu \mathrm{m}$ particle size, Waters, USA; $\mathrm{H}_{2} \mathrm{O} / \mathrm{MeCN} / \mathrm{NEt}_{3}$ 100:0:0.1\% $\rightarrow$ 50:50:0.1\%). The product fractions were freeze dried to yield the desired product $(4,242 \mathrm{mg}, 0.47 \mathrm{mmol}$, $85 \%)$ as pale yellow solid. $\mathbf{R}_{f}=0.25\left(\mathrm{CH}_{2} \mathrm{Cl}_{2} / \mathrm{MeOH} / \mathrm{NEt}_{3}\right.$ 100:10:1). ${ }^{1} \mathbf{H}-\mathbf{N M R}\left(300 \mathrm{MHz}, \mathrm{DMSO}-d_{6}, \delta\right)$ : 10.09 (s, 1H, NH), $7.43-7.36$ (m, 4H, CHarom.), $7.31-7.24$ (m, 6H, $\left.\mathrm{CH}_{\text {arom. }}\right), 7.20-7.14$ (m, 2H, $\mathrm{C} H_{\text {arom. }}$ ), $7.11-7.06$ (m, 3H, $\mathrm{CH}_{\text {arom. }} \mathrm{CH}-4$ ), 7.00 (s, 1H, $\left.\mathrm{CH}-2\right), 5.40$ (s, 1H, CH-1), $5.24(\mathrm{~s}, 1 \mathrm{H}, \mathrm{NH}), 3.90(\mathrm{~s}, 3 \mathrm{H}$, $\left.\mathrm{CH}_{3}-5\right), 3.50$ (s, 3H, $\left.\mathrm{CH}_{3}-3\right), 2.25$ (s, 3H, CH $\left.3-\mathrm{Mtt}\right) .{ }^{13} \mathbf{C}-\mathbf{N M R}\left(75 \mathrm{MHz}, \mathrm{DMSO}-d_{6}, \delta\right): 161.7(\mathrm{C}=\mathrm{O}), 158.2$ $(\mathrm{C}=\mathrm{O}), 146.1\left(2 \mathrm{C}, \mathrm{C}_{\mathrm{q}}\right), 143.0\left(\mathrm{C}_{\mathrm{q}}\right), 140.4\left(\mathrm{C}_{\mathrm{q}}\right), 135.2\left(2 \mathrm{C}, \mathrm{C}_{\mathrm{q}}\right), 131.0\left(\mathrm{C}_{\mathrm{q}}\right), 128.8(4 \mathrm{C}, \mathrm{CH}), 128.7(2 \mathrm{C}, \mathrm{CH})$, $128.2(2 \mathrm{C}, \mathrm{CH}), 127.5(4 \mathrm{C}, \mathrm{CH}), 126.2(2 \mathrm{C}, \mathrm{CH}), 121.4\left(\mathrm{C}_{\mathrm{q}}\right), 116.0(\mathrm{CH}), 109.8(\mathrm{CH}), 106.5(\mathrm{CH}), 71.0$ $\left(\mathrm{C}_{\mathrm{q}}\right), 35.7\left(\mathrm{CH}_{3}\right), 34.2\left(\mathrm{CH}_{3}\right), 20.5\left(\mathrm{CH}_{3}\right)$. HRMS-ESI ${ }^{+}(\mathrm{m} / \mathrm{z})$ : $[\mathrm{M}+\mathrm{Li}]^{+}$calcd. for $\mathrm{C}_{31} \mathrm{H}_{29} \mathrm{~N}_{5} \mathrm{O}_{3} \mathrm{Li}, 526.2426$; found, 526.2423. FT-IR (neat) $\tilde{v}=3324$ (w), 3054 (w), $3022(w), 2928$ (w), $1622(w), 1574(w), 1538(w)$, $1461(\mathrm{w}), 1427$ (s), 1404 (w), $1345(\mathrm{~m}), 1309(\mathrm{w}), 1253(\mathrm{w}), 1217(\mathrm{w}), 1185(\mathrm{w}), 1156(\mathrm{w}), 1105(\mathrm{w}), 1054$ (w), $1029(\mathrm{w}), 898(\mathrm{w}), 846(\mathrm{w}), 813(\mathrm{w}), 753(\mathrm{~m}), 702(\mathrm{~s}), 663(\mathrm{w}), 632(\mathrm{w}), 587(\mathrm{w}), 508(\mathrm{w})$. 


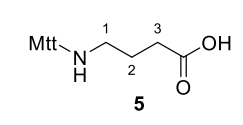

4-((diphenyl(p-tolyl)methyl)amino)butanoic acid (5): Methyl 4-aminobutyrate hydrochloride (300 mg, $1.95 \mathrm{mmol})$ was suspended in DMF (4.5 mL) and DIPEA (678 $\mu \mathrm{L}$, $3.89 \mathrm{mmol})$. At $0{ }^{\circ} \mathrm{C}, \mathrm{Mtt}-\mathrm{Cl}(520 \mathrm{mg}, 1.78 \mathrm{mmol})$ dissolved in DMF $(1.5 \mathrm{~mL})$ and DIPEA $(618 \mu \mathrm{L}, 3.55 \mathrm{mmol})$ was added dropwise over $5 \mathrm{~min}$. After $12.5 \mathrm{~h}$ at $\mathrm{rt}$, the solvent was removed under reduced pressure and the crude purified by column chromatography (silica, $\mathrm{CH}_{2} \mathrm{Cl}_{2} / \mathrm{NEt}_{3}$ 100:1). The product was dissolved $\mathrm{MeOH}(6.0 \mathrm{~mL})$ and $1 \mathrm{M} \mathrm{KOH}(\mathrm{aq}, 3 \mathrm{~mL})$ and stirred at $60{ }^{\circ} \mathrm{C}$ for $3 \mathrm{~h}$. The reaction mixture was extracted with $n$-pentane $(3 \times 50 \mathrm{~mL}), \mathrm{MeOH}$ was removed from the aqueous phase under reduced pressure and this phase acidified with $20 \% \mathrm{AcOH}$ (aq) until $\mathrm{pH} 4$. It was extracted with EtOAc (6 x $50 \mathrm{~mL}$ ), washed with brine and dried over $\mathrm{MgSO}_{4}$. The solvent was removed to yield the desired product $(\mathbf{5}, 581 \mathrm{mg}, 1.62 \mathrm{mmol}, 83 \%)$ as colourless solid. $\mathbf{R}_{f}=0.18$ (n-pentane/EtOAc 1:1). ${ }^{1} \mathbf{H}-\mathbf{N M R}(300 \mathrm{MHz}$, $\left.\mathrm{CD}_{3} \mathrm{OD}, \delta\right): 7.42-7.32$ (m, 10H, CH $\left.H_{\text {arom }}\right), 7.29-7.20$ (m, 4H, $\left.\mathrm{CH}_{\text {arom. }}\right), 2.73$ (t, $\left.2 \mathrm{H}, J=6.3 \mathrm{~Hz}, \mathrm{CH}_{2}-1\right), 2.45$ - 2.38 (m, 2H, CH2 -3$), 2.35$ (s, 3H, CH $\left.H_{3}-\mathrm{Mtt}\right), 1.90-1.80$ (m, 2H, $\left.\mathrm{CH}_{2}-2\right) .{ }^{13} \mathbf{C}-\mathbf{N M R}\left(75 \mathrm{MHz}, \mathrm{CD}_{3} \mathrm{OD}\right.$,

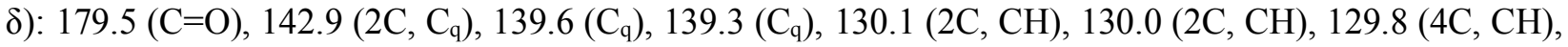
$129.5(4 \mathrm{C}, \mathrm{CH}), 129.1(2 \mathrm{C}, \mathrm{CH}), 74.8\left(\mathrm{C}_{\mathrm{q}}\right), 46.2\left(\mathrm{CH}_{2}\right), 35.4\left(\mathrm{CH}_{2}\right), 24.3\left(\mathrm{CH}_{2}\right), 21.0\left(\mathrm{CH}_{3}\right)$. HRMS-ESI ${ }^{+}$ $(\mathrm{m} / z)$ : $[\mathrm{M}+\mathrm{Na}]^{+}$calcd for $\mathrm{C}_{24} \mathrm{H}_{25} \mathrm{NO}_{2} \mathrm{Na}, 382.1778$; found, 382.1787. FT-IR (neat) $\tilde{v}=3022(\mathrm{w}), 2920(\mathrm{w})$, $2852(\mathrm{w}), 1707$ (w), $1562(\mathrm{w}), 1490(\mathrm{w}), 1446(\mathrm{~m}), 1403(\mathrm{w}), 1311(\mathrm{w}), 1190(\mathrm{w}), 1158(\mathrm{w}), 1103(\mathrm{w}), 1031$ (w), $1002(\mathrm{w}), 905(\mathrm{w}), 813(\mathrm{w}), 751(\mathrm{~m}), 702(\mathrm{~s}), 637(\mathrm{w}), 613(\mathrm{~m}), 571(\mathrm{w}), 533(\mathrm{w}), 496(\mathrm{w})$. 


\section{NMR and IR spectra}

\section{NMR spectra of previously reported compounds}
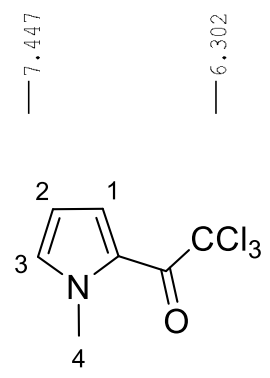

\section{9}

$300 \mathrm{MHz}, \mathrm{DMSO}-\mathrm{d} 6$

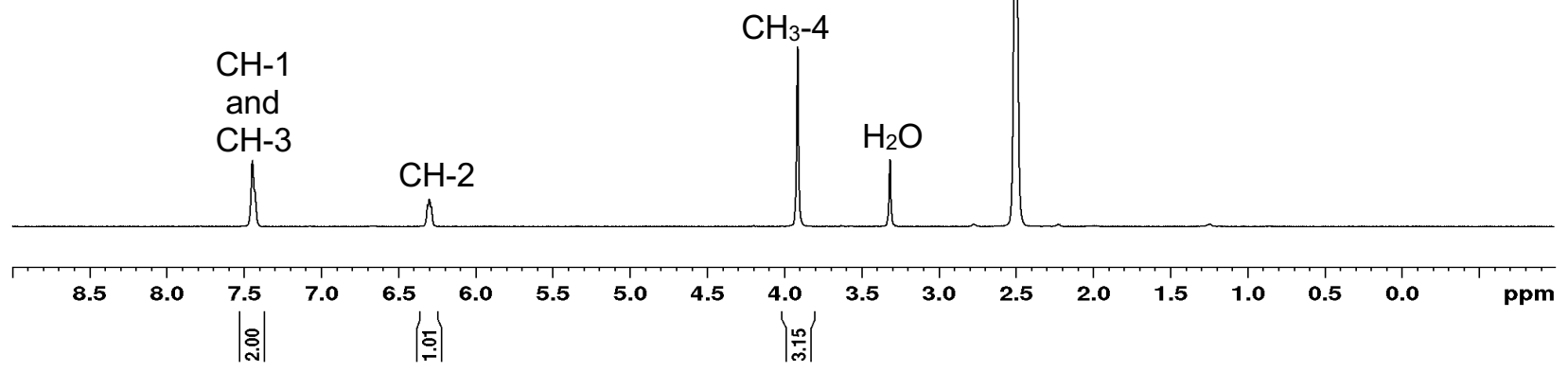

Figure S1: ${ }^{1} \mathrm{H}-\mathrm{NMR}\left(300 \mathrm{MHz}\right.$ ) spectrum of $\mathrm{Py} \mathrm{CCl}_{3}(\mathbf{1 9})$.<smiles>Cn1cc([N+](=O)[O-])cc1C(=O)C(Cl)(Cl)Cl</smiles>

20

$300 \mathrm{MHz}, \mathrm{DMSO}-\mathrm{d} 6$

\section{$\mathrm{CH}-2 \quad \mathrm{CH}-1$}
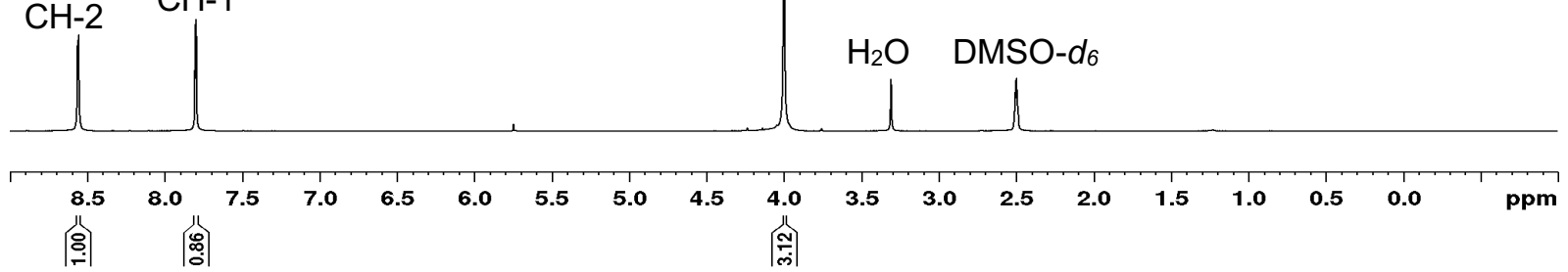

Figure S2: ${ }^{1} \mathrm{H}-\mathrm{NMR}(300 \mathrm{MHz})$ spectrum of $\mathrm{O}_{2} \mathrm{~N}-\mathrm{Py}-\mathrm{CCl}_{3}(\mathbf{2 0})$. 


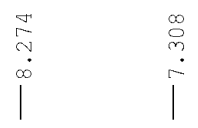<smiles>COC(=O)c1cc([N+](=O)[O-])cn1C</smiles>

10

$300 \mathrm{MHz}$, DMSO-d6

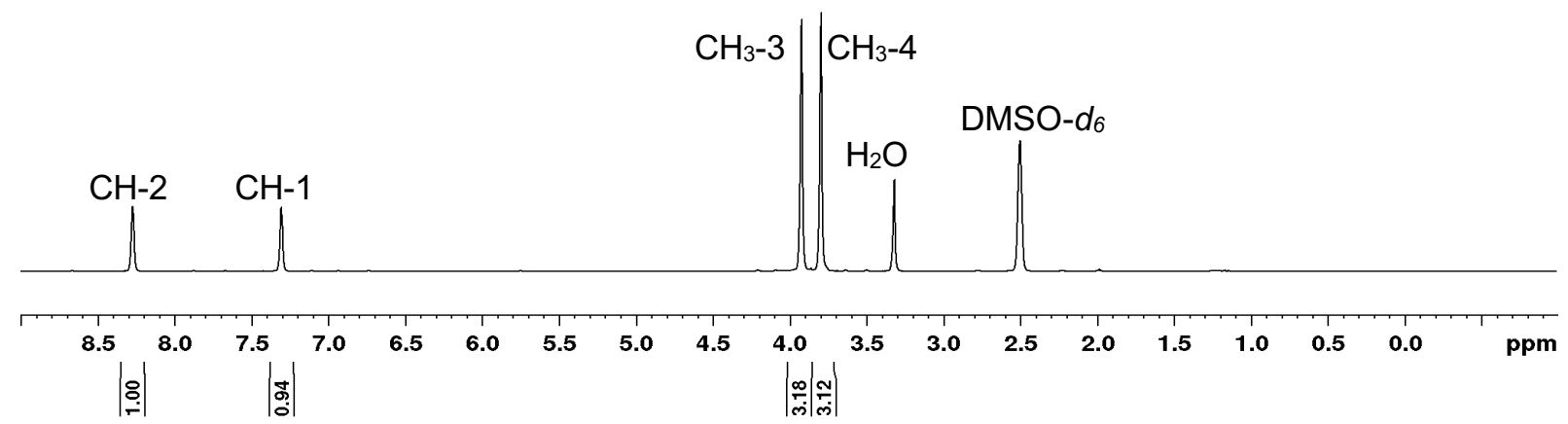

Figure S3: ${ }^{1} \mathrm{H}-\mathrm{NMR}\left(300 \mathrm{MHz}\right.$ ) spectrum of $\mathrm{O}_{2} \mathrm{~N}-\mathrm{Py}-\mathrm{OMe}(\mathbf{1 0})$.

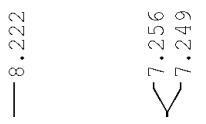

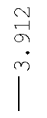<smiles>Cn1cc([N+](=O)[O-])cc1C(=O)O</smiles>

21

$300 \mathrm{MHz}, \mathrm{DMSO}-\mathrm{d} 6$

$\mathrm{CH}-2 \quad \mathrm{CH}-1$

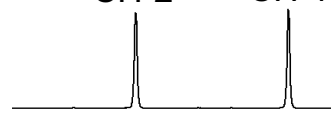

$\begin{array}{llll}8.5 & 8.0 & 7.5 & 7.0\end{array}$ $|\stackrel{0}{0}|$

Figure S4: ${ }^{1} \mathrm{H}-\mathrm{NMR}(300 \mathrm{MHz})$ spectrum of $\mathrm{O}_{2} \mathrm{~N}-\mathrm{Py}-\mathrm{OH}$ (21). 


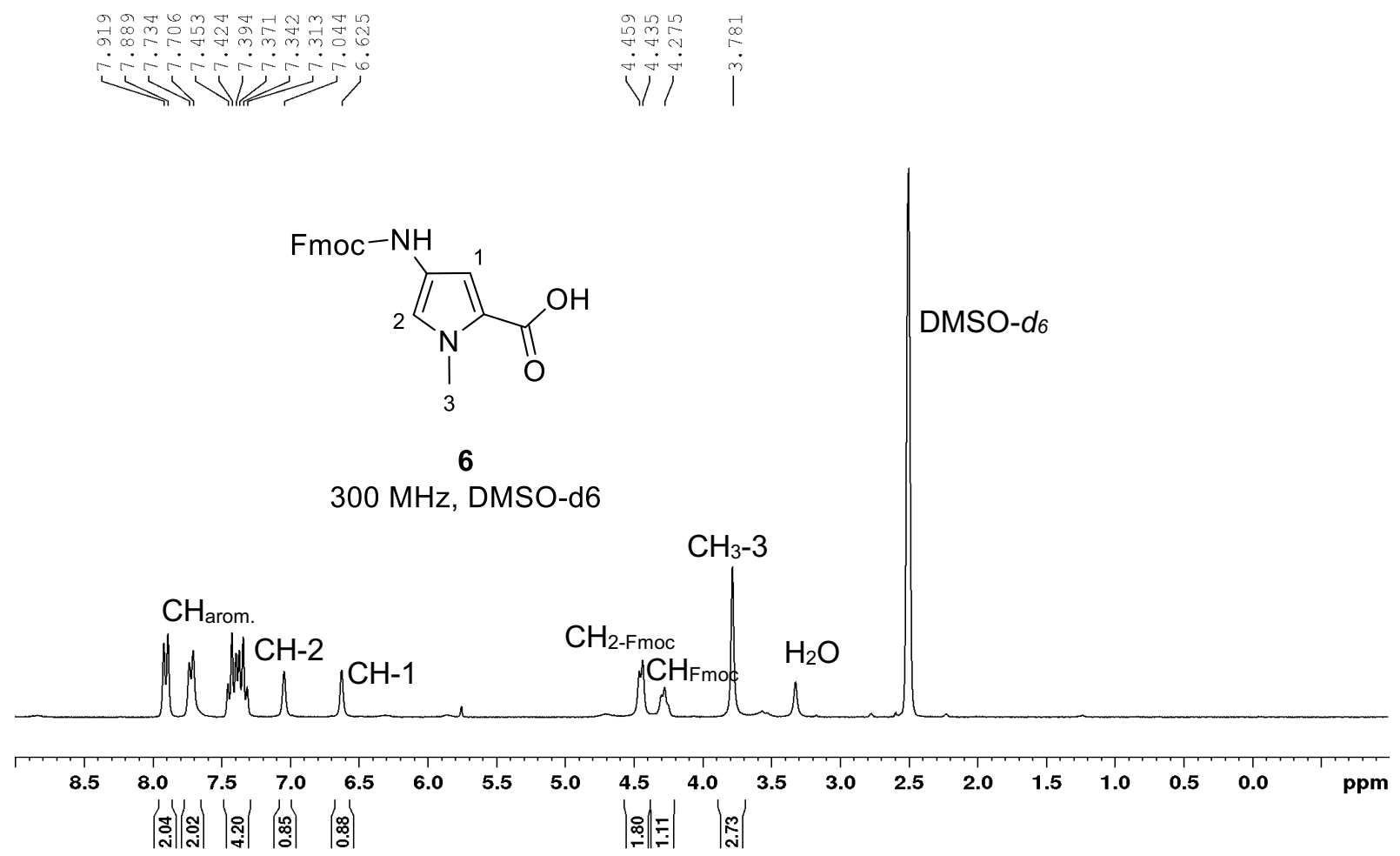

Figure S5: ${ }^{1} \mathrm{H}-\mathrm{NMR}(300 \mathrm{MHz})$ spectrum of Fmoc-Py-OH (6).
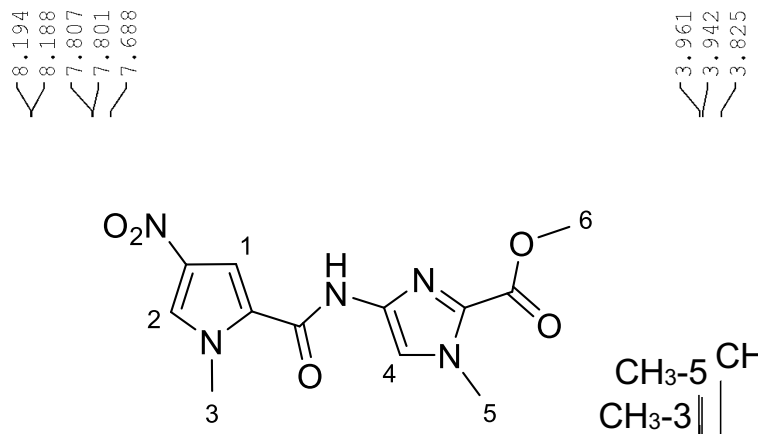

22

$300 \mathrm{MHz}$, DMSO-d6
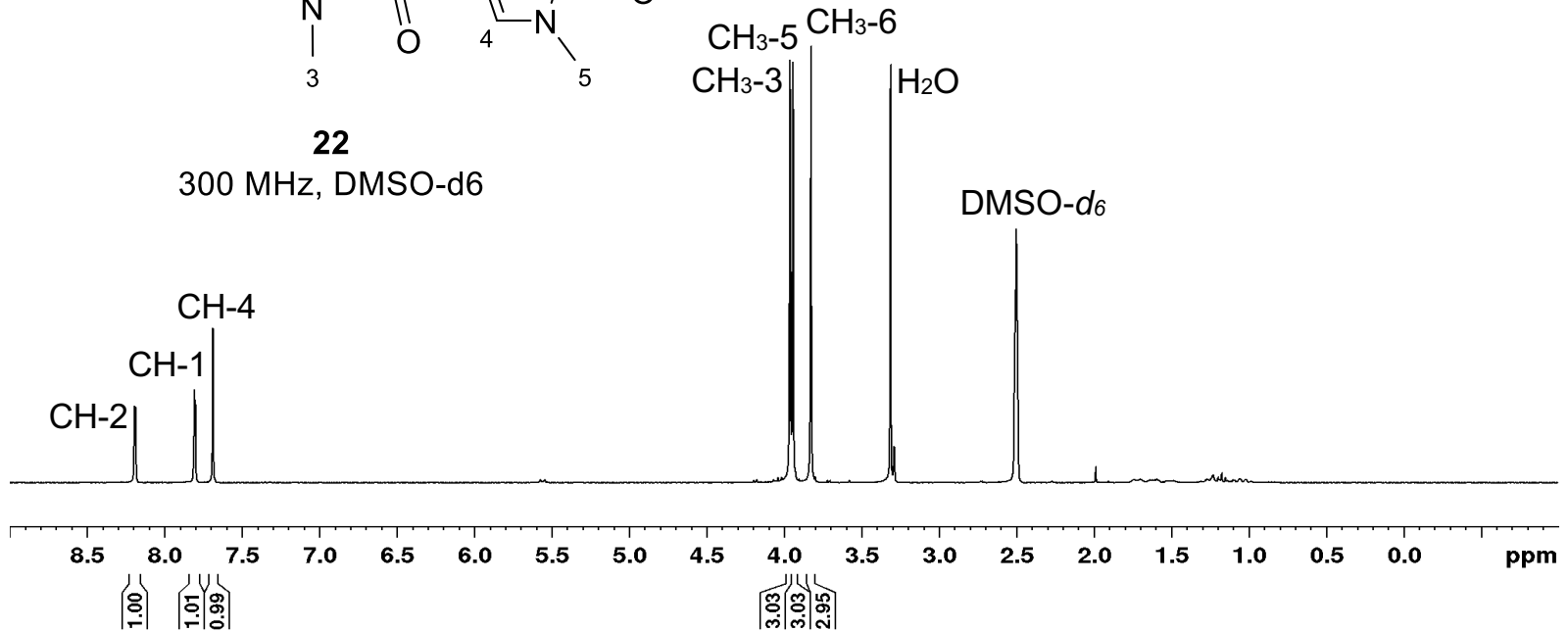

Figure S6: ${ }^{1} \mathrm{H}-\mathrm{NMR}(300 \mathrm{MHz})$ spectrum of $\mathrm{O}_{2} \mathrm{~N}-\mathrm{Py}-\mathrm{Im}-\mathrm{OMe}$ (22). 


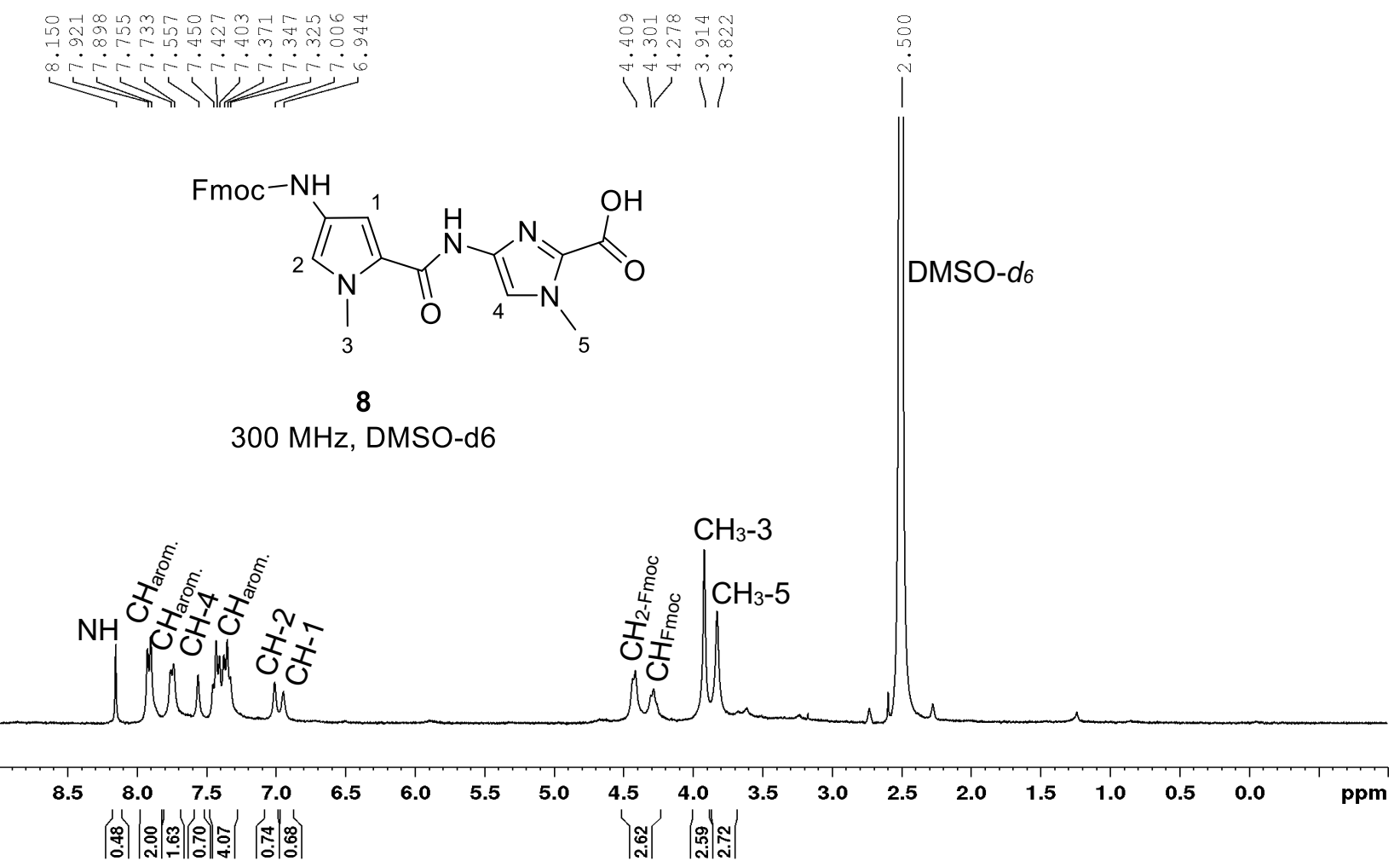

Figure S7: ${ }^{1} \mathrm{H}-\mathrm{NMR}(300 \mathrm{MHz})$ spectrum of Fmoc-Py-Im-OH (8).

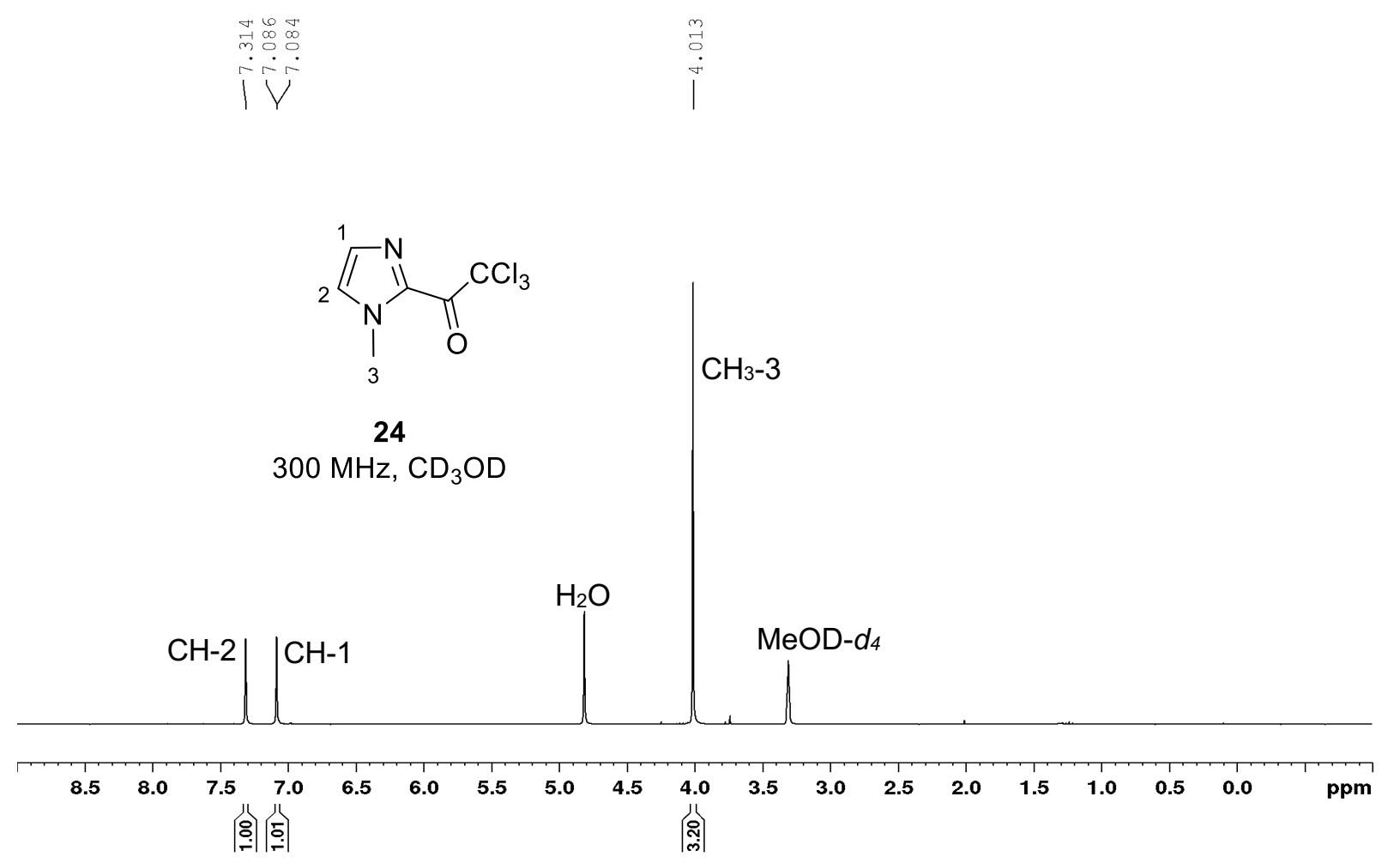

Figure S8: ${ }^{1} \mathrm{H}-\mathrm{NMR}(300 \mathrm{MHz})$ spectrum of $\mathrm{Im}-\mathrm{CCl}_{3}$ (24). 


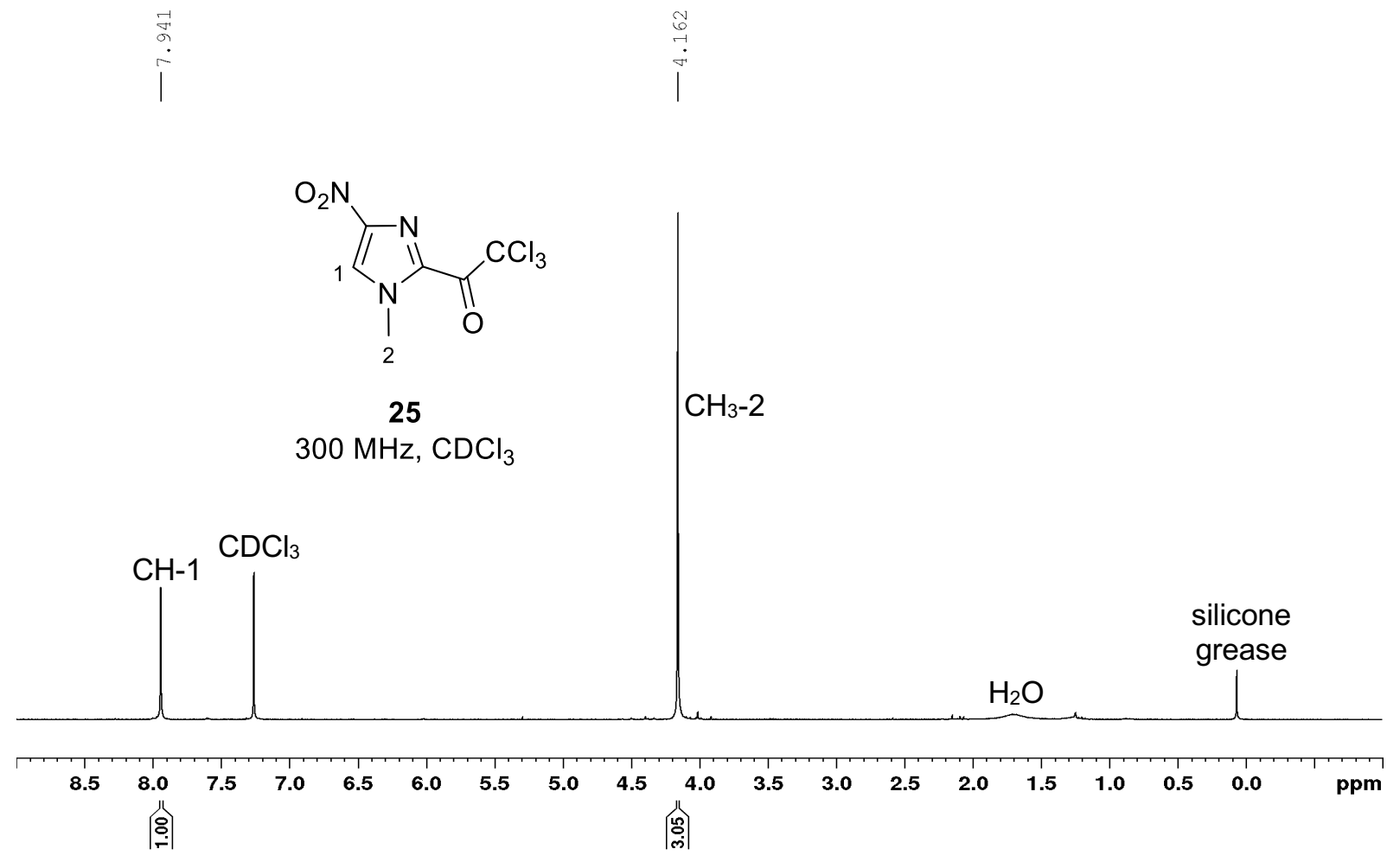

Figure S9: ${ }^{1} \mathrm{H}-\mathrm{NMR}(300 \mathrm{MHz})$ spectrum of $\mathrm{O}_{2} \mathrm{~N}-\mathrm{Im}_{-} \mathrm{CCl}_{3}$ (25).
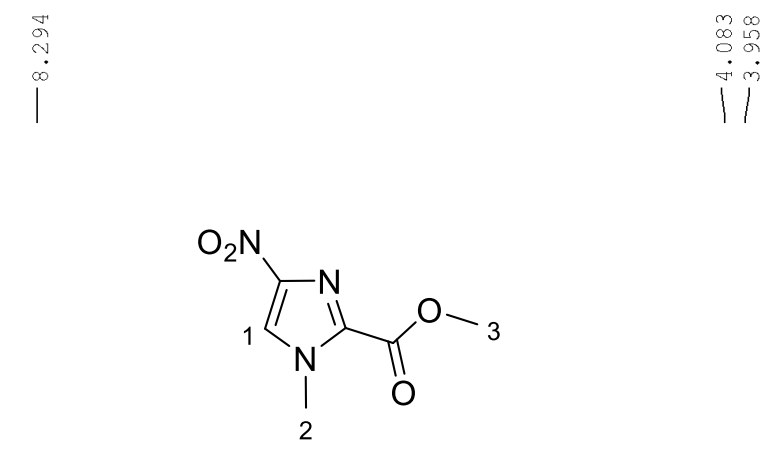

12

$300 \mathrm{MHz}, \mathrm{CD}_{3} \mathrm{OD}$

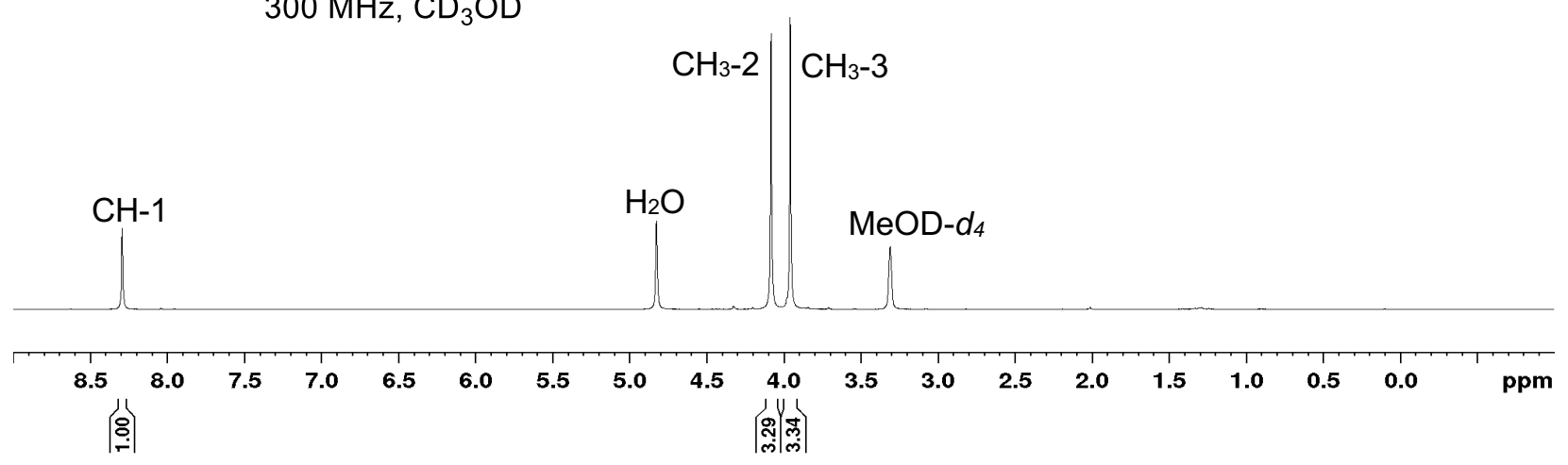

Figure S10: ${ }^{1} \mathrm{H}-\mathrm{NMR}(300 \mathrm{MHz})$ spectrum of $\mathrm{O}_{2} \mathrm{~N}-\mathrm{Im}-\mathrm{OMe}(\mathbf{1 2})$. 


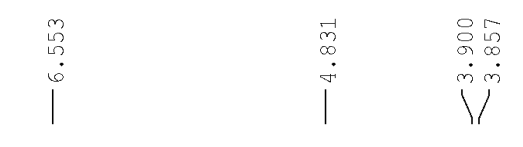<smiles>[2H]n1[14cH]c(N)nc1C(=O)OC</smiles>

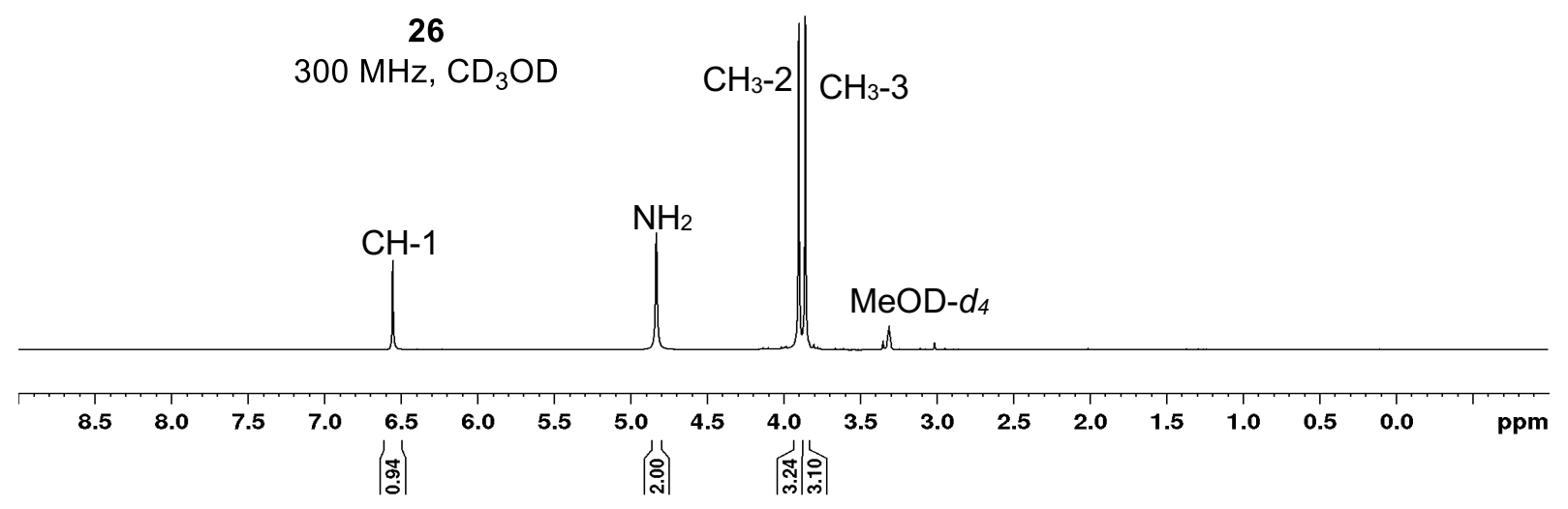

Figure S11: ${ }^{1} \mathrm{H}-\mathrm{NMR}(300 \mathrm{MHz})$ spectrum of $\mathrm{H}_{2} \mathrm{~N}-\mathrm{Im}-\mathrm{OMe}(\mathbf{2 6})$.

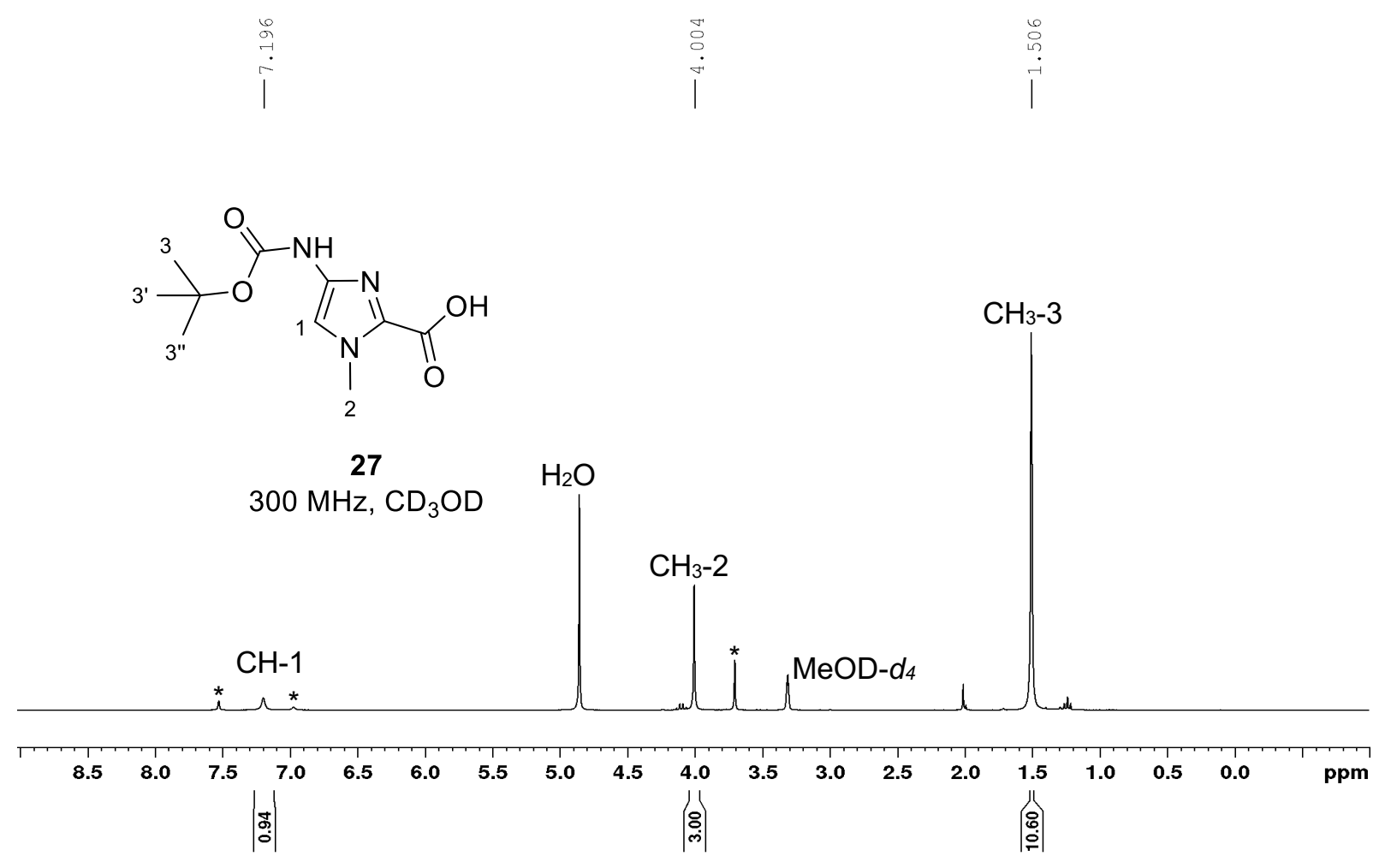

Figure S12: ${ }^{1} \mathrm{H}-\mathrm{NMR}(300 \mathrm{MHz})$ spectrum of Boc-Im-OH (27). * represents the commonly observed decarboxylated side product ( 25\% abundance). [ii,xiv] 


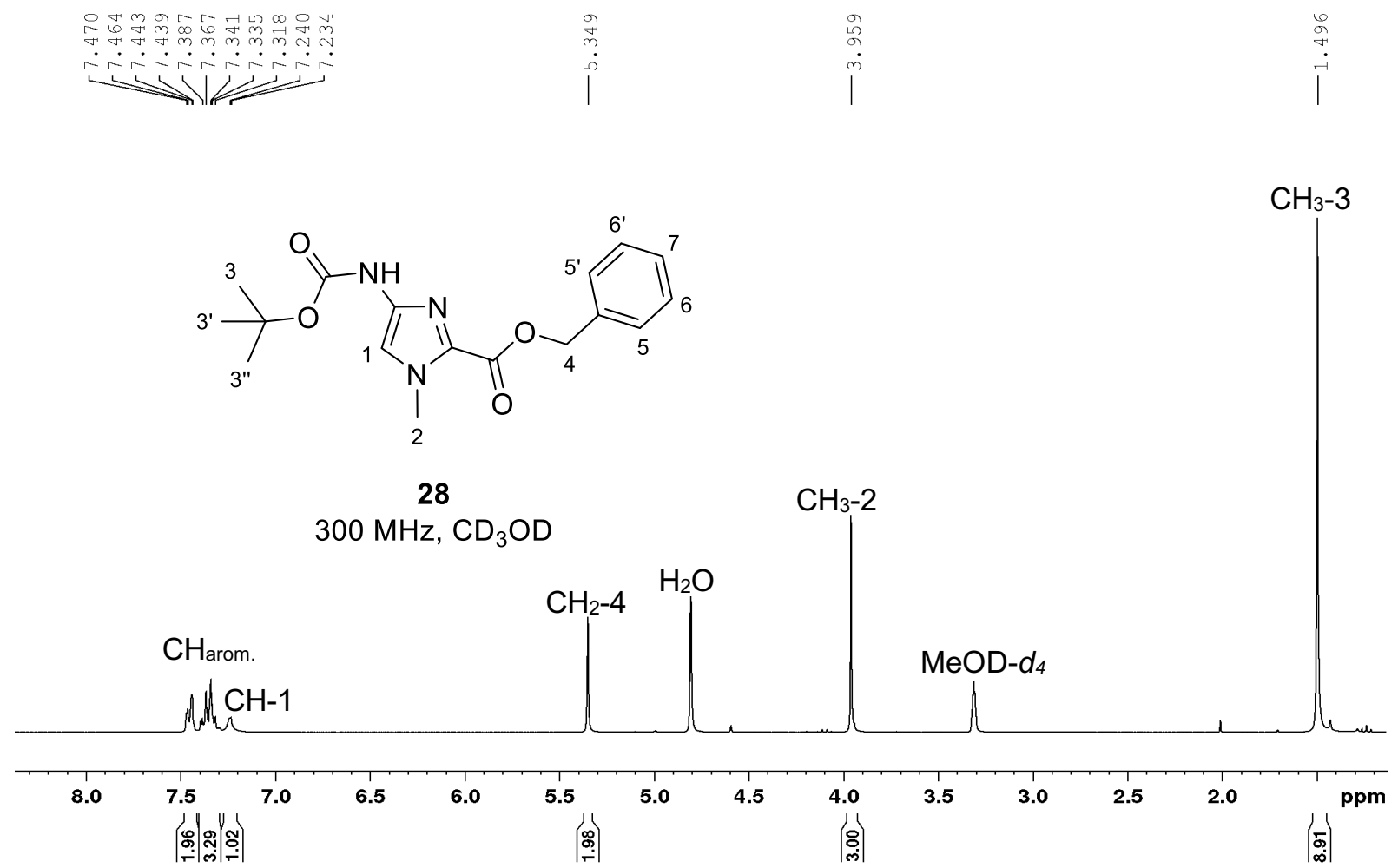

Figure S13: ${ }^{1} \mathrm{H}-\mathrm{NMR}(300 \mathrm{MHz})$ spectrum of Boc-Im-OBn (28).

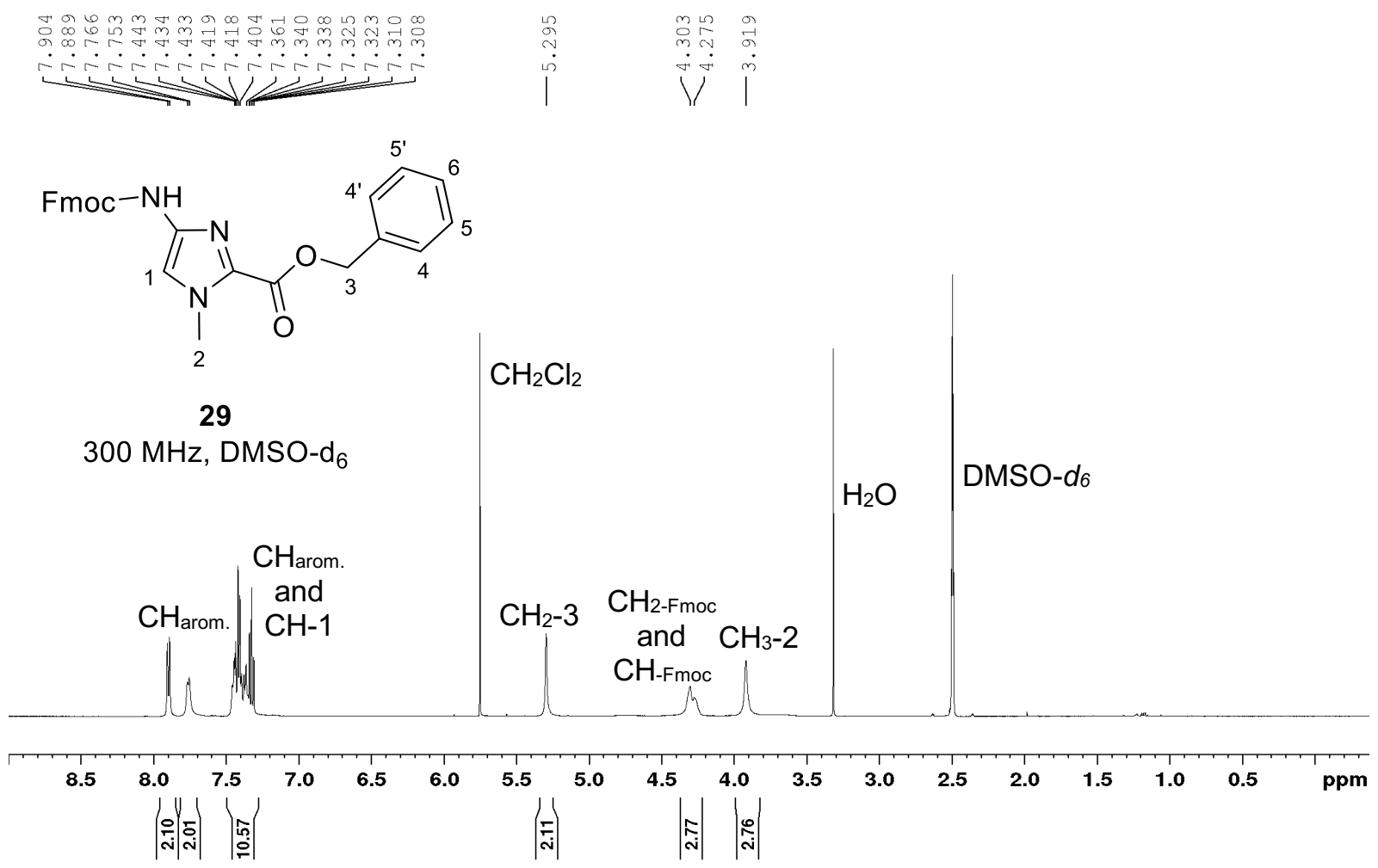

Figure S14: ${ }^{1} \mathrm{H}-\mathrm{NMR}(300 \mathrm{MHz})$ spectrum of Fmoc-Im-OBn (29). 

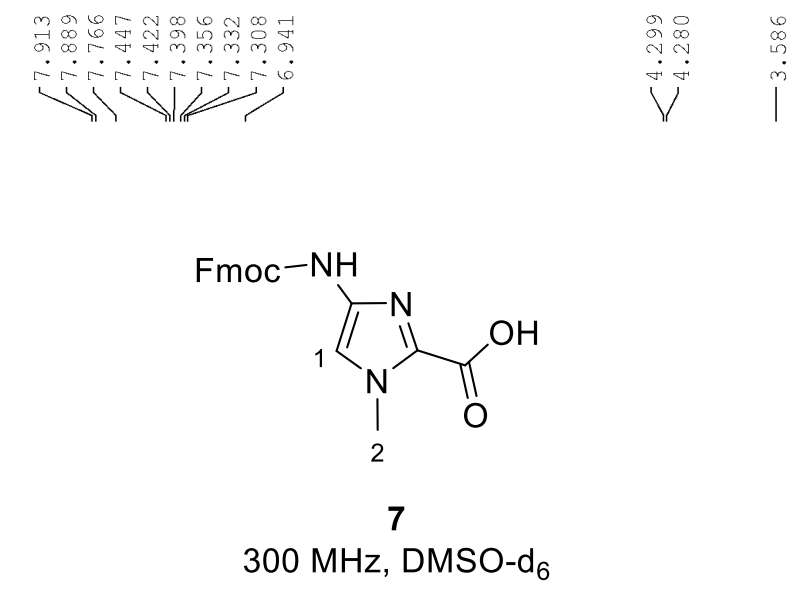

DMSO- $d_{6}$

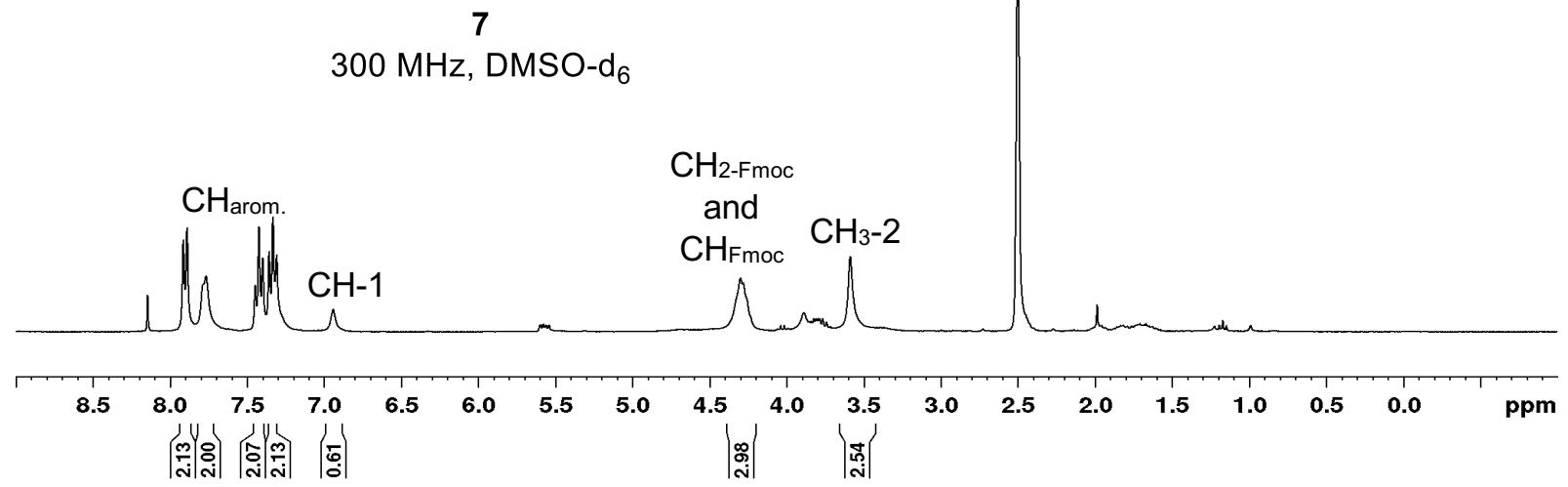

Figure S15: ${ }^{1} \mathrm{H}-\mathrm{NMR}$ (300 MHz) spectrum of Fmoc-Im-OH (7).

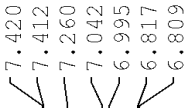

बल

411

11

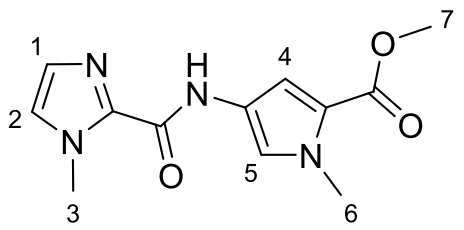

30

$300 \mathrm{MHz}, \mathrm{CDCl}_{3}$
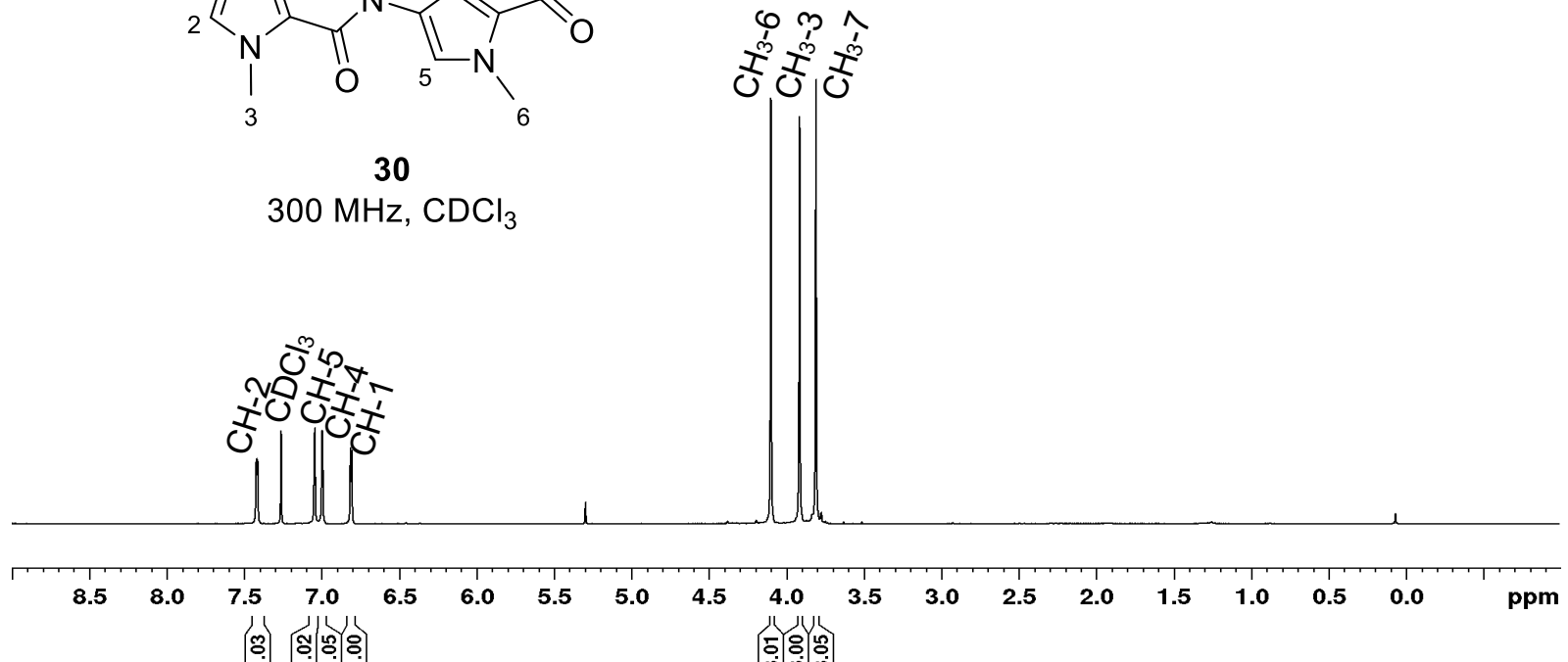

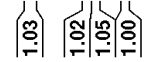

Figure S16: ${ }^{1} \mathrm{H}-\mathrm{NMR}(300 \mathrm{MHz})$ spectrum of Im-Py-OMe (30). 
$\mid$

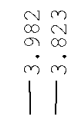<smiles>[2H]n1cc(NC(=O)c2nccn2C)cc1C(=O)O</smiles>

31

$300 \mathrm{MHz}, \mathrm{DMSO}-\mathrm{d}_{6}$

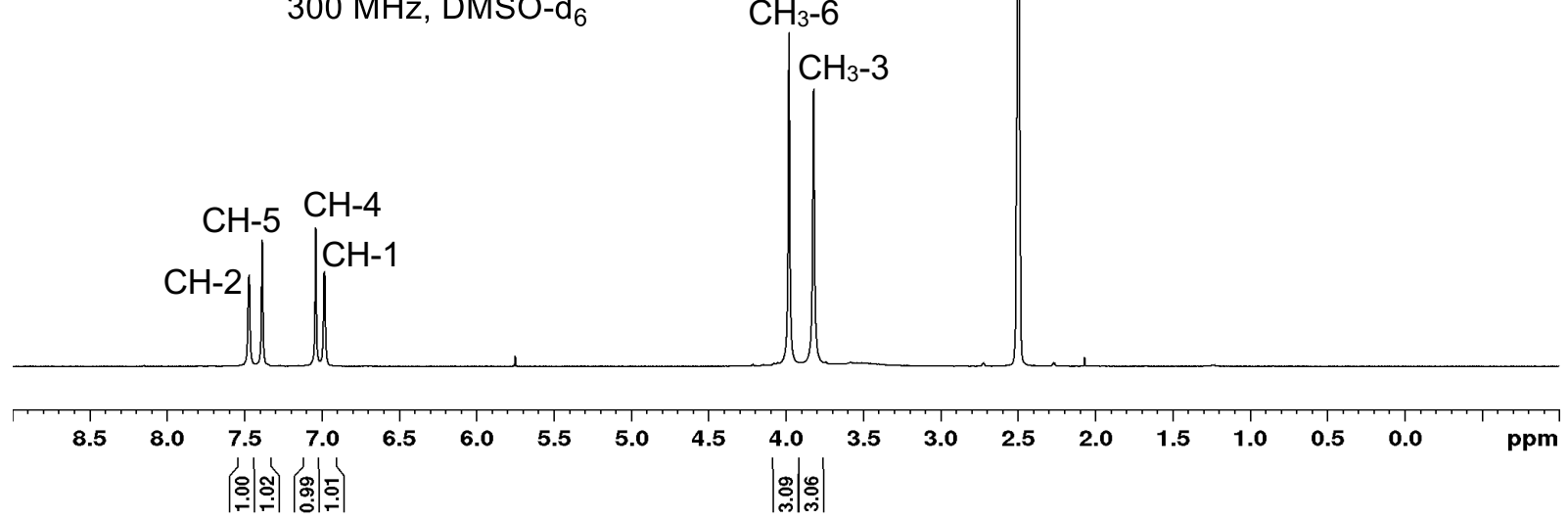

Figure S17: ${ }^{1} \mathrm{H}-\mathrm{NMR}(300 \mathrm{MHz})$ spectrum of Im-Py-OH (31).

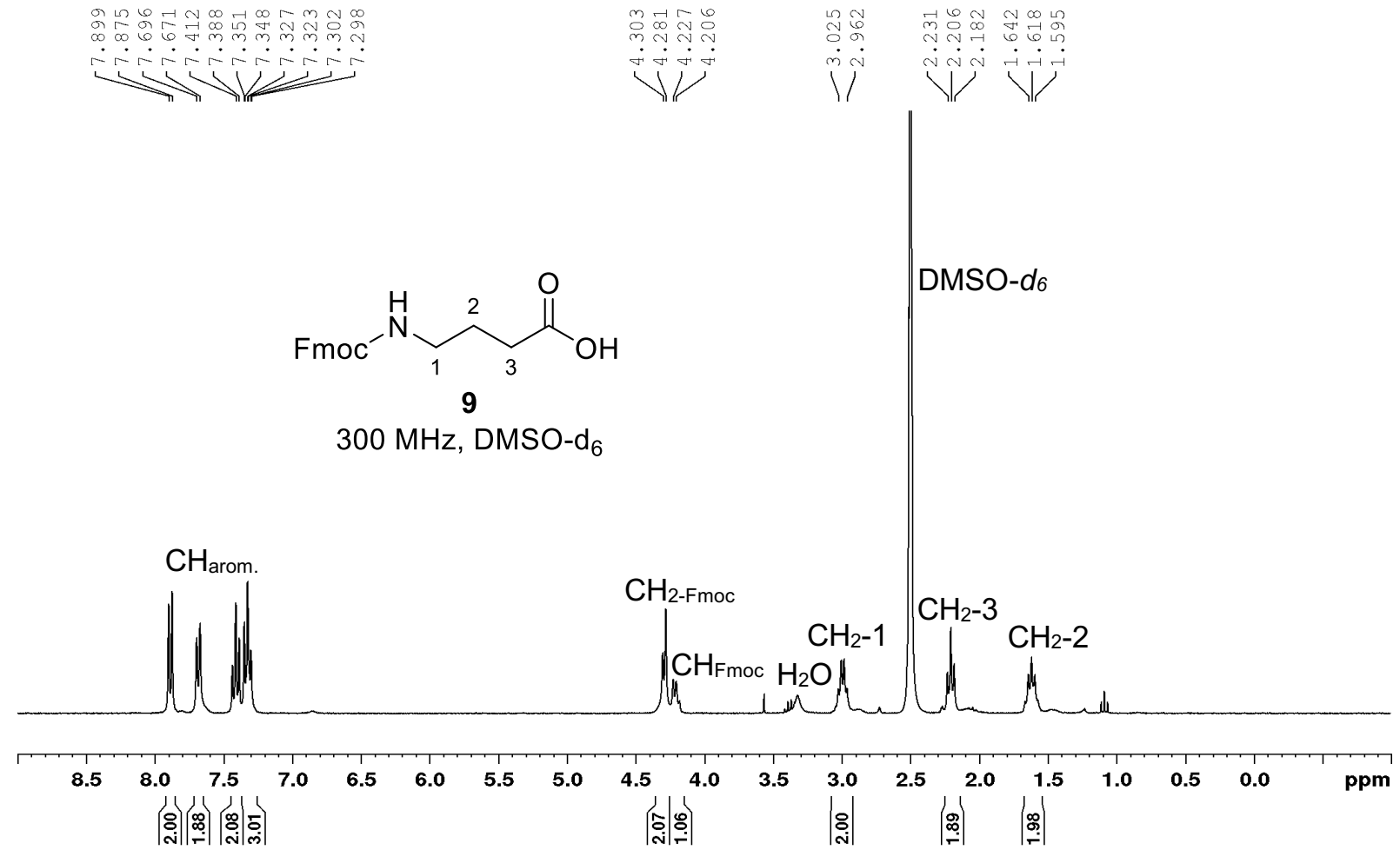

Figure S18: ${ }^{1} \mathrm{H}-\mathrm{NMR}(300 \mathrm{MHz})$ spectrum of Fmoc- $\gamma-\mathrm{OH}(\mathbf{9})$. 


\section{NMR and IR spectra of new compounds}
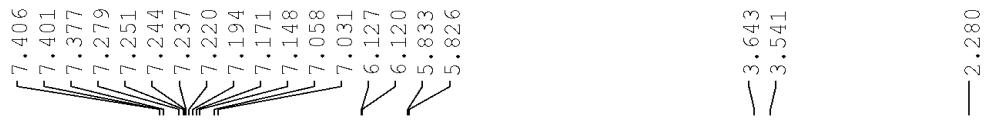<smiles>[M]CNc1ccc(C(=O)OC)n1C</smiles>

11

$300 \mathrm{MHz}, \mathrm{CD}_{3} \mathrm{OD}$

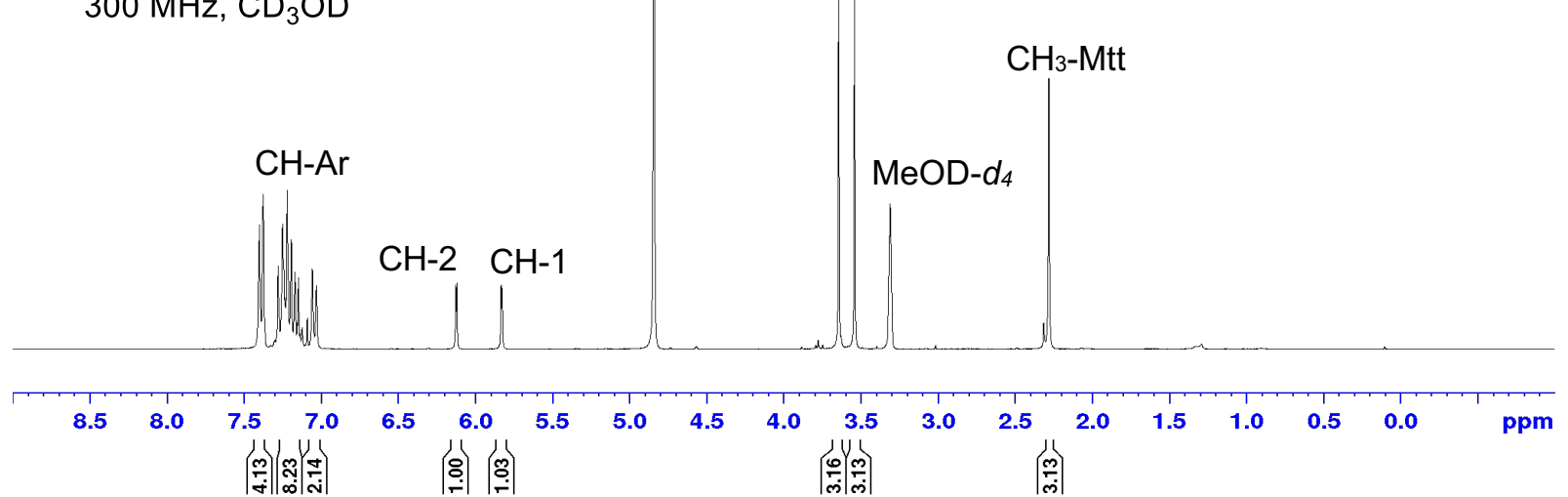

Figure S19: ${ }^{1} \mathrm{H}-\mathrm{NMR}(300 \mathrm{MHz})$ spectrum of Mtt-Py-OMe (11).

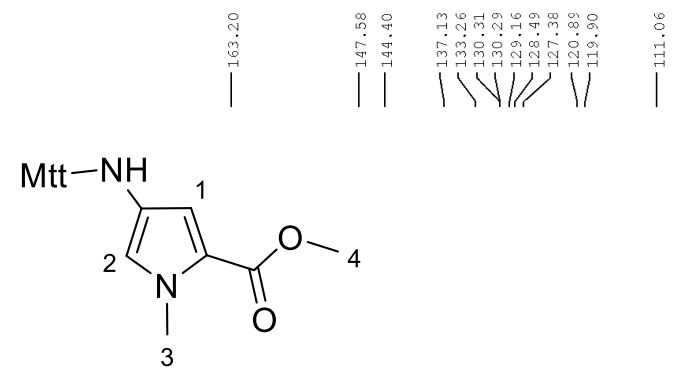

11

$75 \mathrm{MHz}, \mathrm{CD}_{3} \mathrm{OD}$

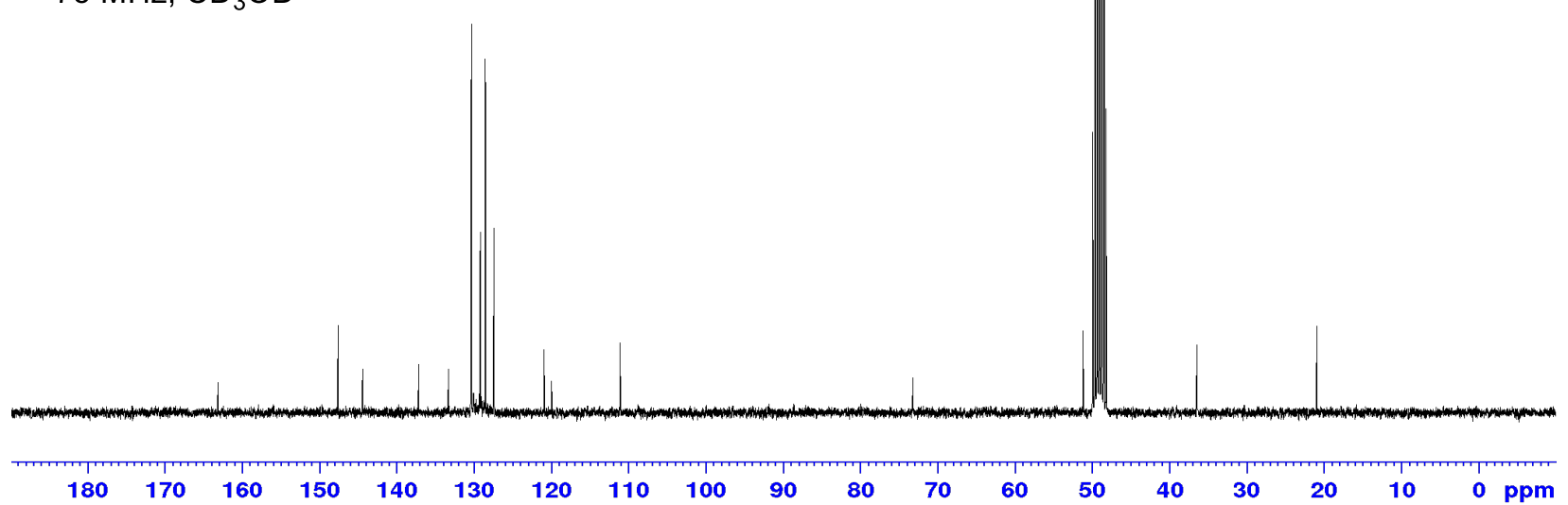

Figure S20: ${ }^{13} \mathrm{C}-\mathrm{NMR}$ (75 MHz) spectrum of Mtt-Py-OMe (11). 


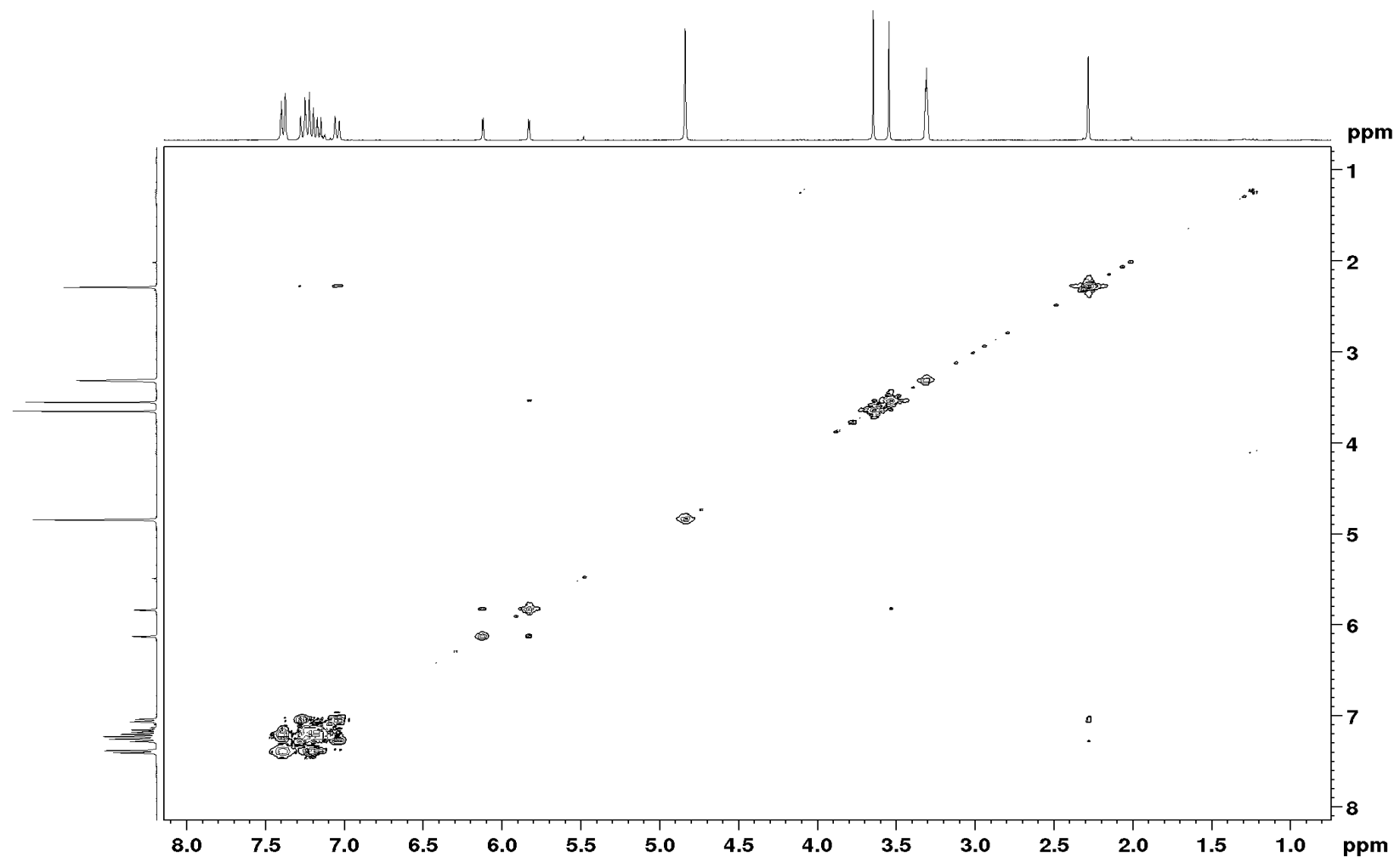

Figure S21: COSY-NMR (300 MHz) spectrum of Mtt-Py-OMe (11).

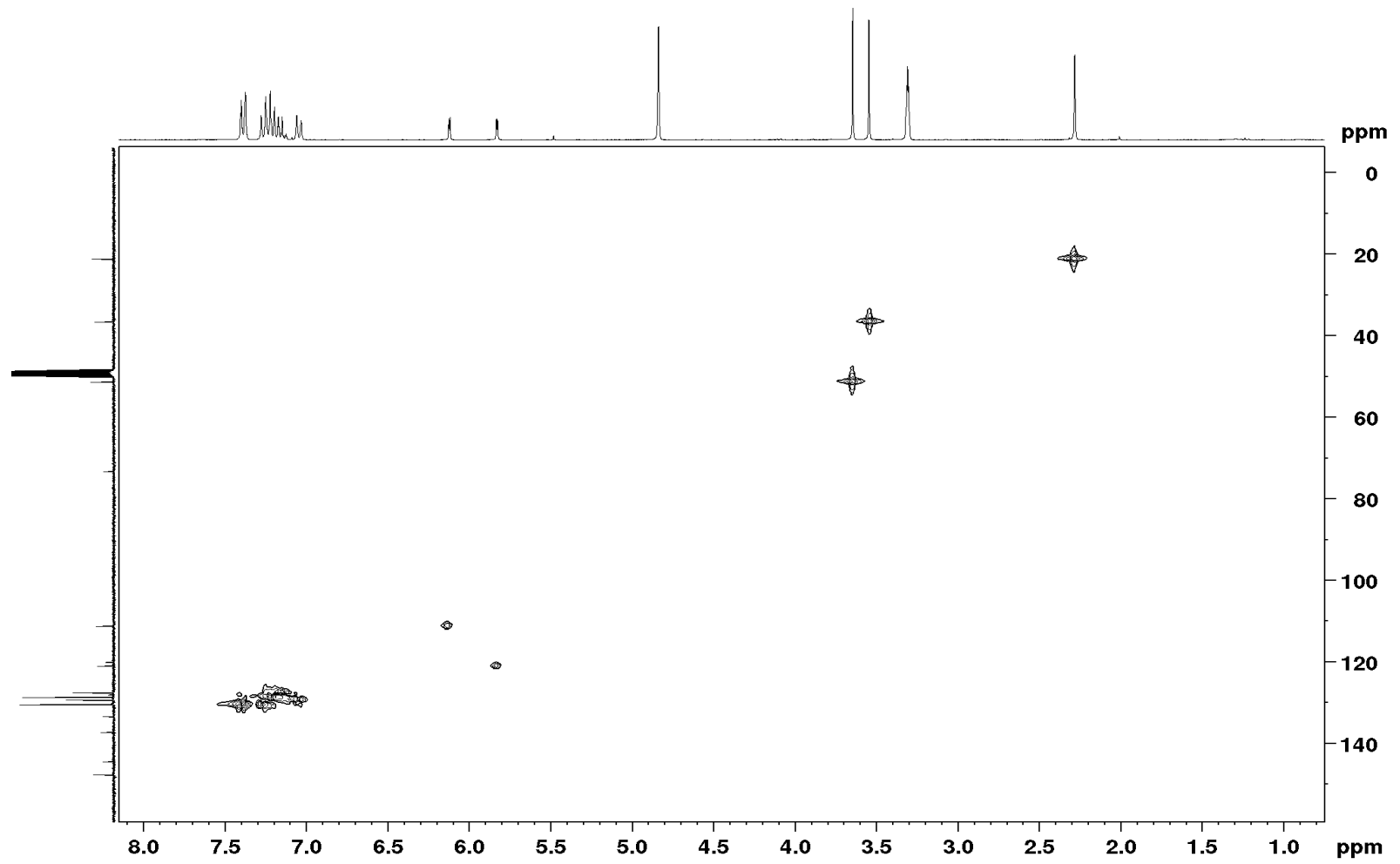

Figure S22: HSQC-NMR (300 MHz, 75 MHz) spectrum of Mtt-Py-OMe (11). 


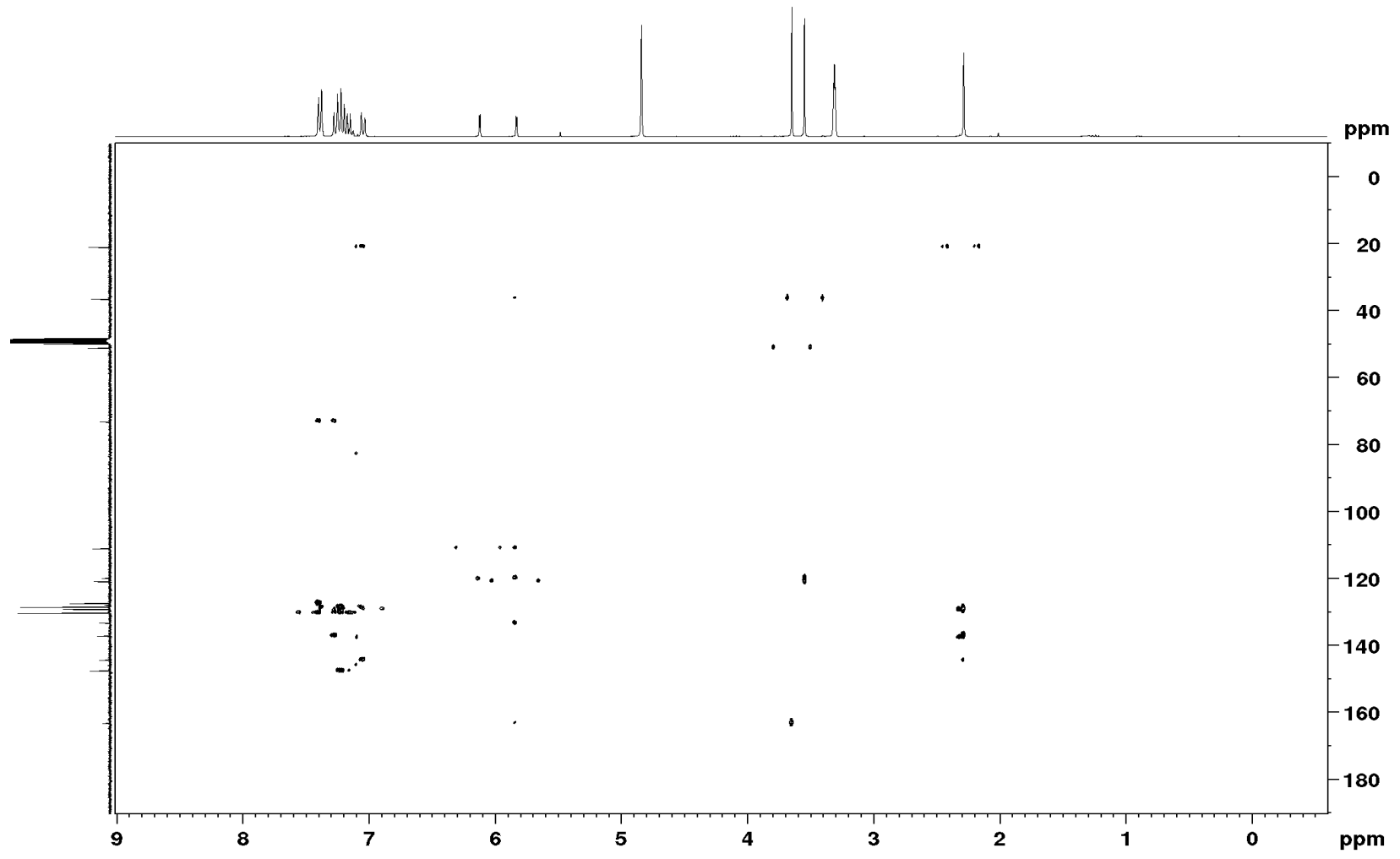

Figure S23: HMBC-NMR (500 MHz, $125 \mathrm{MHz}$ ) spectrum of Mtt-Py-OMe (11).

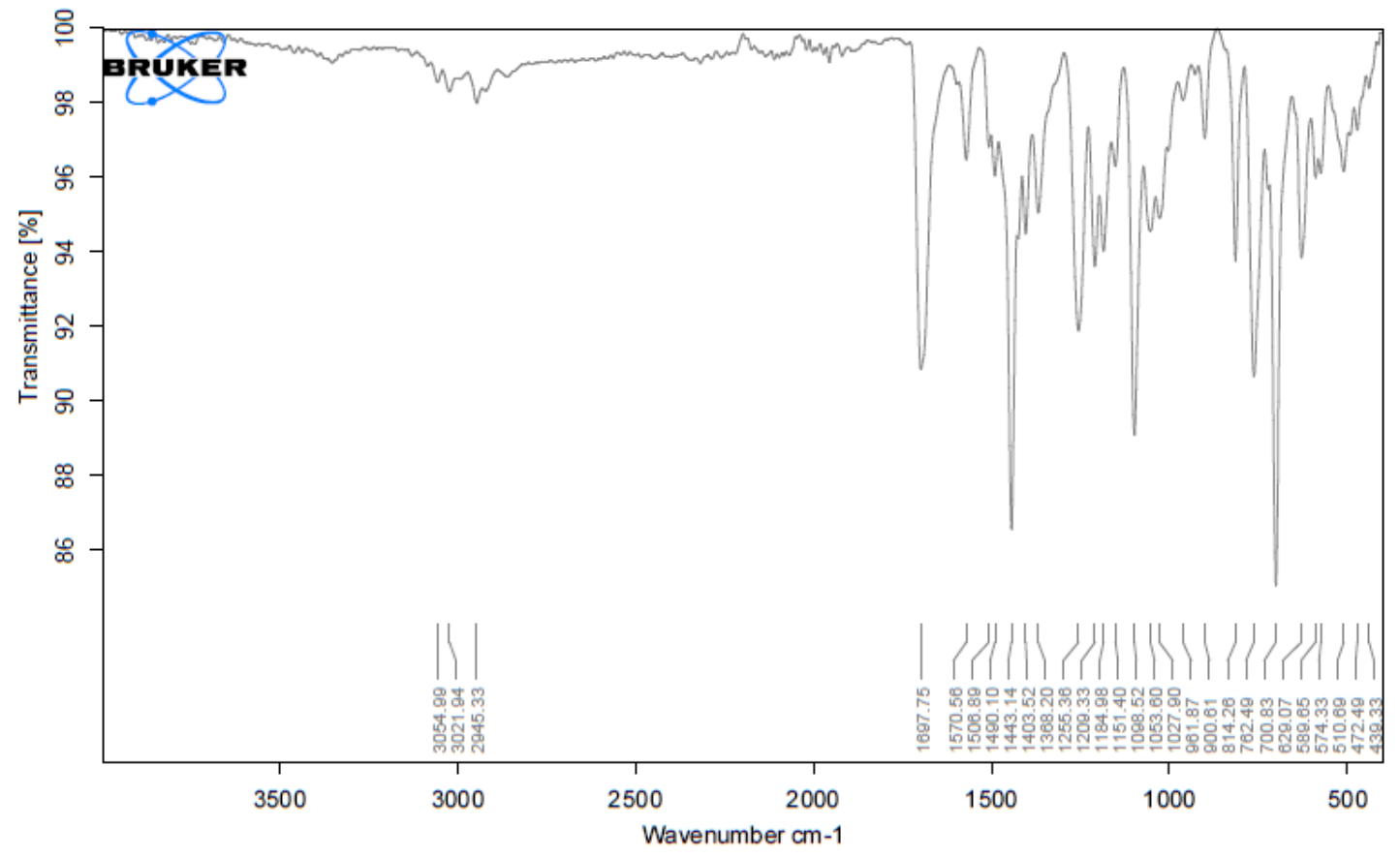

Figure S24: IR spectrum of Mtt-Py-OMe (11). 

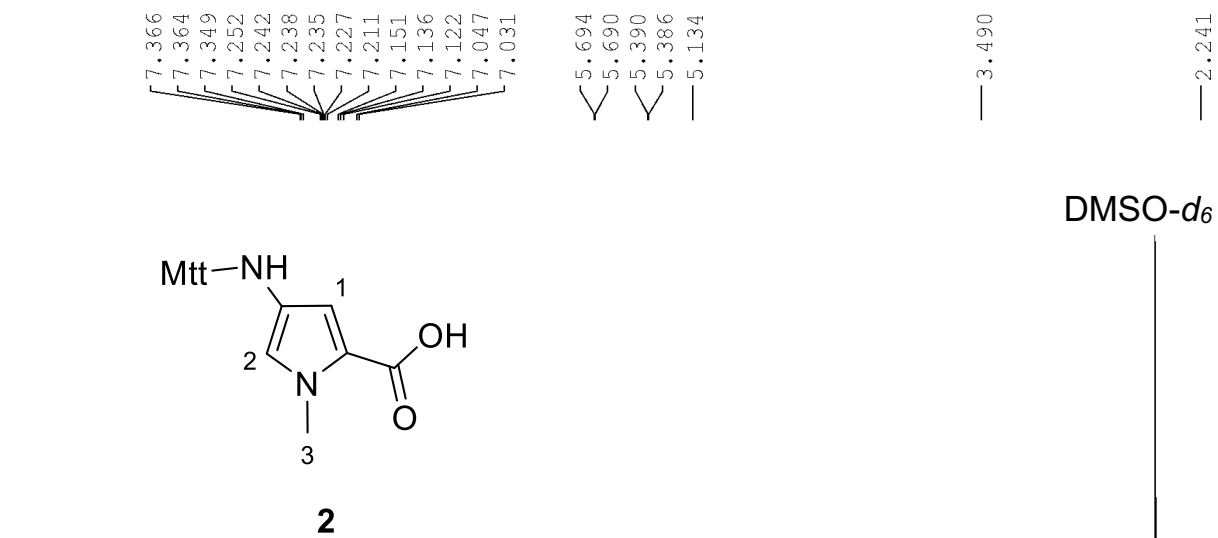

DMSO-d6

$300 \mathrm{MHz}$, DMSO-d6

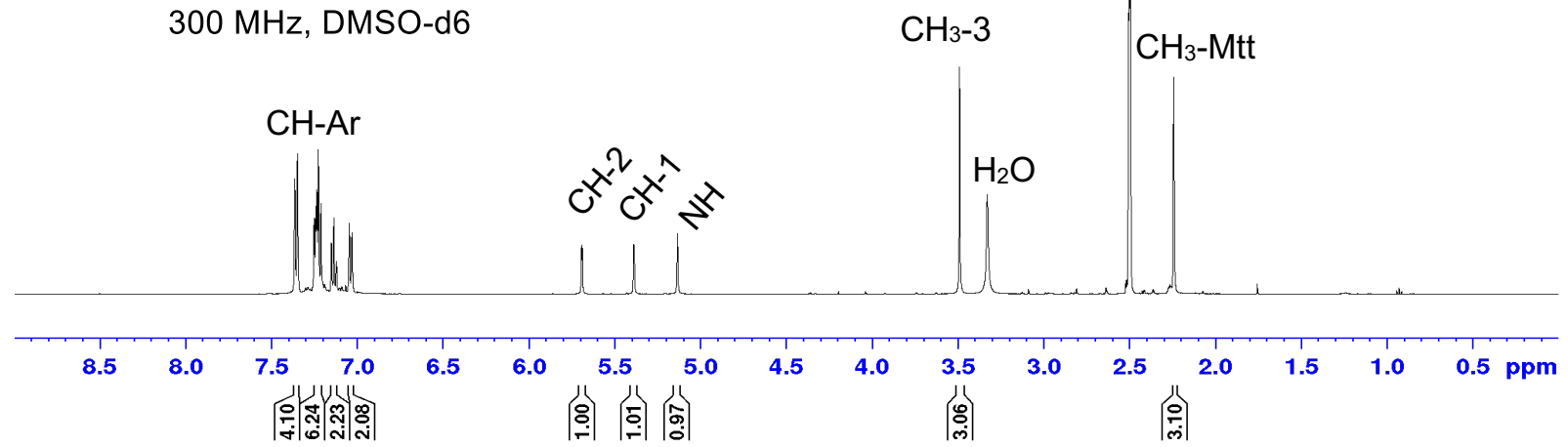

Figure S25: ${ }^{1} \mathrm{H}-\mathrm{NMR}(300 \mathrm{MHz})$ spectrum of Mtt-Py-OH (2).

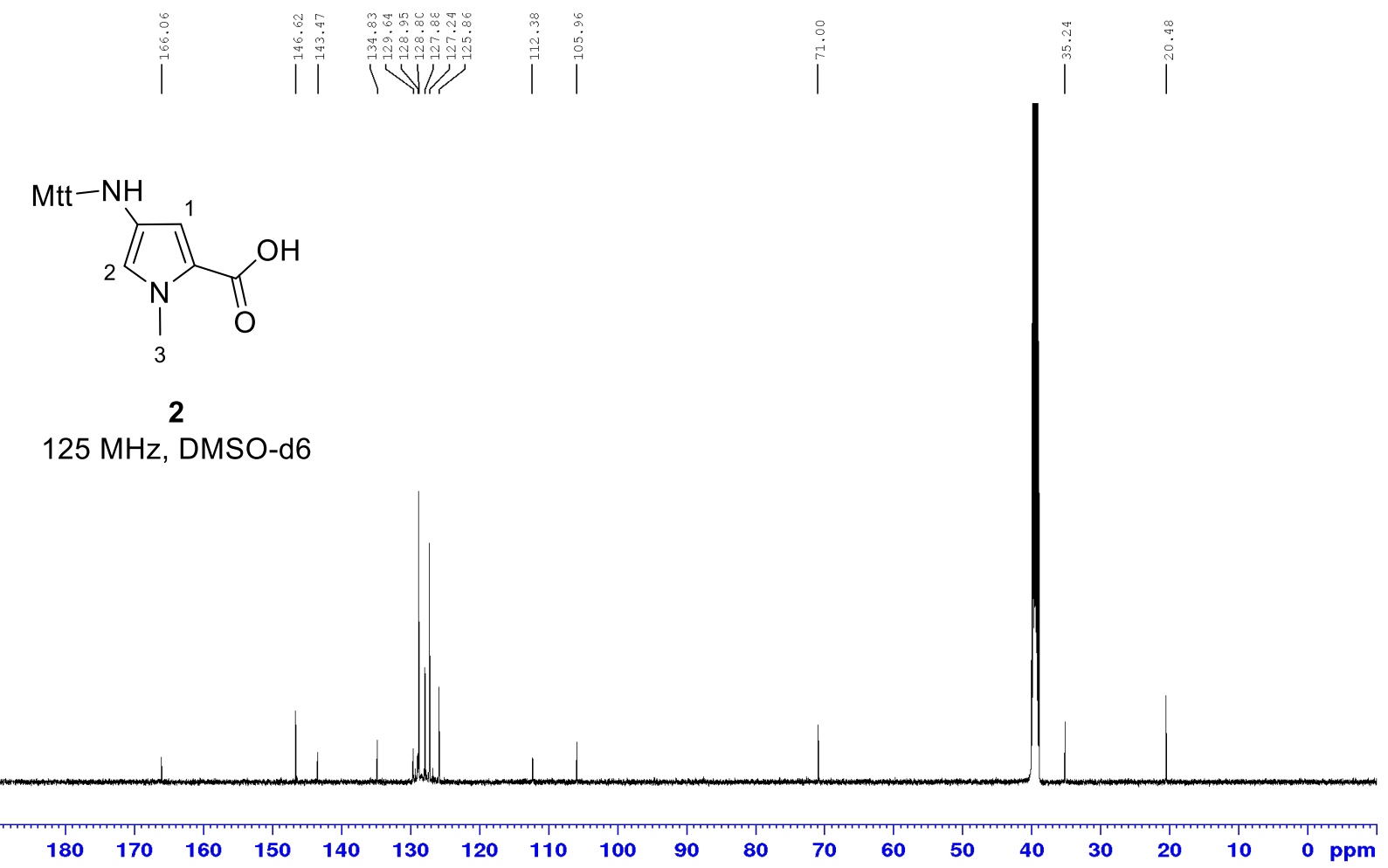

Figure S26: ${ }^{13} \mathrm{C}-\mathrm{NMR}(125 \mathrm{MHz})$ spectrum of Mtt-Py-OH (2). 


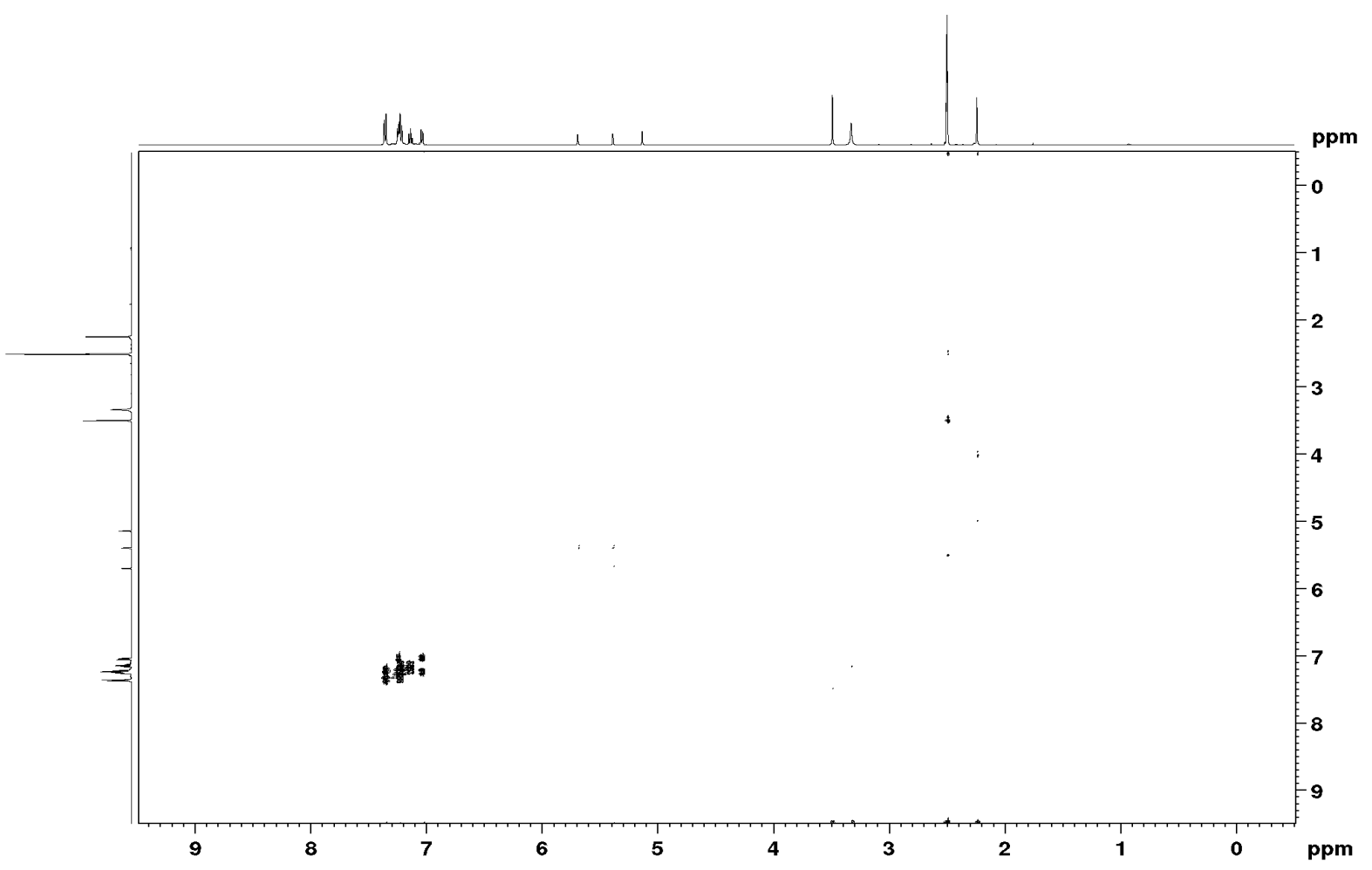

Figure S27: COSY-NMR (500 MHz) spectrum of Mtt-Py-OH (2).

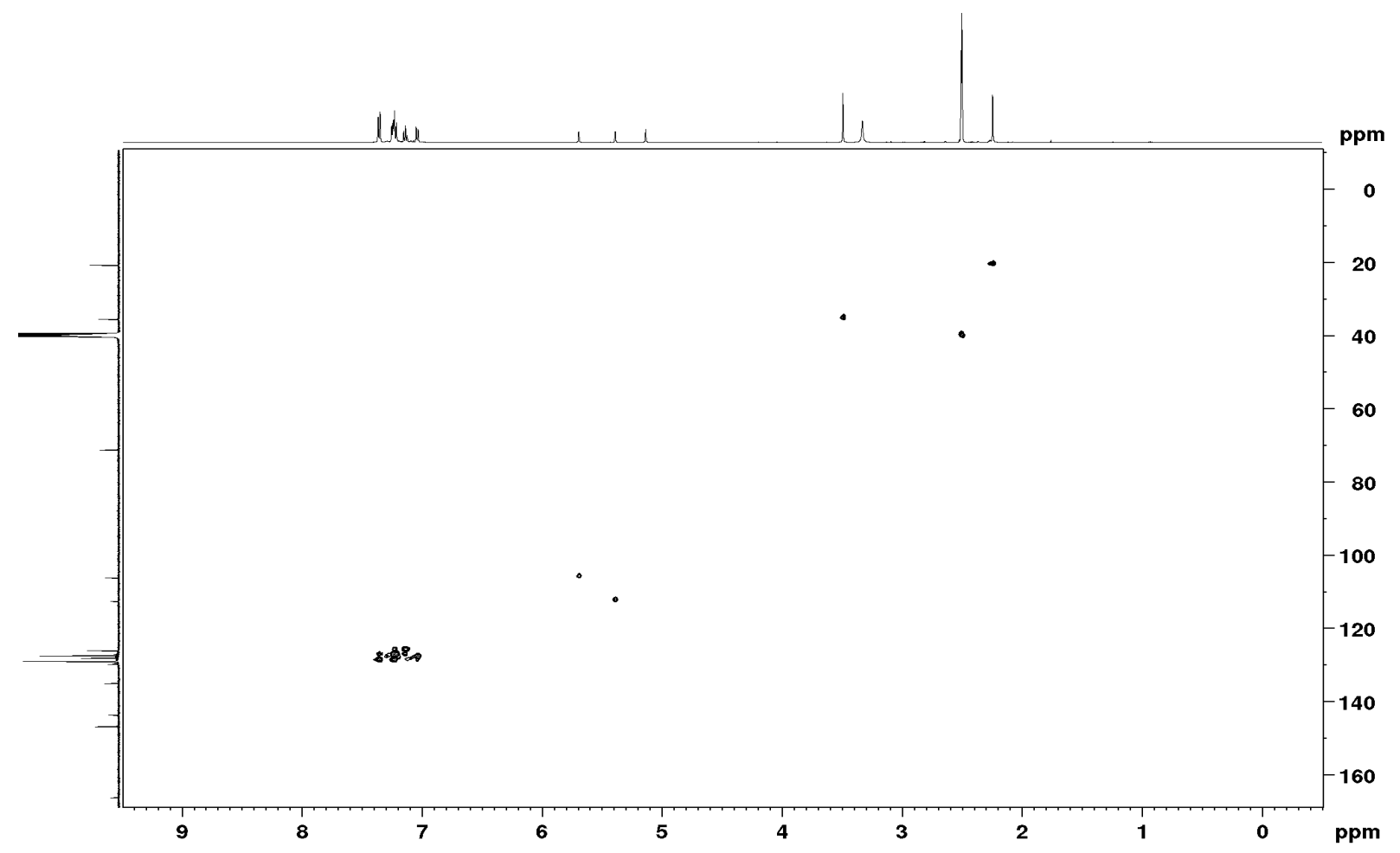

Figure S28: HSQC-NMR (500 MHz, $125 \mathrm{MHz}$ ) spectrum of Mtt-Py-OH (2). 


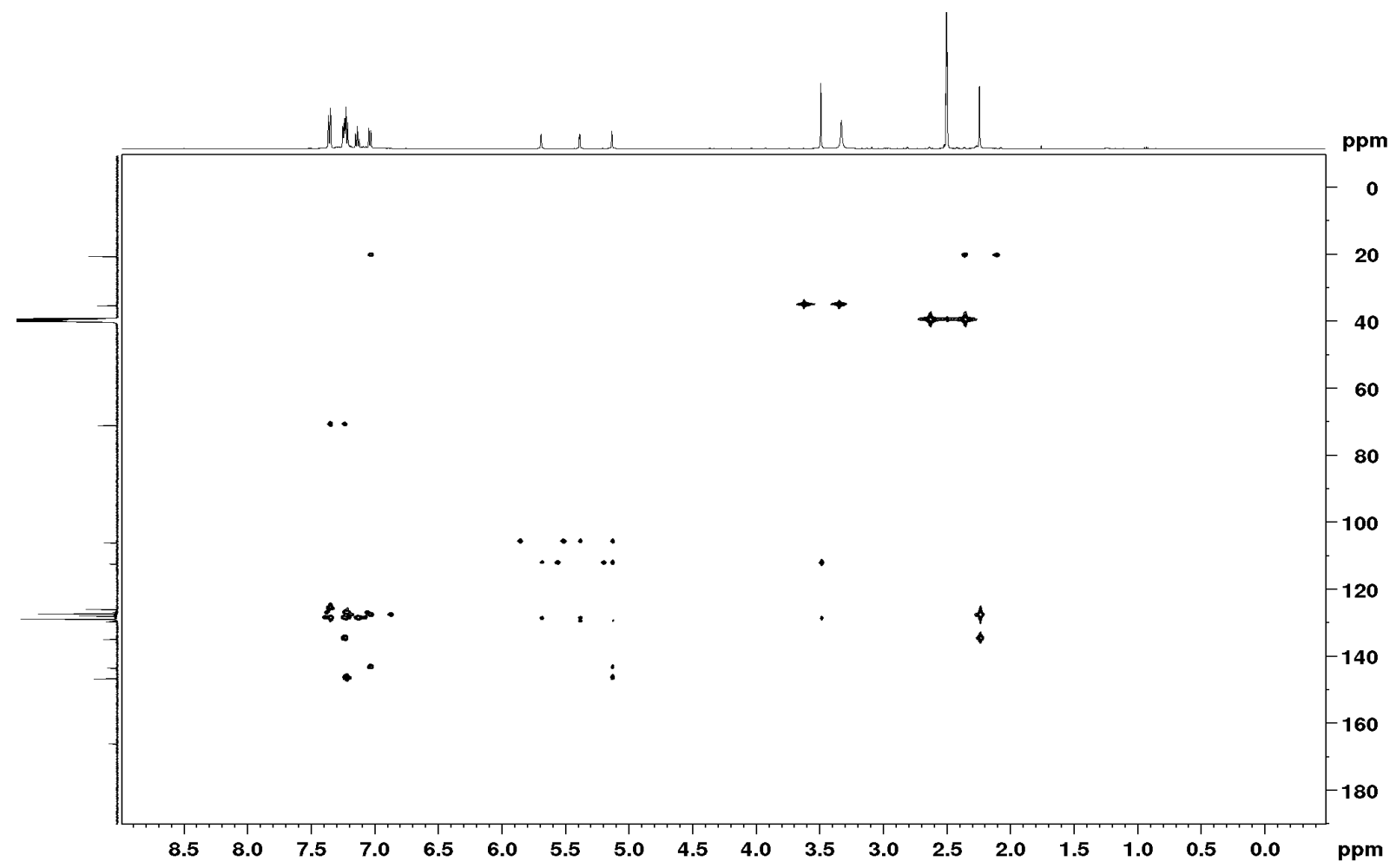

Figure S29: HMBC-NMR (500 MHz, $125 \mathrm{MHz}$ ) spectrum of Mtt-Py-OH (2).

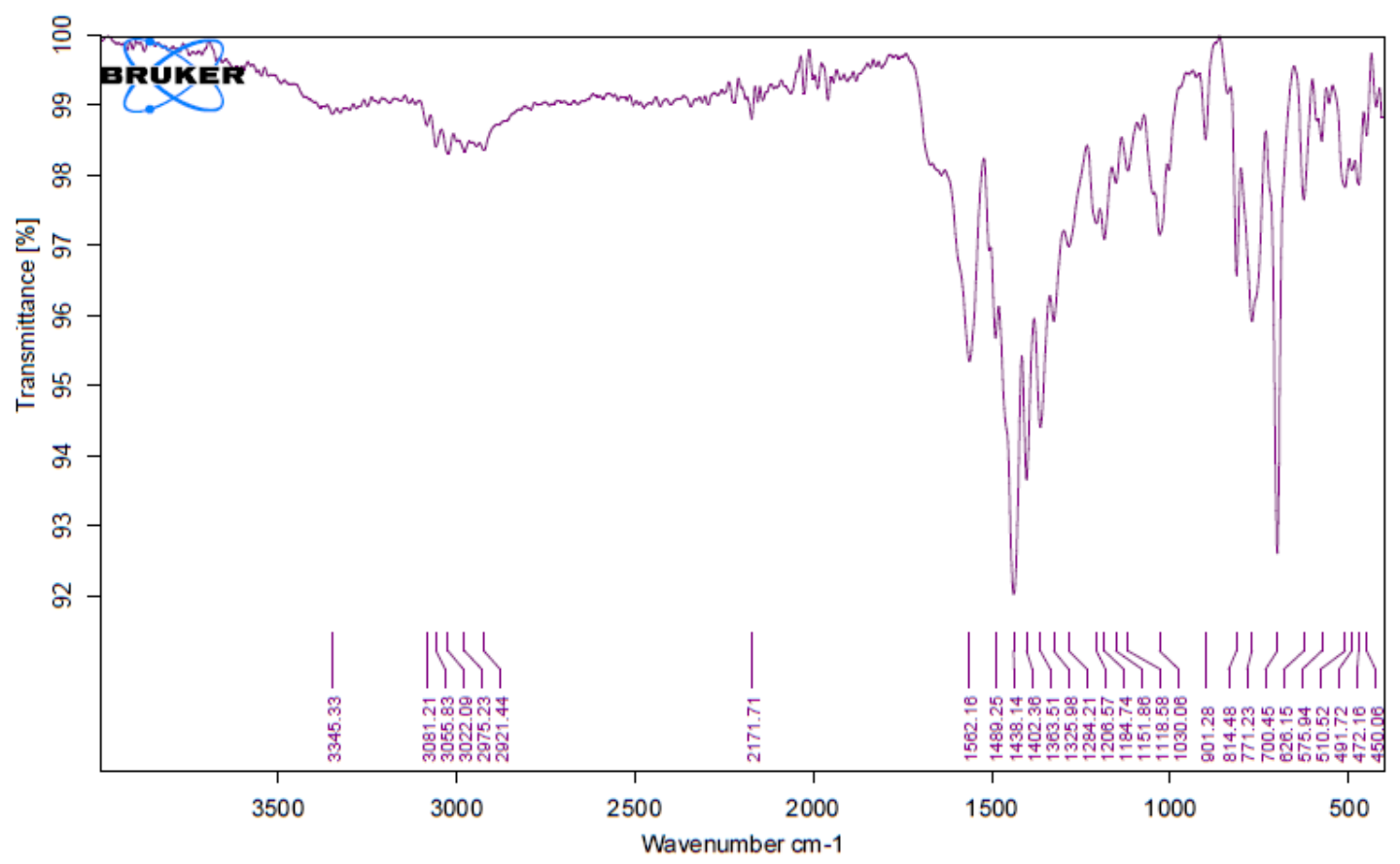

Figure S30: IR spectrum of Mtt-Py-OH (2). 


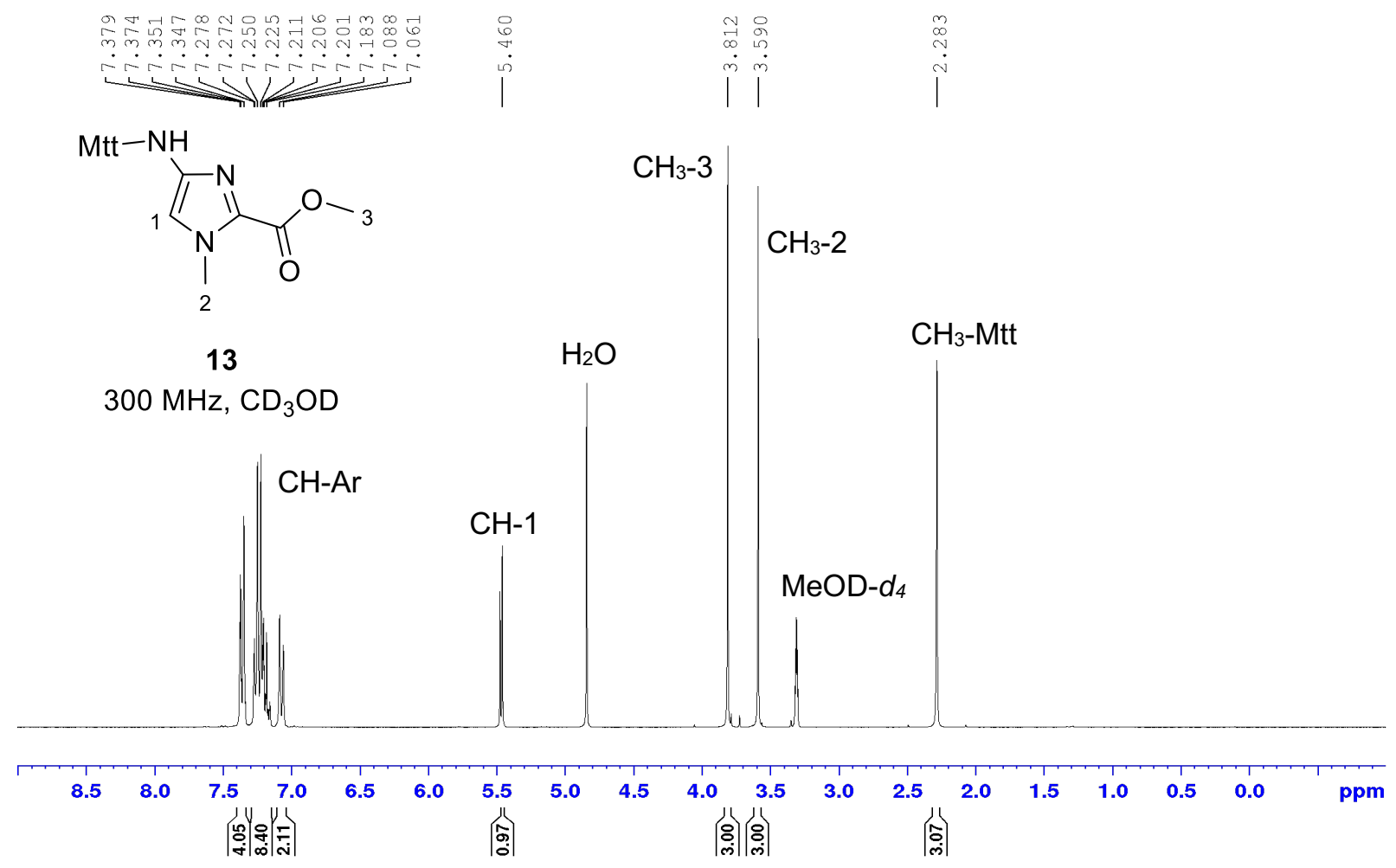

Figure S31: ${ }^{1} \mathrm{H}-\mathrm{NMR}(300 \mathrm{MHz})$ spectrum of Mtt-Im-OMe (13).

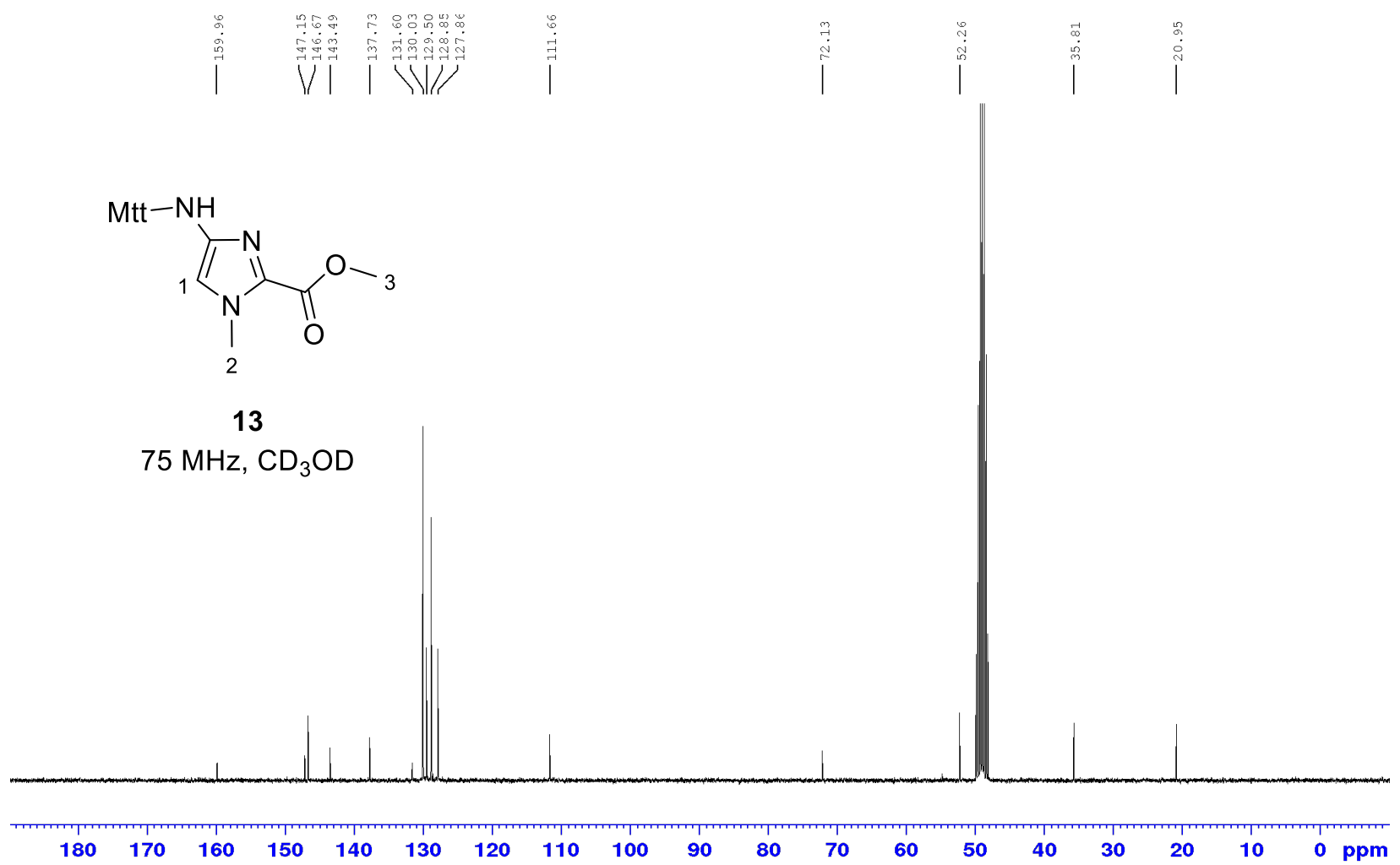

Figure S32: ${ }^{13} \mathrm{C}-\mathrm{NMR}(75 \mathrm{MHz})$ spectrum of Mtt-Im-OMe (13). 


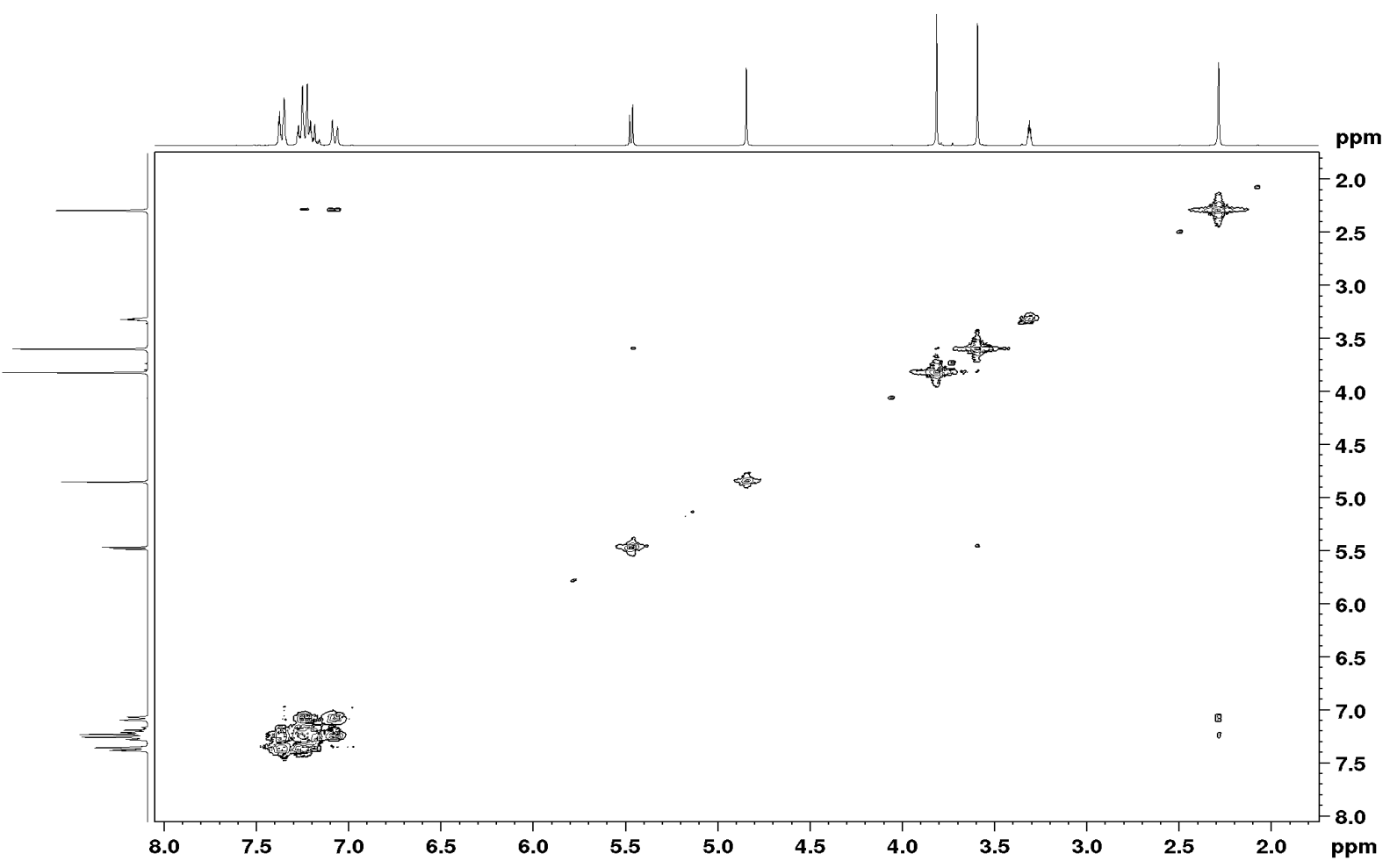

Figure S33: COSY-NMR (300 MHz) spectrum of Mtt-Im-OMe (13).

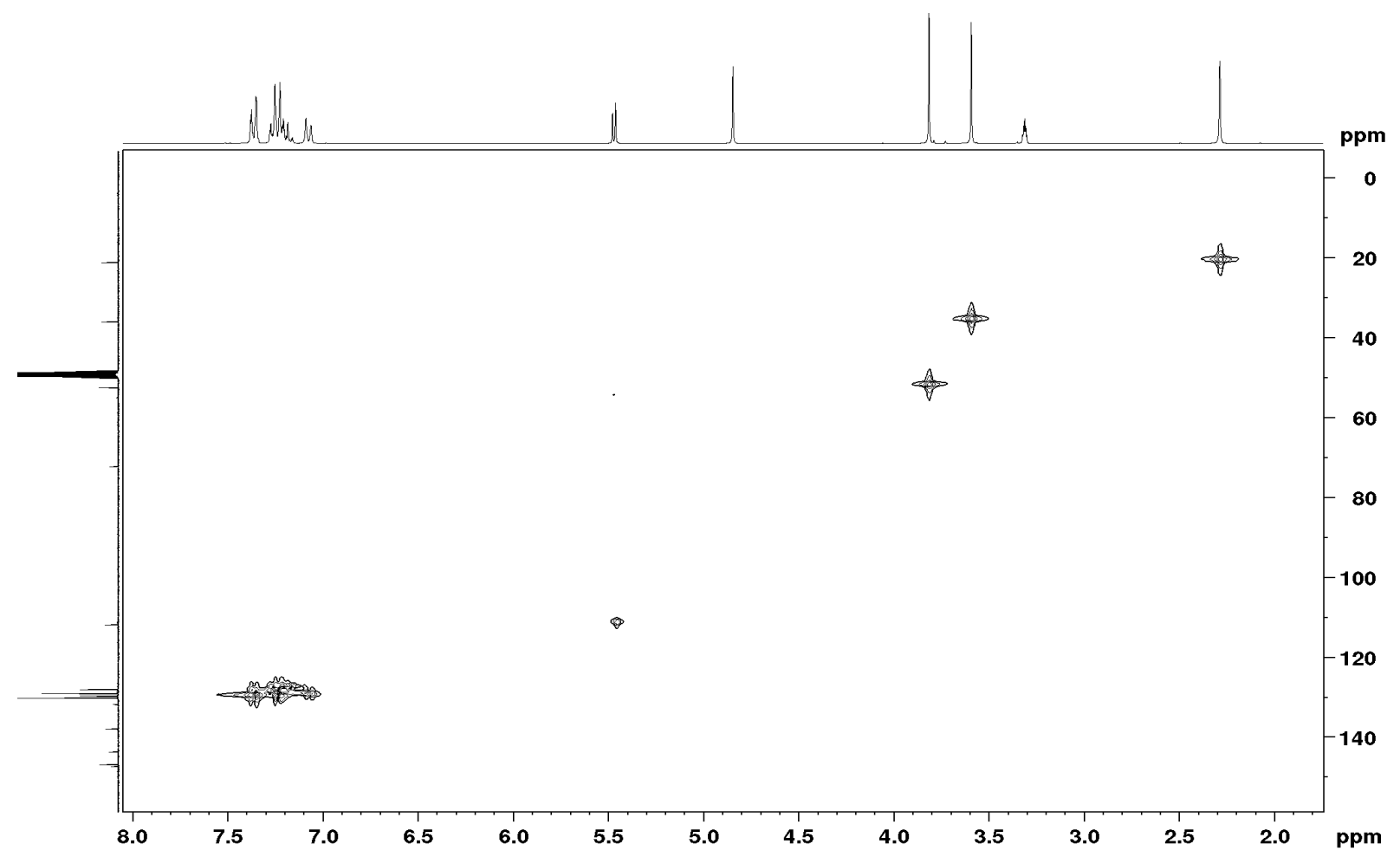

Figure S34: HSQC-NMR (300 MHz, $75 \mathrm{MHz}$ ) spectrum of Mtt-Im-OMe (13). 


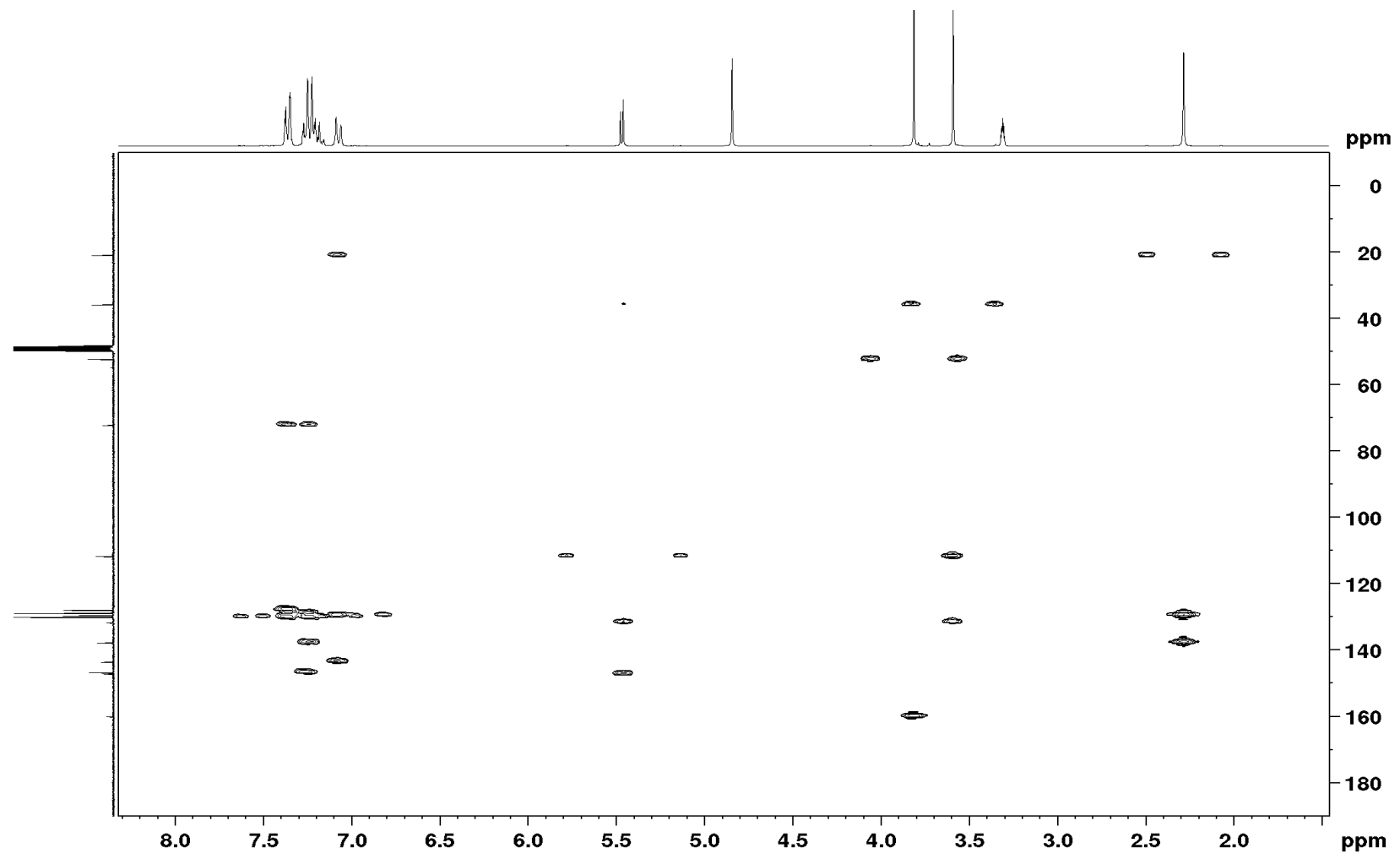

Figure S35: HMBC-NMR (500 MHz, $125 \mathrm{MHz})$ spectrum of Mtt-Im-OMe (13).

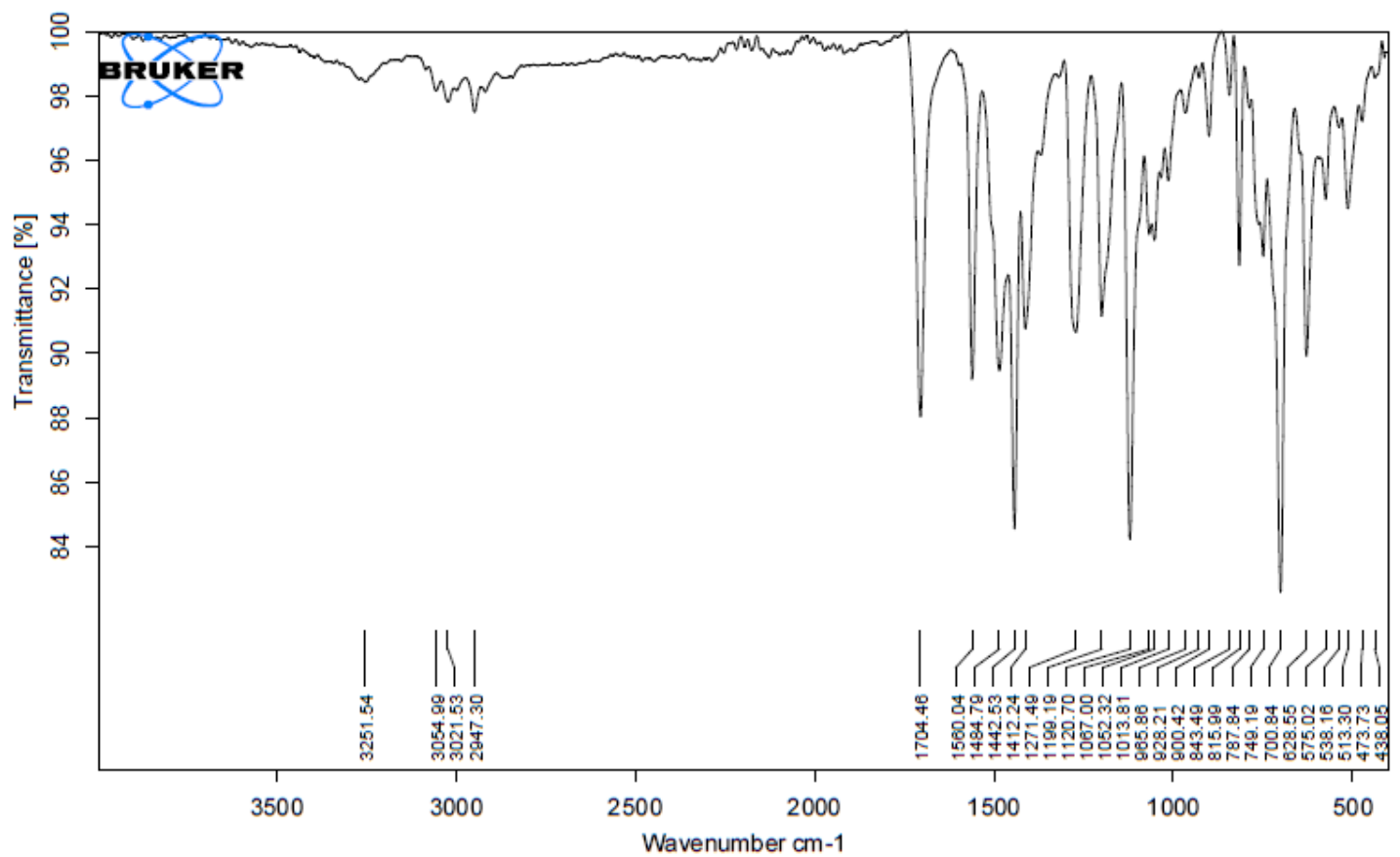

Figure S36: IR spectrum of Mtt-Im-OMe (13). 


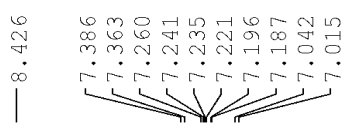

$\mathrm{Mtt}-\mathrm{NH}$
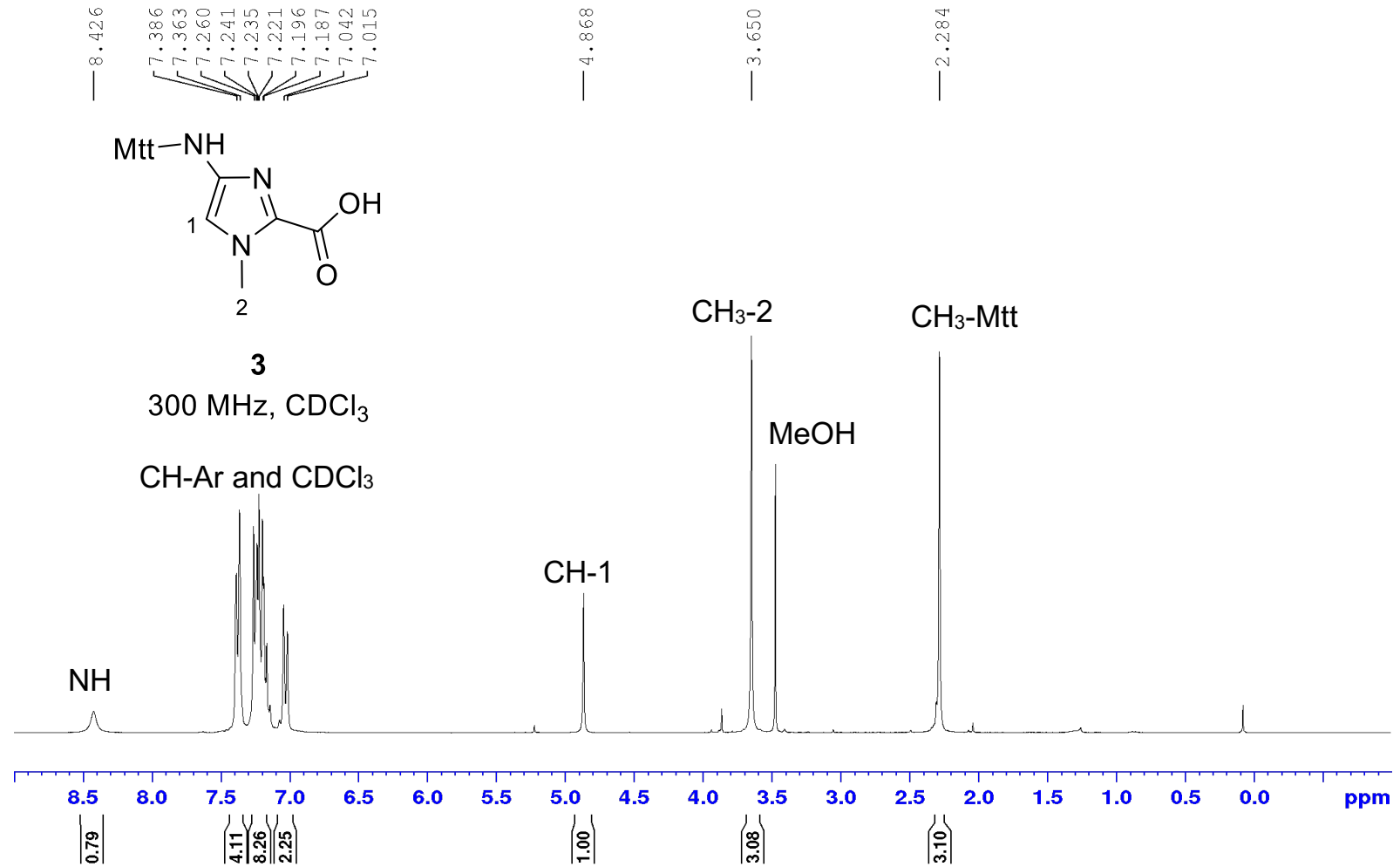

Figure S37: ${ }^{1} \mathrm{H}-\mathrm{NMR}(300 \mathrm{MHz})$ spectrum of Mtt-Im-OH (3).

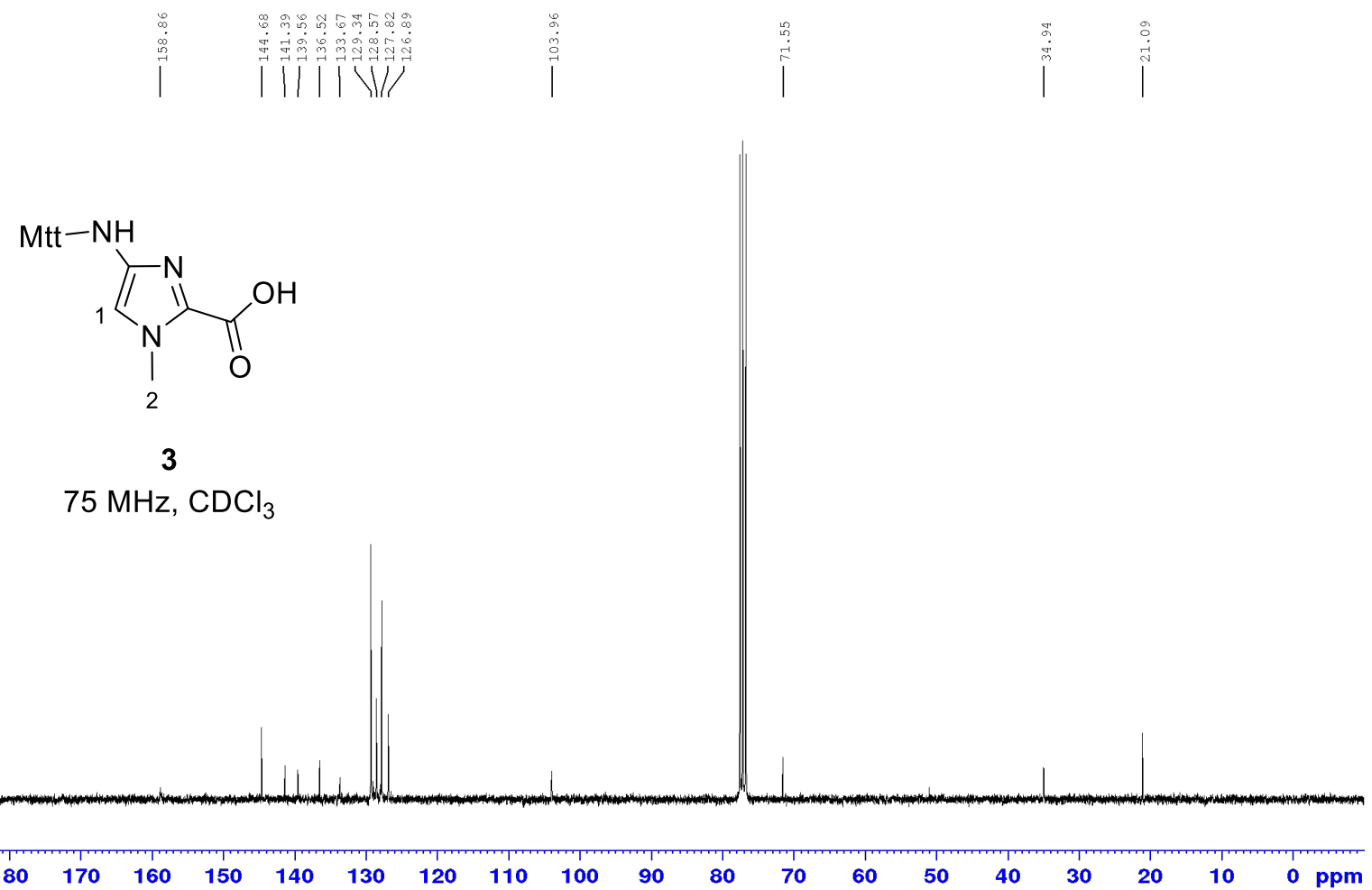

Figure S38: ${ }^{13} \mathrm{C}-\mathrm{NMR}(75 \mathrm{MHz})$ spectrum of Mtt-Im-OH (3). 


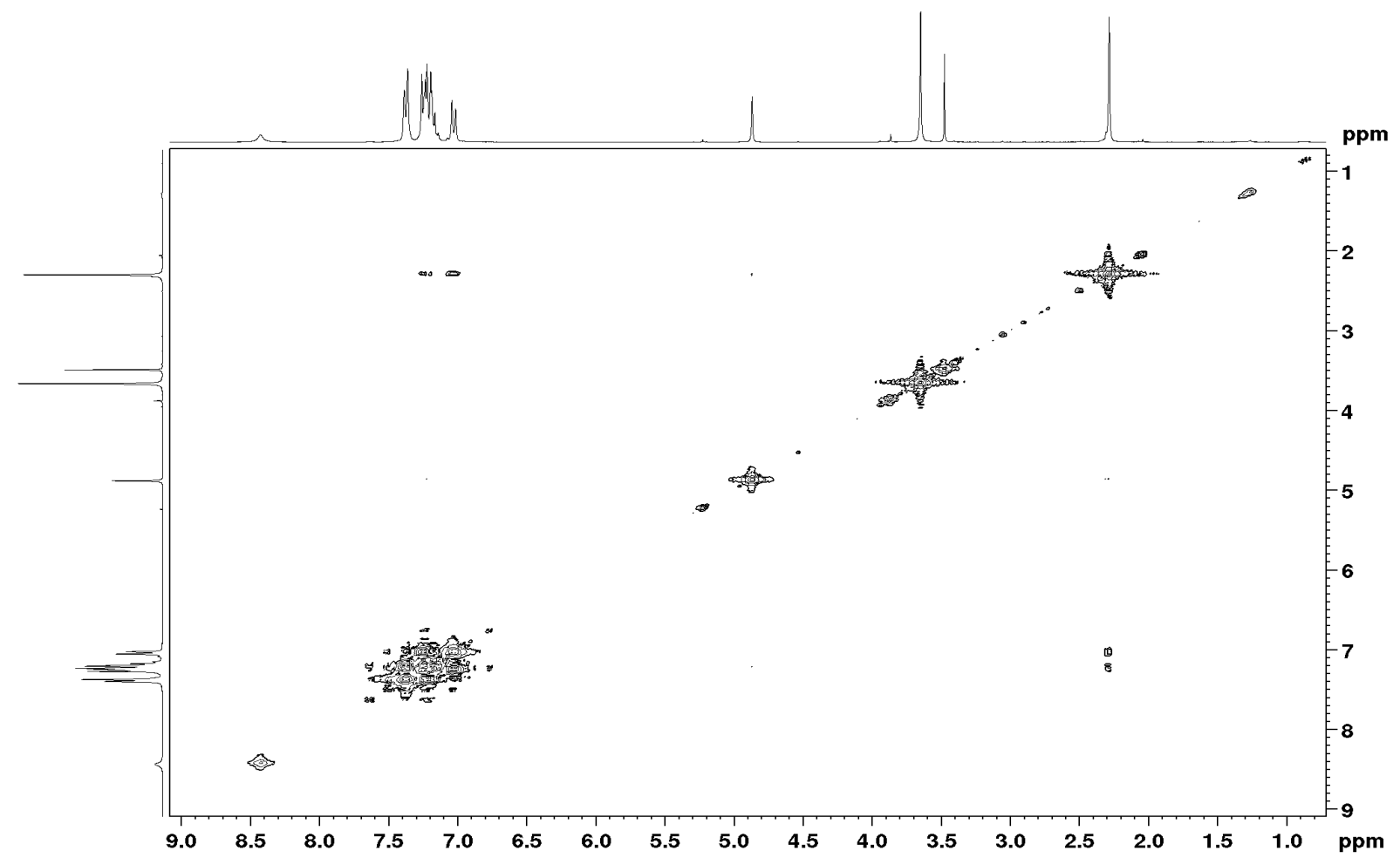

Figure S39: COSY-NMR (300 MHz) spectrum of Mtt-Im-OH (3).

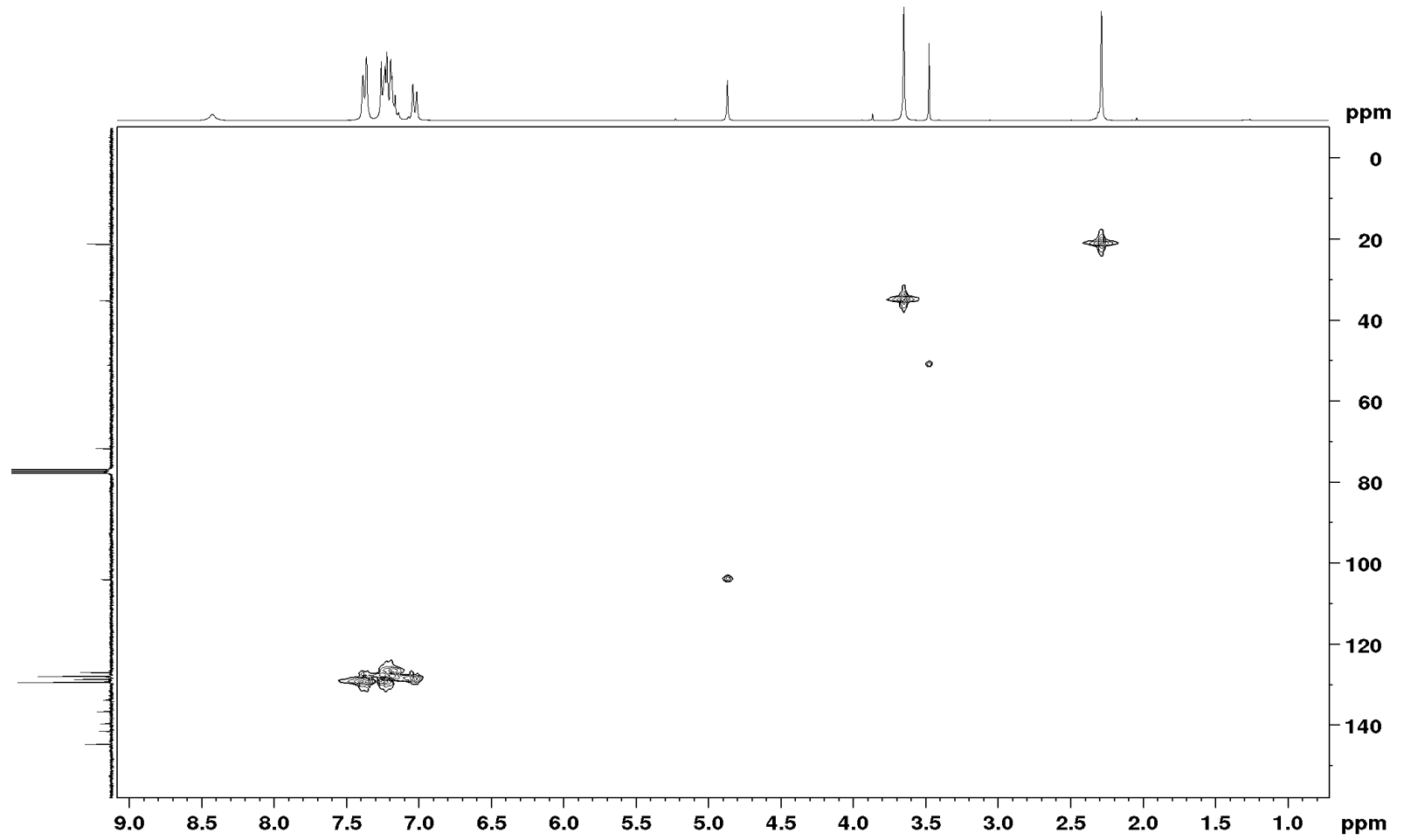

Figure S40: HSQC-NMR (300 MHz, 75 MHz) spectrum of Mtt-Im-OH (3). 


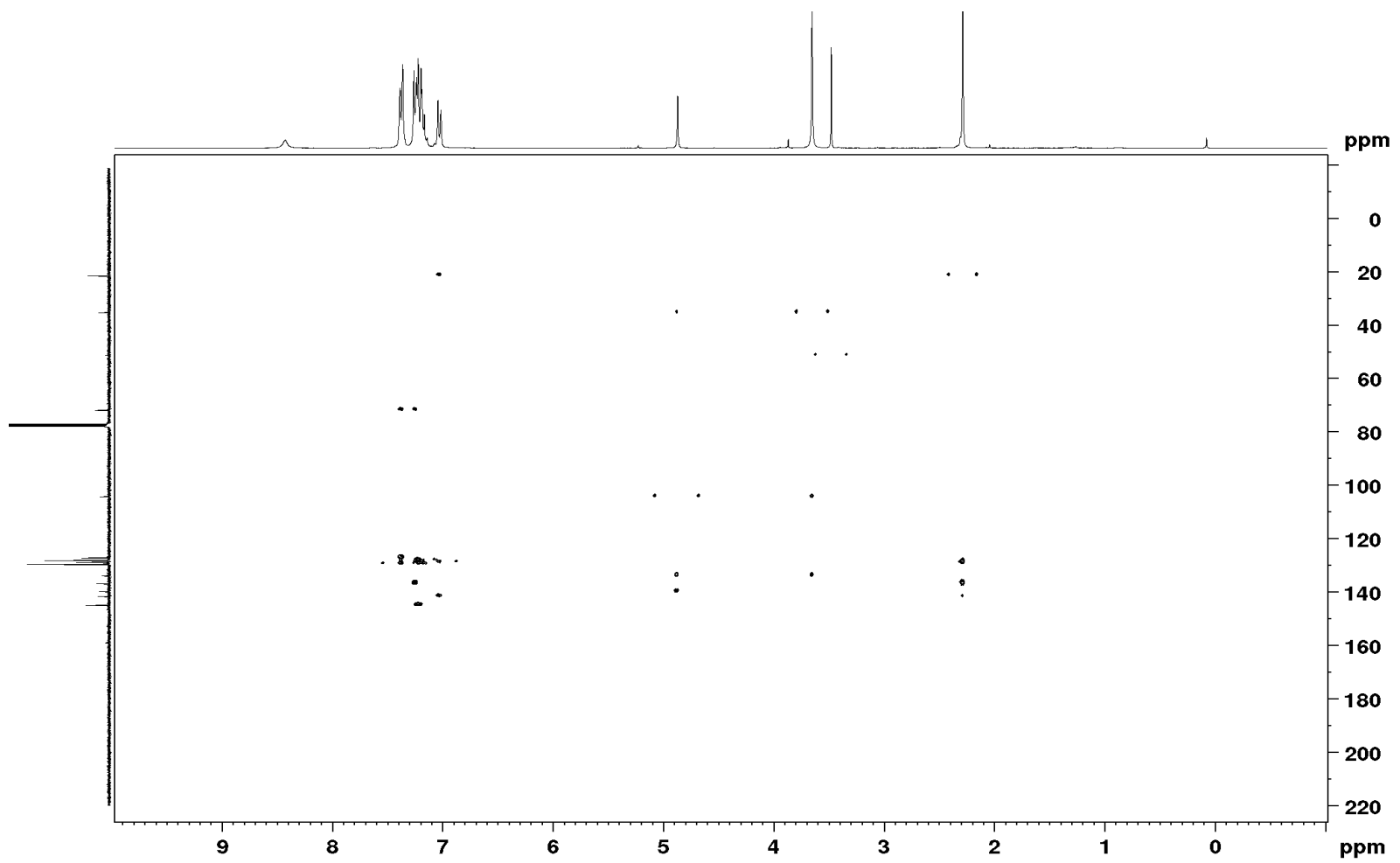

Figure S41: HMBC-NMR (500 MHz, $125 \mathrm{MHz}$ ) spectrum of Mtt-Im-OH (3).

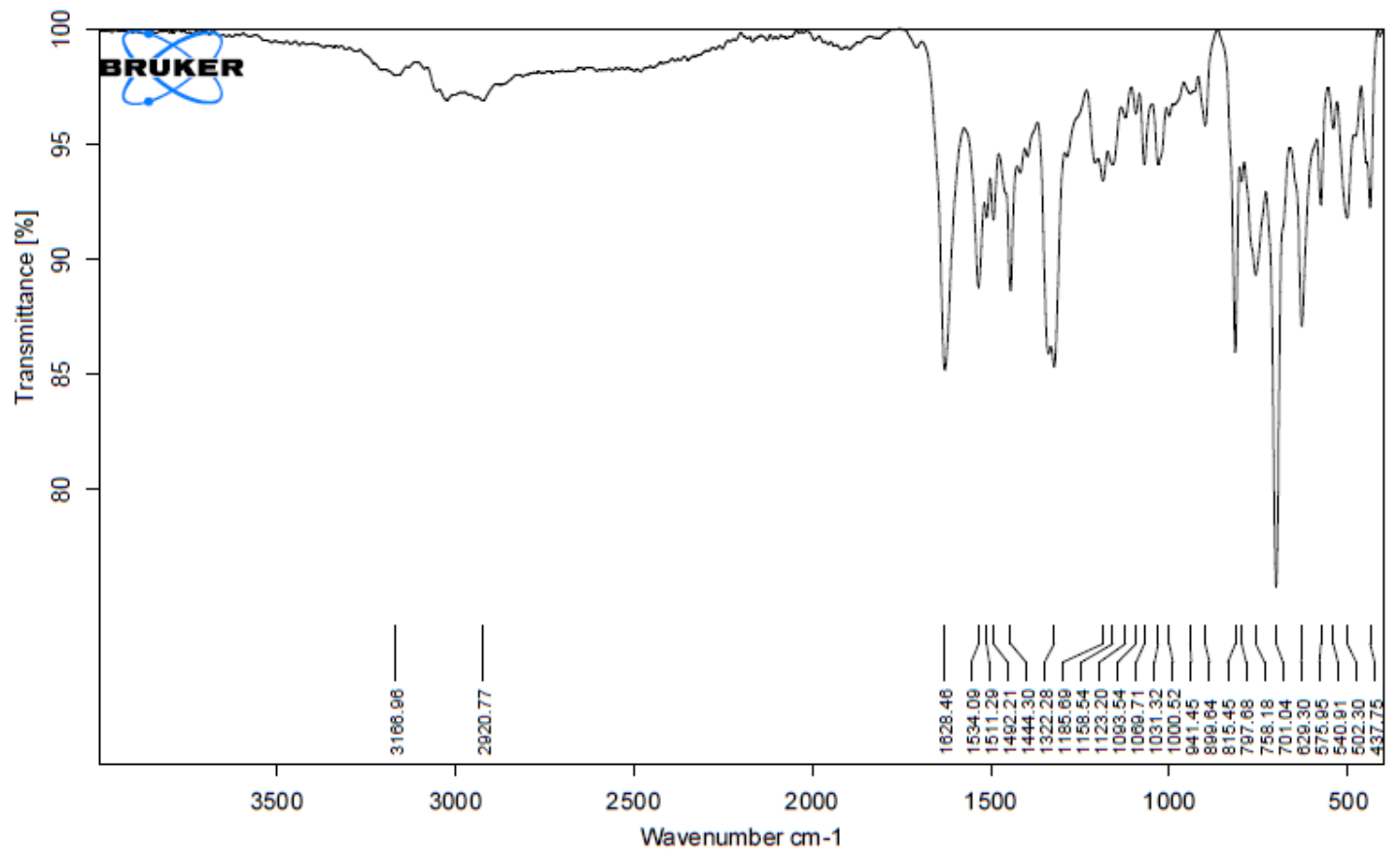

Figure S42: IR spectrum of Mtt-Im-OH (3). 

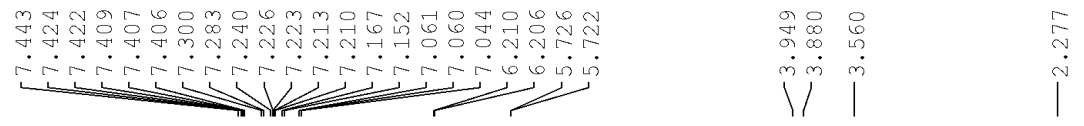<smiles>[M]CNc1ccc(C(=O)Nc2cn(S)c(C(=O)O[2H])n2)n1C</smiles>

33

$500 \mathrm{MHz}, \mathrm{CD}_{3} \mathrm{OD}$

$\mathrm{CH}_{3}-6$

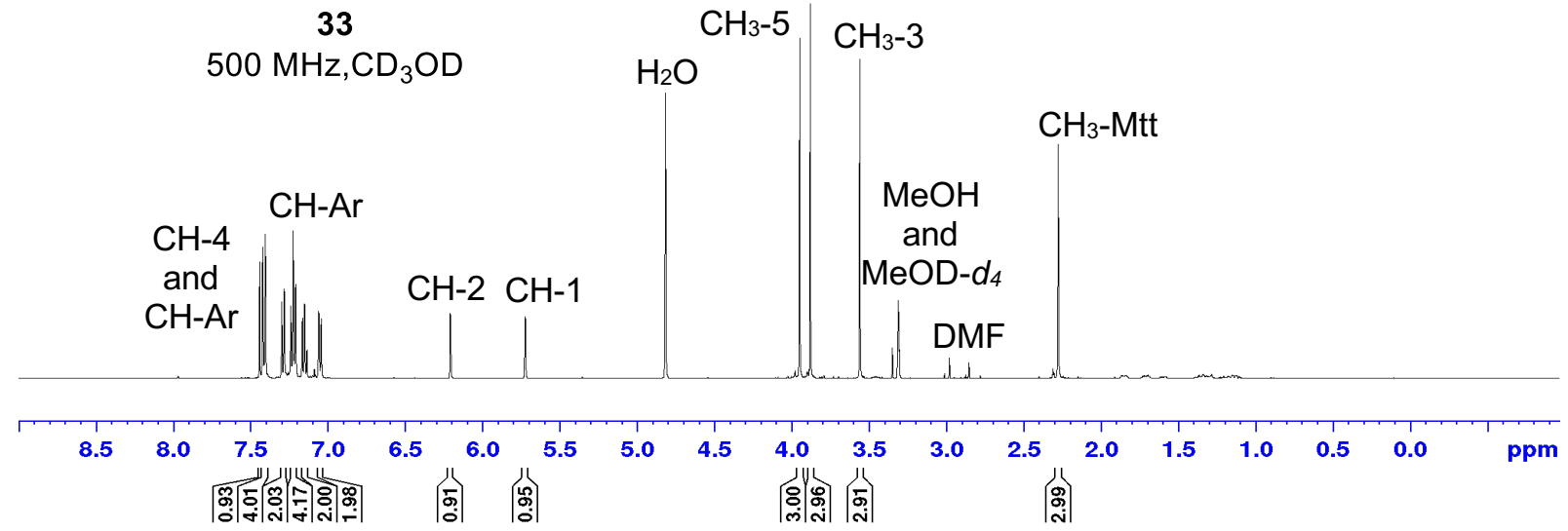

Figure S43: ${ }^{1} \mathrm{H}-\mathrm{NMR}(500 \mathrm{MHz})$ spectrum of Mtt-PyIm-OMe (33).

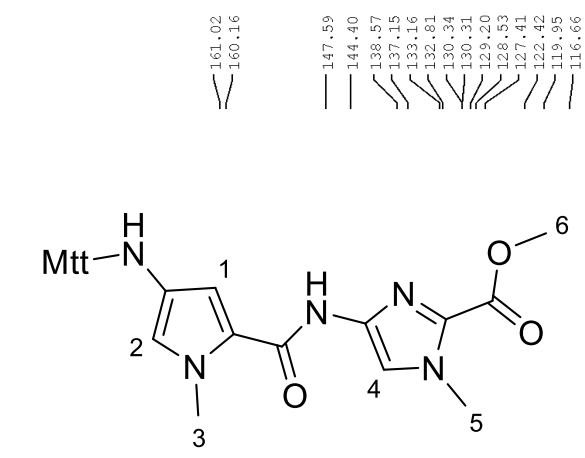

33

$125 \mathrm{MHz}, \mathrm{CD}_{3} \mathrm{OD}$

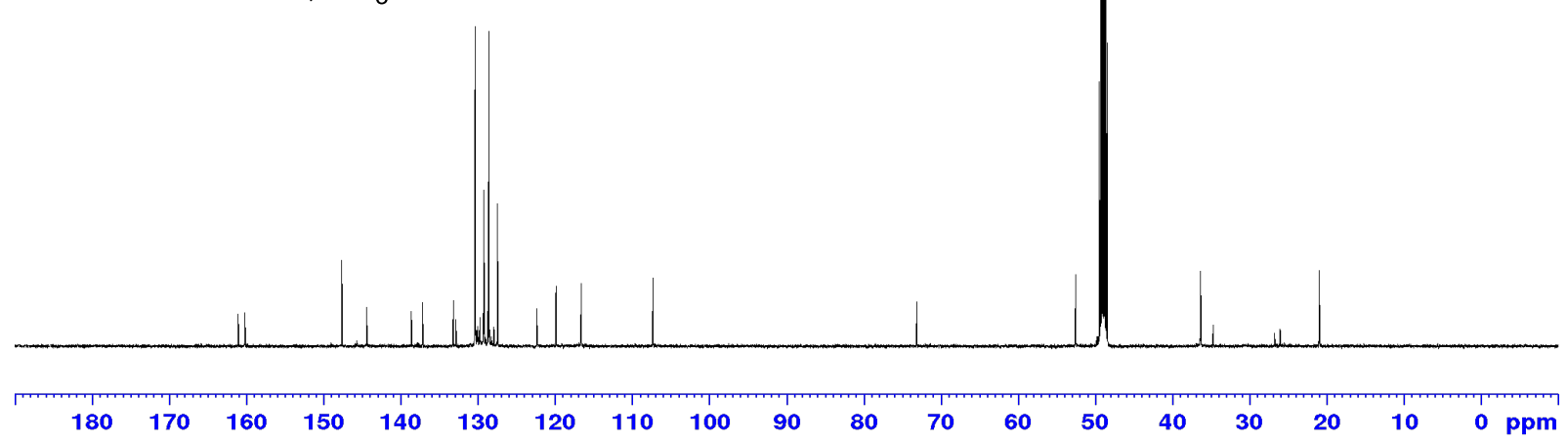

Figure S44: ${ }^{13} \mathrm{C}-\mathrm{NMR}(125 \mathrm{MHz})$ spectrum of Mtt-PyIm-OMe (33). 


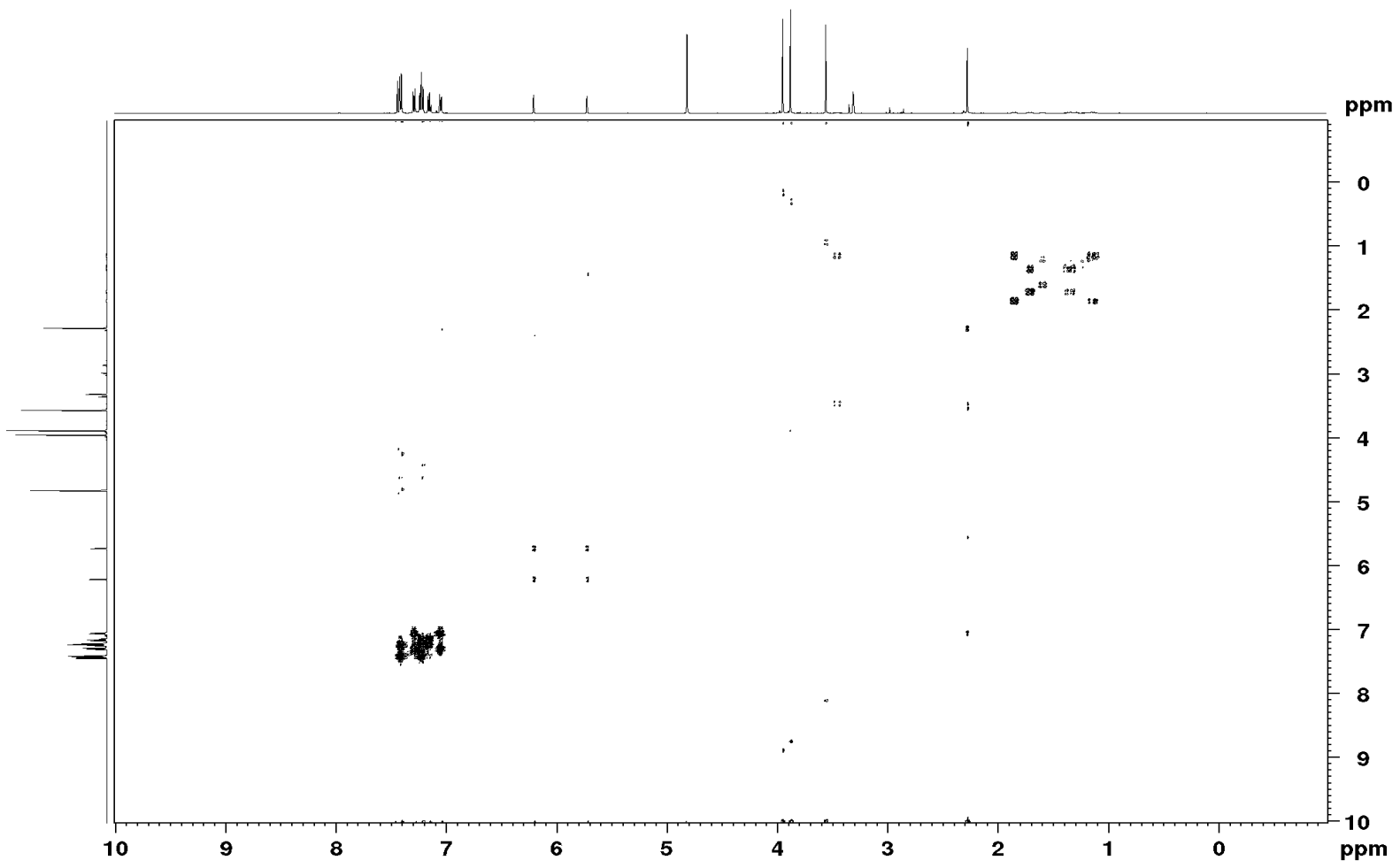

Figure S45: COSY-NMR (500 MHz) spectrum of Mtt-PyIm-OMe (33).

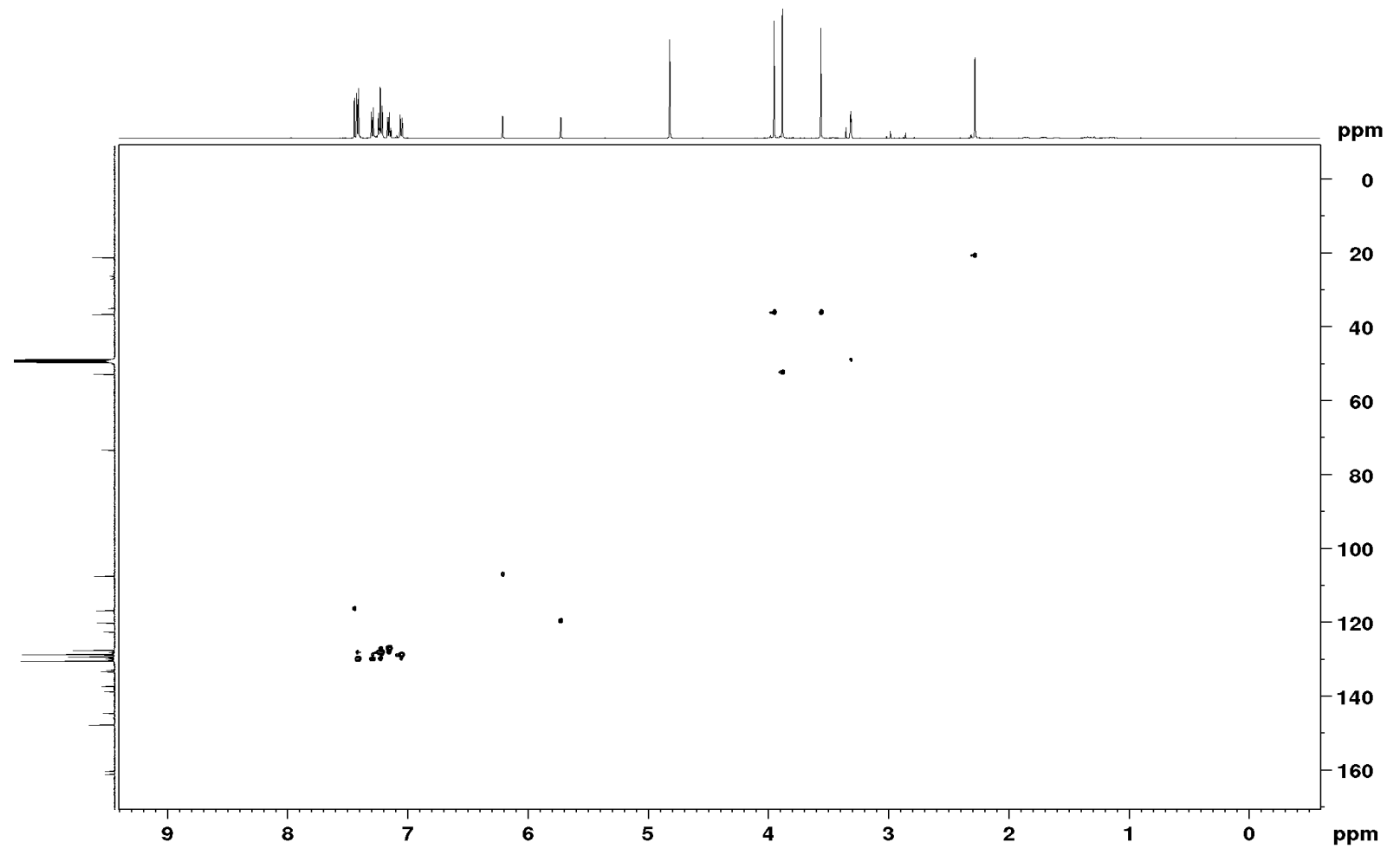

Figure S46: HSQC-NMR (500 MHz, 125 MHz) spectrum of Mtt-PyIm-OMe (33). 


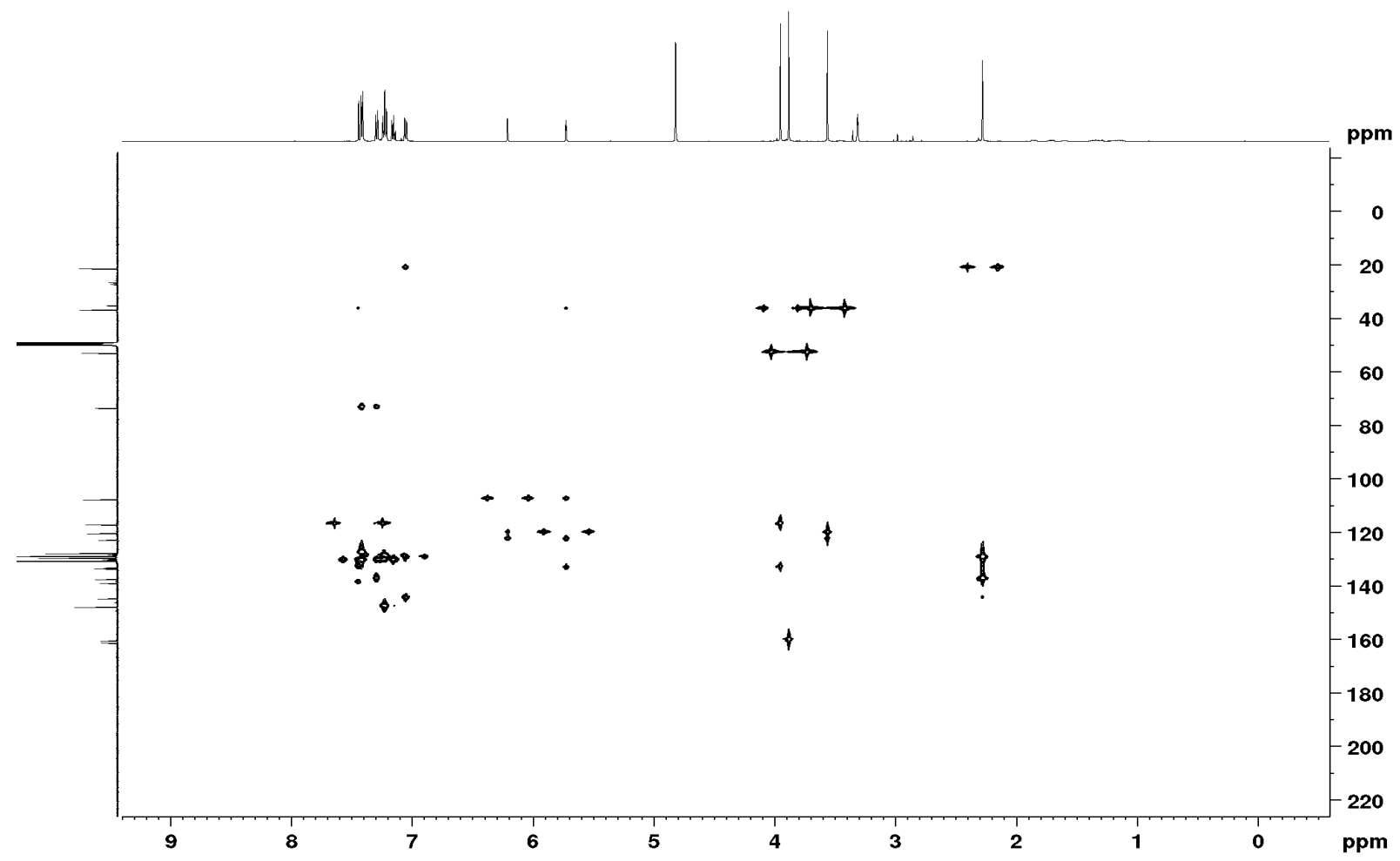

Figure S47: HMBC-NMR (500 MHz, 125 MHz) spectrum of Mtt-PyIm-OMe (33).

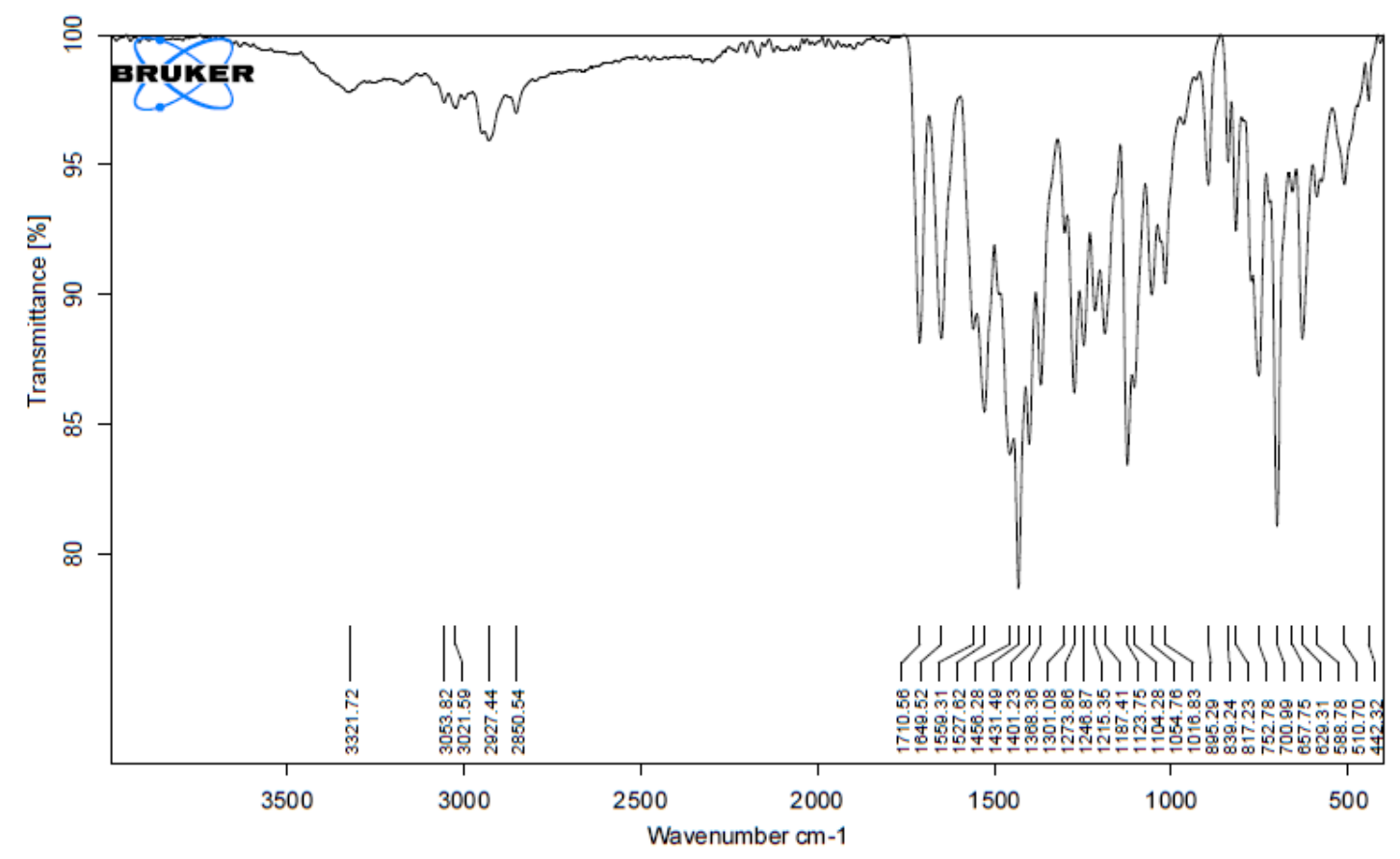

Figure S48: IR spectrum of Mtt-PyIm-OMe (33). 

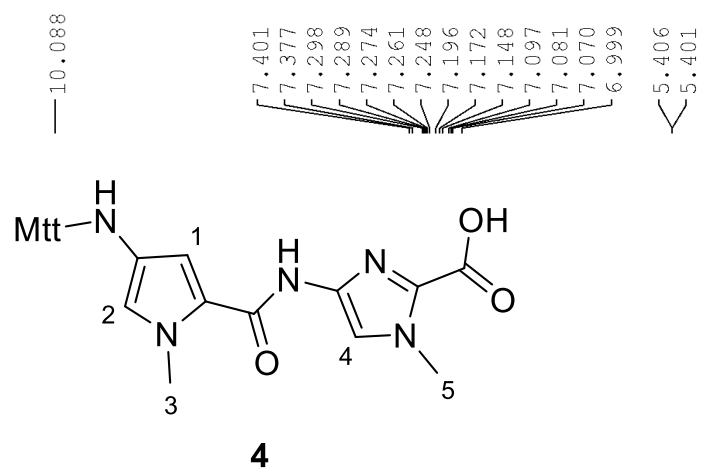

$300 \mathrm{MHz}, \mathrm{DMSO}-\mathrm{d} 6$

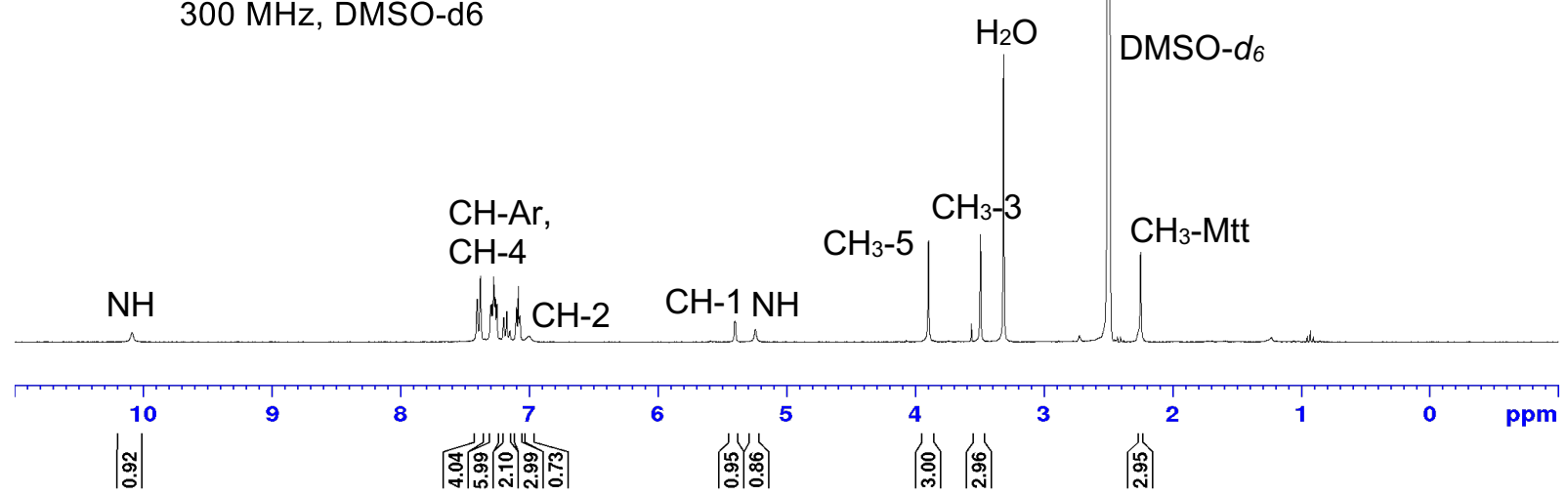

Figure S49: ${ }^{1} \mathrm{H}-\mathrm{NMR}(300 \mathrm{MHz})$ spectrum of Mtt-PyIm-OH (4).

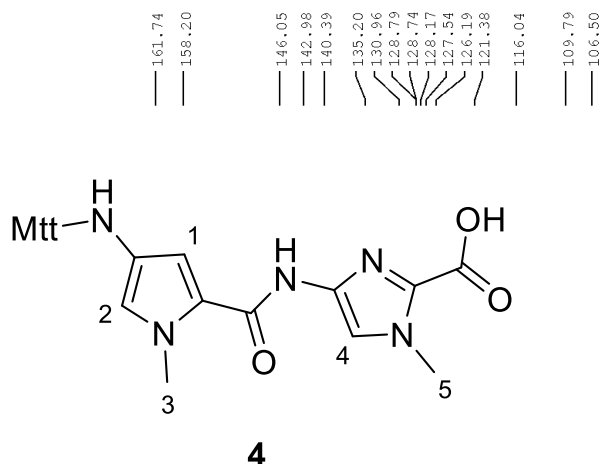

$125 \mathrm{MHz}$, DMSO-d6

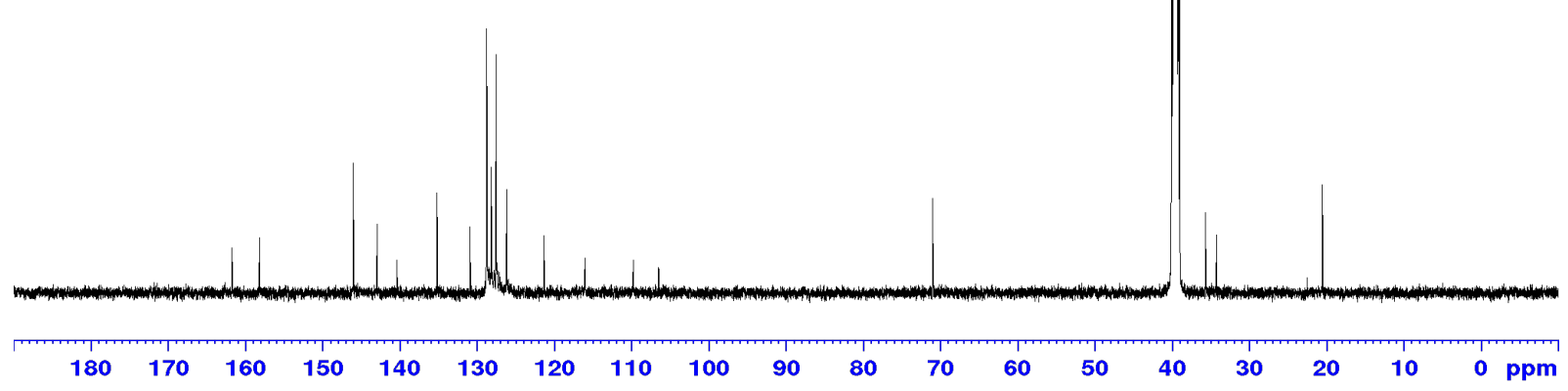

Figure S50: ${ }^{13} \mathrm{C}-\mathrm{NMR}(125 \mathrm{MHz})$ spectrum of Mtt-PyIm-OH (4). 


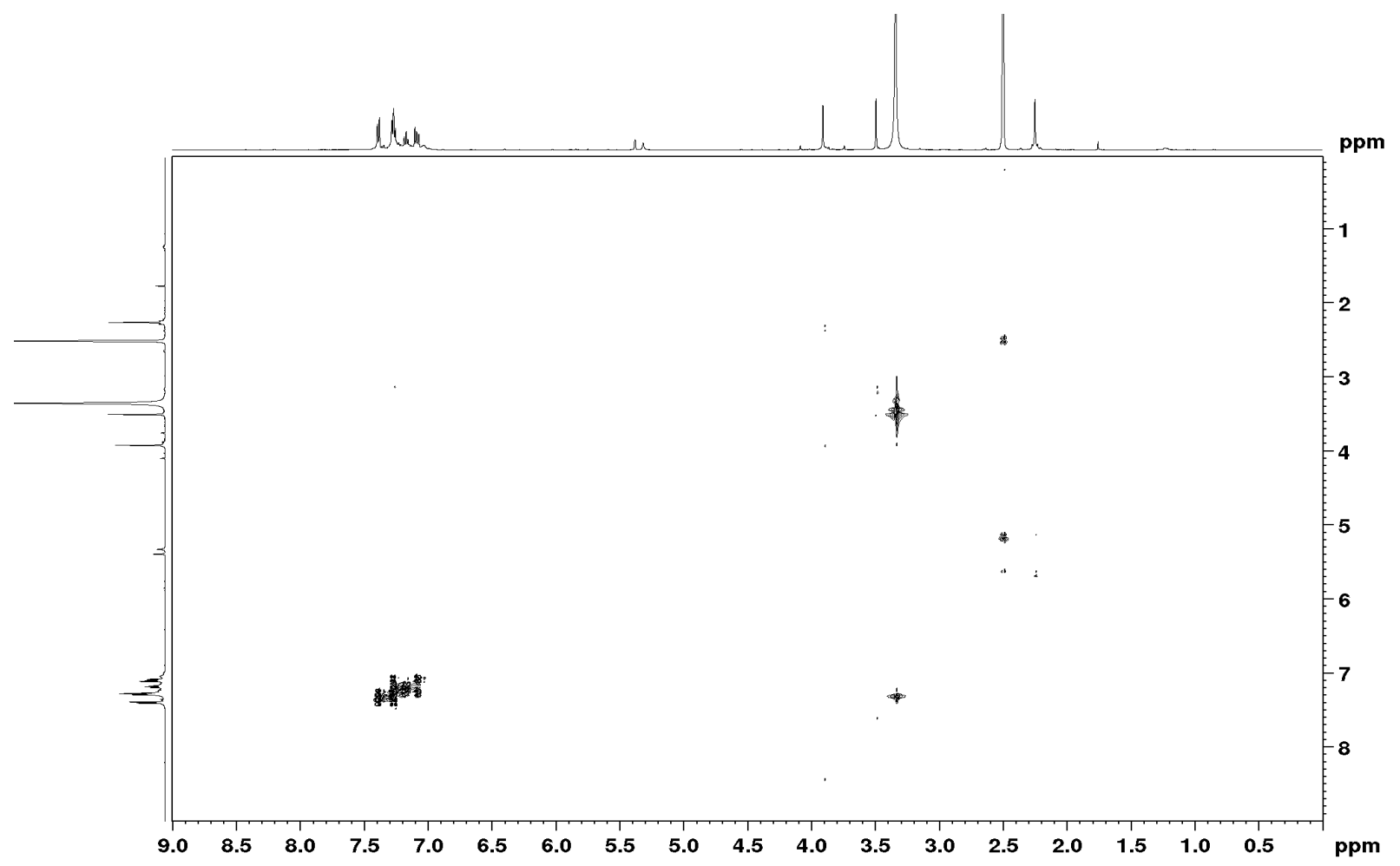

Figure S51: COSY-NMR (500 MHz) spectrum of Mtt-PyIm-OH (4).

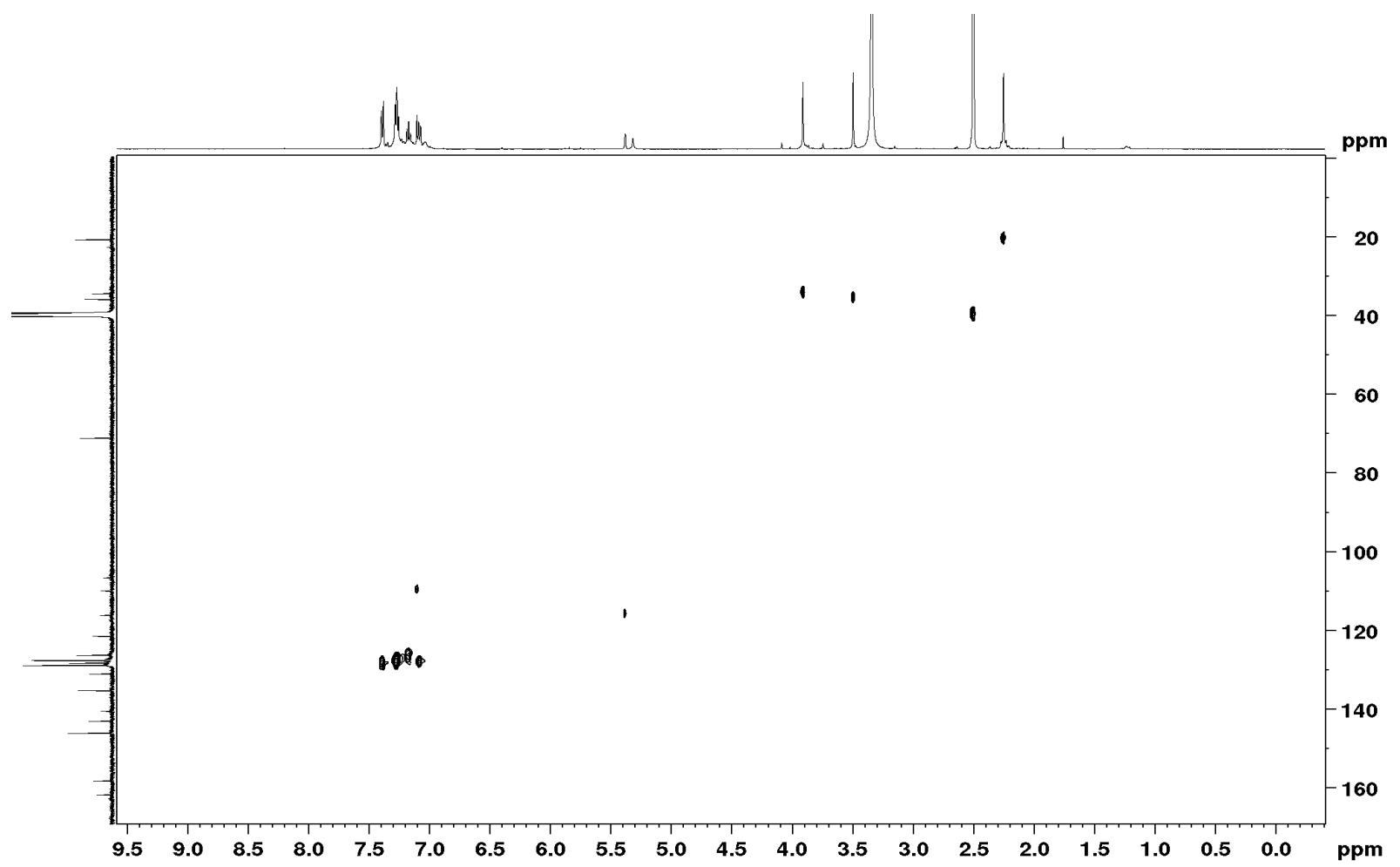

Figure S52: HSQC-NMR (500 MHz, $125 \mathrm{MHz}$ ) spectrum of Mtt-PyIm-OH (4). 


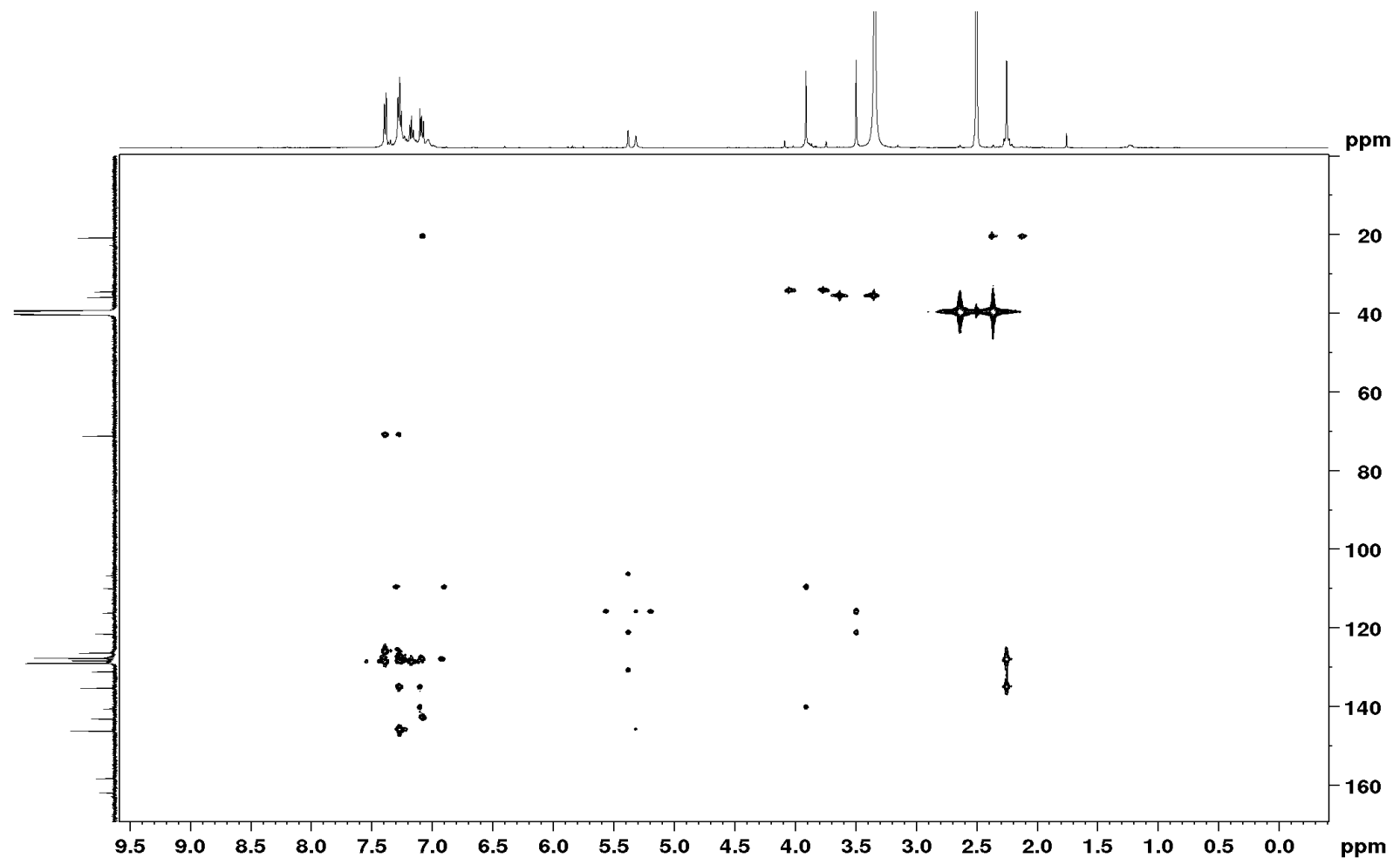

Figure S53: HMBC-NMR (500 MHz, 125 MHz) spectrum of Mtt-PyIm-OH (4).

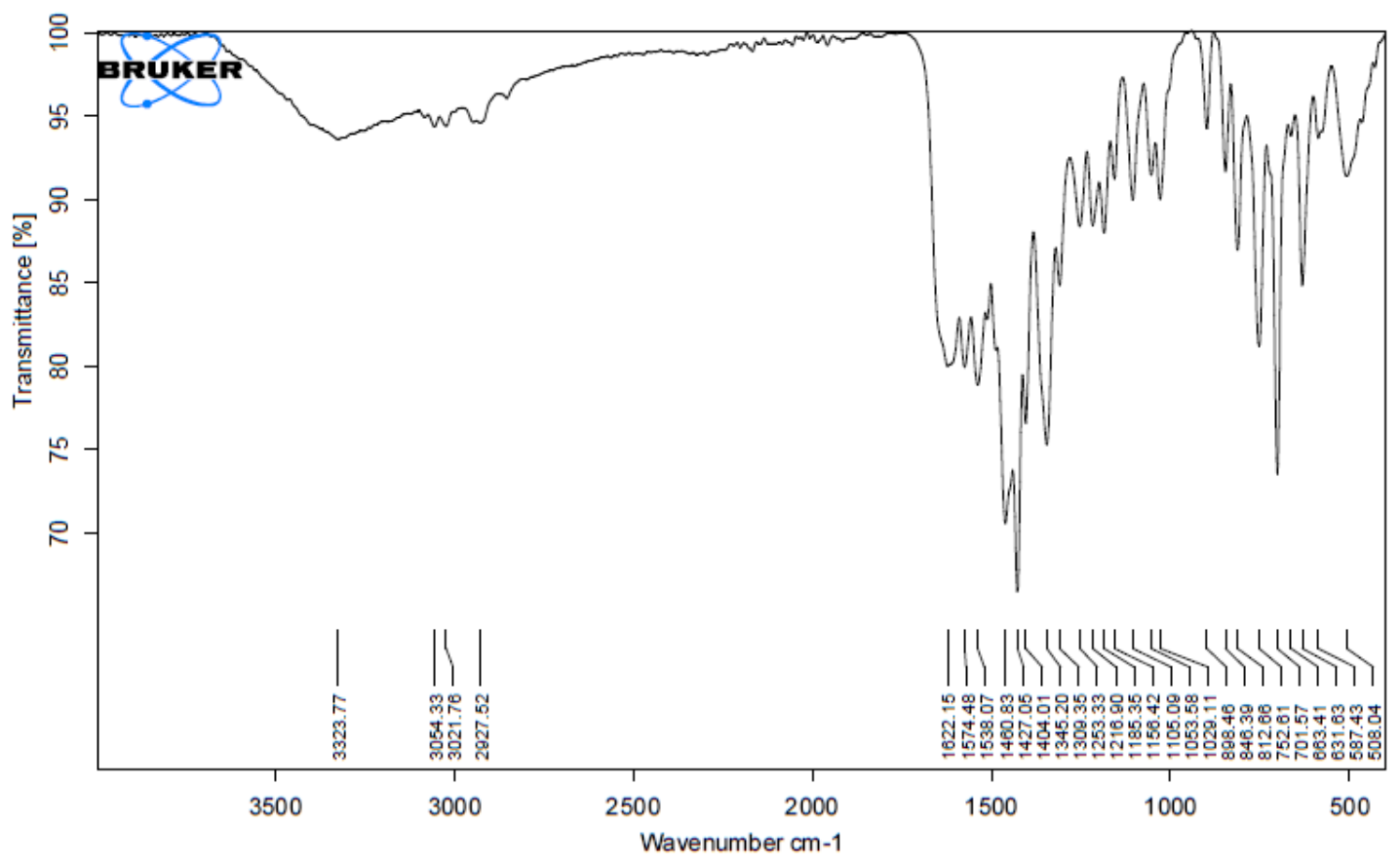

Figure S54: IR spectrum of Mtt-PyIm-OH (4). 


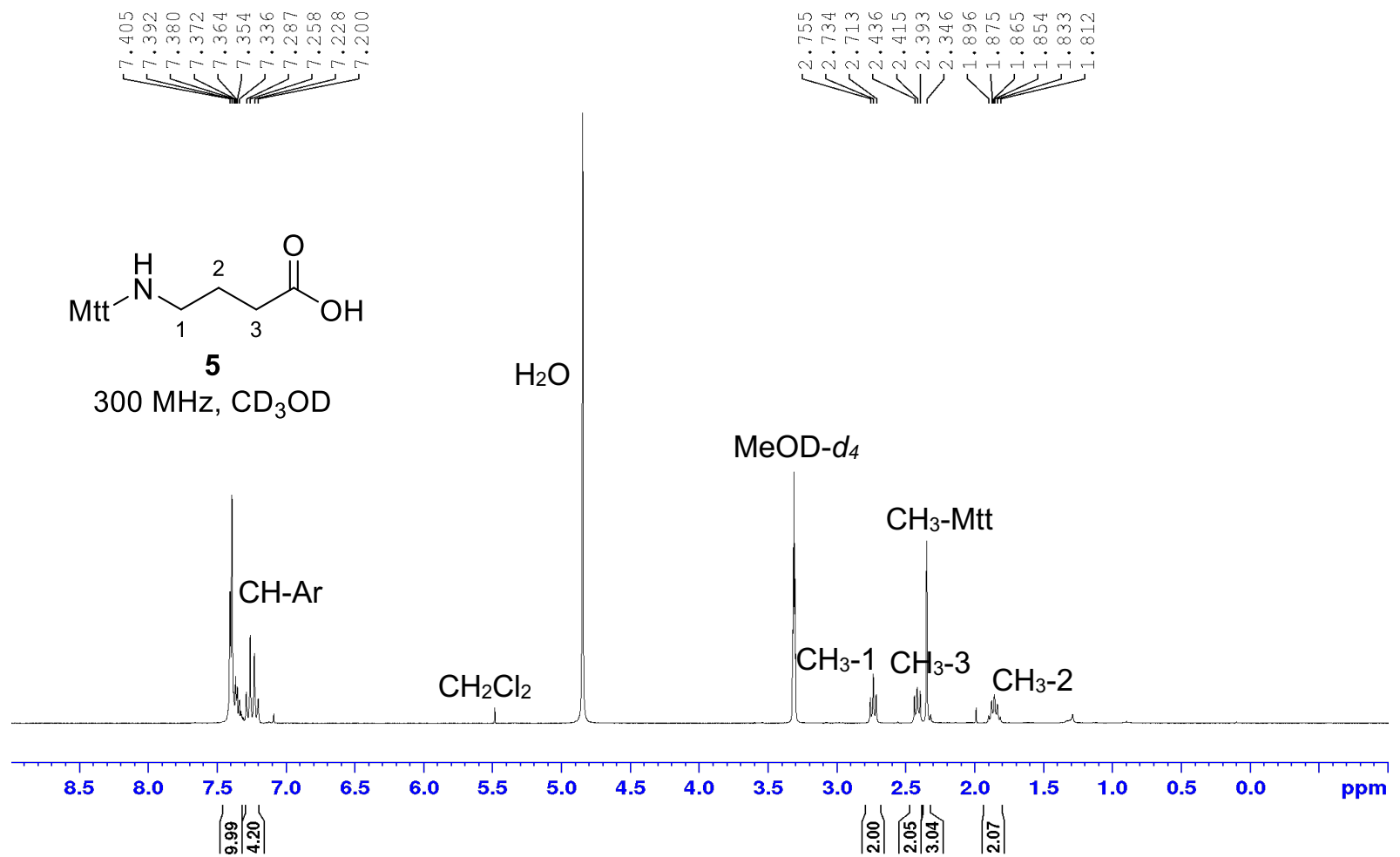

Figure S55: ${ }^{1} \mathrm{H}-\mathrm{NMR}(300 \mathrm{MHz})$ spectra of Mtt- $\gamma-\mathrm{OH}(\mathbf{5})$.
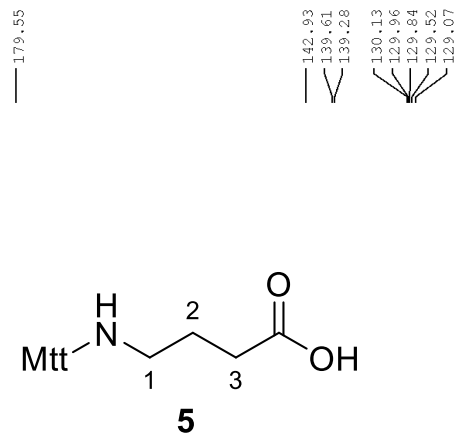

$75 \mathrm{MHz}, \mathrm{CD}_{3} \mathrm{OD}$

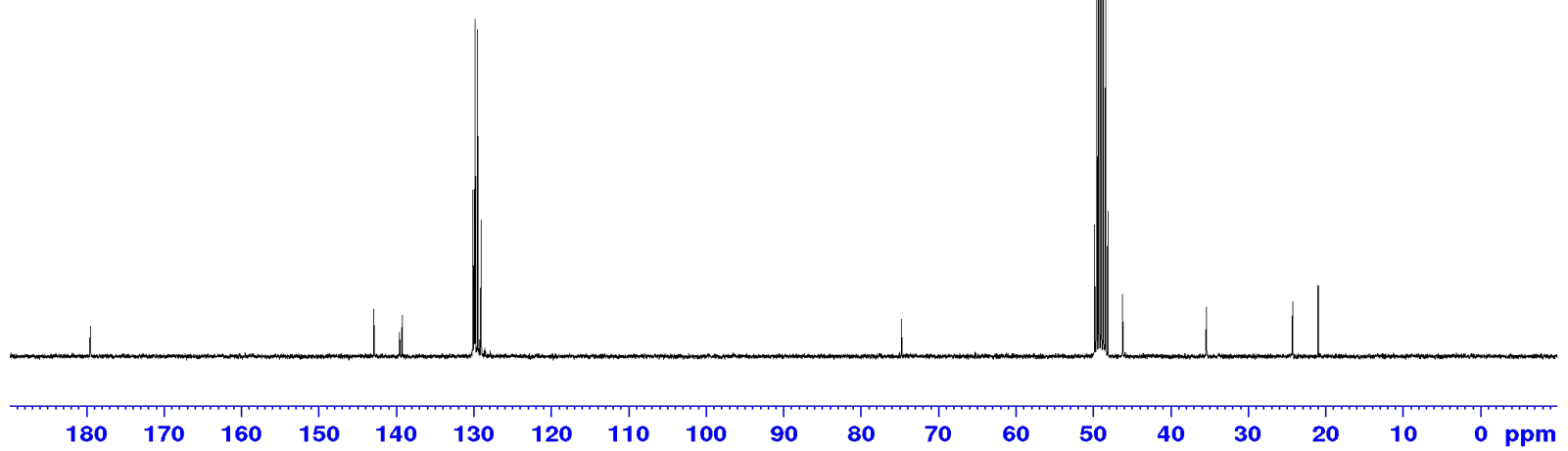

Figure S56: ${ }^{13} \mathrm{C}-\mathrm{NMR}(75 \mathrm{MHz})$ spectrum of $\mathrm{Mtt}-\gamma-\mathrm{OH}(\mathbf{5})$. 


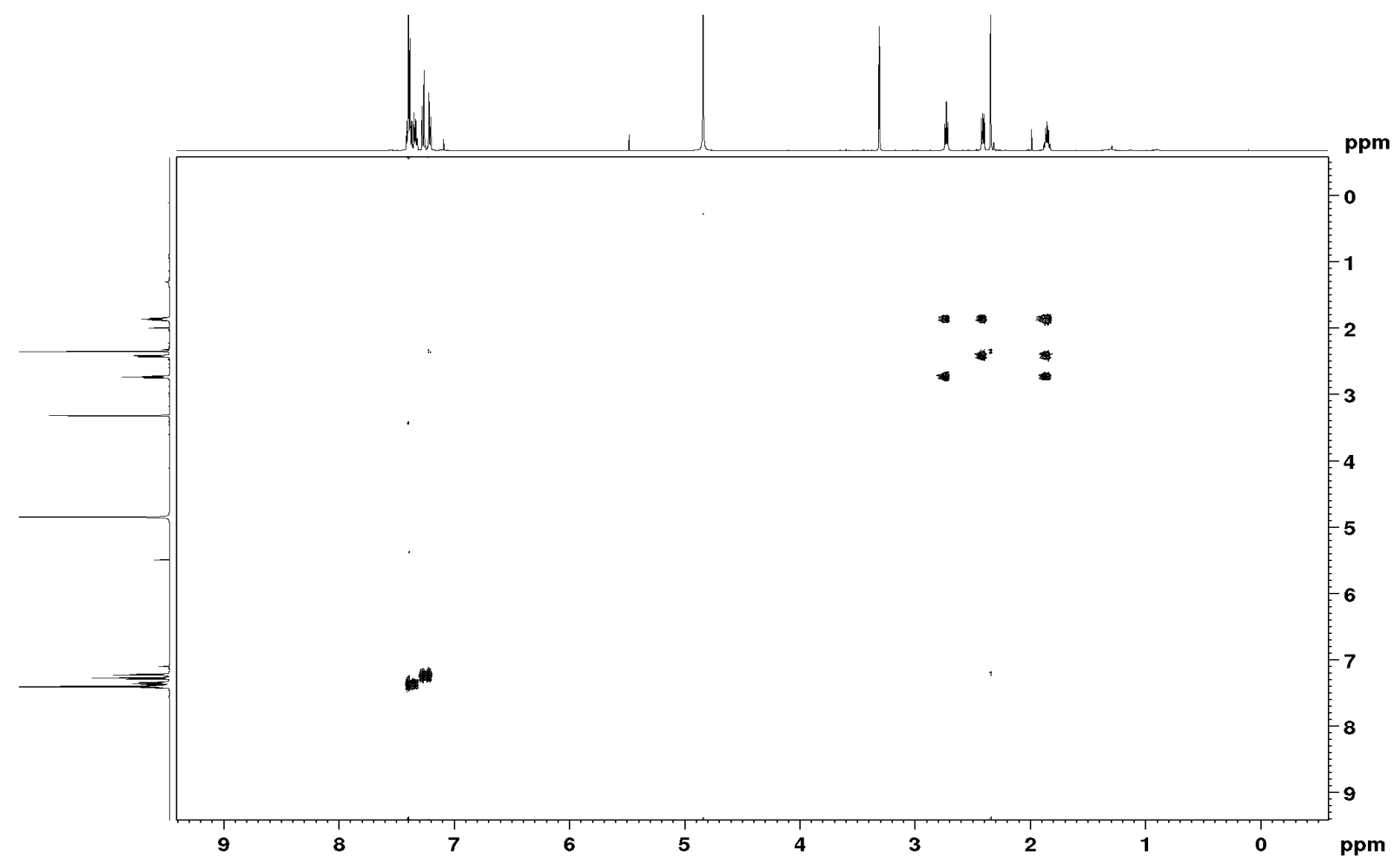

Figure S57: COSY-NMR (500 MHz) spectrum of Mtt- $\gamma-\mathrm{OH}$ (5).

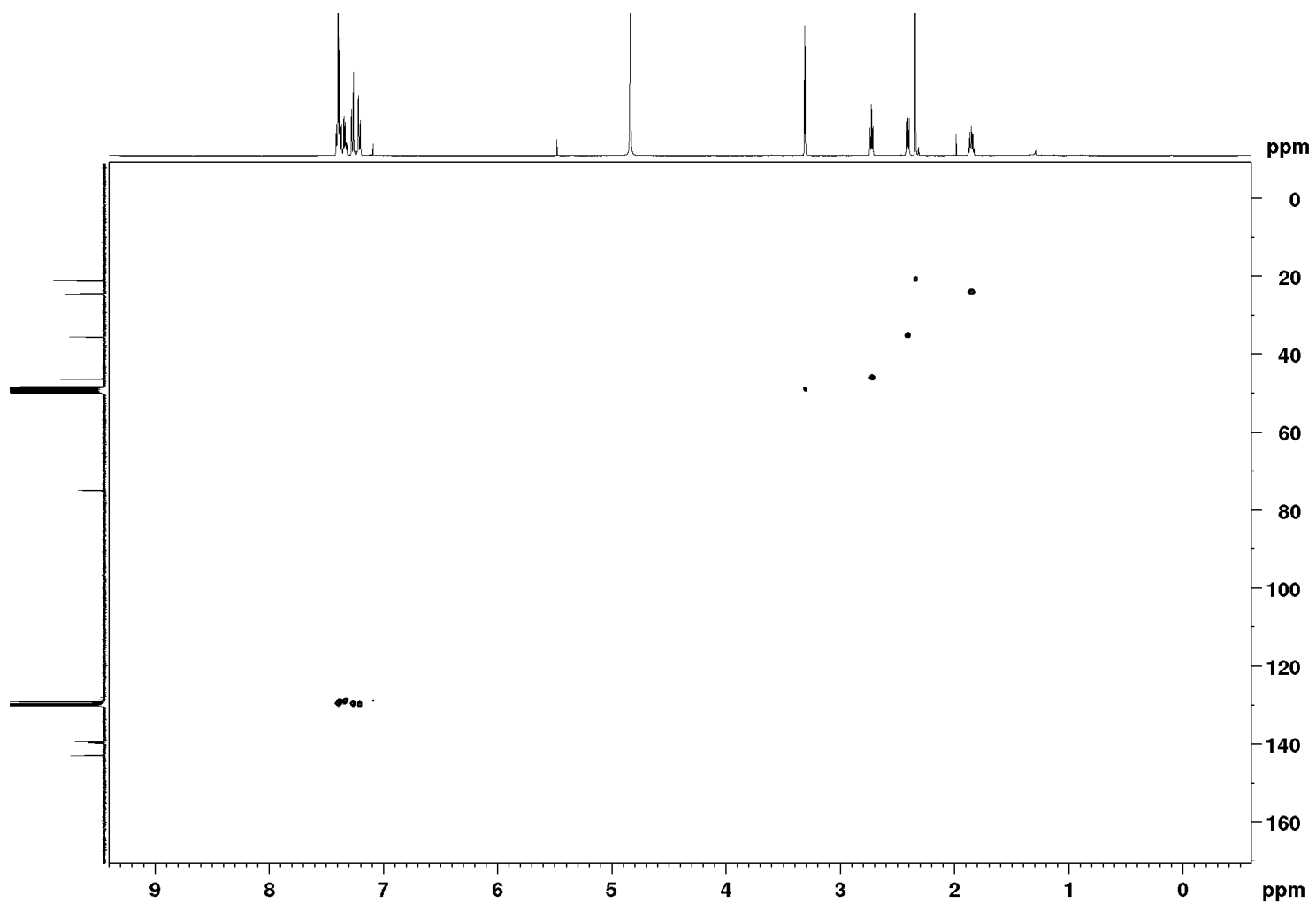

Figure S58: HSQC-NMR (500 MHz, $75 \mathrm{MHz}$ ) spectrum of Mtt- $\gamma-\mathrm{OH}$ (5). 


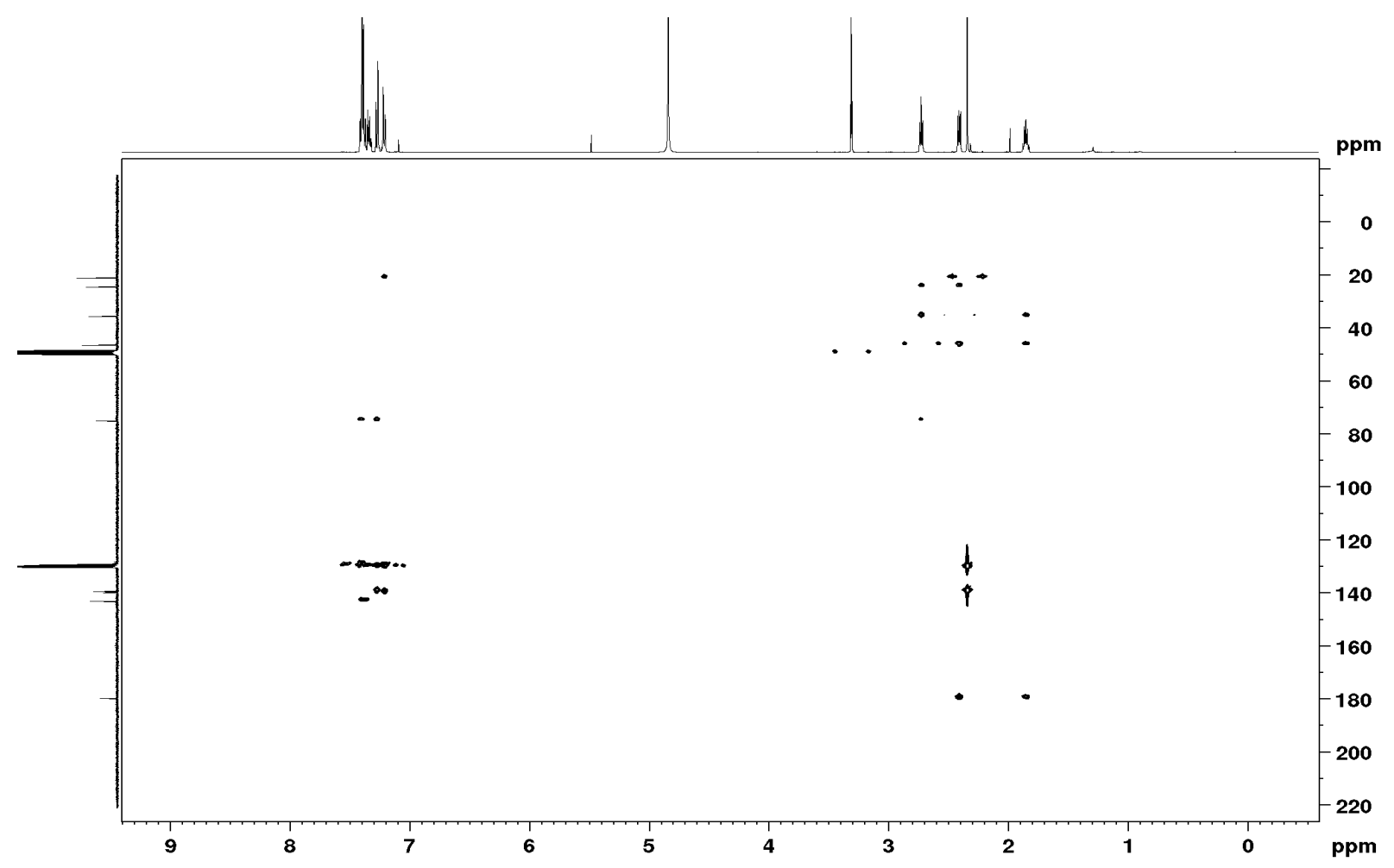

Figure S59: HMBC-NMR (500 MHz, $75 \mathrm{MHz}$ ) spectrum of Mtt- $\gamma-\mathrm{OH}$ (5).

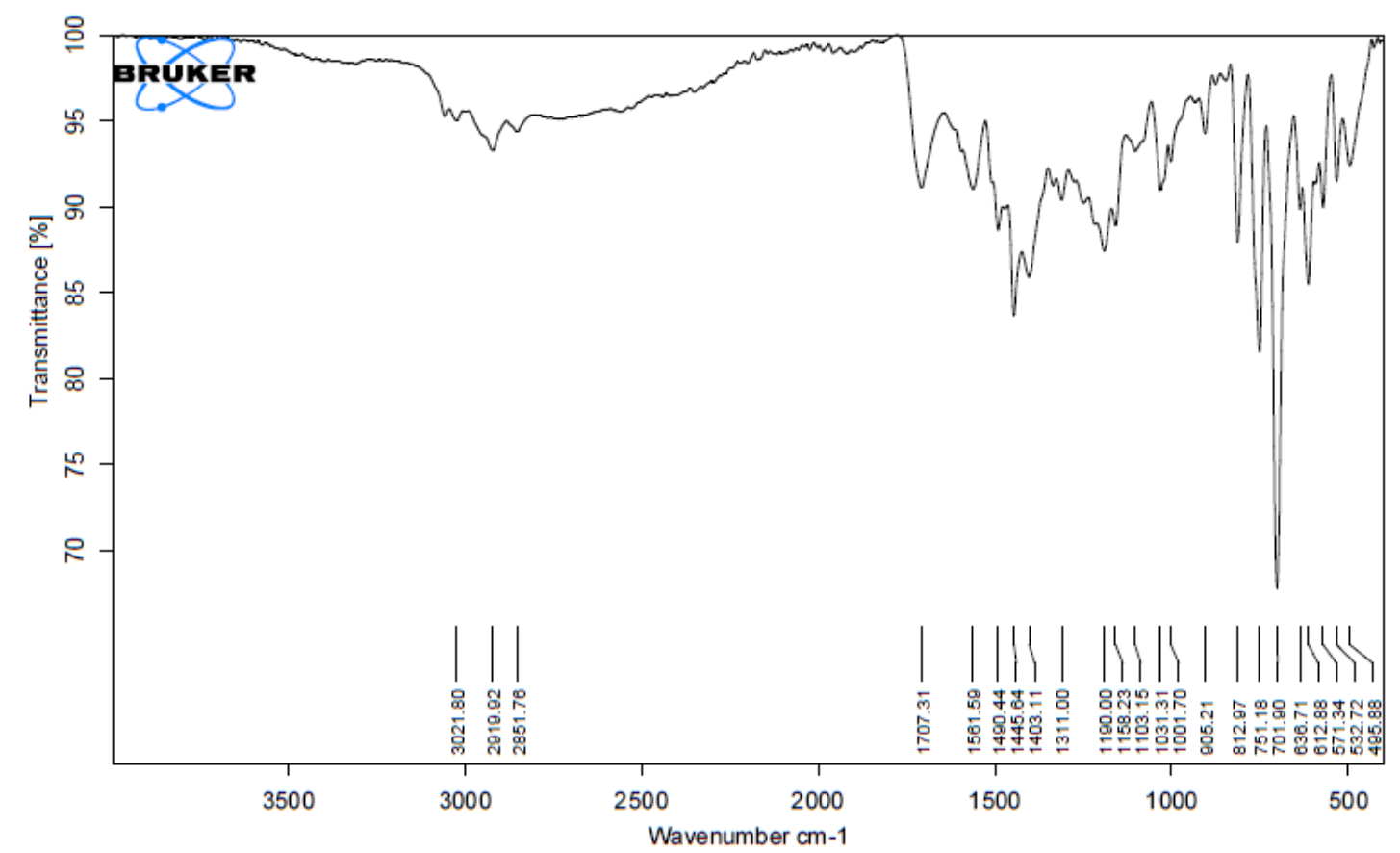

Figure S60: IR spectrum of Mtt- $\gamma-\mathrm{OH}(5)$. 


\section{Solid Phase Peptide Synthesis}

Both, Fmoc-SPPS and Mtt-SPPS, follow the same protocol, except the deprotection step. Further modifications are described below.

The polyamide hairpins were synthesized manually in $2 \mathrm{~mL}$ polypropylene reactors with plunger and frit, (pore size $25 \mu \mathrm{m}$, MultiSynTech GmbH; Germany). TG R PHB resin was preloaded with Fmoc- $\beta$ Ala-OH and the loading determined before further couplings.

Loading of the first amino acid: ${ }^{[\mathrm{xix}]}$ Under inert atmosphere, TG R PHB resin (500 mg, $0.18-0.23 \mathrm{mmol} / \mathrm{g}$ ) was placed in a flask, covered with DMF $(3.0 \mathrm{~mL})$ and swelled for $30 \mathrm{~min}$. In a second flask, under inert atmosphere, Fmoc- $\beta$ Ala-OH (358 mg, $1.15 \mathrm{mmol})$ was dissolved in $\mathrm{CH}_{2} \mathrm{Cl}_{2}(5.0 \mathrm{~mL})$ and $\mathrm{DMF}(100 \mu \mathrm{L})$ and the solution cooled to $0{ }^{\circ} \mathrm{C}$ before adding DIC $(89.0 \mu \mathrm{L}, 575 \mu \mathrm{mol})$. After $20 \mathrm{~min}$, the solvent was removed, the residue dissolved in DMF $(3.0 \mathrm{~mL}$ ) and added to the swelled resin. DMAP (14.0 mg, $115 \mu \mathrm{mol})$ dissolved in DMF $(50 \mu \mathrm{L})$ was added and the mixture was shaken under inert atmosphere for $1 \mathrm{~h}$. The resin was filtered off and washed with DMF $(5 \times 0.5 \mathrm{~mL}), \mathrm{CH}_{2} \mathrm{Cl}_{2}(10 \times 0.5 \mathrm{~mL})$ and DMF $(5 \times$ $0.5 \mathrm{~mL})$. Lutidine $/ \mathrm{Ac}_{2} \mathrm{O} / \mathrm{DMF}$ 6:5:89 $(2.0 \mathrm{~mL})$ was added for $5 \mathrm{~min}$. The resin was filtered off and washed with DMF $(5 \times 0.5 \mathrm{~mL})$ and $\mathrm{CH}_{2} \mathrm{Cl}_{2}(10 \times 0.5 \mathrm{~mL})$. It was dried under reduced pressure and the loading determined.

Determination of the resin loading: The Fmoc-group of a weighted amount of resin $(\sim 5.00 \mathrm{mg})$, previously loaded with the first amino acid, Fmoc- $\beta$ Ala-OH, was deprotected by adding twice a solution of $20 \%$ piperidine in DMF $(500 \mu \mathrm{L})$ for $5 \mathrm{~min}$. The filtrates were collected and the absorbance measured at $300 \mathrm{~nm}$ $\left(\varepsilon_{300 \mathrm{~nm}}(\text { Fmoc })=7800 \mathrm{M}^{-1} \mathrm{~cm}^{-1}\right)^{[\mathrm{xx}]}$ on a Tecan $20 \mathrm{M}$ Spark plate reader and the loading was calculated.

\section{Manual Solid Phase Protocol for Peptide Synthesis}

The amounts of reagents of the following synthesis protocol correspond to $10 \mu \mathrm{mol}$ scale. For higher scales, the reagents were scaled up correspondingly. For shaking an Edmund Bühler Swip shaker was used.

Swelling: $10 \mu \mathrm{mol}$ of resin was swollen in $0.5 \mathrm{~mL} \mathrm{DMF}$ for $30 \mathrm{~min}$.

Deprotection of the temporal Fmoc group: Piperidine (20\% in DMF, $250 \mu \mathrm{L})$ was added to the resin and shaken for $5 \mathrm{~min}$. This step was repeated, the resin filtered off and washed with DMF ( $5 \mathrm{x} 0.5 \mathrm{~mL}), \mathrm{CH}_{2} \mathrm{Cl}_{2}$ $(5 \times 0.5 \mathrm{~mL})$ and DMF $(5 \times 0.5 \mathrm{~mL})$.

Deprotection of the temporal Mtt-group: TFA $\left(2 \%\right.$ in $\left.\mathrm{CH}_{2} \mathrm{Cl}_{2}, 250 \mu \mathrm{L}\right)$ was added to the resin and shaken for $20 \mathrm{~min}$. The resin was filtered off and washed with TFA ( $2 \%$ in $\left.\mathrm{CH}_{2} \mathrm{Cl}_{2}, 0.5 \mathrm{~mL}\right)$ until the washing solution was no longer coloured yellow. It was washed with $\mathrm{CH}_{2} \mathrm{Cl}_{2}(8 \times 0.5 \mathrm{~mL}), \mathrm{DMF}(8 \times 0.5 \mathrm{~mL})$ and the resin incubated with DIPEA $(10 \%$ in DMF, $250 \mu \mathrm{L})$ for 5 min to neutralize remaining acid.

Coupling of amino acids: The amino acid (4.00 eq) was dissolved in NMP (134 $\mu \mathrm{L})$ and DIPEA (8.00 eq for Fmoc-amino acids and 12.0 eq for Mtt-amino acids), and HATU (4.00 eq) was added. The resulting solution was activated for $3 \mathrm{~min}$ and added to the resin. This mixture was shaken for $1 \mathrm{~h}$ in the case of FmocPy-OH, Mtt-Py-OH, Fmoc- $\gamma-\mathrm{OH}$ and Mtt- $\gamma-\mathrm{OH}$ and $2 \mathrm{~h}$ in the case of Fmoc-Im-OH, Mtt-Im-OH, FmocPyIm-OH, Mtt-PyIm-OH and ImPy-OH. For the introduction of the amino acids after the $\gamma$-turn, DMSO/NMP 1:1 $(50 \mu \mathrm{L})$ was directly added to the coupling mixture after the corresponding coupling time for $30 \mathrm{~min}$. The resin was filtered off and washed with DMF $(5 \times 0.5 \mathrm{~mL}), \mathrm{CH}_{2} \mathrm{Cl}_{2}(5 \times 0.5 \mathrm{~mL})$ and DMF $(5 \times 0.5 \mathrm{~mL})$. 
Capping: After coupling of each amino acid, a solution of $\mathrm{Ac}_{2} \mathrm{O} /$ pyridine/DMF 5:5:90 $(250 \mu \mathrm{L})$ was added to the resin and it was shaken for $5 \mathrm{~min}$. The resin was filtered off and washed with DMF $(5 \times 0.5 \mathrm{~mL})$, $\mathrm{CH}_{2} \mathrm{Cl}_{2}(5 \times 0.5 \mathrm{~mL})$ and $\mathrm{DMF}(5 \times 0.5 \mathrm{~mL})$.

Final cleavage of the polyamide hairpins: The dried resin was treated with TFA/ $\mathrm{H}_{2} \mathrm{O} 9: 1(1.0 \mathrm{~mL})$ for $2 \mathrm{~h}$. The resin was filtered off and the filtrate was added to dry ice-cold $\mathrm{Et}_{2} \mathrm{O}(12.0 \mathrm{~mL})$. After $30 \mathrm{~min}$, the precipitated polyamide was centrifuged, washed once with dry ice-cold $\mathrm{Et}_{2} \mathrm{O}$, centrifuged, the supernatant was discarded and the polyamide pellet was dissolved in $\mathrm{H}_{2} \mathrm{O} / \mathrm{MeCN}$ 1:1 including $0.1 \%$ TFA and freezedried.

Characterization: The freeze-dried products were identified via analytical HPLC-MS on an Agilent 1260 Series HPLC-system (Agilent Technologies) as described before. Electrospray Ionization Mass Spectrometry (ESI-MS) was performed as described before.

\section{Synthesis and Characterization Data of the Polyamides}

Unless otherwise noted, the synthesis of the polyamides was performed manually following the protocol described above. The purity of the polyamides was calculated from the integrated peak areas of the HPLC chromatograms, taken peaks with an $>10 \%$ intensity to the highest peak in the chromatogram and is given as $\%$ for each polyamide.

Im-Py-Py-Im- $\boldsymbol{\gamma}$-Py-Im-Im-Py- $\boldsymbol{\beta}$-COOH (35): After precipitation and lyophilisation, the crude product was obtained from the Fmoc-approach $(35,2.80 \mu \mathrm{mol}, 28 \%)$ in form of a brown solid. The yield was determined spectroscopically. $\boldsymbol{t}_{\boldsymbol{R}}=19.5 \mathrm{~min}$ (gradient 5-75\% B). Purity 93\%. HRMS-ESI $^{+}\left(\mathrm{m} / \mathrm{z}\right.$ ): $[\mathrm{M}+2 \mathrm{H}]^{2+}$ calcd for $\mathrm{C}_{51} \mathrm{H}_{57} \mathrm{~N}_{21} \mathrm{O}_{11} \mathrm{H}_{2}, 570.7346$; found, 570.7359 .
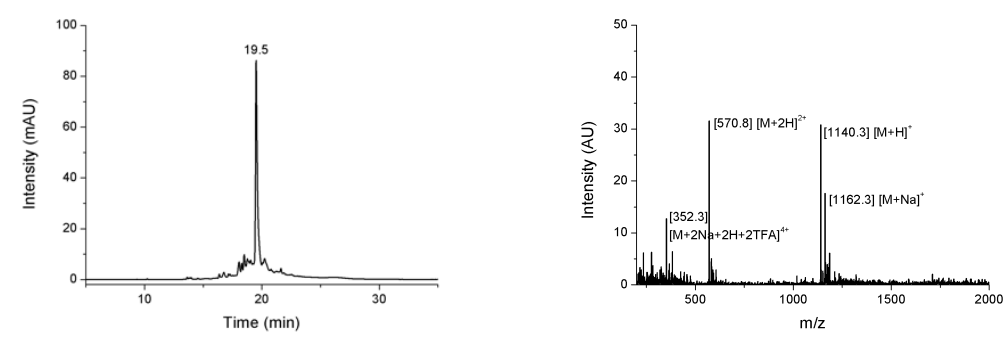

Figure S61: Left: HPLC chromatogram of the crude polyamide hairpin 35 resulting from the Fmoc approach. Gradient 5-75\% B. Right: ESI spectrum of polyamide $\mathbf{3 5}$.

Im-Py-Py-Im- $\boldsymbol{\gamma}$-Py-Im-Im-Py- $\boldsymbol{\beta}$-COOH (35): After precipitation and lyophilisation, the crude product was obtained from the Mtt-approach $(\mathbf{3 5}, 1.42 \mu \mathrm{mol}, 14 \%)$ in form of a brown solid. The yield was determined spectroscopically. $\boldsymbol{t}_{\boldsymbol{R}}=19.5 \mathrm{~min}$ (gradient $5-75 \% \mathrm{~B}$ ). Purity $83 \%$. HRMS-ESI ${ }^{+}(\mathrm{m} / \mathrm{z})$ : $[\mathrm{M}+\mathrm{H}]^{+}$calcd for $\mathrm{C}_{51} \mathrm{H}_{57} \mathrm{~N}_{21} \mathrm{O}_{11} \mathrm{H}, 1140.4625$; found, 1140.4688 . 

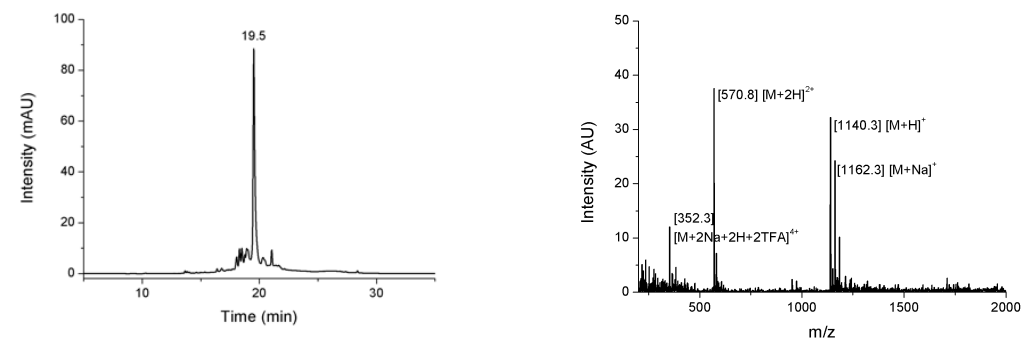

Figure S62: Left: HPLC chromatogram of the crude polyamide hairpin 35 resulting from the Mtt approach. Gradient 5-75\% B. Right: ESI spectrum of polyamide 35.

\section{Synthesis of Im-Py-Py-Im- $\gamma$-Py-Im-Im-Py- $\beta$-Dp (1):}

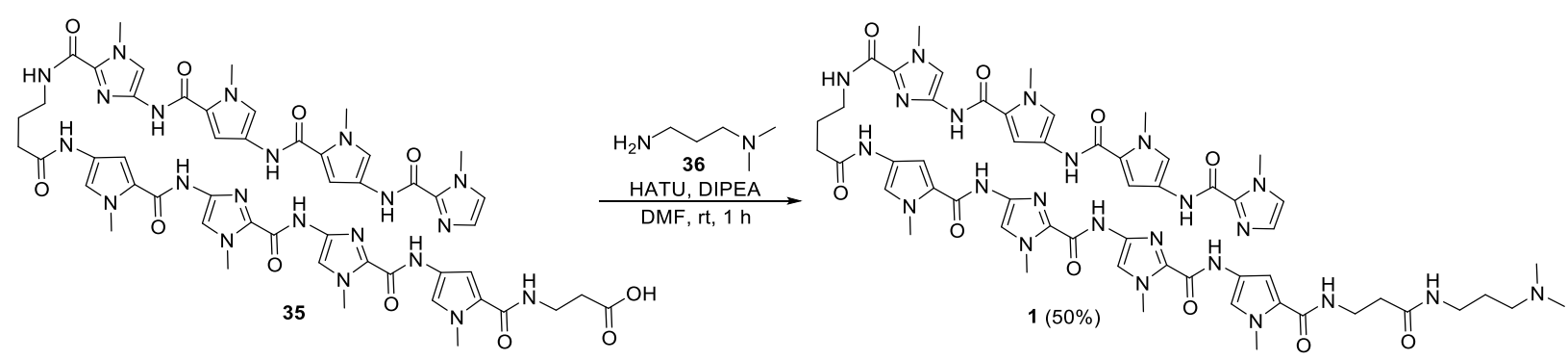

Scheme S5: Reaction scheme for the formation Im-Py-Py-Im- $\gamma$-Py-Im-Im-Py- $\beta$-Dp (1) from Im-Py-Py-Im- $\gamma$-Py-Im-Im-Py- $\beta$ $\mathrm{COOH}(\mathbf{3 5})$.

Im-Py-Py-Im- $\gamma$-Py-Im-Im-Py- $\beta$-COOH (35, $2.2 \mathrm{mg}, 1.93 \mu \mathrm{mol})$ was dissolved in DMF $(10 \mu \mathrm{L})$ and HATU $(2.9 \mathrm{mg}, 7.72 \mu \mathrm{mol})$ and DIPEA $(2.69 \mu \mathrm{L}, 15.4 \mu \mathrm{mol})$ were added. The mixture was shaken for 5 min before $N, N$-dimethyl-1,3-diaminopropane $(0.97 \mu \mathrm{L}, 7.72 \mu \mathrm{mol})$ was added. After shaking for $1 \mathrm{~h}$ at rt, $\mathrm{H}_{2} \mathrm{O} / \mathrm{MeCN}$ $(7: 3,1.0 \mathrm{~mL})$ was added and the solution purified by preparative HPLC as described above. The fractions containing Im-Py-Py-Im- $\gamma$-Py-Im-Im-Py- $\beta$-Dp 1 were lyophilized to give the desired product $(\mathbf{1}, 0.97 \mu \mathrm{mol}$, $50 \%$ ) as pale-brown TFA salt. The yield was determined spectroscopically. $\boldsymbol{t}_{\boldsymbol{R}}=18.7 \mathrm{~min}$ (gradient 5-75\% B ). Purity $>95 \%$. HRMS-ESI ${ }^{+}(m / z)$ : $[\mathrm{M}+2 \mathrm{H}]^{2+}$ calcd for $\mathrm{C}_{56} \mathrm{H}_{69} \mathrm{~N}_{23} \mathrm{O}_{10} \mathrm{H}_{2}, 612.7872$; found, 612.7885 .
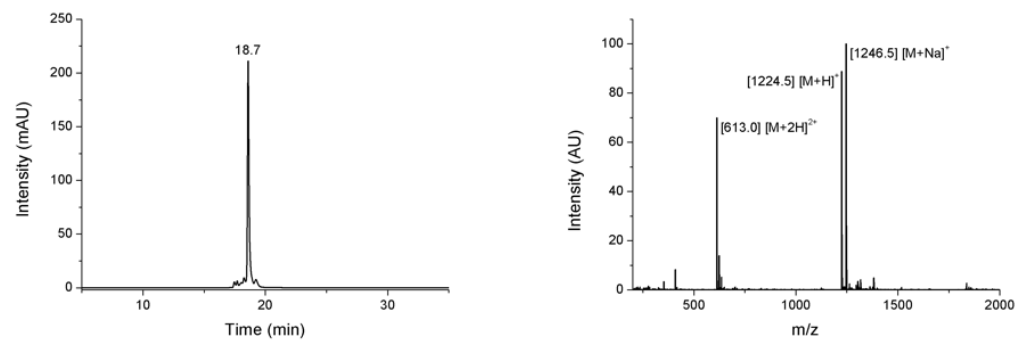

Figure S63: Left: HPLC chromatogram of the purified polyamide hairpin 1. Gradient 5-75\% B. Right: ESI spectrum of polyamide 1. 


\section{Concentration Determination}

Concentrations were measured on a Tecan Spark 20M. Values were background-subtracted.

For the concentration determination of the ssDNAs, the purchased lyophilized DNA was dissolved in milliQ water. Their molar extinction coefficients were determined by using the following formula: ${ }^{\text {[xi] }}$

$\varepsilon_{260 \mathrm{~nm}}=\{(8.8 * \# T)+(7.3 * \# C)+(11.7 * \# G)+(15.4 * \# A)\} * 0.9 * 10^{3} \mathrm{M}^{-1} \mathrm{~cm}^{-1}$

In the formula $\#=$ number of nucleobases determined throughout the DNA sequence, $\mathrm{T}=$ thymine, $\mathrm{C}=$ cytosine, $\mathrm{G}=$ guanine, $\mathrm{A}=$ adenine. Table $\mathrm{S} 1$ summarizes the sequences and the determined extinction coefficients of the ssDNAs.

To form the duplex DNAs, equimolar amounts of the complementary ssDNAs were heated to $95{ }^{\circ} \mathrm{C}$ for $10 \mathrm{~min}$ and slowly cooled down to room temperature. Hybridized duplex DNAs serve the target sequence $\left(\mathrm{dsDNA}_{\mathrm{AGCCGT}}\right)$ and a single mismatch duplex DNA (dsDNA $\left.\mathrm{AGCTGT}\right)$. The mismatch is marked in bold and red.

Table S1. Binding sites, sequences and extinction coefficients of ssDNA, which were used as hybridized duplexes for thermal melting analysis.

\begin{tabular}{lllr}
\hline DNA & binding site & sequence $\left(5^{\prime}, 3^{\prime}\right)$ & $\begin{array}{c}\text { extinction coefficient } \\
\text { at } 260 \mathrm{~nm}\end{array}$ \\
\hline ssDNA $_{A G C C G T}$ & $A G C C G T$ & GGTAGCCGTACC & $111960 \mathrm{M}^{-1} \mathrm{~cm}^{-1}$ \\
ssDNA $_{A C G G C T}$ & $A C G G C T$ & GGTACGGCTACC & $111960 \mathrm{M}^{-1} \mathrm{~cm}^{-1}$ \\
SsDNA $_{A G C T G T}$ & $A G C T G T$ & GGTAGCTGTACC & $113310 \mathrm{M}^{-1} \mathrm{~cm}^{-1}$ \\
ssDNA $_{A C A G C T}$ & $A C A G C T$ & & $115290 \mathrm{M}^{-1} \mathrm{~cm}^{-1}$ \\
\hline
\end{tabular}

For the polyamide hairpin we used an extinction coefficient from the literature: $\varepsilon_{310 \mathrm{~nm}}=69500 \mathrm{M}^{-1} \mathrm{~cm}^{-1[\mathrm{xxii}]}$ 


\section{Thermal Melting Analysis}

Thermal melting analysis was performed for duplex DNAs serving the target sequence of polyamide $\mathbf{1}$ and the target sequence with a single mismatch alone and together with the polyamides. The represented data in table S2 shows the calculated mean from three independent measurements and their corresponding standard deviations. To calculate the melting temperature, the plots of the thermal melting temperature from figure S64 were fitted by applying the Boltzmann equation in the plotting program Origin. The figure shows the corresponding normalized thermal melting curves with their standard deviations.

Table S2. Melting temperatures of DNA hairpins with and without polyamide hairpins. Represented data are mean values and their standard deviation calculated from three independent experiments.

\begin{tabular}{lccc}
\hline DNA & polyamide & $\mathrm{T}_{\mathrm{m}}$ & $\Delta \mathrm{T}_{\mathrm{m}}$ \\
\hline dsDNA & - & $36.2 \pm 0.3^{\circ} \mathrm{C}$ & \\
dsDNACGT $_{\text {AGCCGT }}$ & polyamide $\mathbf{1}$ & $42.8 \pm 0.6{ }^{\circ} \mathrm{C}$ & $6.6{ }^{\circ} \mathrm{C}$ \\
dsDNA $_{\text {AGCTGT }}$ & - & $30.9 \pm 0.2^{\circ} \mathrm{C}$ & \\
dsDNA $_{\text {AGCTGT }}$ & polyamide 1 & $31.9 \pm 0.7^{\circ} \mathrm{C}$ & $1.0{ }^{\circ} \mathrm{C}$ \\
\hline
\end{tabular}

a)

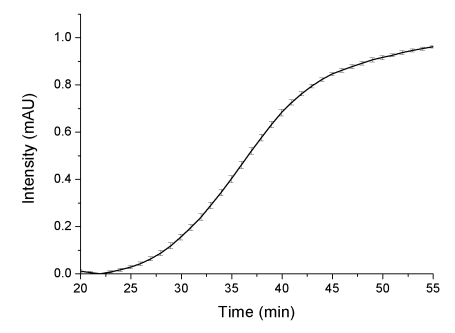

c)

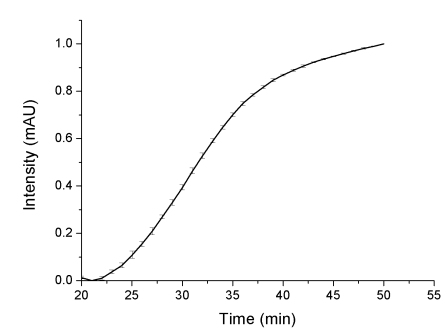

b)

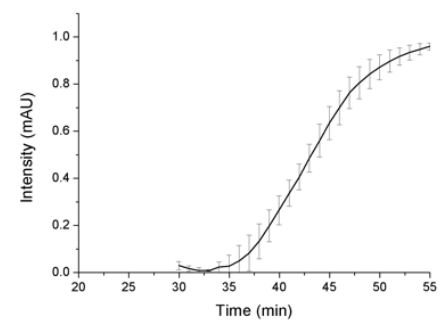

d)

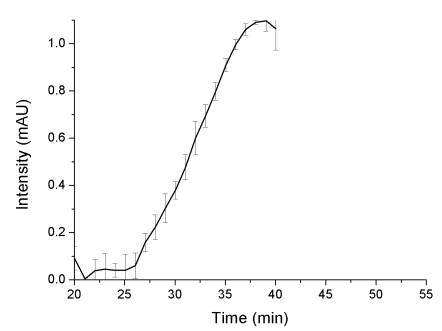

Figure S64: Melting curves of a $1 \mu \mathrm{M}$ duplex dsDNA solution in $10 \mathrm{mM} \mathrm{NaH}_{2} \mathrm{PO}_{4} \mathrm{pH}$ 7.4: 5'-GGTAGCCGTACC-3' without (a) and with (b) addition of $1.2 \mu \mathrm{M}$ polyamide 1 and 5'-GGTAGCTGTACC-3' without (c) and with (d) addition of polyamide $\mathbf{1}$. Represented data is the mean of three independent measurements including their standard deviation. 


\section{Stability of the Mtt-building blocks}

For the study of the stability of the Mtt-building blocks via NMR, a solution of $5 \mathrm{mg}$ of Mtt-Py-OMe (11) or Mtt-Im-OMe (13) was dissolved in $0.6 \mathrm{~mL} \mathrm{CDCl}_{3}$ containing different amounts of TFA to give a $12.6 \mathrm{mM}$ solution. Their ${ }^{1} \mathrm{H}-\mathrm{NMR}$ spectra was measured $3 \mathrm{~min}$ after dissolving the corresponding compound and the chemical shifts of the Mtt-methyl group were compared, as this signal shows the largest difference upon Mtt-deprotection. Figure 2, S65 and S66 show the comparison of the Mtt-lability between protected pyrrole and imidazole. Figure S67 presents the HSQC spectra of Mtt-Py-OMe (11) at 2\% TFA to prove the presence of a methyl group for the new arising peak at higher TFA concentrations.

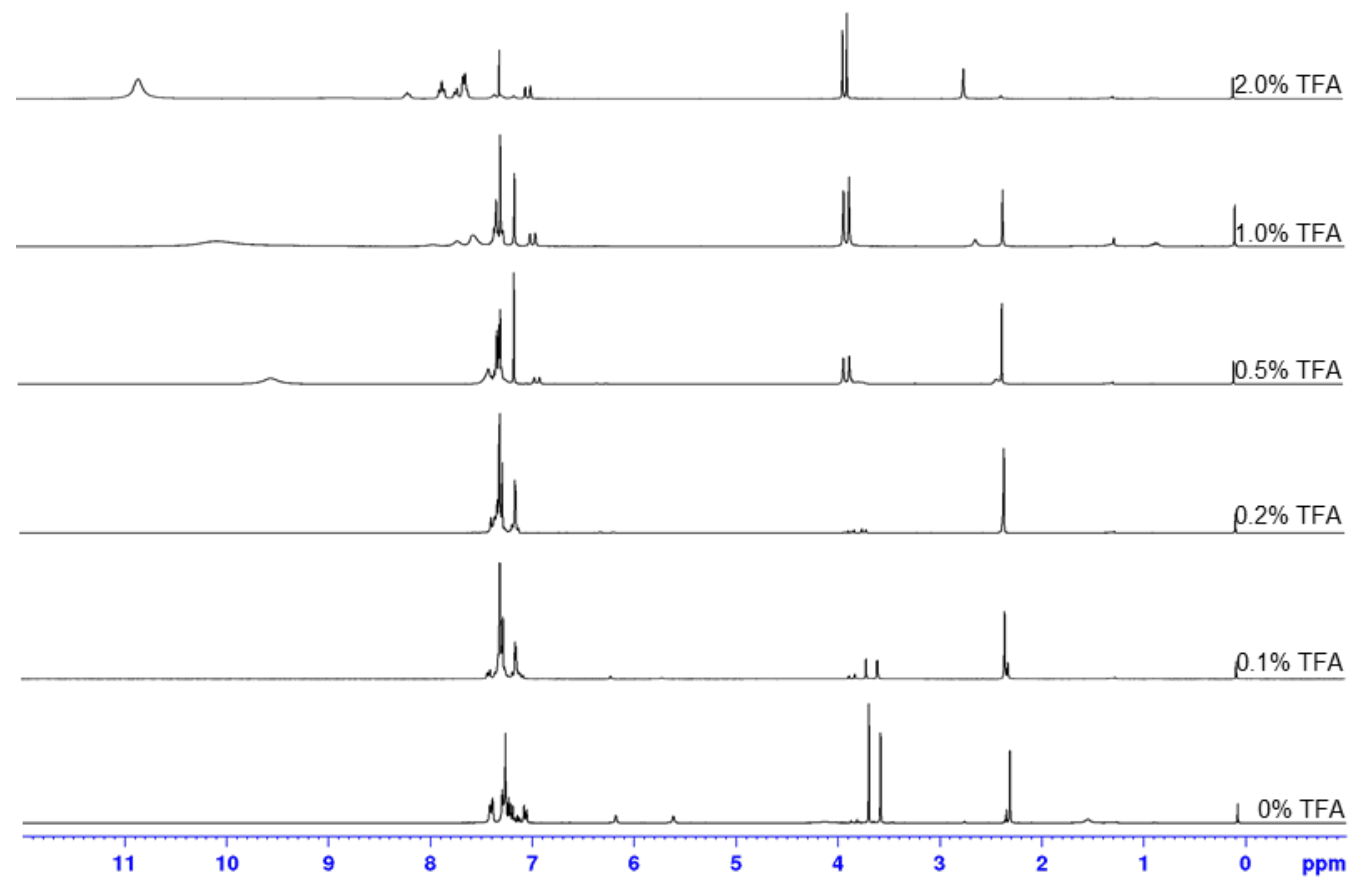

Figure S65: ${ }^{1} \mathrm{H}-\mathrm{NMR}$ spectra (300 MHz) of Mtt-Py-OMe (11) in $\mathrm{CDCl}_{3}$ at different TFA concentrations. 

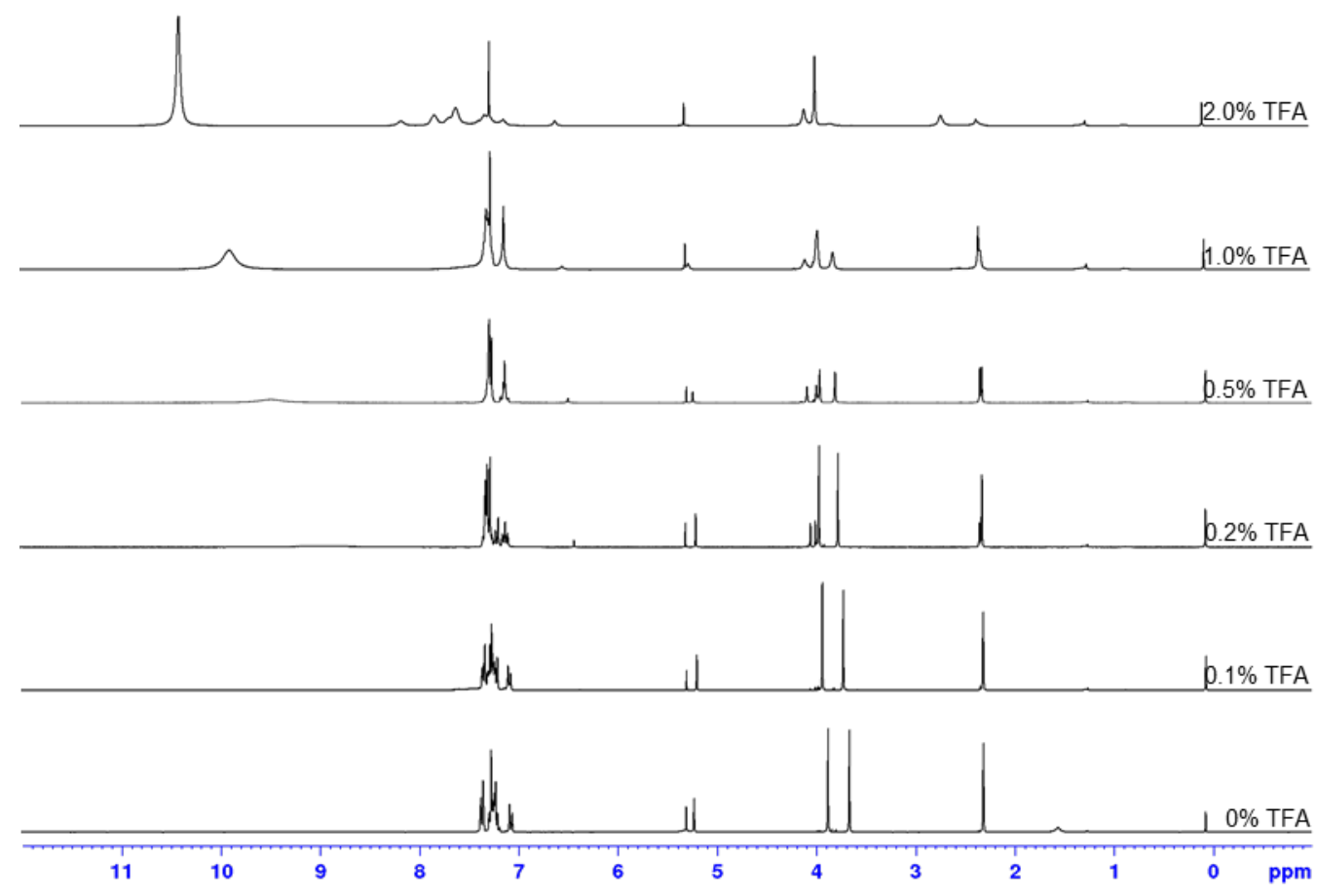

Figure S66: ${ }^{1} \mathrm{H}-\mathrm{NMR}$ spectra (300 MHz) of Mtt-Im-OMe (13) in $\mathrm{CDCl}_{3}$ at different TFA concentrations.

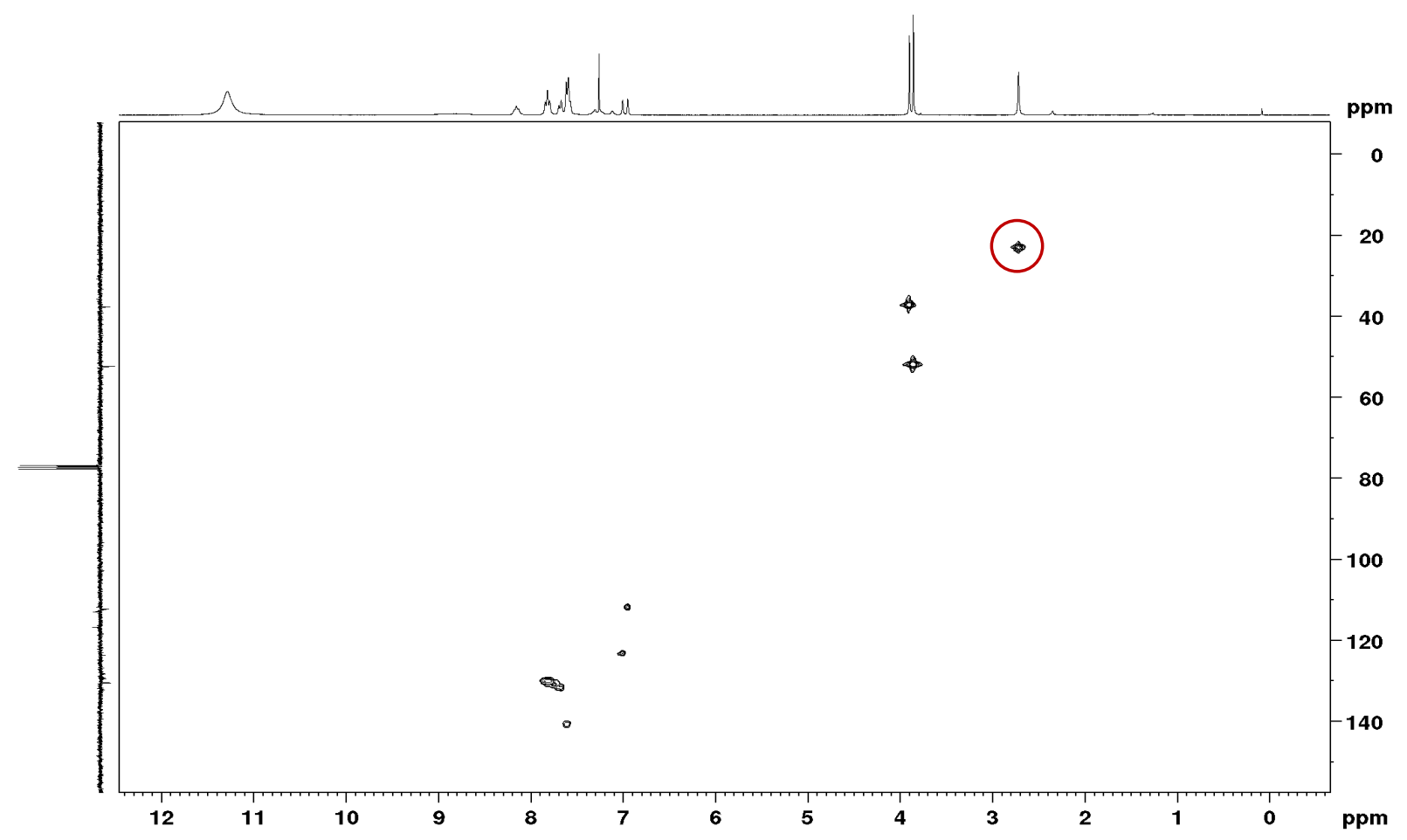

Figure S67: HSQC-spectra (300 MHz, $75 \mathrm{MHz}$ ) of Mtt-Py-OMe (11) in $\mathrm{CDCl}_{3}$ including 2\% TFA. The methyl signal of the shifted Mtt peak is marked with a circle. 
To explain the lowfield shift and the broadening of the signals, the ${ }^{1} \mathrm{H}-\mathrm{NMR}$ spectra of $5 \mathrm{mg} \mathrm{Mtt}-\mathrm{Cl}$ as a reference compound in $0.6 \mathrm{~mL} \mathrm{CDCl} 3$ and increasing amounts of TFA were recorded and are shown in figure S68. Figure S69-S74 show the HSQC, HMBC and APT-spectra of $19 \mathrm{mg} \mathrm{Mtt-Cl}$ in $0.6 \mathrm{~mL} \mathrm{CDCl}_{3}$ with and without addition of $5 \%$ TFA.

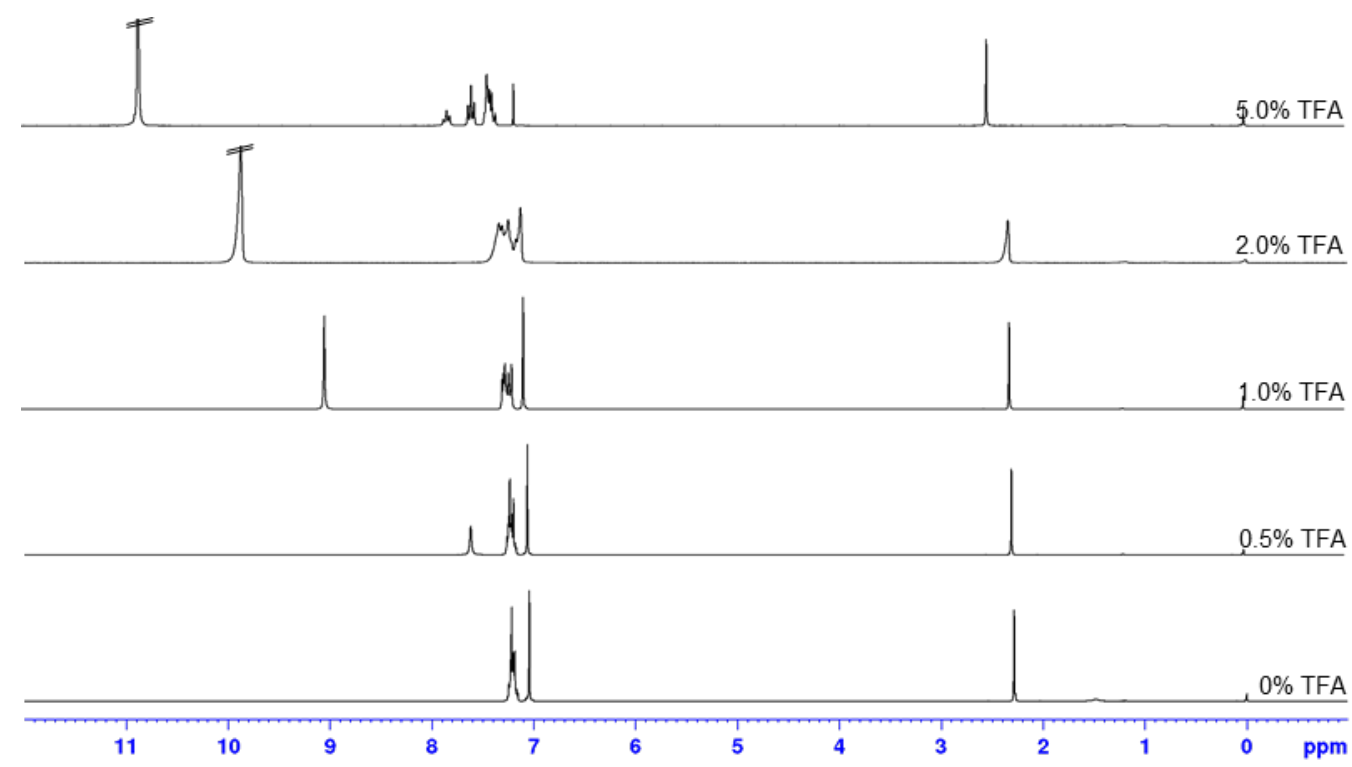

Figure S68: ${ }^{1} \mathrm{H}-\mathrm{NMR}$ spectra $(300 \mathrm{MHz})$ of $\mathrm{Mtt}-\mathrm{Cl}$ in $\mathrm{CDCl}_{3}$ at different TFA concentrations.

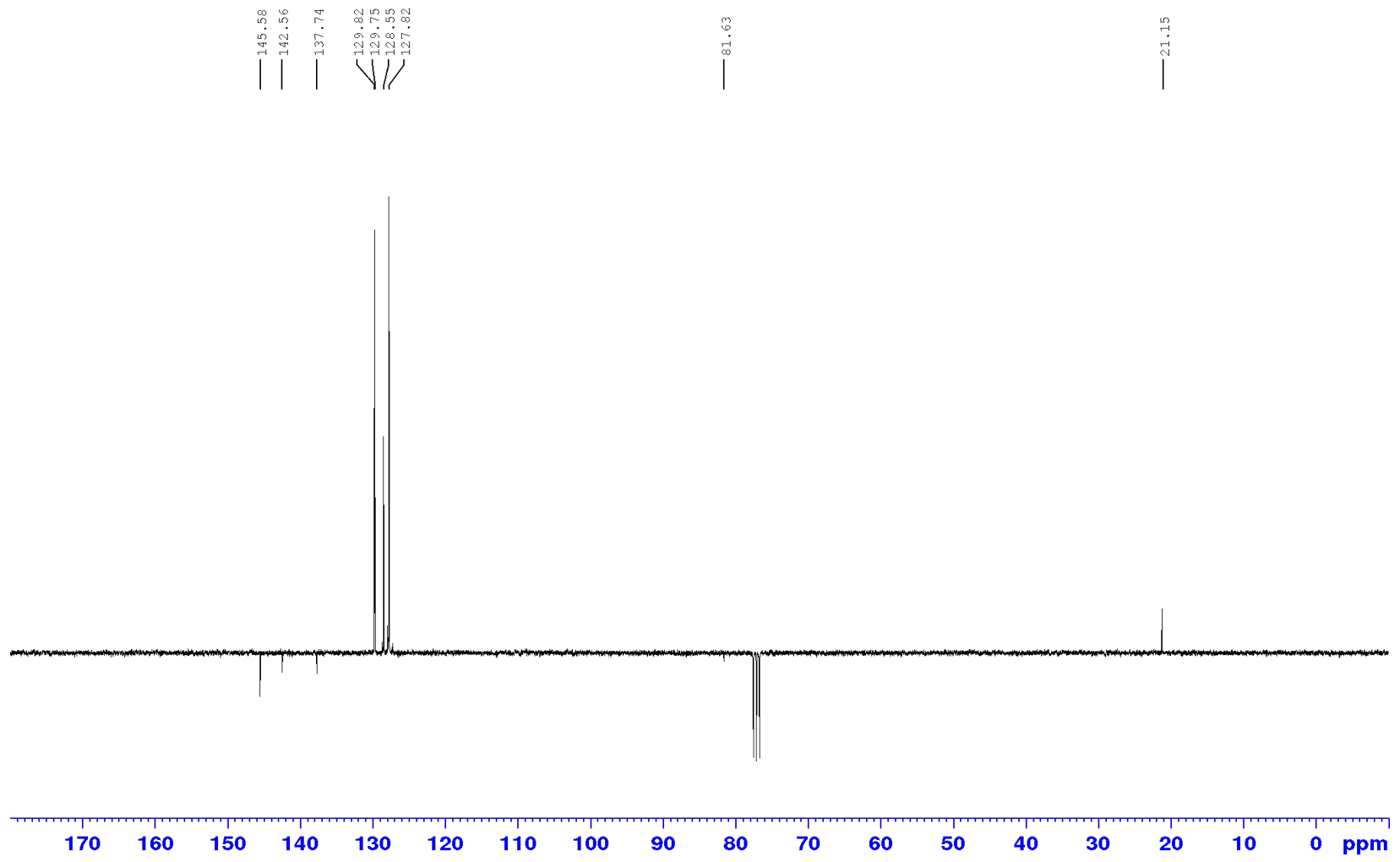

Figure S69: APT-NMR spectra $(300 \mathrm{MHz})$ of $\mathrm{Mtt}-\mathrm{Cl}$ in $\mathrm{CDCl}_{3}$ without addition of TFA. 


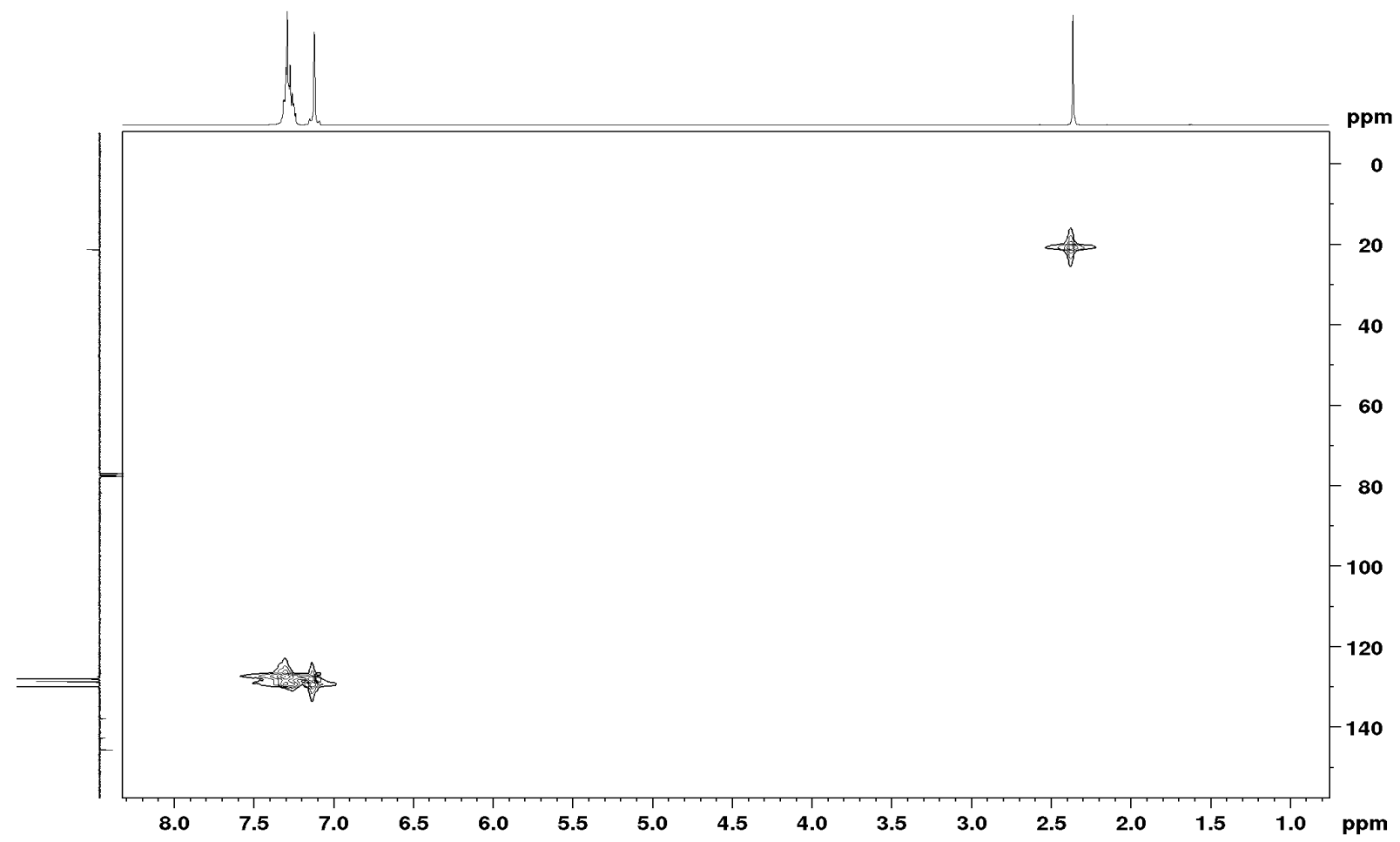

Figure S70: HSQC-NMR spectra $(300 \mathrm{MHz})$ of $\mathrm{Mtt}-\mathrm{Cl}$ in $\mathrm{CDCl}_{3}$ without addition of TFA.

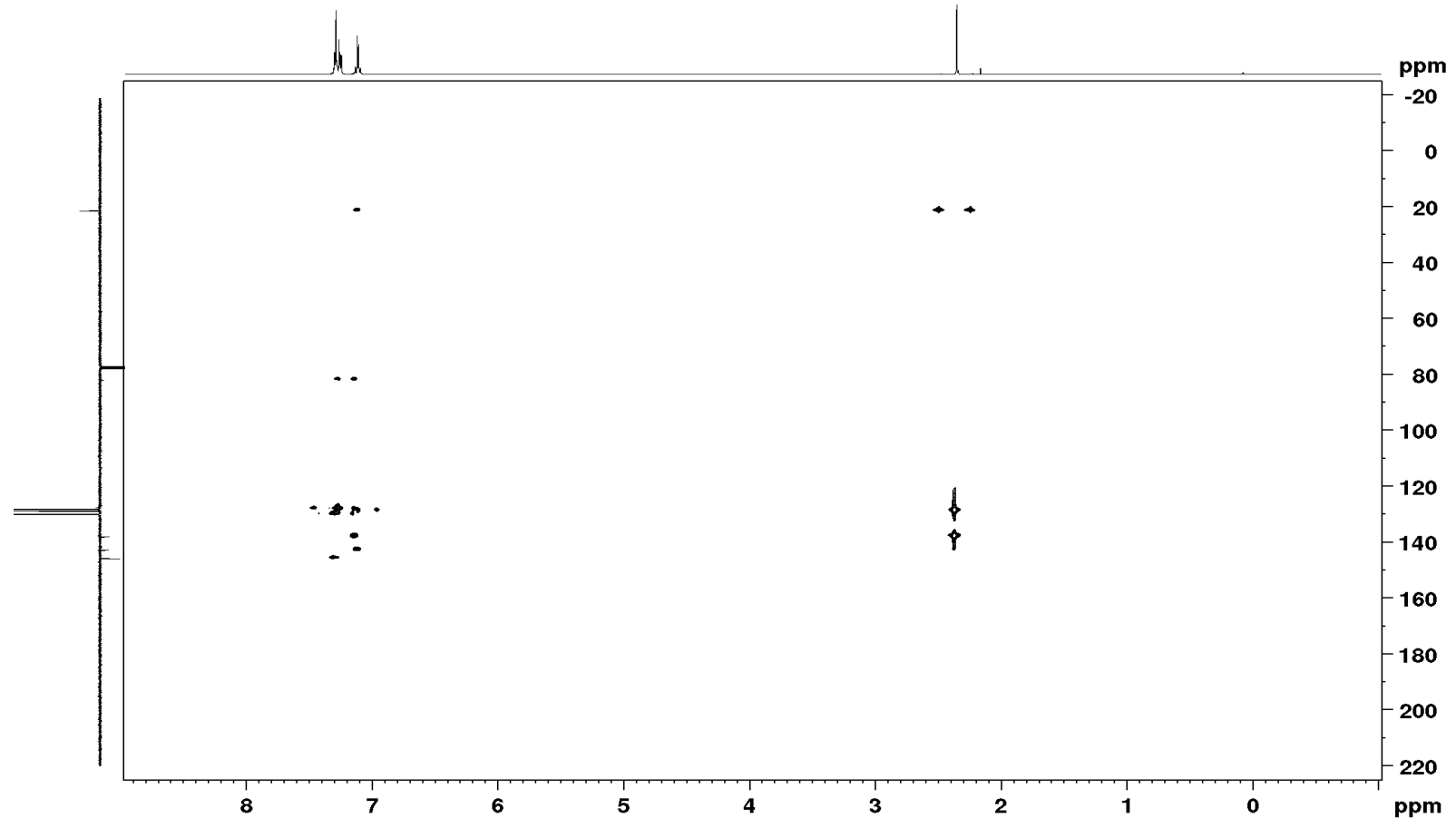

Figure S71: HMBC-NMR spectra (300 MHz) of $\mathrm{Mtt}-\mathrm{Cl}$ in $\mathrm{CDCl}_{3}$ without addition of TFA. 


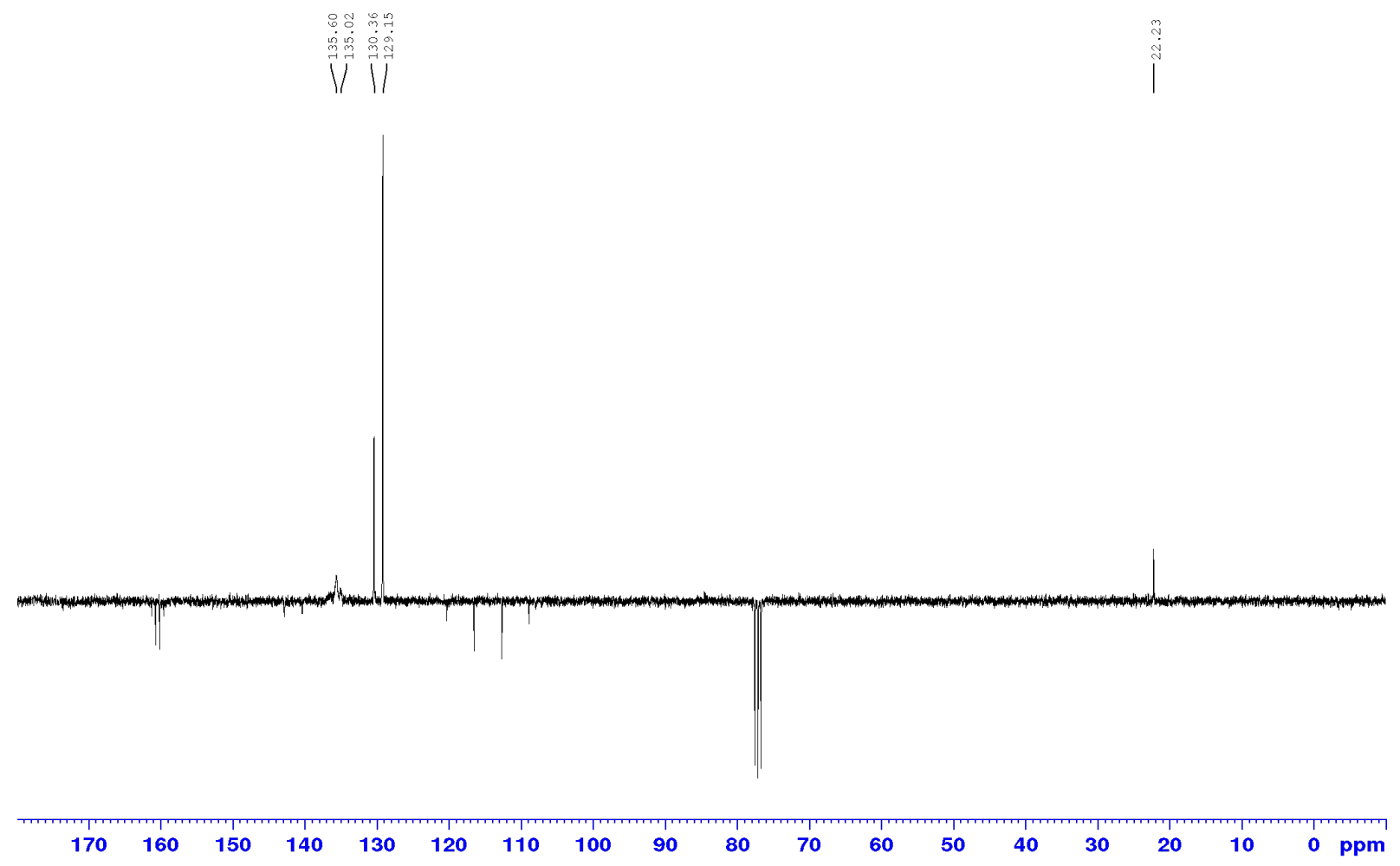

Figure S72: APT-NMR spectra (300 MHz) of $\mathrm{Mtt}-\mathrm{Cl}$ in $\mathrm{CDCl}_{3}$ including 5\% TFA.

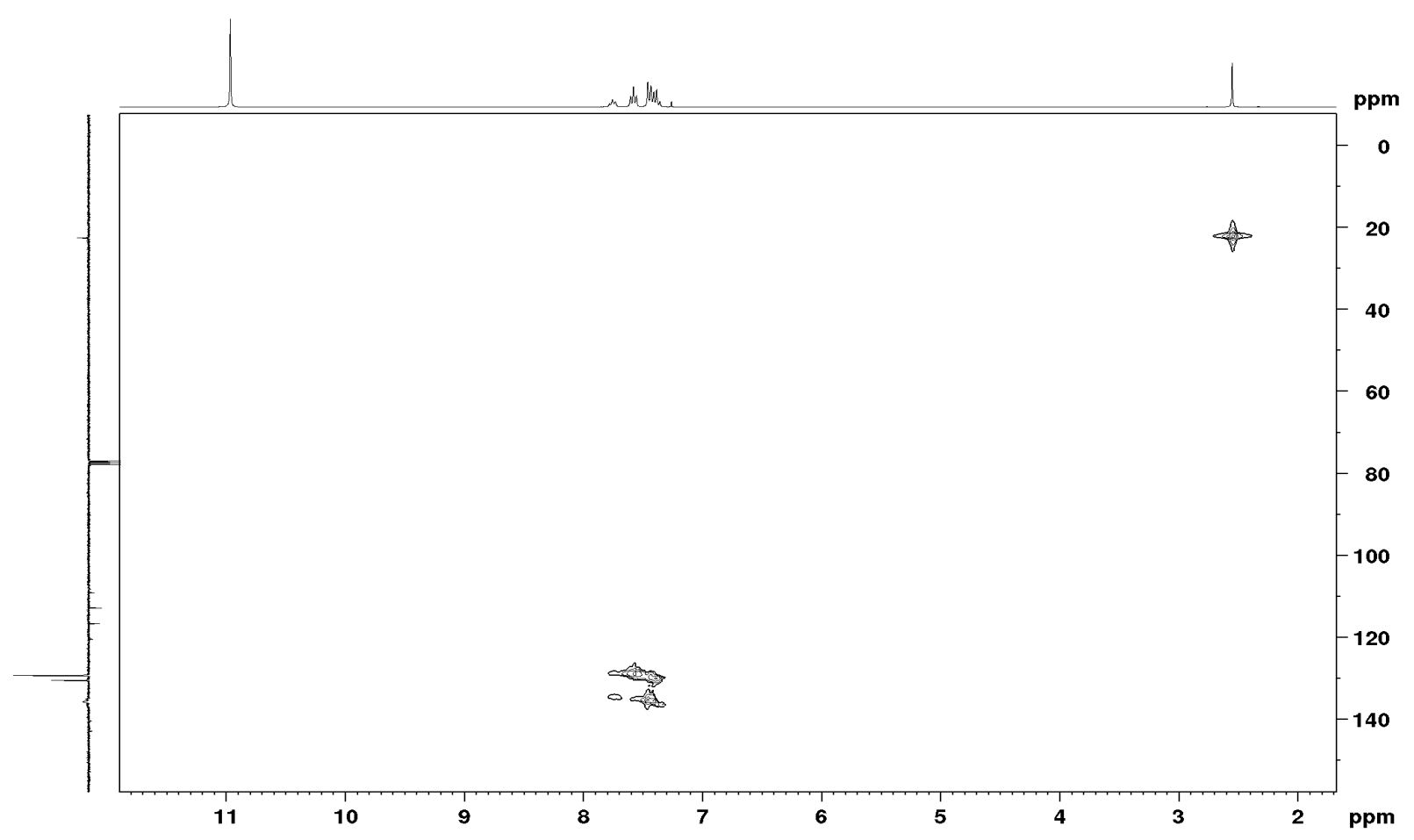

Figure S73: HSQC-NMR spectra (300 MHz) of $\mathrm{Mtt}-\mathrm{Cl}$ in $\mathrm{CDCl}_{3}$ including $5 \% \mathrm{TFA}$. 


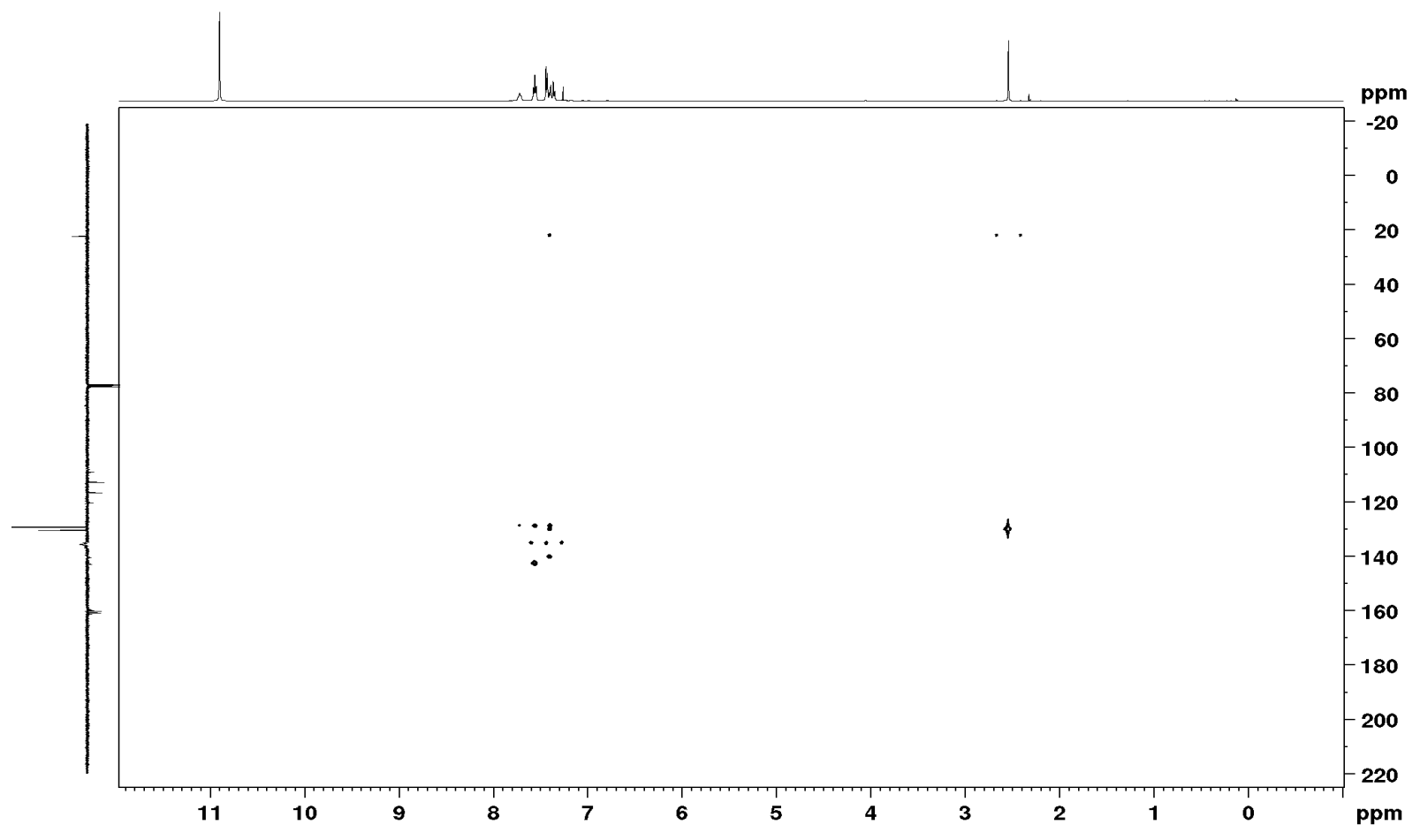

Figure S74: HMBC-NMR spectra (300 MHz) of $\mathrm{Mtt}-\mathrm{Cl}$ in $\mathrm{CDCl}_{3}$ including $5 \%$ TFA.

Figure S75 represents the APT-spectra of a 5\% TFA solution in $\mathrm{CDCl}_{3}$.
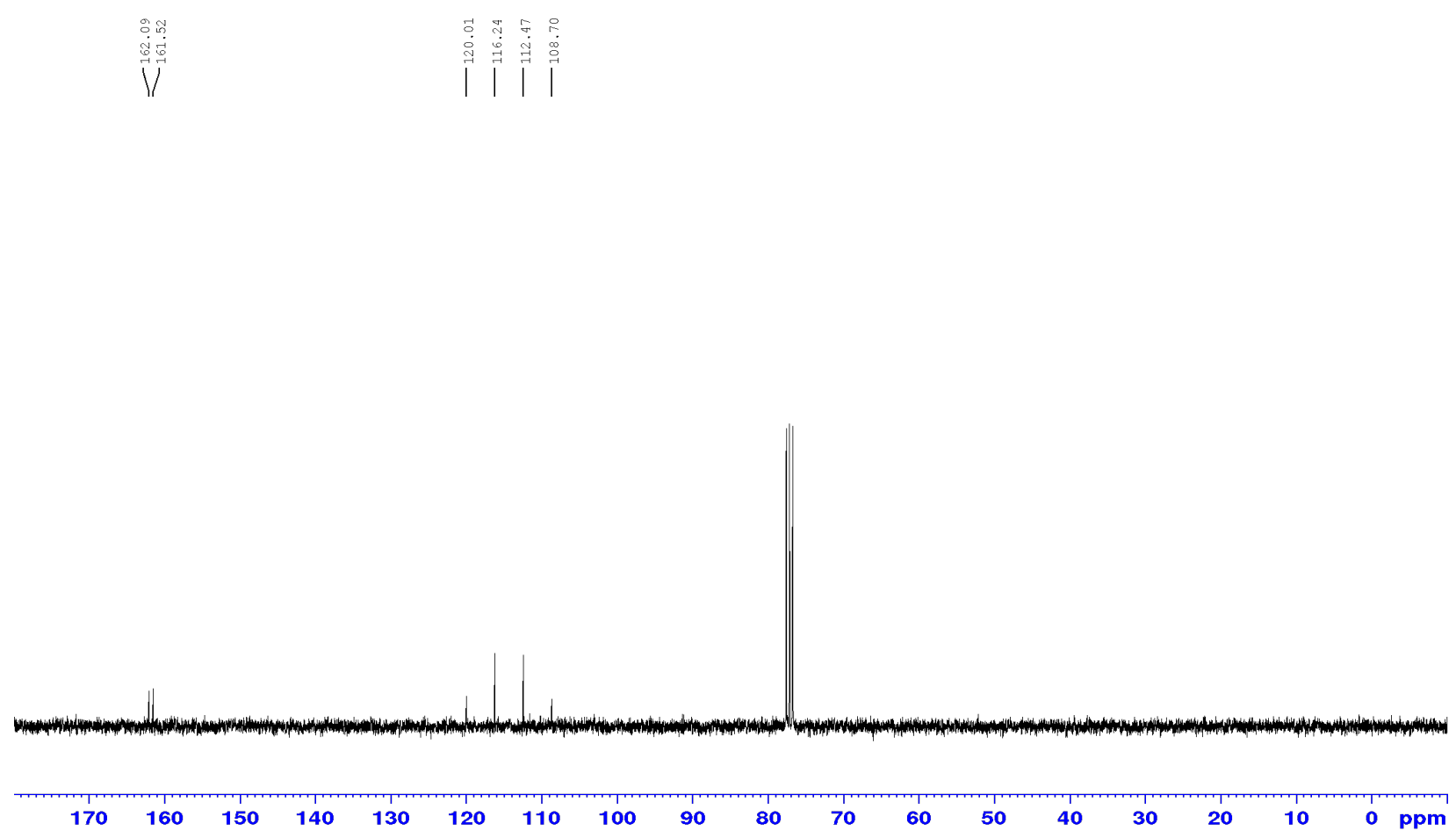

Figure S75: APT-NMR spectra $(300 \mathrm{MHz})$ of $5 \%$ TFA in $\mathrm{CDCl}_{3}$. 


\section{Additional Experiments}

Further experiments were performed to prove the formation of Mtt-butyrolactam (36) and optimize the coupling conditions to implement $\mathrm{Mtt}-\gamma-\mathrm{OH}$ (5).

\section{Synthesis of Mtt-butyrolactam (36)}

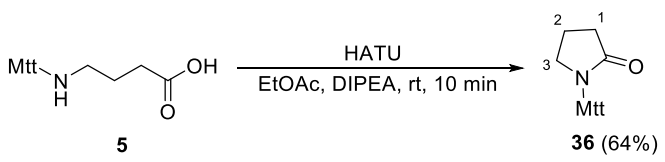

Scheme S6: Reaction scheme for the formation of Mtt-butyrolactam (36) from Mtt- $\gamma-\mathrm{OH}(5)$.

1-(diphenyl(p-tolyl)methyl)pyrrolidin-2-one (36): Mtt- $\gamma$-OH $(50.0 \mathrm{mg}, 139 \mathrm{nmol})$ and HATU (52.9 mg, $139 \mathrm{nmol})$ were dissolved in EtOAc $(500 \mu \mathrm{L})$ and DIPEA $(73 \mu \mathrm{L}, 419 \mathrm{nmol})$ added. After $10 \mathrm{~min}$ at $\mathrm{rt}$, the solvent was removed under reduced pressure and the crude purified by column chromatography (silica, $\mathrm{CH}_{2} \mathrm{Cl}_{2} / \mathrm{NEt}_{3}$ 100:1 and $n$-pentane/EtOAc 2:1) to yield the desired product (36, $30.2 \mathrm{mg}, 88.4 \mathrm{nmol}, 64 \%$ ) as colourless solid. $\mathbf{R}_{\boldsymbol{f}}=0.42$ (n-pentane/EtOAc 1:1). ${ }^{1} \mathbf{H}-\mathbf{N M R}\left(300 \mathrm{MHz}, \mathrm{CDCl}_{3}, \delta\right): 7.30-7.17(\mathrm{~m}, 10 \mathrm{H}$, $\left.\mathrm{CH}_{\text {arom. }}\right), 7.16-7.06$ (m, 4H, CHarom.), $3.46\left(\mathrm{t}, 2 \mathrm{H}, J=6.8 \mathrm{~Hz}, \mathrm{CH}_{2}-3\right), 2.37\left(\mathrm{t}, 2 \mathrm{H}, J=8.0 \mathrm{~Hz}, \mathrm{CH}_{2}-1\right), 2.30$ (s, 3H, CH3-Mtt), $2.11-1.97\left(\mathrm{~m}, 2 \mathrm{H}, \mathrm{CH}_{2}-2\right) .{ }^{13} \mathbf{C}-\mathbf{N M R}\left(75 \mathrm{MHz}, \mathrm{CDCl}_{3}, \delta\right): 178.2(\mathrm{C}=\mathrm{O}), 144.3\left(2 \mathrm{C}, \mathrm{C}_{\mathrm{q}}\right)$, $141.3\left(\mathrm{C}_{\mathrm{q}}\right), 137.8\left(\mathrm{C}_{\mathrm{q}}\right), 130.8(2 \mathrm{C}, \mathrm{CH}), 130.7(4 \mathrm{C}, \mathrm{CH}), 129.1(2 \mathrm{C}, \mathrm{CH}), 128.5(4 \mathrm{C}, \mathrm{CH}), 127.8(2 \mathrm{C}, \mathrm{CH})$, $75.0\left(\mathrm{C}_{\mathrm{q}}\right)$, $51.6\left(\mathrm{CH}_{2}\right), 34.2\left(\mathrm{CH}_{2}\right), 20.9\left(\mathrm{CH}_{3}\right), 19.8\left(\mathrm{CH}_{2}\right)$. HRMS-ESI ${ }^{+}(\mathrm{m} / \mathrm{z}):[\mathrm{M}+\mathrm{H}]^{+}$calcd for $\mathrm{C}_{24} \mathrm{H}_{23} \mathrm{NOH}, 342.1852$; found, 342.1862. FT-IR (neat) $\tilde{v}=3020(\mathrm{w}), 2966(\mathrm{w}), 2874(\mathrm{w}), 1692(\mathrm{~s}), 1509$ (w), $1491(\mathrm{w}), 1443(\mathrm{w}), 1389(\mathrm{~m}), 1319(\mathrm{w}), 1282(\mathrm{w}), 1259(\mathrm{~m}), 1190(\mathrm{w}), 1159(\mathrm{w}), 1121(\mathrm{w}), 1086(\mathrm{w})$, $1022(\mathrm{w}), 945(\mathrm{w}), 929(\mathrm{w}), 906(\mathrm{w}), 804(\mathrm{~m}), 761(\mathrm{w}), 740(\mathrm{~m}), 698(\mathrm{~s}), 664(\mathrm{w}), 635(\mathrm{w}), 581(\mathrm{w}), 546$ (m), $519(\mathrm{w}), 498(\mathrm{w}), 482(\mathrm{w})$. 


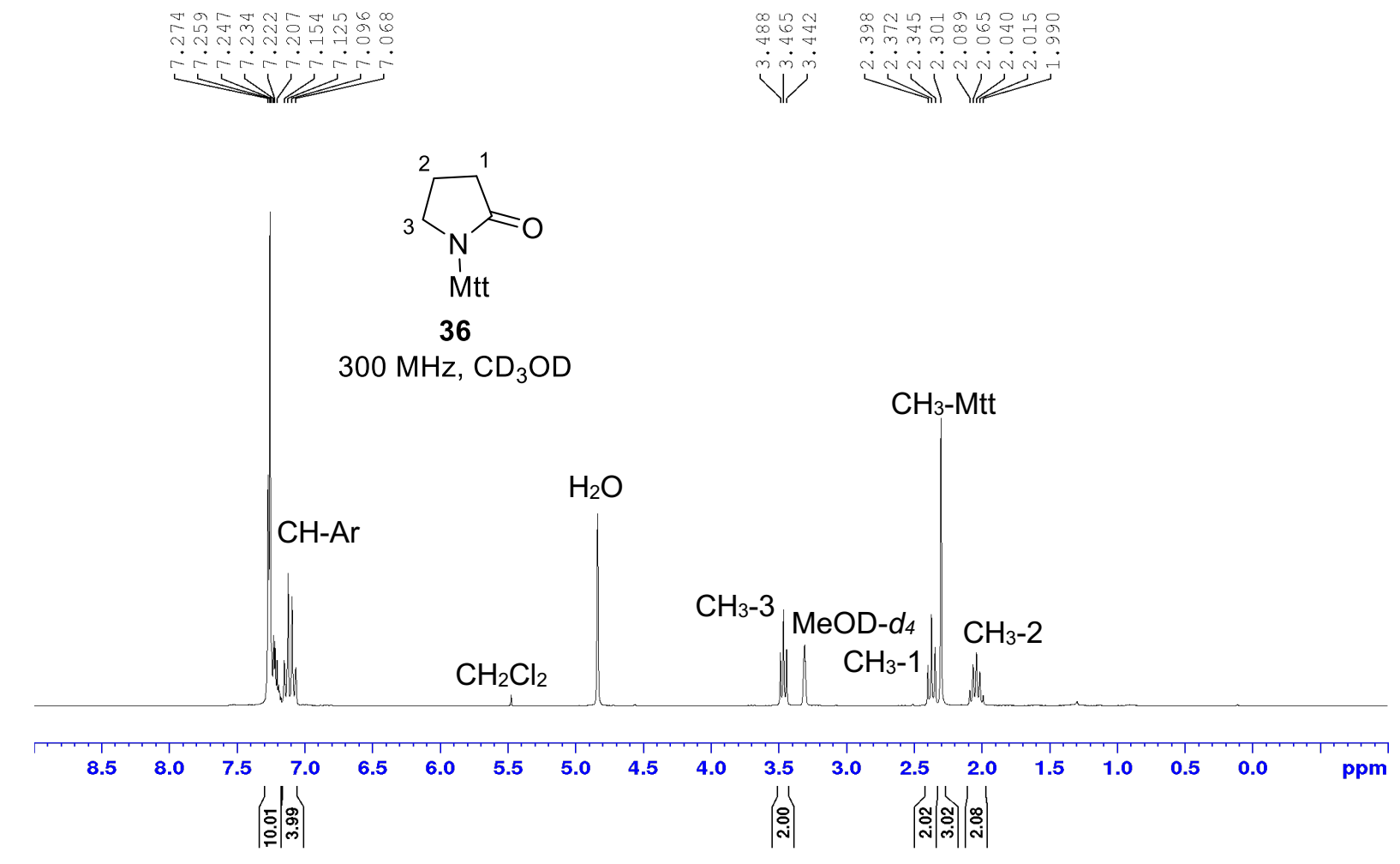

Figure S76: ${ }^{1} \mathrm{H}-\mathrm{NMR}(300 \mathrm{MHz}$ ) spectrum of Mtt-butyrolactam (36).
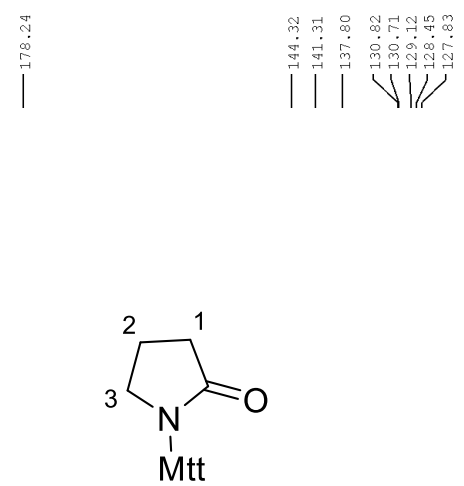

36

$75 \mathrm{MHz}, \mathrm{CD}_{3} \mathrm{OD}$

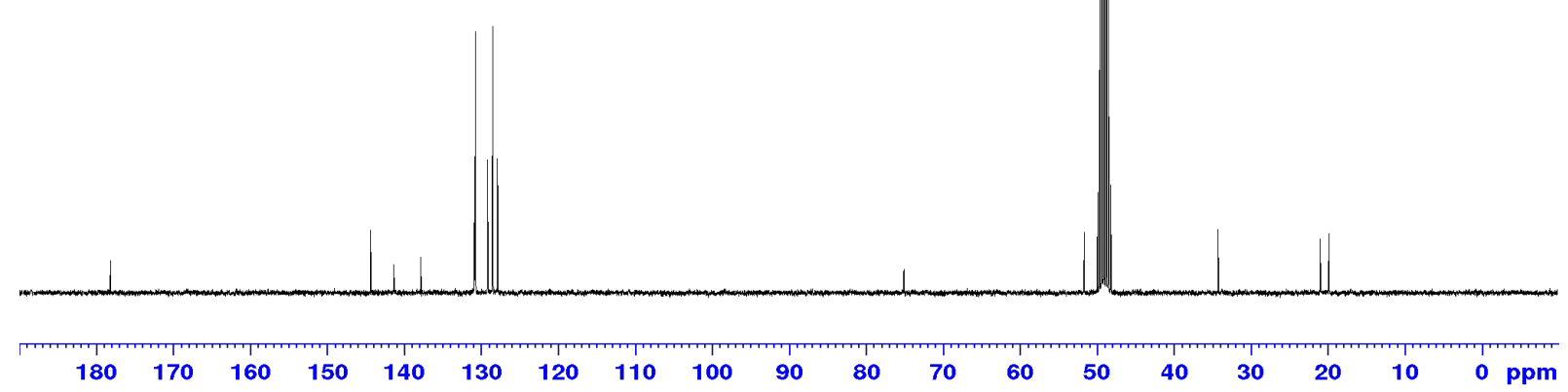

Figure S77: ${ }^{1} \mathrm{H}-\mathrm{NMR}(75 \mathrm{MHz})$ spectrum of Mtt-butyrolactam (36). 


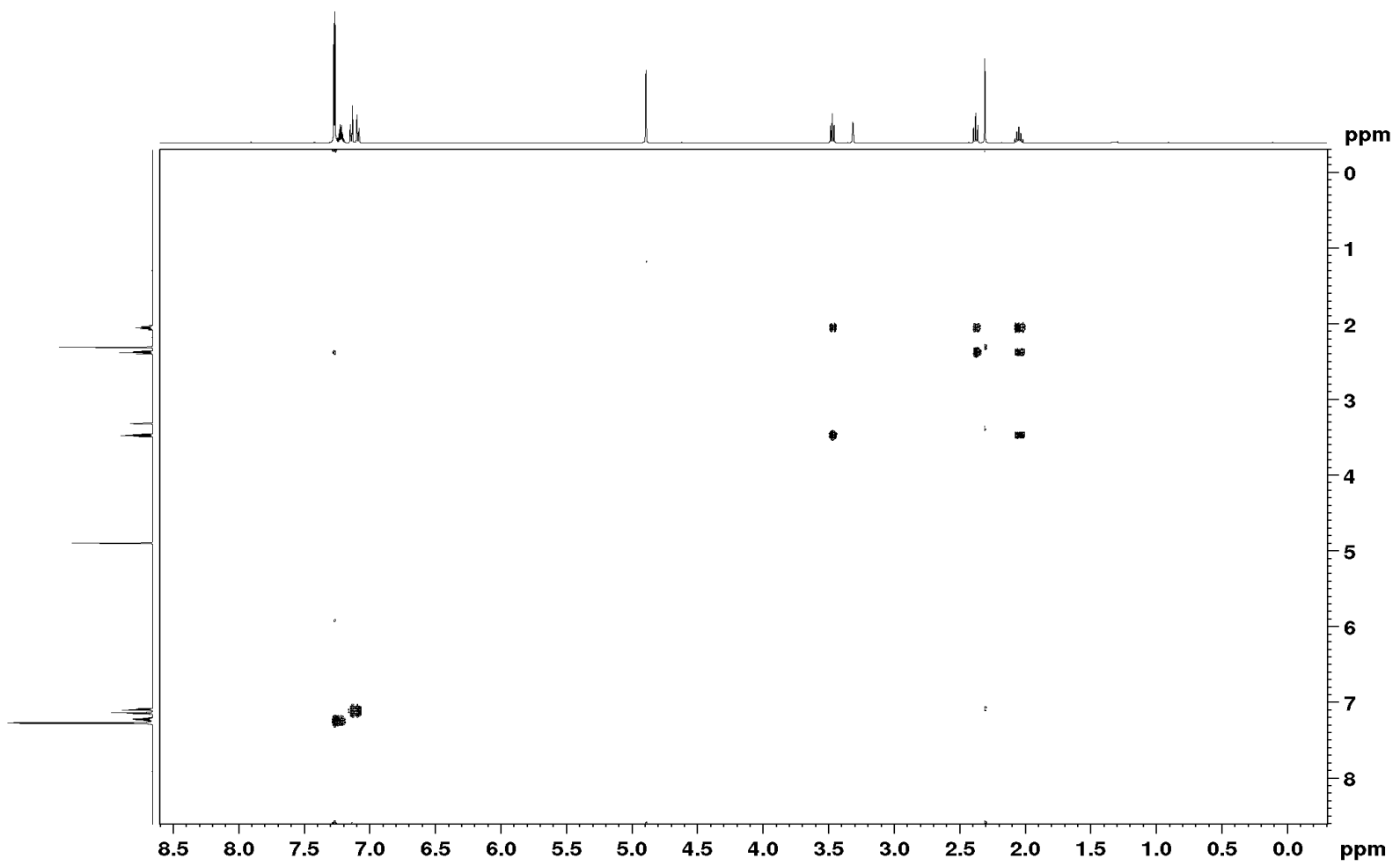

Figure S78: COSY-NMR (500 MHz) spectrum of Mtt-butyrolactam (36).

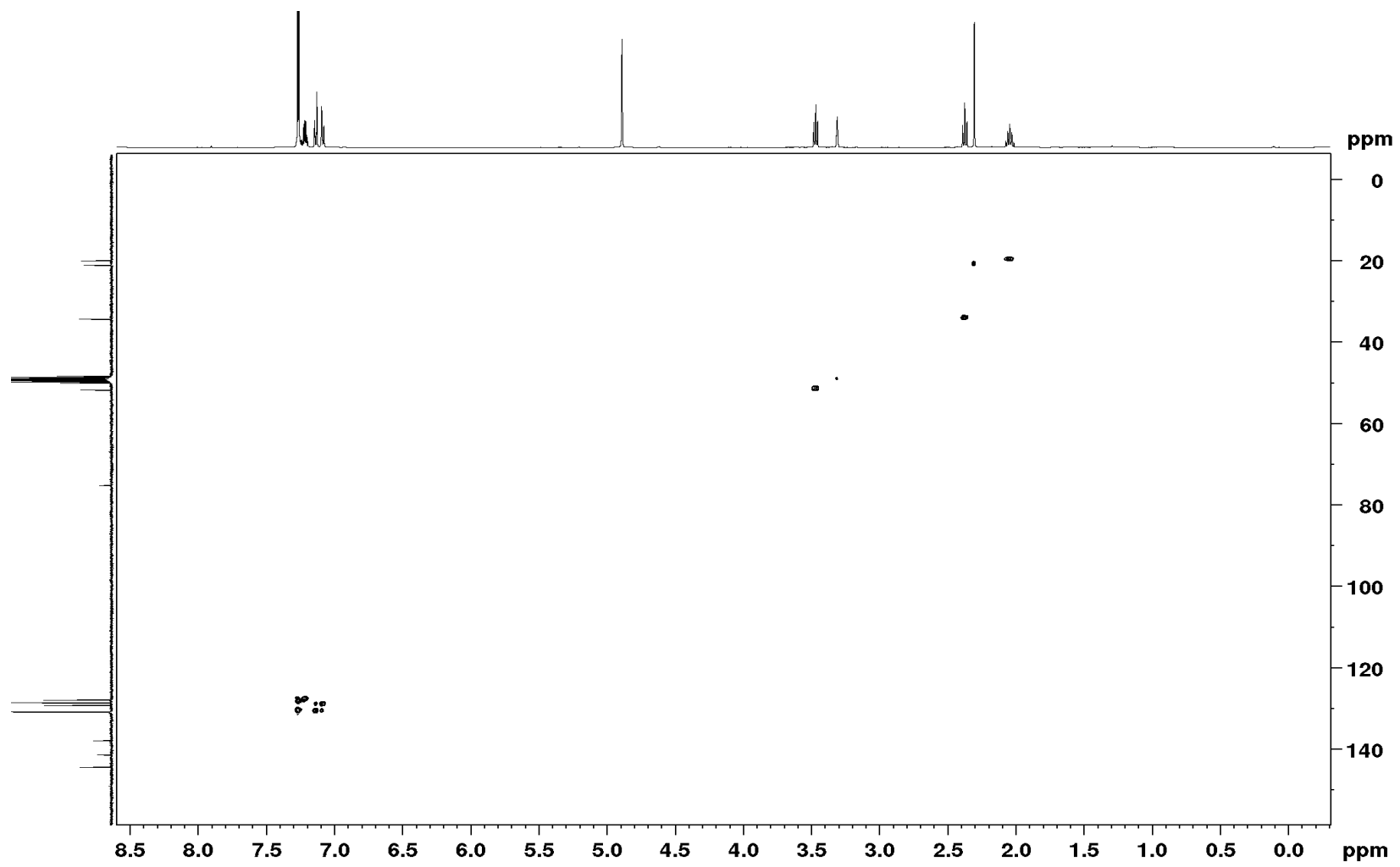

Figure S79: HSQC-NMR (500 MHz, $75 \mathrm{MHz}$ ) spectrum of Mtt-butyrolactam (36). 


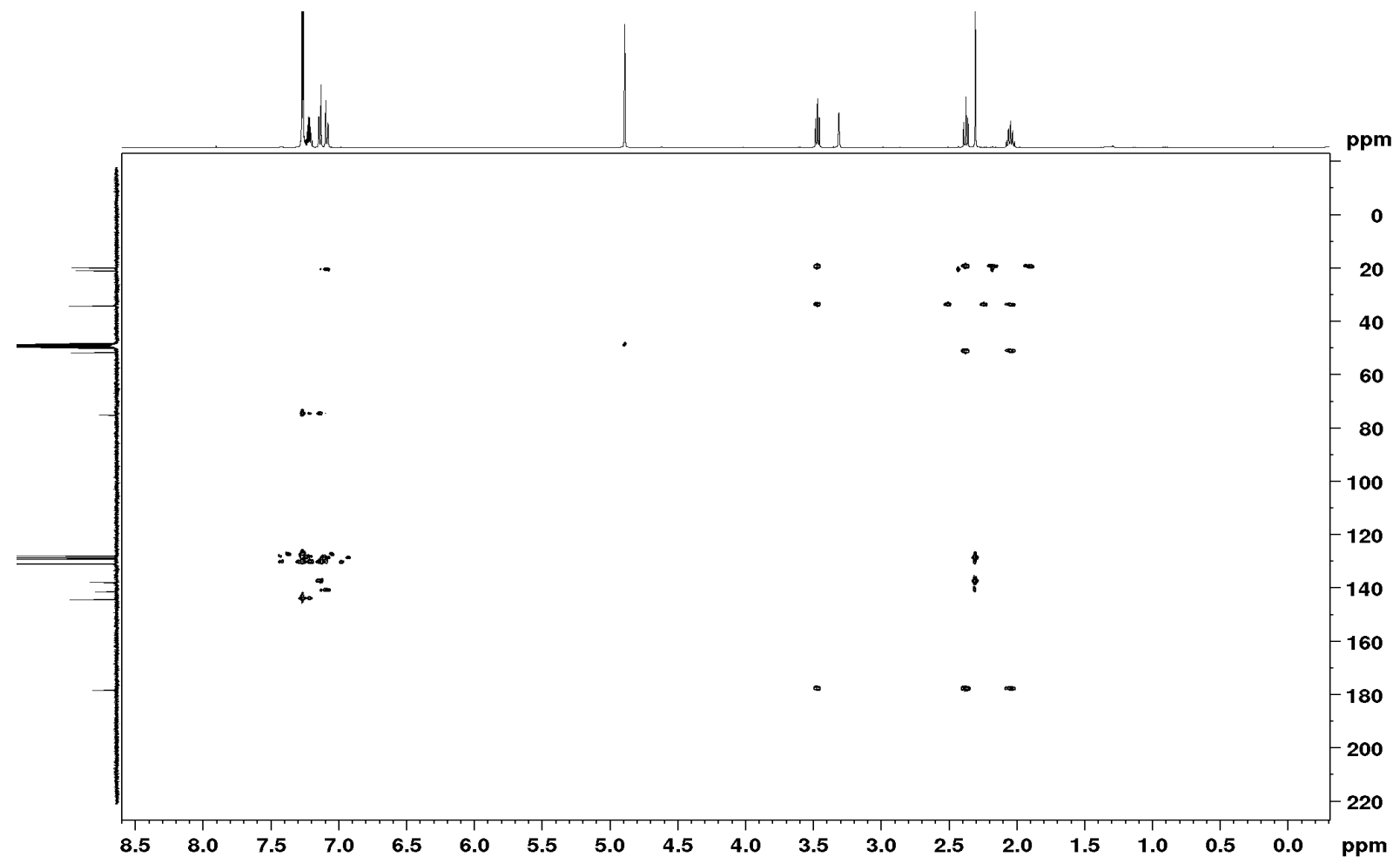

Figure S80: HMBC-NMR (500 MHz, $75 \mathrm{MHz}$ ) spectrum of Mtt-butyrolactam (36).

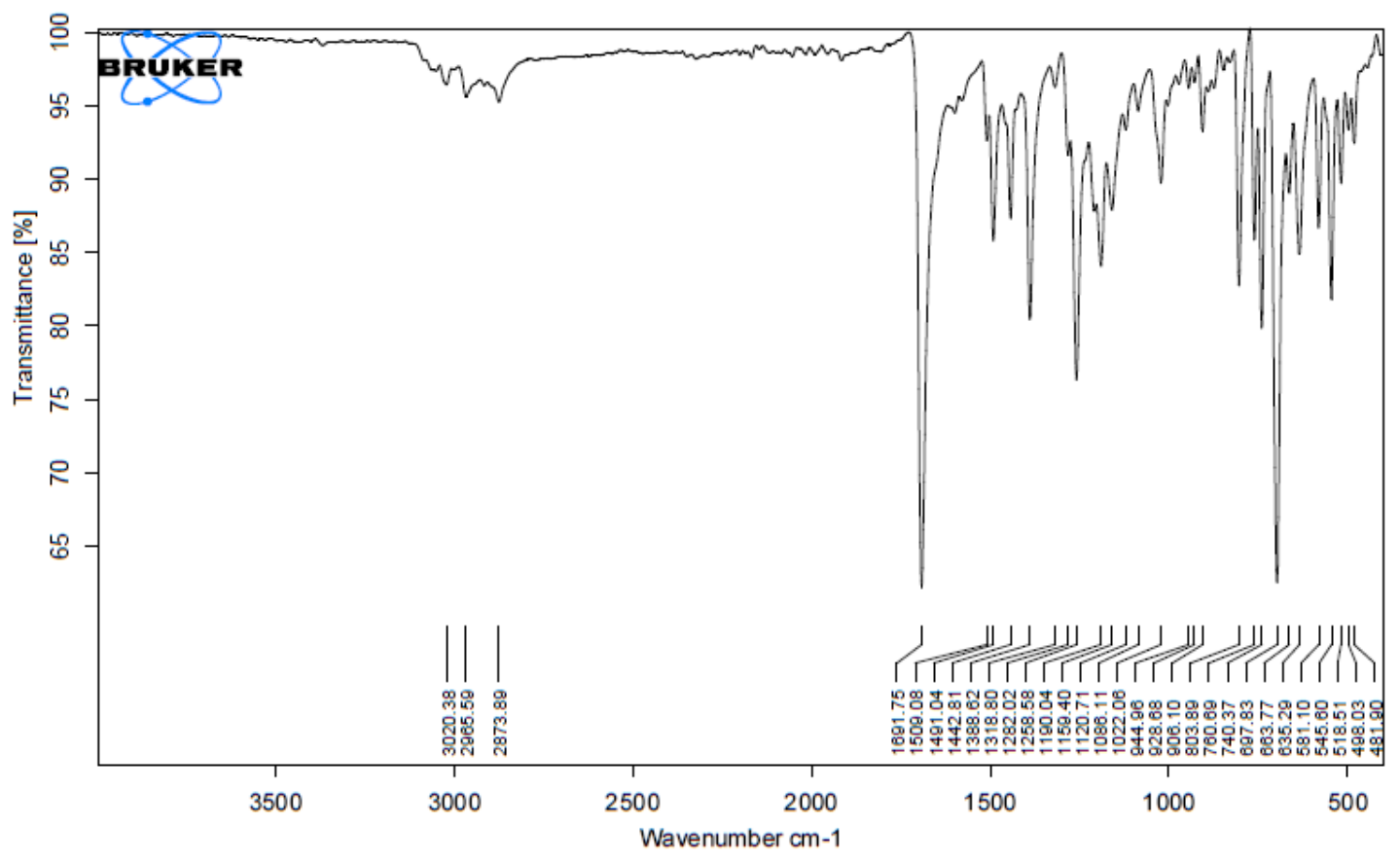

Figure S81: IR spectrum of Mtt-butyrolactam (36). 


\section{Test Implementation of Mtt- $\gamma-\mathrm{OH}$}

For the introduction of Mtt- $\gamma-\mathrm{OH}$, Mtt-Py-Im-Im-Py- $\beta$ was synthesized on solid support. Figure S82 shows the HPLC chromatogram of the successful coupling with a purity $>95 \%$.
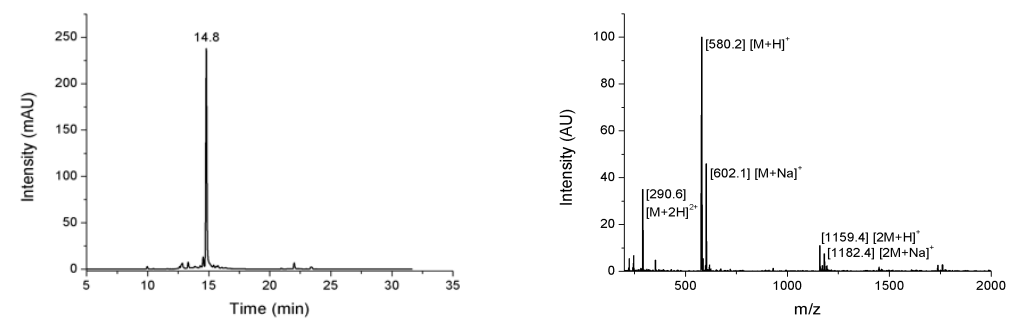

Figure S82: Left: HPLC chromatogram of the test cleavage of $\mathrm{H}_{2} \mathrm{~N}-\mathrm{Py}-\mathrm{Im}-\mathrm{Im}-\mathrm{Py}-\beta-\mathrm{COOH}$. Gradient 5-95\% B. Right: ESI spectrum of $\mathrm{H}_{2} \mathrm{~N}-\mathrm{Py}-\mathrm{Im}-\mathrm{Im}-\mathrm{Py}-\beta-\mathrm{COOH}$.

In $3 \mu \mathrm{mol}$ scales, the Mtt group was deprotected as described above and $\mathrm{Mtt}-\gamma-\mathrm{OH}(\mathbf{5})$ coupled at $0.3 \mathrm{M}$ in NMP with different coupling conditions over one hour. After washing, capping and cleavage, HPLC analysis revealed the coupling efficiency. Integration of the product peak $\left(\mathrm{H}_{2} \mathrm{~N}-\gamma-\mathrm{Py}-\mathrm{Im}-\mathrm{Im}-\mathrm{Py}-\beta-\mathrm{COOH}\right.$, $t_{R}=17.3 \mathrm{~min}, 665.2 \mathrm{~g} / \mathrm{mol}$ ) in comparison to peaks with a $>10 \%$ intensity to the highest peak in the chromatogram let to the described coupling yields. The peak at $18.8 \mathrm{~min}$ corresponds to the acetylated starting material with a mass of $622.2 \mathrm{~g} / \mathrm{mol}$. The corresponding chromatograms are shown in figure S83.

a)

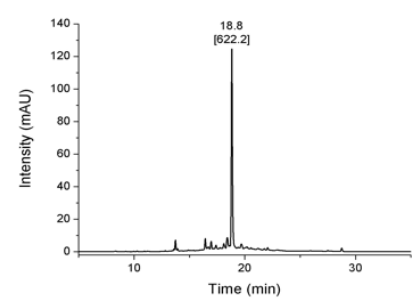

d)

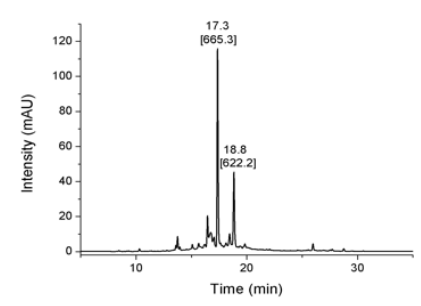

g)

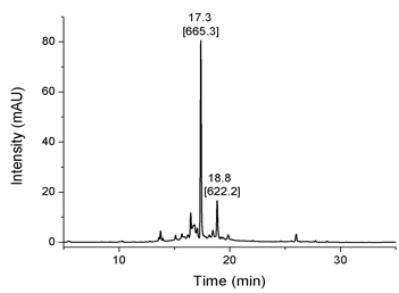

b)

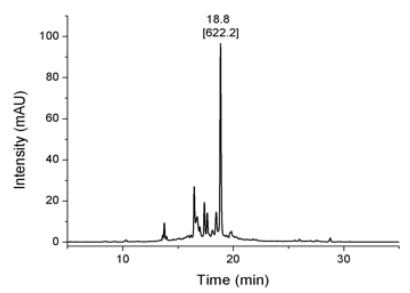

e)

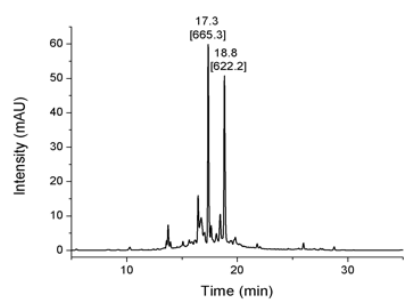

c)

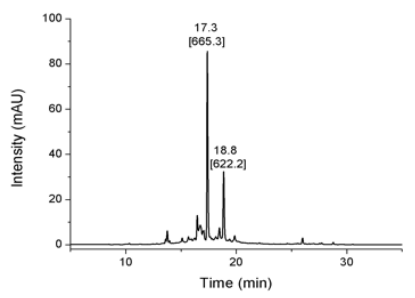

f)

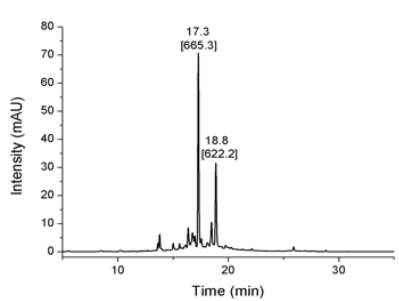

Figure S83: HPLC chromatograms of the test couplings for the introduction of Mtt- $\gamma-\mathrm{OH}$ (5). Gradient 5-75\% B. Coupling conditions were a) 4 eq Mtt- $\gamma-\mathrm{OH}, 4$ eq HATU, 12 eq DIPEA; b) 4 eq Mtt- $\gamma-\mathrm{OH}, 4$ eq HATU, 12 eq NMM; c) 4 eq Mtt- $\gamma$-OH, 4 eq HATU, 12 eq pyridine; d) 4 eq Mtt- $\gamma-\mathrm{OH}, 4$ eq HATU, 8 eq pyridine; e) 4 eq Mtt- $\gamma$-OH, 4 eq PyBOP, 12 eq NMM; f) 4 eq Mtt- $\gamma-$ $\mathrm{OH}, 4$ eq PyBOP, 8 eq NMM; g) 4 eq Mtt- $\gamma-\mathrm{OH}, 4$ eq PyBOP, 8 eq pyridine. 


\section{References}

[i] H. E. Gottlieb, V. Kotlyar, A. Nudelman J. Org. Chem. 1997, 62, 7512-7515.

[ii] E. E. Baird, P. B. Dervan J. Am. Chem. Soc. 1996, 118, 6141-6146.

[iii] M. Minoshima, T. Bando, S. Sasaki, J. Fujimoto, H. Sugiyama Nucleic Acids Res. 2008, 36, 9, 2889-2894.

[iv] J.W. Lown, K. Krowicki J. Org. Chem. 1985, 50, 3774 - 3779.

[v] C. Hotzel, A. Marotto, U. Pindur Eur. J. Med. Chem. 2002, 37, 367 - 378.

[vi] B. König, M. Rödel Synth. Commun. 1999, 29, 943-950.

[vii] C. Hotzel, A. Marotto, U. Pindur Eur. J. Med. Chem. 2003, 38, 2, 189-197.

[viii] B. Heinrich, K. Bouazoune, M. Wojcik, U. Bakowsky, O. Vázquez Org. Biomol. Chem. 2019, 17, 7, 1827-1833.

[ix] A. Viger, P. B. Dervan Bioorg. Med. Chem. 2006, 15, 14, 8539-8549.

[x] P.B. Dervan, E. E. Baird, Method for the synthesis of pyrrole and imidazole carboxamides on a solid support. U.S. Patent 6545162, April 8, 2003.

[xi] E. Nishiwaki, S. Tanaka, H. Lee, M. Shibuya Heterocycles 1988, 27, 1945-1952.

[xii] D. Jaramillo, Q. Liu, J. Aldrich-Wright, Y. Tor J. Org. Chem. 2004, 46, 3492-3497.

[xiii] A. J. Fallows, I. Singh, R. Dondi, P. M. Cullis, G. A. Burley Org. Lett. 2014, 16, 17, 4654-4657.

[xiv] N. R. Wurtz, J. M. Turner, E. E. Baird, P. B. Dervan Org. Lett. 2001, 3, 8, 1201-1203.

[xv] K. Seio, M. Mizuta, T. Terada, M. Sekine J. Org. Chem. 2005, 70, 10311-10322.

[xvi] T. Lescrinier, C. Hendrix, L. Kerremans, J. Rozenski, A. Link, B. Samyn, A. V. Aerschot, E. Lescrinier, R. Eritja, J. V. Beeumen, P. Herdewijn Chem. Eur. J. 1998, 4, 425-433.

[xvii] N. Shiga, S. Takayanagi, R. Muramoto, T. Murakami, R. Qin, Y. Suzuki, K. Shinohara, A. Kenada, T. Nemoto Bioorg. Med. Chem. Lett. 2017, 27, 10, 2197-2200.

[xviii] J. W. Drijfhout, W. Bloemhoff Int. J. Pept. Protein Res. 1991, 37, 1, 27-32.

[xix] Merck KGaA, Novabiochem ${ }^{\circledR}$ Peptide Synthesis; Merck KGaA: Darmstadt, 2012, p 2.32.

[xx] W.J. Fang, T. Yakovleva, J.V. Aldrich Biopolymers 2011, 96, 715-722.

[xxi] G. Kallansrud, B. Ward Anal. Biochem. 1996, 236, 134-138.

[xxii] J. L. Meier, A. S. Yu, I. Korf, D. J. Segal, P. B. Dervan J. Am. Chem. Soc. 2012, 134, 42, 17814-17822. 